\title{
Enantioselective Ruthenium-BINAP-Catalyzed Carbonyl Reductive Coupling of Alkoxyallenes: Convergent Construction of syn-sec,tert-Diols via (Z)- $\sigma$-Allylmetal Intermediates
}

\section{Supporting Information}

\author{
Ming Xiang, Dana E. Pfaffinger, Eliezer Ortiz, \\ Gilmar A. Brito and Michael J. Krische \\ University of Texas at Austin, Department of Chemistry, Austin, TX \\ 78712, USA
}




\section{Table of Contents}

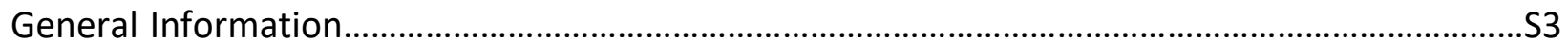

Spectroscopy, Spectrometry, and Data Collection........................................................................S3

Synthesis of $O$-benzhydryl 3-alkoxy-1,2-butadiene (Allene) 1a............................................S4-S7

Synthesis of $O$-benzyl 3-alkoxy-1,2-butadiene (Allene) 1b..................................................S8-S9

Selected Optimization Experiments........................................................................................

Synthesis of Previously Unknown Aldehydes......................................................................S11-S24

Procedures and Spectral Data for The Coupling Products of Benzylic Alcohols and Aldehydes 4a$4 p$. S25-S93

Procedures and Spectral Data for The Coupling Products of Aliphatic Aldehydes $\mathbf{4 q - i s o - 4 j j}$ S94-S165

Total Synthesis of Natural Product (-)-Citreodiol. S166-S170 Isotopic Labeling Studies S171-S174

Spectral Data of s4a for The Coupling Products of allene $\mathbf{1 b}$ and Aldehyde $\mathbf{3 a}$ S175-S177

Single Crystal Diffraction Data for Coupling Products $\mathbf{4 b}, \mathbf{4 j}$ and $\mathbf{4 h h}$ S178-S183

References S184 


\section{General Information}

All reactions were run under an atmosphere of argon, unless otherwise indicated. Resealable pressure tubes $(13 \times 100 \mathrm{~mm}$ ) were purchased from Fischer Scientific (catalog number 14-95935C) and were flame dried followed by cooling in a desiccator or under a stream of argon prior to use. All commercial reagents $\left(\mathrm{RuH}_{2}(\mathrm{CO})\left(\mathrm{PPh}_{3}\right)_{3}, \mathrm{RuHCl}(\mathrm{CO})\left(\mathrm{PPh}_{3}\right)_{3},(R)\right.$-BINAP, (S)-BINAP, (rac)BINAP, Lil) and anhydrous solvents were used as received from vendors (Strem Chemicals, Fischer Scientific, Sigma Aldrich and Combi-Blocks) without further purification. Purification of reaction products was carried out by flash column chromatography using 40-63 $\mu \mathrm{m}$ silica gel. Analytical thin-layer chromatography (TLC) was carried out using $0.25 \mathrm{~mm}$ commercial silica gel plates (Dynamic Absorbents F254). Visualization was accomplished with UV light followed by dipping in Magic stain, $p$-Anisaldehyde (PAA), or $\mathrm{KMnO}_{4}$ stain solution followed by heating.

\section{Spectroscopy, Spectrometry, and Data Collection}

Infrared spectra were recorded on a Perkin-Elmer 1600 spectrometer. High-resolution mass spectra (HRMS) were obtained on a Karatos MS9 and are reported as $\mathrm{m} / \mathrm{z}$ (relative intensity). Accurate masses are reported for the molecular ion $(\mathrm{M}+, \mathrm{M}+\mathrm{H}, \mathrm{M}+\mathrm{Na})$, or a suitable fragment ion. Proton nuclear magnetic resonance $\left({ }^{1} \mathrm{H}\right.$ NMR) spectra were recorded with a Varian INOVA (400, $500 \mathrm{MHz}$ ) spectrometer equipped with a Bruker AVANCE III cryoprobe. Data reported as multiplicity ( $\mathrm{s}=$ singlet, $\mathrm{d}=$ doublet, $\mathrm{t}=$ triplet, $\mathrm{q}=$ quartet, $\mathrm{m}=$ multiplet). Integration and coupling constants were reported in Hertz $(\mathrm{Hz})$. Carbon-13 nuclear magnetic resonance $\left({ }^{13} \mathrm{C}\right.$ NMR) spectra were recorded with a Varian INOVA $(100,125 \mathrm{MHz})$ spectrometer and were routinely run with broadband decoupling. Fluorine-19 nuclear magnetic resonance $\left({ }^{19} \mathrm{~F} \mathrm{NMR}\right)$ spectra were recorded with a Varian INOVA $(390,470 \mathrm{MHz})$ spectrometer. Deuterium nuclear magnetic resonance $\left({ }^{2} \mathrm{H}\right.$ NMR) spectra were recorded in $\mathrm{CHCl}_{3}$ solution with a Varian Gemini 500 (92 $\mathrm{MHz}$ ) spectrometer (relaxation delay 2.00s). 


\section{Synthesis of $O$-benzhydryl 3-alkoxy-1,2-butadiene (1a)}

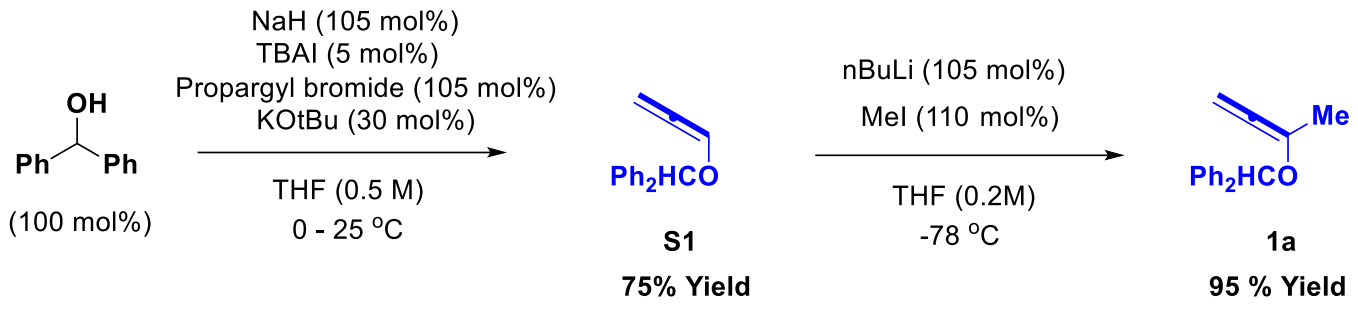

\section{Synthesis of mono-substituted alkoxy-allene S1}

An oven-dried round-bottomed flask equipped with a magnetic stir bar under an argon atmosphere was charged with $\mathrm{NaH}(4.2 \mathrm{~g}, 105 \mathrm{mmol}, 105 \mathrm{~mol} \%, 60 \%$ in mineral oil) and THF (200 $\mathrm{mL})$. Benzhydryl alcohol (18.4 g, $100 \mathrm{mmol}, 100 \mathrm{~mol} \%$ ) was added portionwise at $0{ }^{\circ} \mathrm{C}$ and TBAl (1.85 g, $5 \mathrm{mmol}, 5 \mathrm{~mol} \%)$ was added. The reaction mixture was stirred at $0{ }^{\circ} \mathrm{C}$ for 1 hour, at which point a solution of propargyl bromide (80\% wt in $9.2 \mathrm{M} \mathrm{PhMe}, 11.4 \mathrm{~mL}, 105 \mathrm{mmol}, 105 \mathrm{~mol} \%$ ) was added dropwise. The reaction mixture was allowed to stir for 3 hours at room temperature. After TLC showed almost full conversion, KtOBu (3.36 g, $30 \mathrm{mmol}, 30 \mathrm{mmol} \%)$ was added. The reaction mixture was allowed to stir 2 hours. The reaction mixture was diluted with hexanes (300 $\mathrm{mL}$ ), filtered through a pad of silica gel with the aid of hexanes. The filtrate was concentrated in vacuo and the residue was subjected to flash column chromatography ( $\mathrm{SiO}_{2}$ : neat hexanes) to furnish the mono-substituted alkoxy-allene $\mathbf{S 1}(16.7 \mathrm{~g}, 75.0 \mathrm{mmol})$ as a colorless oil in $75 \%$ Yield.

${ }^{1} \mathrm{H}$ NMR $\left(500 \mathrm{MHz}, \mathrm{CDCl}_{3}\right) \delta: 7.28-7.23(\mathrm{~m}, 8 \mathrm{H}), 7.20-7.15(\mathrm{~m}, 2 \mathrm{H}), 6.71(\mathrm{t}, \mathrm{J}=6.0 \mathrm{~Hz}, 1 \mathrm{H}), 5.68$ $(\mathrm{s}, 1 \mathrm{H}), 5.22(\mathrm{~d}, J=6.0 \mathrm{~Hz}, 2 \mathrm{H})$.

${ }^{13} \mathrm{C}$ NMR $\left(125 \mathrm{MHz}, \mathrm{CDCl}_{3}\right)$ $\delta: 202.2,141.5,128.5,127.7,127.2,120.6,91.0,82.0$.

FTIR (neat): 1954, 1656, 1512, 1178, 1019, $806 \mathrm{~cm}-1$

HRMS $(\mathrm{M}+, m / z)$ for $\mathrm{C}_{16} \mathrm{H}_{14} \mathrm{O}$ : calcd. = 222.1045; found $=222.1045$. 

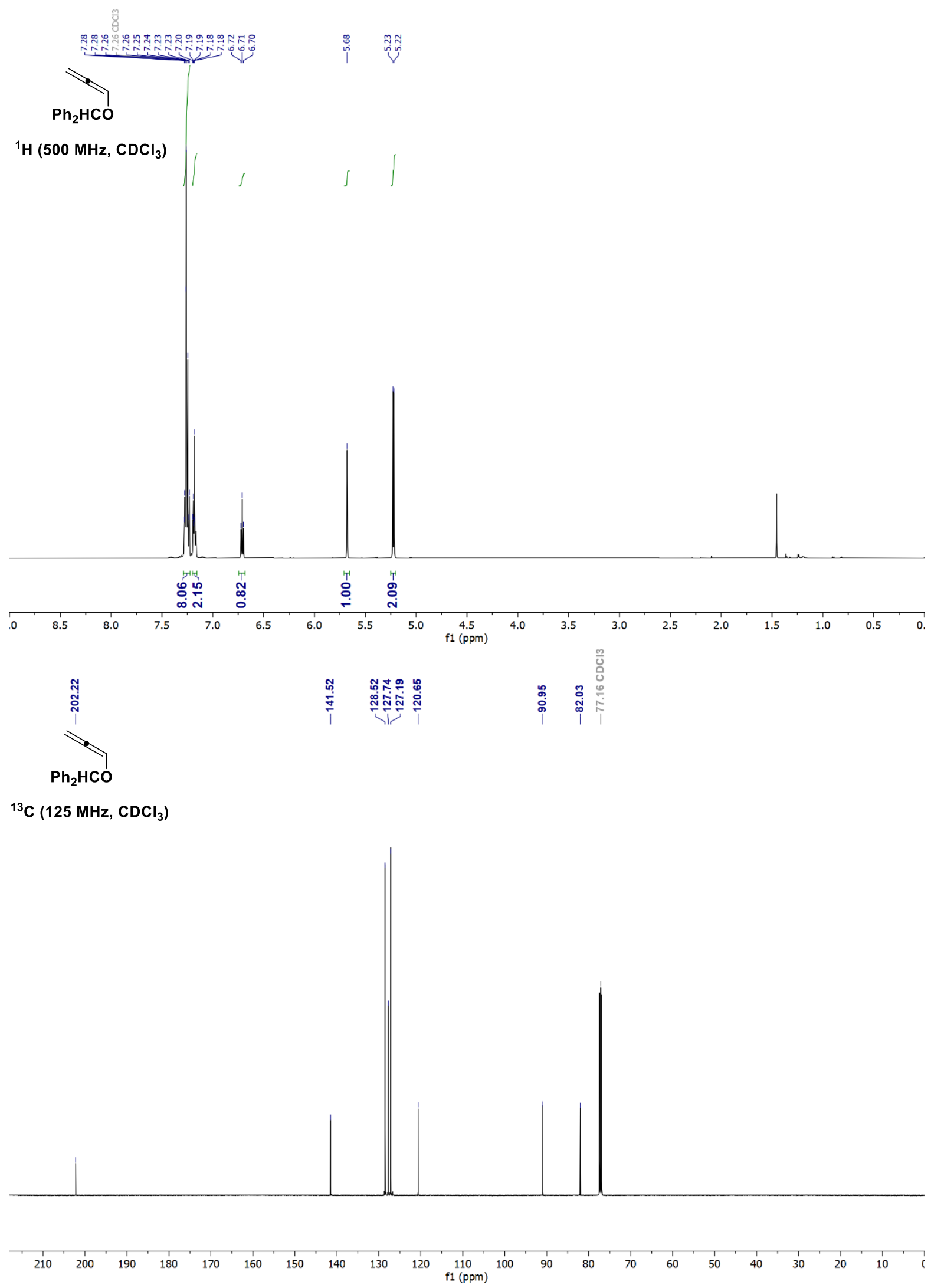

S5 


\section{Synthesis of $O$-benzhydryl 3-alkoxy-1,2-butadiene (1a)}

To an oven-dried round-bottomed flask equipped with a magnetic stir bar under an argon atmosphere was added mono-substituted alkoxy-allene $\mathbf{S 1}(15.0 \mathrm{~g}, 67.5 \mathrm{mmol}, 100 \mathrm{~mol} \%)$ in THF $(340 \mathrm{~mL}, 0.2 \mathrm{M})$. The solution was cooled to $-78{ }^{\circ} \mathrm{C}$, at which point $\mathrm{n}$-BuLi $(28.3 \mathrm{~mL}, 70.8 \mathrm{mmol}$, $105 \mathrm{~mol} \%$ ) was added dropwise. After stirring at $-78^{\circ} \mathrm{C}$ for 3 hours, Mel $(4.6 \mathrm{~mL}, 74.2 \mathrm{mmol}, 110$ mol\%) was added. The reaction was allowed to stir at $-78{ }^{\circ} \mathrm{C}$ for 2 hours, at which point it was diluted with hexanes $(600 \mathrm{~mL})$. The reaction mixture was kept at $-78{ }^{\circ} \mathrm{C}$ for 20 mins and then filtered through neutral alumina (pre-treated with cold hexanes) with the aid of cold hexanes. The solvent was removed in vacuo to afford the title compound (15.2 g, $64.3 \mathrm{mmol}$ ) as a colorless oil in 95\% Yield. 【Cold filtration is important to avoid isomerization of allene to diene. Lil can be washed out with DI-water if the allene is not colorless 】

${ }^{1} \mathbf{H}$ NMR $\left(500 \mathrm{MHz},\left(\mathrm{CD}_{3}\right)_{2} \mathrm{CO}\right) \delta: 7.41-7.36(\mathrm{~m}, 4 \mathrm{H}), 7.32(\mathrm{dd}, J=8.5,6.9 \mathrm{~Hz}, 4 \mathrm{H}), 7.27-7.20$ $(\mathrm{m}, 2 \mathrm{H}), 5.79(\mathrm{~s}, 1 \mathrm{H}), 5.15(\mathrm{q}, J=2.9 \mathrm{~Hz}, 2 \mathrm{H}), 1.93(\mathrm{t}, J=2.9 \mathrm{~Hz}, 3 \mathrm{H})$.

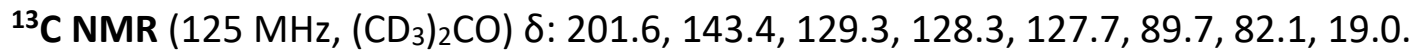

FTIR (neat): 1732, 1494, 1264, 1190, 1026, 893, $737 \mathrm{~cm}-1$

HRMS $(\mathrm{M}+, m / z)$ for $\mathrm{C}_{17} \mathrm{H}_{16} \mathrm{O}$ : calcd. = 236.1201; found $=236.1198$. 

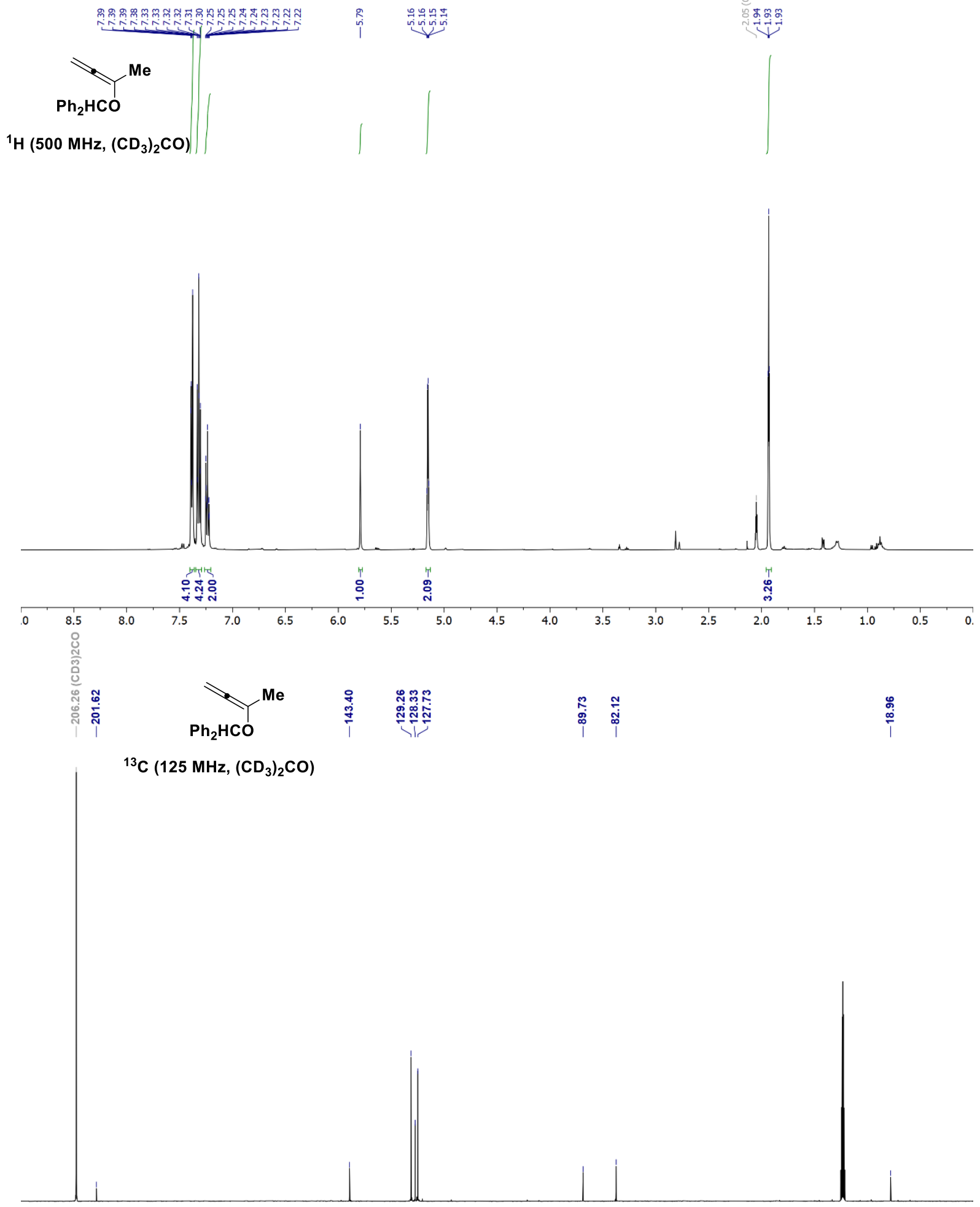

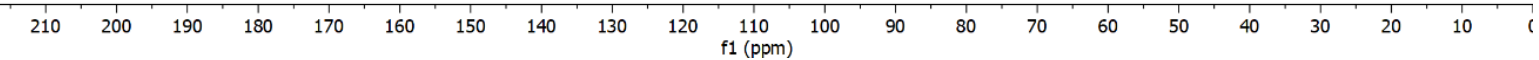




\section{Synthesis of 0 -benzyl 3-alkoxy-1,2-butadiene (1b)}

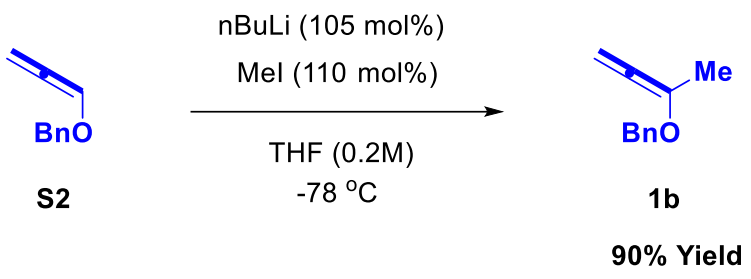

To an oven-dried dry round-bottomed flask equipped with a magnetic stir bar under an argon atmosphere was added mono-substituted alkoxy-allene $\mathbf{S 2} .^{1}$ (4.4 g, $30 \mathrm{mmol}, 100 \mathrm{~mol} \%$ ) in THF $(150 \mathrm{~mL}, 0.2 \mathrm{M})$. The solution was cooled to $-78{ }^{\circ} \mathrm{C}$, at which point $\mathrm{n}$-BuLi $(12.6 \mathrm{~mL}, 31.5 \mathrm{mmol}$, $105 \mathrm{~mol} \%)$ was added dropwise. After stirring at $-78{ }^{\circ} \mathrm{C}$ for 3 hours, Mel $(2.0 \mathrm{~mL}, 33 \mathrm{mmol}, 110$ mol\%) was added. The reaction was allowed to stir at $-78{ }^{\circ} \mathrm{C}$ for 2 hours, at which point it was diluted with hexanes $(300 \mathrm{~mL})$. The reaction mixture was kept at $-78{ }^{\circ} \mathrm{C}$ for 20 mins and then filtered through neutral alumina (pre-treated with cold hexanes) with the aid of cold hexanes. The solvent was removed in vacuo to afford the title compound $(4.3 \mathrm{~g}, 27 \mathrm{mmol})$ as a colorless oil in $90 \%$ Yield. 【Cold filtration is important to avoid isomerization of allene to diene. Lil can be washed out with DI-water if the allene is not colorless 】

${ }^{1} \mathrm{H}$ NMR $\left(500 \mathrm{MHz},\left(\mathrm{CD}_{3}\right)_{2} \mathrm{CO}\right) \delta: 7.45-7.25(\mathrm{~m}, 5 \mathrm{H}), 5.35(\mathrm{q}, J=2.9 \mathrm{~Hz}, 2 \mathrm{H}), 4.58(\mathrm{~s}, 2 \mathrm{H}), 1.89(\mathrm{t}$, $J=2.9 \mathrm{~Hz}, 3 \mathrm{H})$.

${ }^{13} \mathrm{C}$ NMR $\left(125 \mathrm{MHz},\left(\mathrm{CD}_{3}\right){ }_{2} \mathrm{CO}\right) \delta: 200.7,139.0,129.3,128.6,128.6,89.6,71.2,18.7$.

FTIR (neat): 1969, 1454, 1295, 1264, 1197, 1046, $737 \mathrm{~cm}-1$

HRMS $(\mathrm{Ag}+, m / z)$ for $\mathrm{C}_{11} \mathrm{H}_{12} \mathrm{O}$ : calcd. $=266.9934 ;$ found $=266.9931$. 

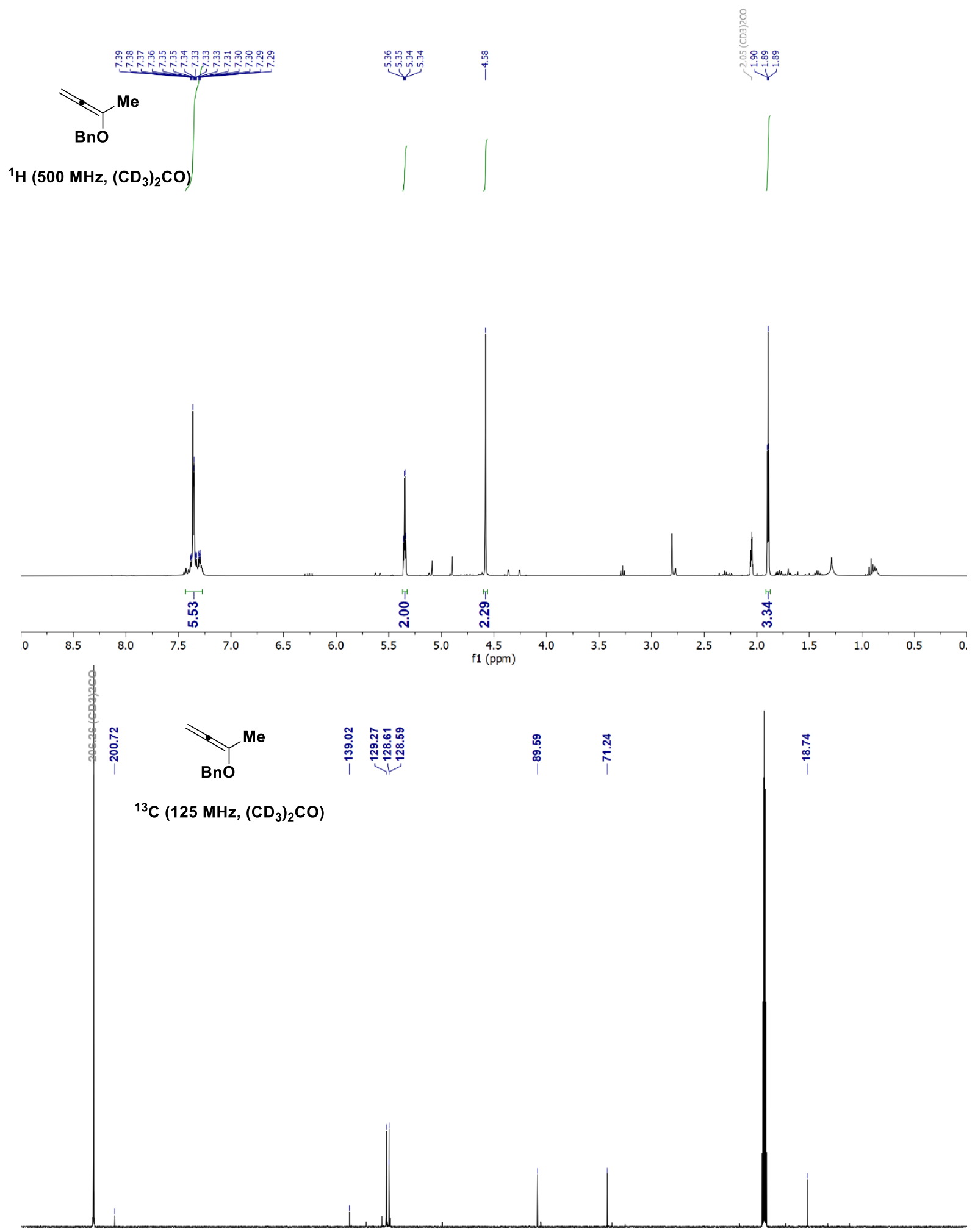

$\begin{array}{llllllllllllllllllllllllllllll}220 & 210 & 200 & 190 & 180 & 170 & 160 & 150 & 140 & 130 & 120 & 110 & 100 & 90 & 80 & 70 & 60 & 50 & 40 & 30 & 20 & 10 & 0 & -10 & -20\end{array}$ 


\section{Selected Optimization Experiments}

\section{For Benzylic System:}

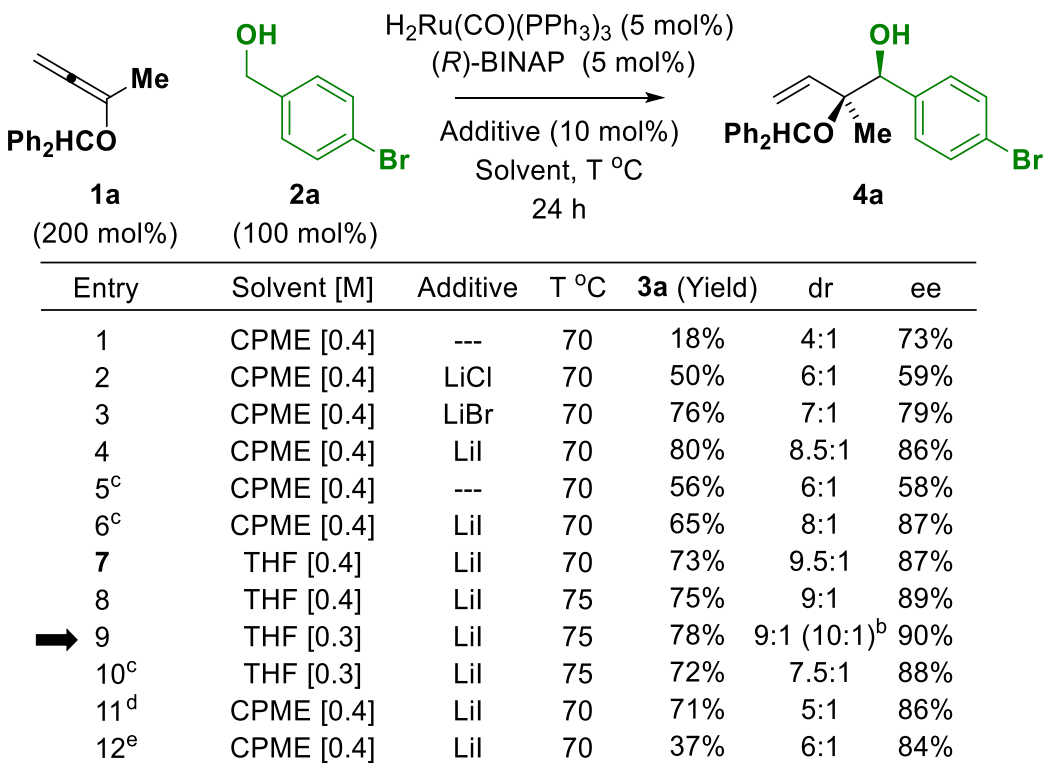

Diastereoselectivities were determined by ${ }^{1} \mathrm{H}$ NMR of crude reaction mixtures. Enantioselectivities were determined by chiral stationary phase HPLC analysis. ${ }^{b}$ Diastereoselectivity after chromatographic purification. ${ }^{\mathrm{C}} \mathrm{HClRu}(\mathrm{CO})\left(\mathrm{PPh}_{3}\right)_{3}$ (5 mol\%). ${ }^{d}(R)$-Segphos as ligand. ${ }^{e}(R)$-MeO-BIPHEP as ligand.

\section{For Aliphatic System:}

\begin{tabular}{|c|c|c|c|c|c|c|}
\hline $\begin{array}{c}\mathrm{Ph}_{2} \mathrm{HCO} \\
1 \mathrm{a} \\
(200 \mathrm{~mol} \%)\end{array}$ & $\underset{3 q}{3 q}$ & $\begin{array}{r}\mathrm{Ru}(\mathrm{CO})(\mathrm{F} \\
(\mathrm{R}) \text {-BIN } \\
\text { Additiv } \\
\text { 2-PrOH } \\
\text { Sol }\end{array}$ & $\begin{array}{l}\left.\mathrm{h}_{3}\right)_{3} \\
\quad(7.5 \\
(15 \mathrm{n} \\
(300 \mathrm{n} \\
\mathrm{nt}, \mathrm{T} \\
4 \mathrm{~h}\end{array}$ & $\begin{array}{l}.5 \mathrm{~mol} \%) \\
\mathrm{mol} \%) \\
\longrightarrow \text { ol\%) } \\
\mathrm{ol} \%) \quad \mathrm{Ph} \\
\mathrm{C}\end{array}$ & ${ }_{2} \mathrm{HCO} \mathrm{Me}$ & \\
\hline Entry & Solvent [M] & Additive & $\mathrm{T}^{\circ} \mathrm{C}$ & 4q (Yield) & $\mathrm{dr}$ & ee \\
\hline 1 & THF [0.4] & Lil & 75 & $45 \%$ & $6: 1$ & $97 \%$ \\
\hline 2 & MTBE [0.4] & Lil & 75 & $54 \%$ & $7: 1$ & $97 \%$ \\
\hline 3 & CPME [0.4] & Lil & 75 & $48 \%$ & $10: 1$ & $97 \%$ \\
\hline 4 & TAME [0.4] & Lil & 75 & $63 \%$ & $6: 1$ & $97 \%$ \\
\hline 5 & toluene $[0.4]$ & Lil & 75 & $50 \%$ & $5: 1$ & $97 \%$ \\
\hline 6 & DIPE [0.4] & Lil & 75 & $72 \%$ & $9: 1(10: 1)^{b}$ & $97 \%$ \\
\hline 7 & DIPE [0.4] & Lil & 80 & $70 \%$ & $8: 1$ & $97 \%$ \\
\hline $8^{c}$ & DIPE [0.4] & Lil & 75 & $20 \%$ & $8: 1$ & $97 \%$ \\
\hline $9^{d}$ & DIPE [0.4] & Lil & 75 & $62 \%$ & $9: 1$ & $97 \%$ \\
\hline
\end{tabular}

Diastereoselectivities were determined by ${ }^{1} \mathrm{H}$ NMR of crude reaction mixtures. Enantioselectivities were determined by chiral stationary phase HPLC analysis. ${ }^{b}$ Diastereoselectivity after chromatographic purification. ${ }^{c}$ Alcohol oxidation level. ${ }^{d} 5$ mol\% catalyst loading. 


\section{Synthesis of Previously Unknown Aldehydes}

\section{General Procedure A For the Synthesis of Aldehydes}

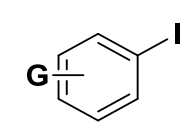

(100 mol\%)

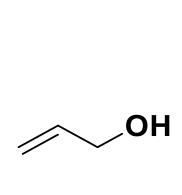

(200 mol\%)

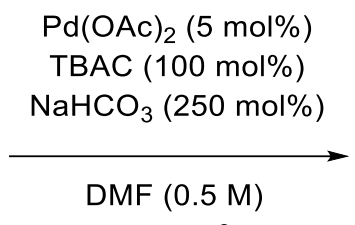

$12 \mathrm{~h}, 50^{\circ} \mathrm{C}$

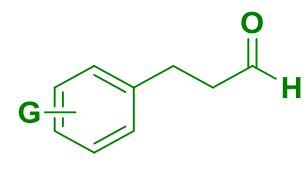

(Ref. 2) To a $100 \mathrm{~mL}$ of round-bottom flask equipped with a magnetic stir bar was added $\mathrm{Pd}(\mathrm{OAc})_{2}$ (5 mol\%), TBAC (100 mol\%), $\mathrm{NaHCO}_{3}(250 \mathrm{~mol} \%)$, Arl (100 mol\%). Then DMF and Allyl alcohol (200 mol\%) were added under Argon balloon, respectively. The flask was heated to $50{ }^{\circ} \mathrm{C}$ (oil bath). After stirring for $12 \mathrm{~h}$ at $50^{\circ} \mathrm{C}$, the reaction mixture was cooled to room temperature, and then sat. $\mathrm{NH}_{4} \mathrm{Cl}$ aq. was poured into the reaction mixture and extracted with $\mathrm{Et}_{2} \mathrm{O}$. The organic layer was washed with water, dried over $\mathrm{Na}_{2} \mathrm{SO}_{4}$, and filtered. The filtrate was concentrated in vacuo and the residue was subjected to silica gel column chromatography to afford aldehydes.

\section{General Procedure B For the Synthesis of Aldehydes}

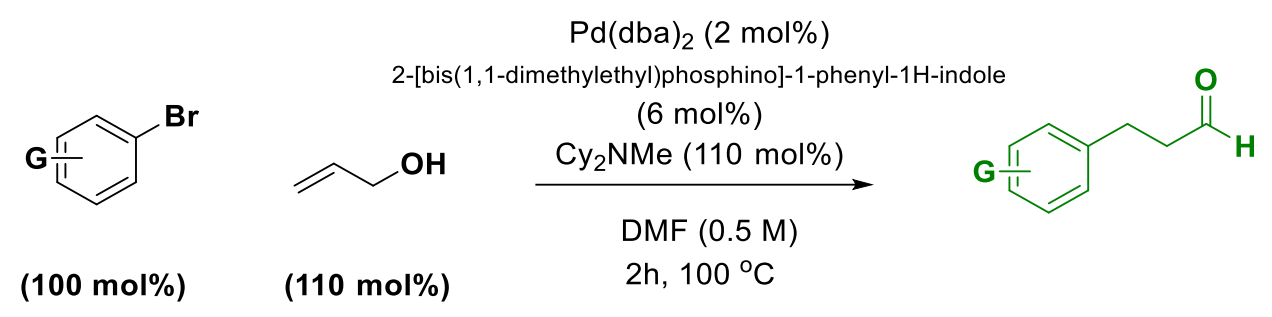

(Ref. 3) To a $100 \mathrm{~mL}$ of round-bottom flask equipped with a magnetic stir bar was added $\mathrm{Pd}(\mathrm{dba})_{2}$ (2 mol\%), 2-[bis(1,1-dimethylethyl)phosphino]-1-phenyl-1H-indole (6 mol\%), $\mathrm{ArBr}$ (100 mol\%). Then DMF and allyl alcohol were added under Argon balloon, respectively. The resulting solution was heated to $100{ }^{\circ} \mathrm{C}$ (oil bath). After stirring for $2 \mathrm{~h}$ at $100^{\circ} \mathrm{C}$, the reaction mixture was cooled to room temperature, and sat. $\mathrm{NH}_{4} \mathrm{Cl}$ aq. was poured into the reaction mixture and extracted with $\mathrm{Et}_{2} \mathrm{O}$. The organic layer was washed with water, dried over $\mathrm{Na}_{2} \mathrm{SO}_{4}$, and filtered. The filtrate was concentrated in vacuo and the residue was subjected to silica gel column chromatography to afford aldehydes. 
tert-butyl (2-fluoro-4-(3-oxopropyl)phenyl)carbamate (3z)

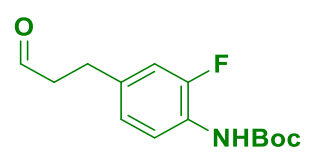

tert-butyl (2-fluoro-4-iodophenyl)carbamate $(1.35 \mathrm{~g}, 4.0 \mathrm{mmol})$ was subjected to general procedure $A$, the reaction was subjected to flash column chromatography $\left(\mathrm{SiO}_{2}, 10: 90\right.$ EtOAc:hexanes) to afford aldehyde $3 z$ ( $0.6 \mathrm{~g}, 2.24 \mathrm{mmol}$ ) as yellow solid in $56 \%$ Yield.

$\operatorname{TLC}\left(\mathrm{SiO}_{2}\right) \mathrm{R}_{\mathrm{f}}=0.25$ (10:90 EtOAc:hexanes)

${ }^{1} \mathbf{H}$ NMR $\left(500 \mathrm{MHz} \mathrm{CDCl}_{3}\right) \delta: 9.80(\mathrm{t}, J=1.2 \mathrm{~Hz}, 1 \mathrm{H}), 7.96(\mathrm{~s}, 1 \mathrm{H}), 6.98-6.82(\mathrm{~m}, 2 \mathrm{H}), 6.64-6.60$ $(\mathrm{m}, 1 \mathrm{H}), 2.89(\mathrm{t}, J=7.4 \mathrm{~Hz}, 2 \mathrm{H}), 2.79-2.70(\mathrm{~m}, 2 \mathrm{H}), 1.52(\mathrm{~s}, 9 \mathrm{H})$.

${ }^{13} \mathrm{C}$ NMR (125 MHz, CDCl 3 ) $\delta: 201.2,152.6,152.2$ (d, $\left.J=242.7 \mathrm{~Hz}\right), 135.8$ (d, $J=6.8 \mathrm{~Hz}$ ), 125.1 (d, $J=10.2 \mathrm{~Hz}$ ), $124.3(\mathrm{~d}, J=3.2 \mathrm{~Hz}), 120.4,114.8(\mathrm{~d}, J=19.1 \mathrm{~Hz}), 81.1,45.2,28.4,27.4$ (d, $J=1.4 \mathrm{~Hz})$.

${ }^{19}$ F NMR (470 MHz, $\left.\mathrm{CDCl}_{3}\right) \delta:-132.3$.

HRMS $(\mathrm{Na}+, m / z)$ for $\mathrm{C}_{14} \mathrm{H}_{18} \mathrm{FNO}_{3}$ : calcd. $=290.1163 ;$ found $=290.1165$. 

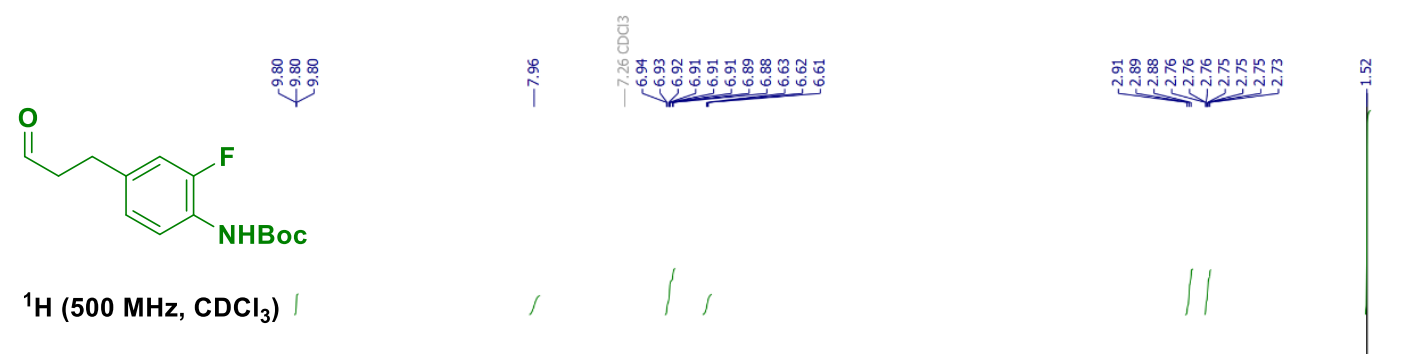

${ }^{1} \mathrm{H}\left(500 \mathrm{MHz}, \mathrm{CDCl}_{3}\right)$
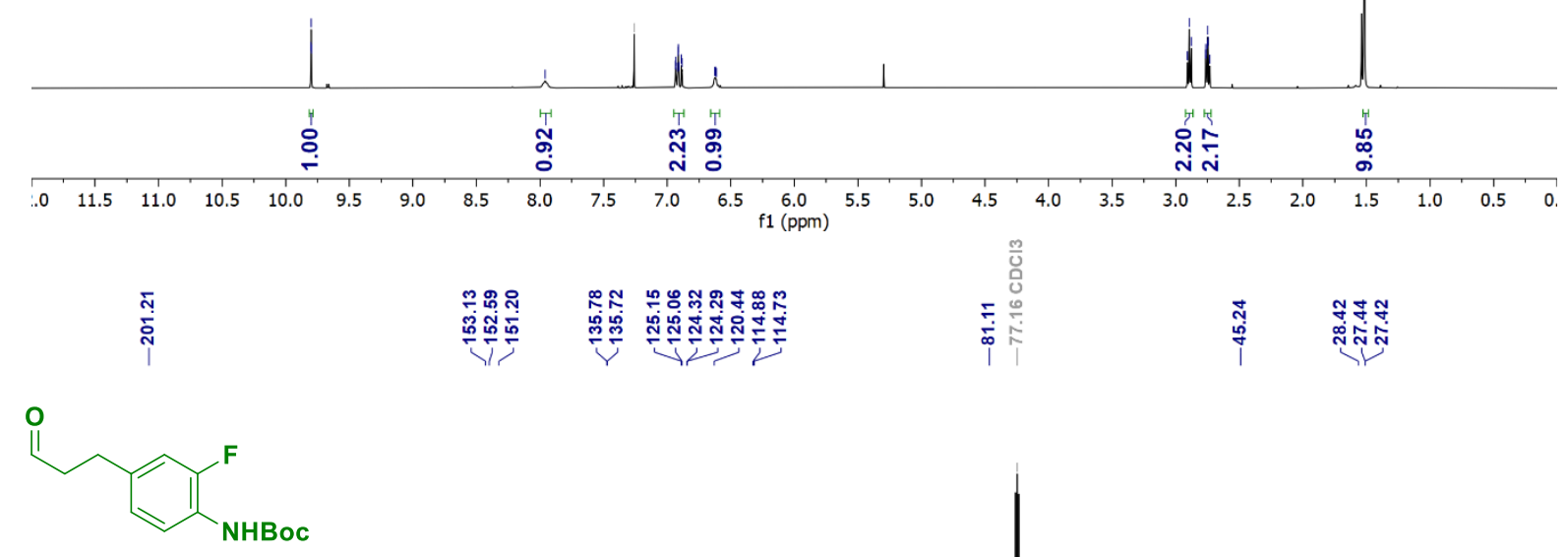

${ }^{13} \mathrm{C}\left(125 \mathrm{MHz}, \mathrm{CDCl}_{3}\right)$
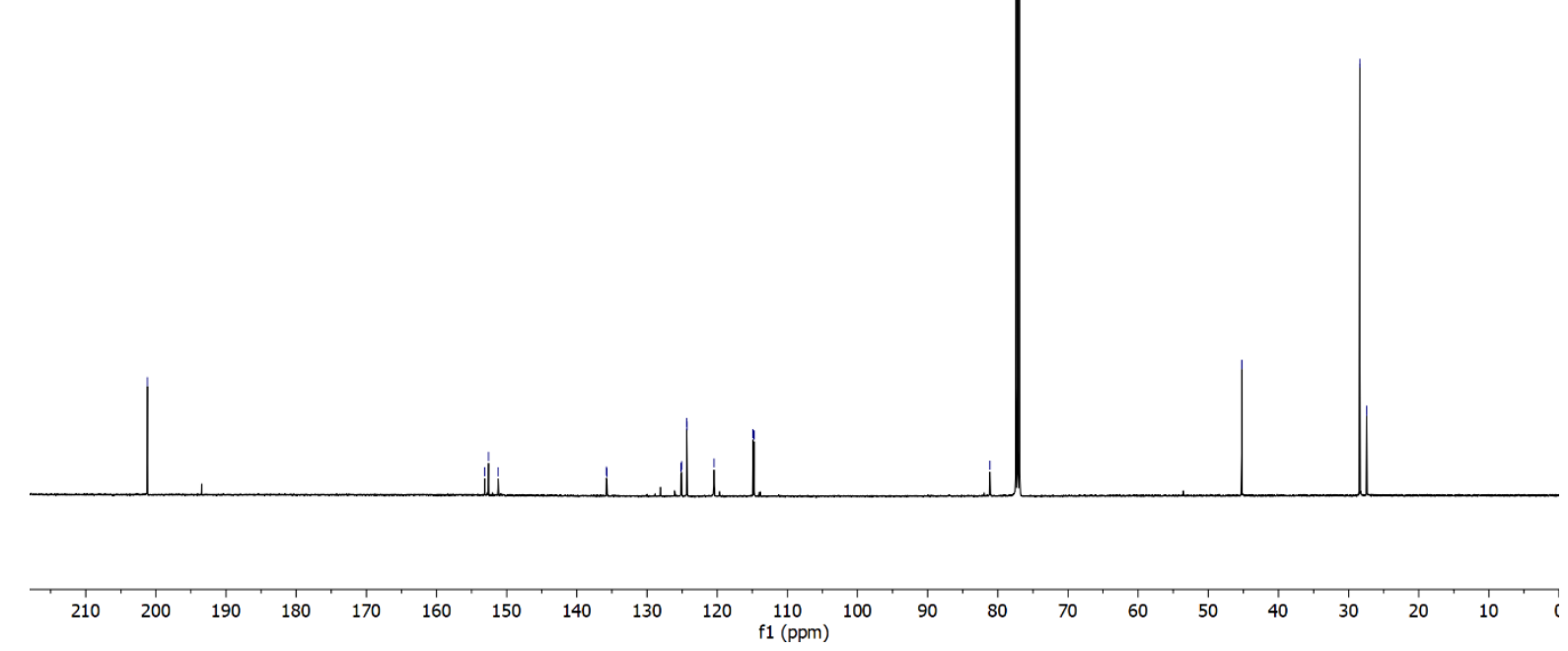
O<smiles>C=CCCc1ccc(Nc2ccccc2)c(F)c1</smiles>

${ }^{19} \mathrm{~F}\left(470 \mathrm{MHz}, \mathrm{CDCl}_{3}\right)$ 
methyl 4-((3-(3-oxopropyl)-1H-indol-1-yl)sulfonyl)benzoate (3aa)

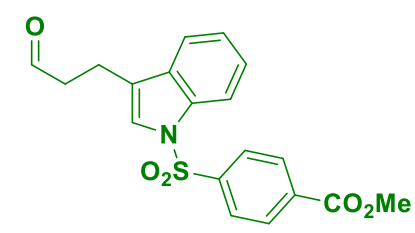

methyl 4-((3-iodo-1H-indol-1-yl)sulfonyl)benzoate (1.51 g, $3.4 \mathrm{mmol})$ was subjected to general procedure $A$, the reaction was subjected to flash column chromatography $\left(\mathrm{SiO}_{2}, 20: 80\right.$ EtOAc:hexanes) to afford aldehyde 3aa $(0.65 \mathrm{~g}, 1.75 \mathrm{mmol})$ as brown solid in $51 \%$ Yield.

$\operatorname{TLC}\left(\mathrm{SiO}_{2}\right) \mathrm{R}_{\mathrm{f}}=0.25$ (20:80 EtOAc:hexanes)

${ }^{1} \mathbf{H}$ NMR $\left(500 \mathrm{MHz}, \mathrm{CDCl}_{3}\right) \delta: 9.77(\mathrm{t}, J=1.3 \mathrm{~Hz}, 1 \mathrm{H}), 8.00(\mathrm{~d}, J=8.5 \mathrm{~Hz}, 2 \mathrm{H}), 7.91(\mathrm{~d}, J=8.3 \mathrm{~Hz}$, $1 \mathrm{H}), 7.83(\mathrm{~d}, J=8.6 \mathrm{~Hz}, 2 \mathrm{H}), 7.41(\mathrm{~d}, J=7.8 \mathrm{~Hz}, 1 \mathrm{H}), 7.31-7.26(\mathrm{~m}, 1 \mathrm{H}), 7.22-7.17(\mathrm{~m}, 2 \mathrm{H}), 3.83$ $(\mathrm{s}, 3 \mathrm{H}), 2.94(\mathrm{t}, J=7.0 \mathrm{~Hz}, 2 \mathrm{H}), 2.78(\mathrm{t}, J=7.1 \mathrm{~Hz}, 2 \mathrm{H})$.

${ }^{13} \mathrm{C}$ NMR $\left(125 \mathrm{MHz}, \mathrm{CDCl}_{3}\right) \delta: 200.9,165.4,141.8,135.4,134.9,130.8,130.5,126.9,125.4,123.8$, $123.0,122.5,119.5,114.0,52.8,42.9,17.5$.

HRMS $(\mathrm{Na}+, \mathrm{m} / z)$ for $\mathrm{C}_{19} \mathrm{H}_{17} \mathrm{NO}_{5} \mathrm{~S}$ : calcd. $=394.0720$; found $=394.0719$. 

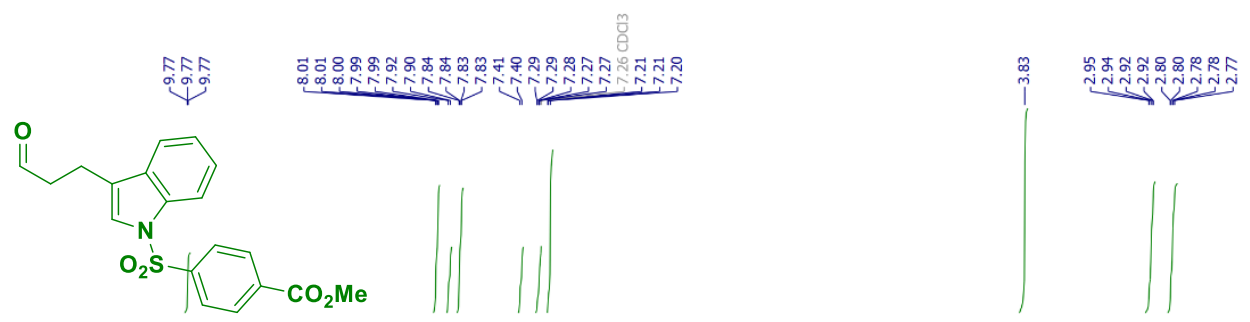

${ }^{1} \mathrm{H}\left(500 \mathrm{MHz}, \mathrm{CDCl}_{3}\right)$
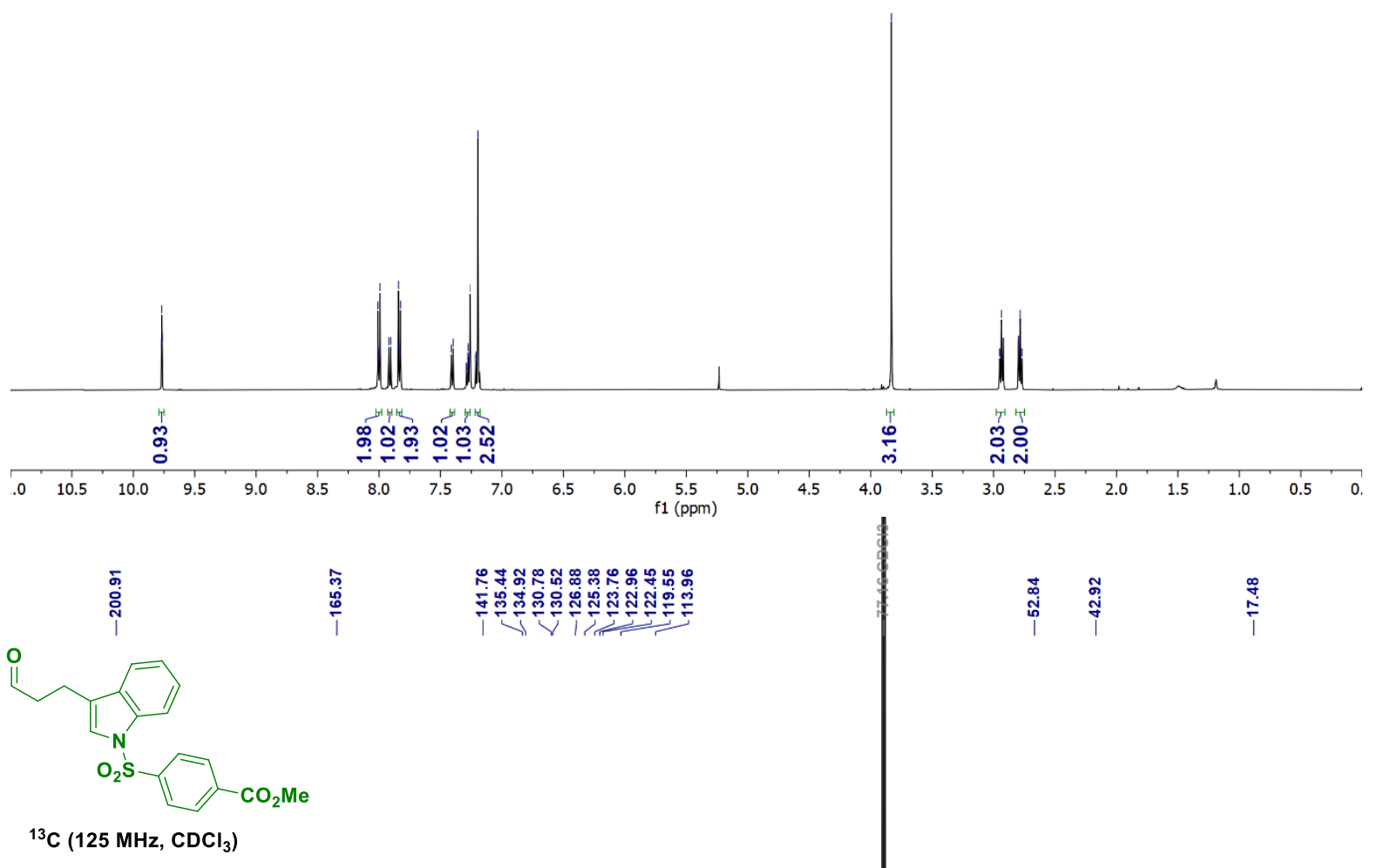

${ }^{13} \mathrm{C}\left(125 \mathrm{MHz}, \mathrm{CDCl}_{3}\right)$

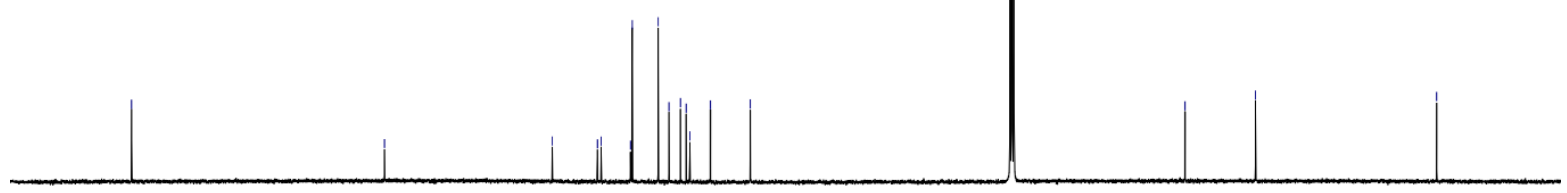

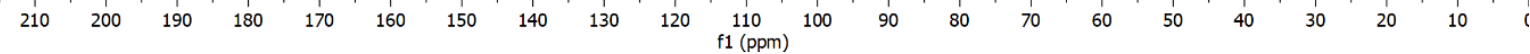

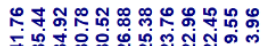

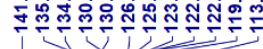

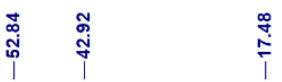




\section{3-(2,2-difluorobenzo[d][1,3]dioxol-5-yl)propanal (3bb)}

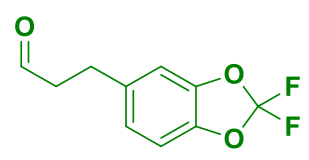

5-bromo-2,2-difluorobenzo[d][1,3]dioxole (1.2 g, $5 \mathrm{mmol}$ ) was subjected to general procedure $\mathrm{B}$, the reaction was subjected to flash column chromatography ( $\mathrm{SiO}_{2}$, 5:95 EtOAc:hexanes) to afford aldehyde $3 \mathrm{bb}$ ( $728 \mathrm{mg}, 3.4 \mathrm{mmol}$ ) as colorless oil in $68 \%$ yield.

$\operatorname{TLC}\left(\mathrm{SiO}_{2}\right) \mathrm{R}_{\mathrm{f}}=0.25$ (5:95 EtOAc:hexanes)

${ }^{1} \mathbf{H}$ NMR $\left(500 \mathrm{MHz}, \mathrm{CDCl}_{3}\right) \delta: 9.81(\mathrm{~s}, 1 \mathrm{H}), 6.99-6.86(\mathrm{~m}, 3 \mathrm{H}), 2.95(\mathrm{t}, J=7.4 \mathrm{~Hz}, 2 \mathrm{H}), 2.78(\mathrm{t}, J=$ $7.4 \mathrm{~Hz}, 2 \mathrm{H})$.

${ }^{13} \mathrm{C}$ NMR $\left(125 \mathrm{MHz}, \mathrm{CDCl}_{3}\right)$ $\delta: 200.8,144.1,142.4,136.7,131.77$ (t, J = 254.8 Hz), 123.4, 109.8, $109.5,45.5,28.0$.

${ }^{19}$ F NMR (470 MHz, $\mathrm{CDCl}_{3}$ ) $\delta:-39.3$.

HRMS $(\mathrm{Na}+, m / z)$ for $\mathrm{C}_{10} \mathrm{H}_{8} \mathrm{~F}_{2} \mathrm{O}_{3}$ : calcd. $=214.0442 ;$ found $=214.0442$. 


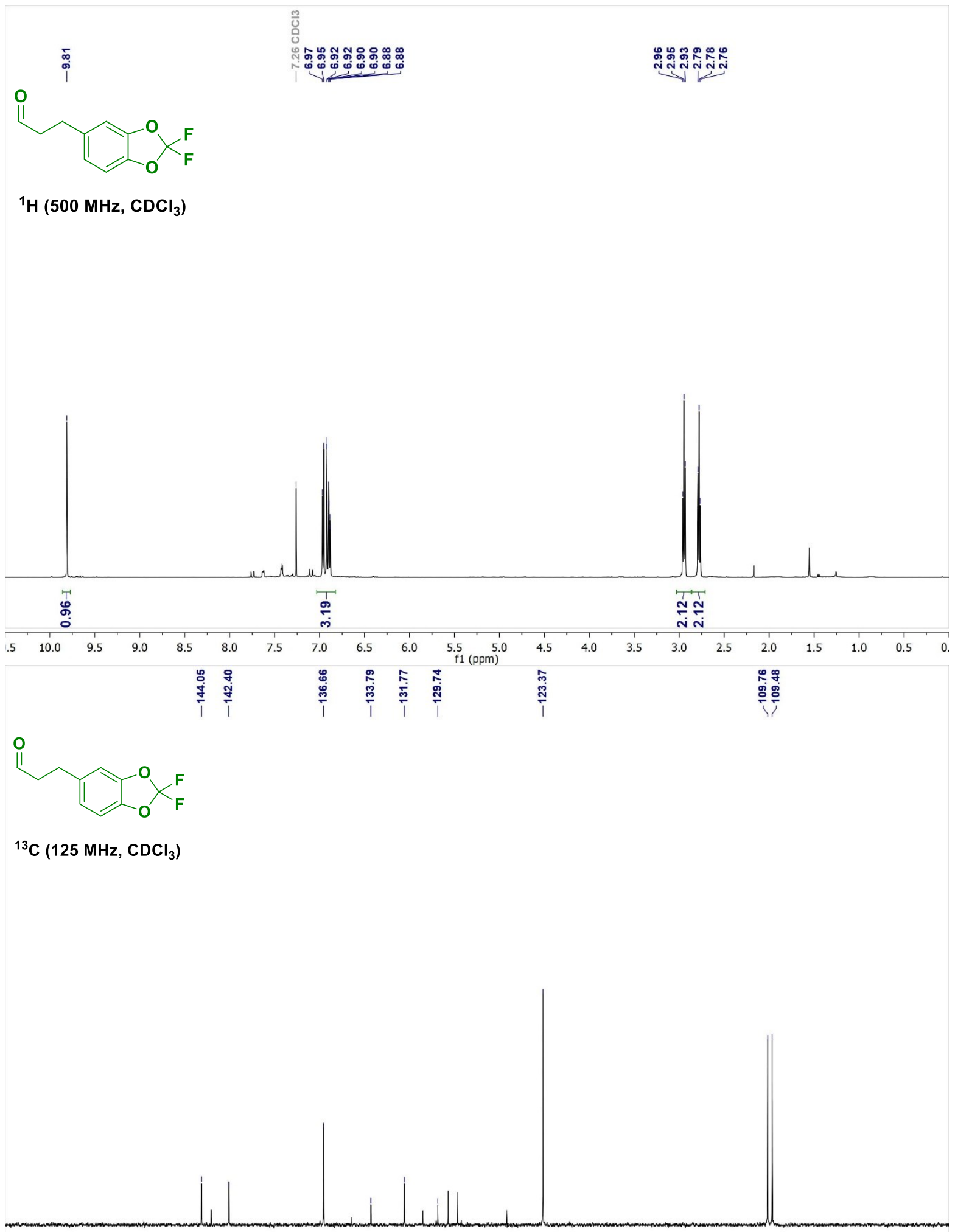

$\begin{array}{llllllllllllllllllllllllllllllllll}154 & 152 & 150 & 148 & 146 & 144 & 142 & 140 & 138 & 136 & 134 & 132 & 130 & 128 & 126 & 124 & 122 & 120 & 118 & 116 & 114 & 112 & 110 & 108 & 106 & 104 & 102 & 100\end{array}$ 


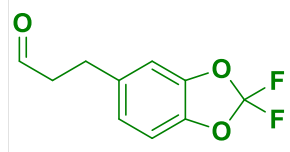

${ }^{19} \mathrm{~F}\left(470 \mathrm{MHz}, \mathrm{CDCl}_{3}\right)$

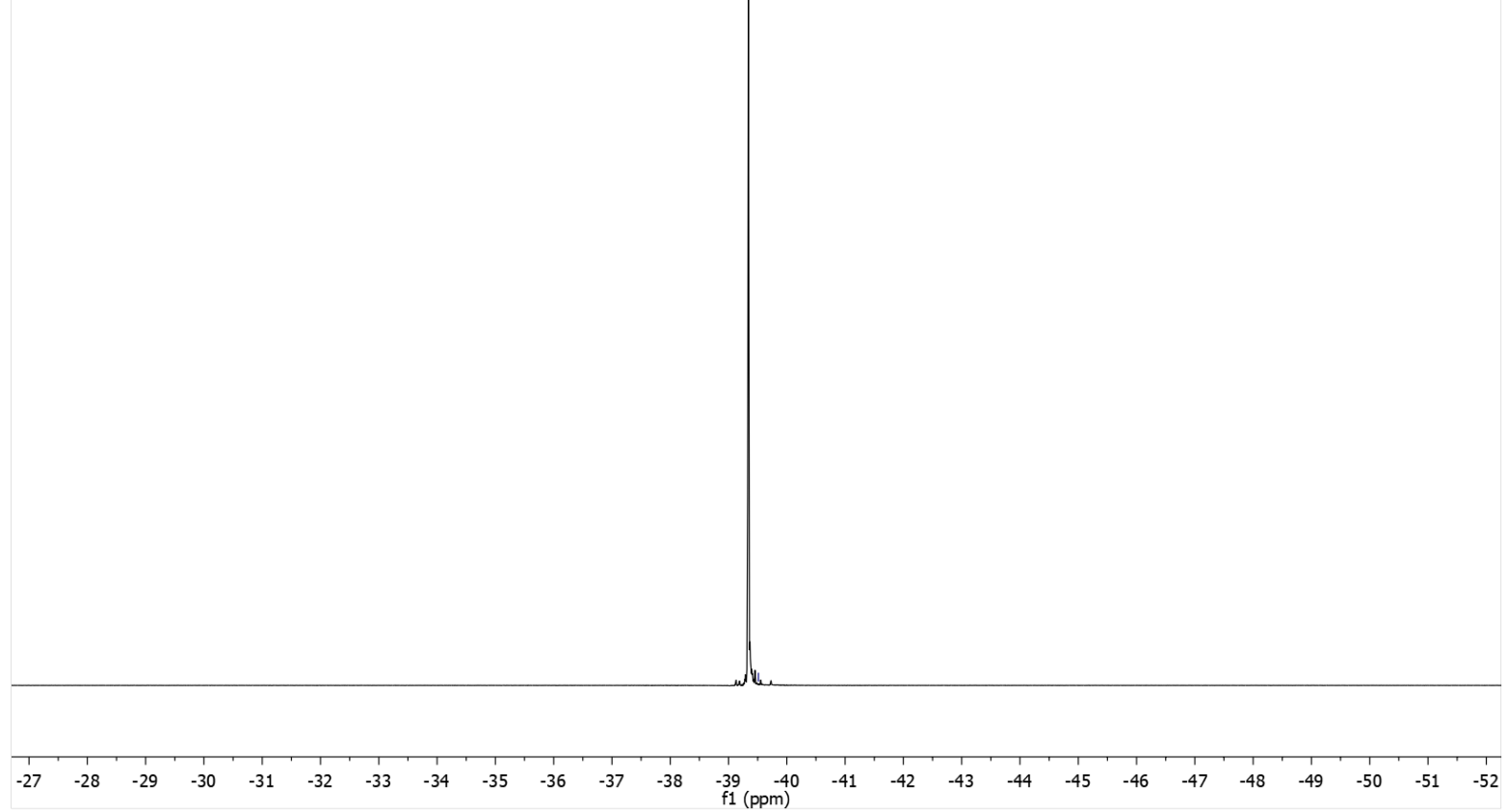


tert-butyl 4-(5-(3-oxopropyl)pyrimidin-2-yl)piperazine-1-carboxylate (3dd)

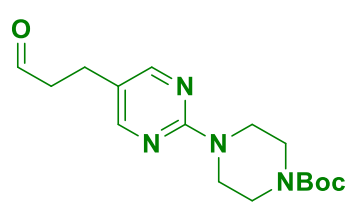

tert-butyl 4-(5-bromopyrimidin-2-yl)piperazine-1-carboxylate $(3.4 \mathrm{~g}, 10 \mathrm{mmol})$ was subjected to general procedure $\mathrm{B}$, the reaction was subjected to flash column chromatography $\left(\mathrm{SiO}_{2}, 30: 70\right.$ EtOAc:hexanes) to afford aldehyde $3 \mathrm{dd}(1.5 \mathrm{~g}, 4.68 \mathrm{mmol}$ ) as brown solid in $47 \%$ Yield.

$\operatorname{TLC}\left(\mathrm{SiO}_{2}\right) \mathrm{Rf}_{\mathrm{f}}=0.3$ (40:60 EtOAc:hexanes)

${ }^{1} \mathrm{H}$ NMR $\left(500 \mathrm{MHz}, \mathrm{CDCl}_{3}\right) \delta: 9.80(\mathrm{~d}, J=1.1 \mathrm{~Hz}, 1 \mathrm{H}), 8.19(\mathrm{~s}, 2 \mathrm{H}), 3.76(\mathrm{dd}, J=6.4,4.2 \mathrm{~Hz}, 4 \mathrm{H})$, $3.54-3.41(\mathrm{~m}, 4 \mathrm{H}), 2.81-2.69(\mathrm{~m}, 4 \mathrm{H}), 1.48(\mathrm{~s}, 9 \mathrm{H})$.

${ }^{13} \mathrm{C} \mathrm{NMR}\left(125 \mathrm{MHz}, \mathrm{CDCl}_{3}\right) \delta: 200.7,161.0,157.7,155.0,121.6,80.1,45.1,43.9,28.6,22.0$.

HRMS $(\mathrm{Na}+, m / z)$ for $\mathrm{C}_{16} \mathrm{H}_{24} \mathrm{~N}_{4} \mathrm{O}_{3}$ : calcd. $=343.1741 ;$ found $=343.1743$. 


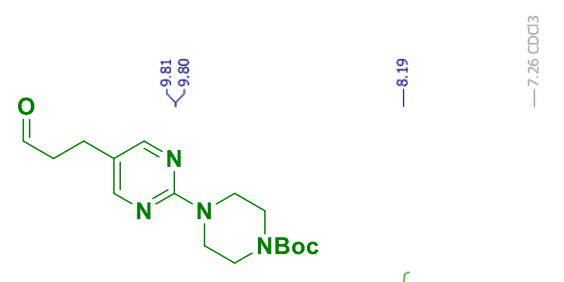

${ }^{1} \mathrm{H}\left(500 \mathrm{MHz}, \mathrm{CDCl}_{3}\right)$
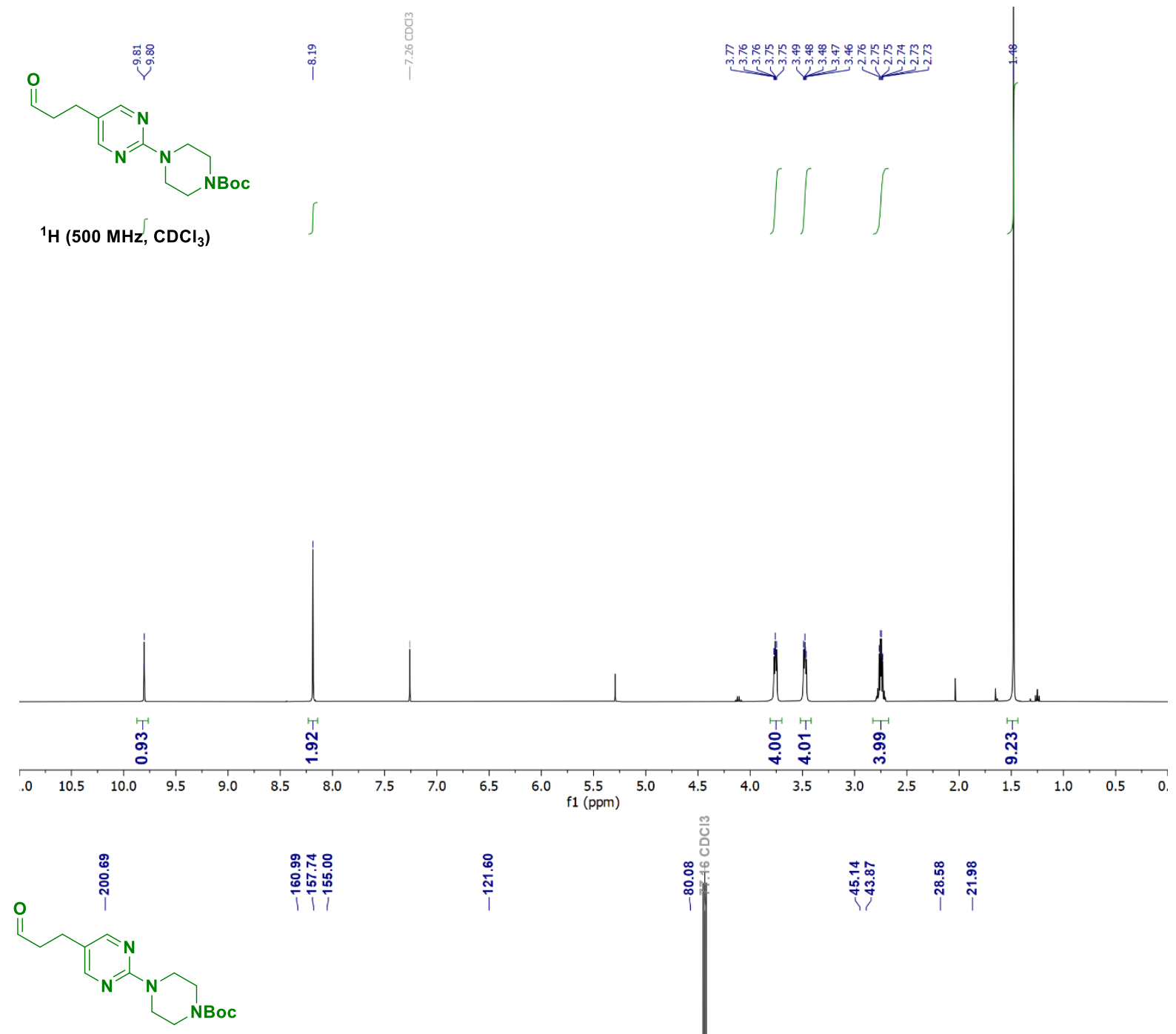

${ }^{13} \mathrm{C}\left(125 \mathrm{MHz}, \mathrm{CDCl}_{3}\right)$
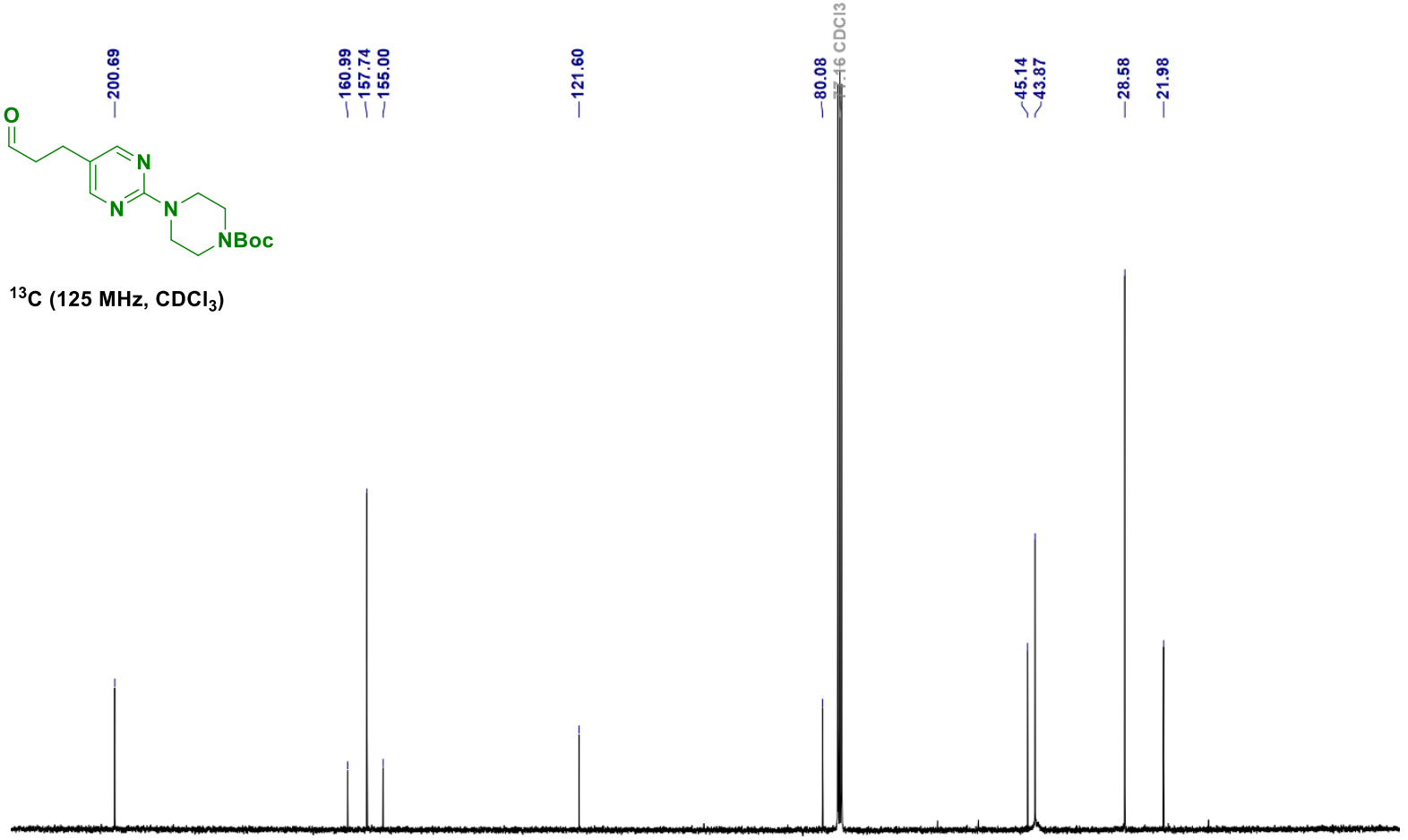

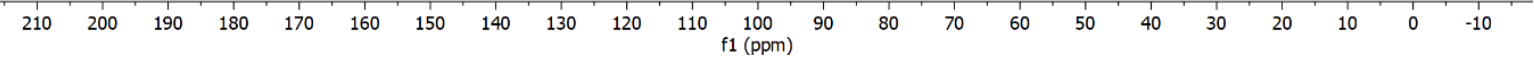




\section{3-(3-(5-(4-fluorophenyl)thiophene-2-carbonyl)-4-methylphenyl)propanal (3ee)}

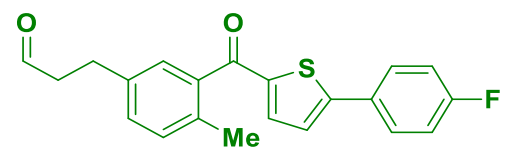

(5-(4-fluorophenyl)thiophen-2-yl)(5-iodo-2-methylphenyl)methanone $(4.2 \mathrm{~g}, 10 \mathrm{mmol})$ was subjected to general procedure $A$, the reaction was subjected to flash column chromatography $\left(\mathrm{SiO}_{2}, 20: 80\right.$ EtOAc:hexanes) to afford aldehyde $3 \mathrm{ee}(3 \mathrm{~g}, 8.5 \mathrm{mmol})$ as brown solid in $85 \%$ yield.

$\operatorname{TLC}\left(\mathrm{SiO}_{2}\right) \mathrm{R}_{\mathrm{f}}=0.2(20: 80$ EtOAc:hexanes)

${ }^{1} \mathrm{H}$ NMR $\left(500 \mathrm{MHz}, \mathrm{CDCl}_{3}\right) \delta: 9.79(\mathrm{t}, J=1.3 \mathrm{~Hz}, 1 \mathrm{H}), 7.65-7.58(\mathrm{~m}, 2 \mathrm{H}), 7.33(\mathrm{~d}, J=4.0 \mathrm{~Hz}, 1 \mathrm{H})$, $7.26(\mathrm{~d}, J=1.6 \mathrm{~Hz}, 1 \mathrm{H}), 7.22(\mathrm{t}, J=3.7 \mathrm{~Hz}, 1 \mathrm{H}), 7.19(\mathrm{~d}, J=1.7 \mathrm{~Hz}, 2 \mathrm{H}), 7.12-7.05(\mathrm{~m}, 2 \mathrm{H}), 2.94(\mathrm{t}$, $J=7.4 \mathrm{~Hz}, 2 \mathrm{H}), 2.76(\mathrm{tt}, J=7.4,0.9 \mathrm{~Hz}, 2 \mathrm{H}), 2.32(\mathrm{~s}, 3 \mathrm{H})$.

${ }^{13} \mathrm{C}$ NMR $\left(125 \mathrm{MHz}, \mathrm{CDCl}_{3}\right) \delta: 201.2,190.2,163.4(\mathrm{~d}, J=249.9 \mathrm{~Hz}), 152.8,143.5,138.7,137.5$, 136.6, 134.5, 131.5, 130.4, 129.7 (d, $J=3.5 \mathrm{~Hz}), 128.3$ (d, $J=8.2 \mathrm{~Hz}), 127.9,124.1,116.4(\mathrm{~d}, J=$ $22.0 \mathrm{~Hz}), 45.2,27.6,19.4$.

${ }^{19}$ F NMR (470 MHz, $\left.\mathrm{CDCl}_{3}\right) \delta:-111.5--111.7(\mathrm{~m})$

HRMS $(\mathrm{Na}+, m / z)$ for $\mathrm{C}_{16} \mathrm{H}_{24} \mathrm{~N}_{4} \mathrm{O}_{3}$ : calcd. $=343.1741$; found $=343.1743$. 

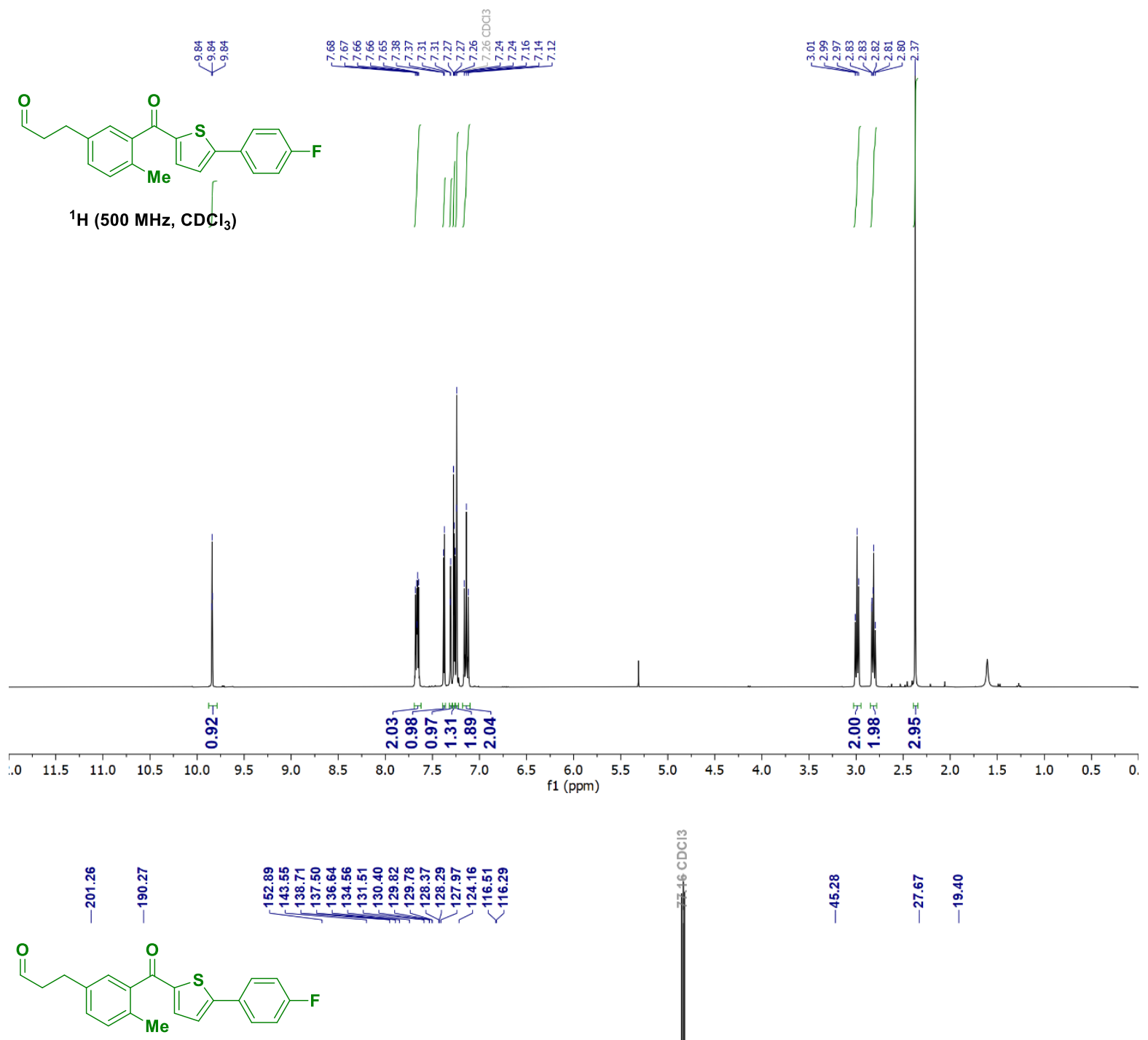

${ }^{13} \mathrm{C}\left(125 \mathrm{MHz}, \mathrm{CDCl}_{3}\right)$

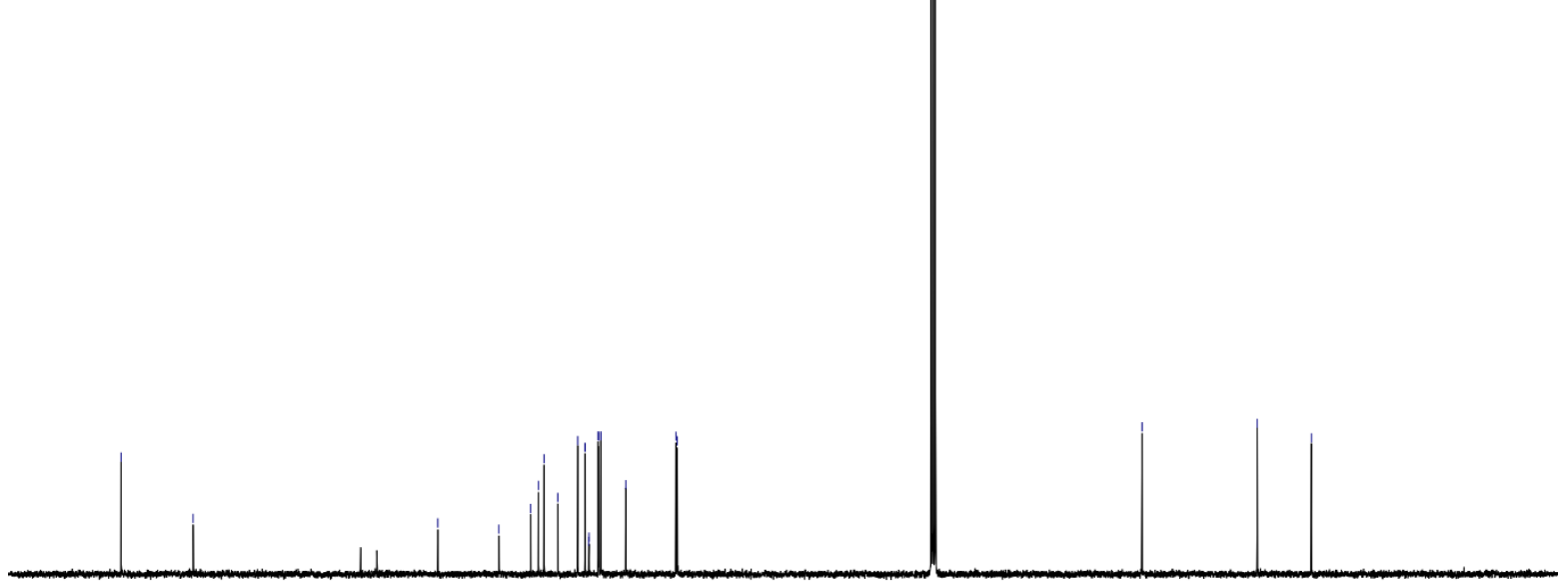

$\begin{array}{lllllllllllllllllllllllll}210 & 200 & 190 & 180 & 170 & 160 & 150 & 140 & 130 & 120 & 110 & 100 & 90 & 80 & 70 & 60 & 50 & 40 & 30 & 20 & 10 & 0 & -10\end{array}$ 


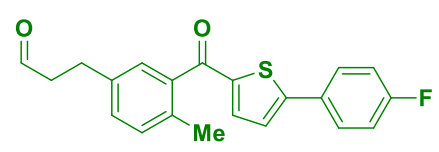

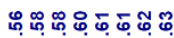

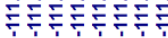

${ }^{19} \mathrm{~F}\left(470 \mathrm{MHz}, \mathrm{CDCl}_{3}\right)$

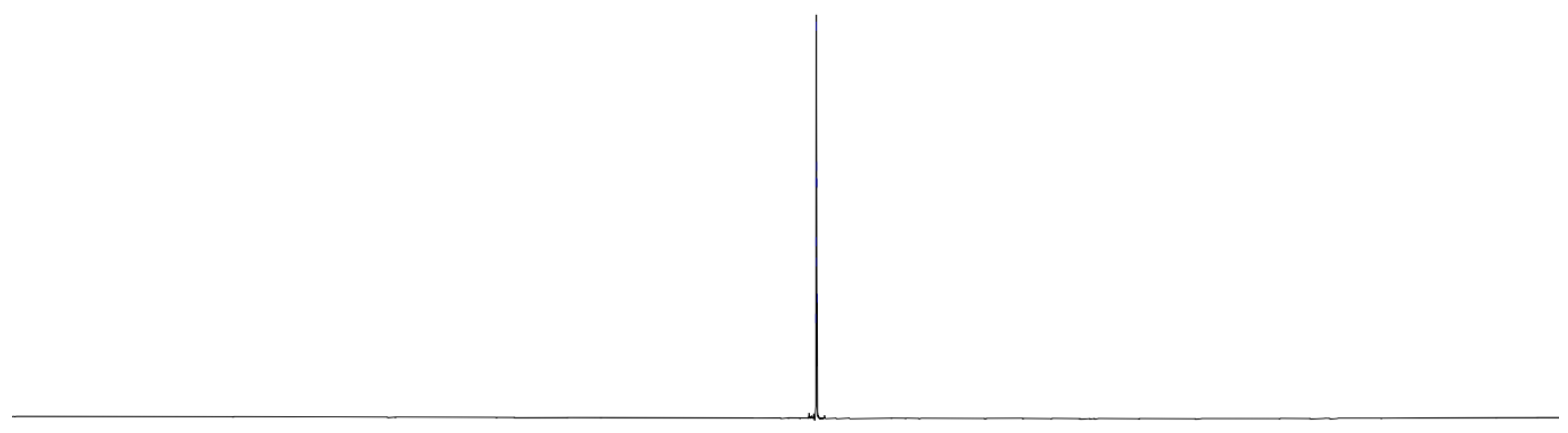

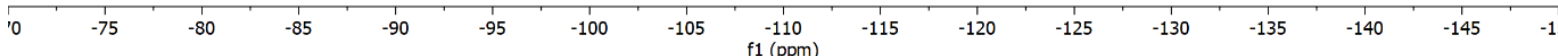


Procedures and Spectral Data for the Coupling Products of Allene and Benzylic Alcohols and Aldehydes 4a-4p

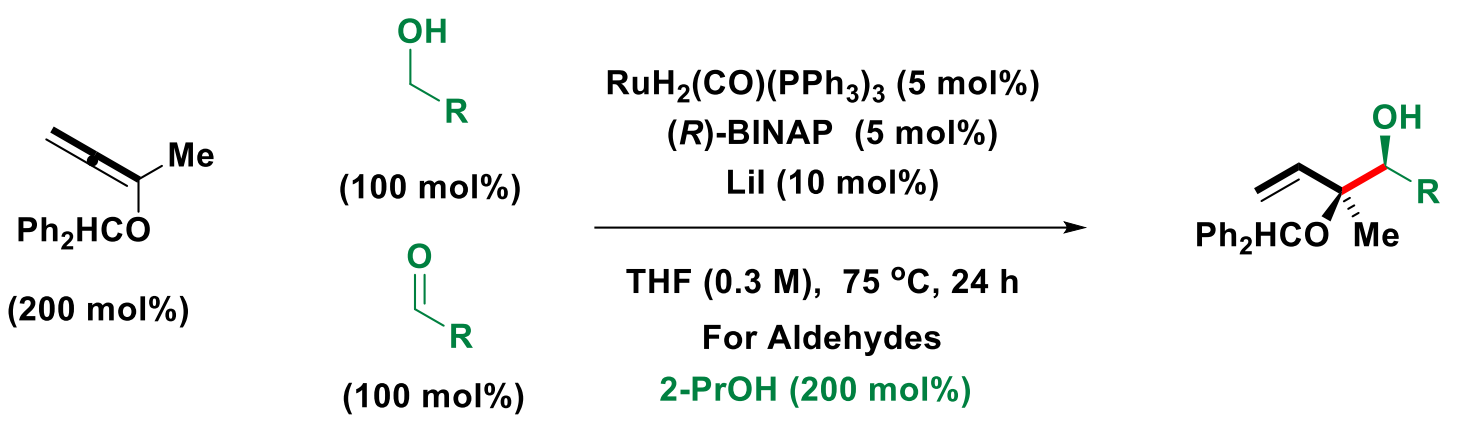

\section{General Procedure for Benzylic alcohols and aldehydes}

At alcohol oxidation level: To a dried pressure tube equipped with a magnetic stir bar under an argon atmosphere charged with Allene $(95.0 \mathrm{mg}, 0.4 \mathrm{mmol}, 200 \mathrm{~mol} \%)$, alcohol $2 \mathrm{a}-2 \mathrm{p}(0.2$ mmol), $\operatorname{RuH}_{\mathbf{2}}(\mathbf{C O})\left(\mathbf{P P h}_{3}\right)_{3}(9.2 \mathrm{mg}, 0.01 \mathrm{mmol}, 5 \mathrm{~mol} \%),(\boldsymbol{R})$-BINAP (6.3 mg, $\left.0.01 \mathrm{mmol}, 5 \mathrm{~mol} \%\right)$, and Lil $(2.7 \mathrm{mg}, 0.02 \mathrm{mmol}, 10 \mathrm{~mol} \%)$ was added THF $(0.66 \mathrm{~mL}, 0.3 \mathrm{M})$. The tube was sealed with a PTFE lined cap and the reaction mixture was allowed to stir for 24 hours at $75^{\circ} \mathrm{C}$. After reaching ambient temperature, the solvent was removed in vacuo and the residue was subjected to flash column chromatography $\left(\mathrm{SiO}_{2}\right)$ under the noted conditions to furnish the products $4 \mathrm{a}-4 \mathrm{p}$. The $\mathrm{dr}$ values were determined by ${ }^{1} \mathrm{H}$ NMR after purification and the enantiomeric excesses were determined by HPLC using chiral stationary phase columns.

At aldehyde oxidation level: To a dried pressure tube equipped with a magnetic stir bar under an argon atmosphere charged with Allene $(95.0 \mathrm{mg}, 0.4 \mathrm{mmol}, 200 \mathrm{~mol} \%)$, aldehyde 3a-3p (0.2 mmol), $\operatorname{RuH}_{2}(\mathbf{C O})\left(\mathbf{P P h}_{3}\right)_{3}$ (9.2 mg, $\left.0.01 \mathrm{mmol}, 5 \mathrm{~mol} \%\right),(\boldsymbol{R})$-BINAP (6.3 mg, $0.01 \mathrm{mmol}, 5 \mathrm{~mol} \%$ ), Lil (2.7 mg, $0.02 \mathrm{mmol}, 10 \mathrm{~mol} \%$ ), and IPA (30 uL, $0.4 \mathrm{mmol}, 200 \mathrm{~mol} \%$ ) was added THF (0.66 mL, $0.3 \mathrm{M}$ ). The tube was sealed with a PTFE lined cap and the reaction mixture was allowed to stir for 24 hours at $75^{\circ} \mathrm{C}$. After reaching ambient temperature, the solvent was removed in vacuo and the residue was subjected to flash column chromatography $\left(\mathrm{SiO}_{2}\right)$ under the noted conditions to furnish the products $4 a-4 p$. The $d r$ values were determined by ${ }^{1} \mathrm{H} N M R$ after purification and the enantiomeric excesses were determined by HPLC using chiral stationary phase columns.

Racemic reactions were performed using (rac)-BINAP as the ligand either at the alcohol or the aldehyde oxidation level under identical conditions. 


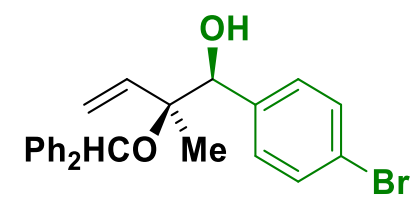

Alcohol oxidation level: Alcohol 2a $(37.4 \mathrm{mg}, 0.2 \mathrm{mmol})$ was subjected to standard reaction conditions $\left(75^{\circ} \mathrm{C}, 24 \mathrm{~h}\right)$. Upon flash column chromatography ( $\mathrm{SiO}_{2}, 5: 95 \mathrm{EtOAc}$ :hexanes), the title compound $4 \mathrm{a}$ was obtained as a colorless oil in $78 \%$ yield $(66.0 \mathrm{mg}, 0.156 \mathrm{mmol}, 10: 1 \mathrm{dr}, 90 \%$ ee).

Aldehyde oxidation level: Aldehyde $3 a(37.0 \mathrm{mg}, 0.2 \mathrm{mmol})$ was subjected to standard reaction conditions $\left(75^{\circ} \mathrm{C}, 24 \mathrm{~h}\right.$ ). Upon flash column chromatography ( $\mathrm{SiO}_{2}, 5: 95 \mathrm{EtOAc}$ :hexanes), the title compound 4 a was obtained as a colorless oil in $78 \%$ yield $(66.0 \mathrm{mg}, 0.156 \mathrm{mmol}, 11: 1 \mathrm{dr}, 90 \%$ ee).

$\mathrm{TLC}\left(\mathrm{SiO}_{2}\right) \mathrm{R}_{\mathrm{f}}=0.3$ (10:90 EtOAc:hexanes)

${ }^{1} \mathrm{H}$ NMR $\left(500 \mathrm{MHz}, \mathrm{CDCl}_{3}\right) \delta: 7.38-7.35(\mathrm{~m}, 2 \mathrm{H}), 7.25(\mathrm{~m}, 8 \mathrm{H}), 7.20-7.16(\mathrm{~m}, 4 \mathrm{H}), 5.76(\mathrm{dd}, J=$ $17.6,10.9 \mathrm{~Hz}, 1 \mathrm{H}), 5.51(\mathrm{~s}, 1 \mathrm{H}), 5.22(\mathrm{dd}, J=10.9,1.0 \mathrm{~Hz}, 1 \mathrm{H}), 5.03(\mathrm{dd}, J=17.6,1.0 \mathrm{~Hz}, 1 \mathrm{H}), 4.67$ $(\mathrm{d}, J=2.5 \mathrm{~Hz}, 1 \mathrm{H}), 3.29(\mathrm{~d}, J=2.5 \mathrm{~Hz}, 1 \mathrm{H}), 0.97(\mathrm{~s}, 3 \mathrm{H})$.

${ }^{13} \mathrm{C}$ NMR $\left(125 \mathrm{MHz},\left(\mathrm{CD}_{3}\right)_{2} \mathrm{CO}\right) \delta: 146.7,145.9,141.5,140.8,131.4,130.9,129.1,128.9,127.7$, $127.5,127.4,121.4,118.2,83.9,80.0,79.9,77.6,18.9$.

HRMS $(\mathrm{Na}+, \mathrm{m} / z)$ for $\mathrm{C}_{24} \mathrm{H}_{23} \mathrm{BrO}_{2}:$ calcd. $=445.0774 ;$ found $=445.0773$.

FTIR (neat): 3350, 2989, 2361, 1453, 1264, 1010, 733, $702 \mathrm{~cm}^{-1}$

HPLC: (Two connected Chiralcel AD-H columns, Hexane:2-PrOH = 98:02, $1.0 \mathrm{~mL} / \mathrm{min}, 210 \mathrm{~nm}$ )

$[\alpha]_{D}^{24}=-7.3^{\circ}\left(\mathrm{c}=0.41, \mathrm{CHCl}_{3}\right)$. 


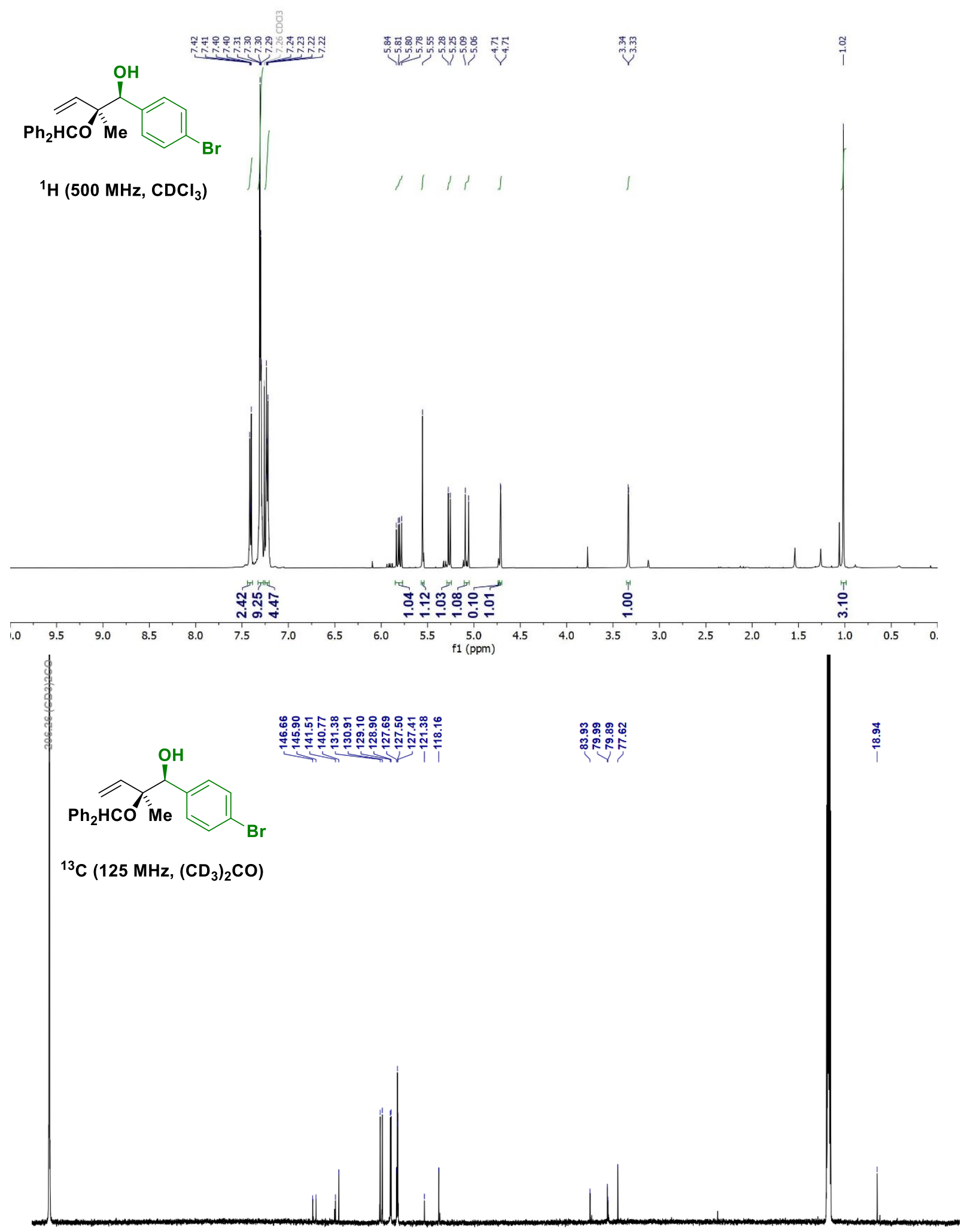

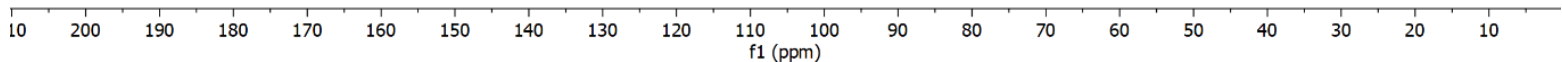




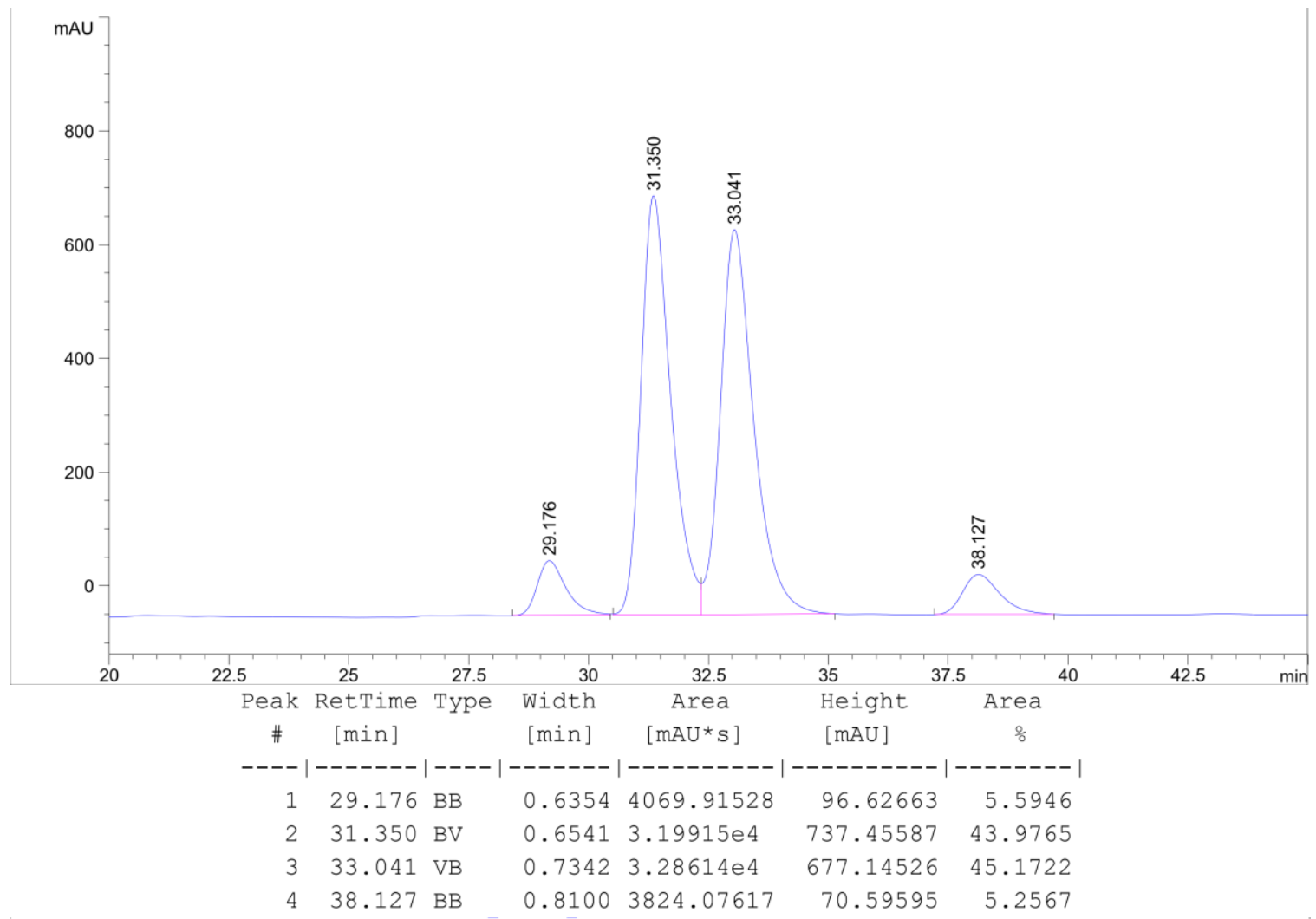

$\mathrm{mAU}]$

\section{From Alcohol}

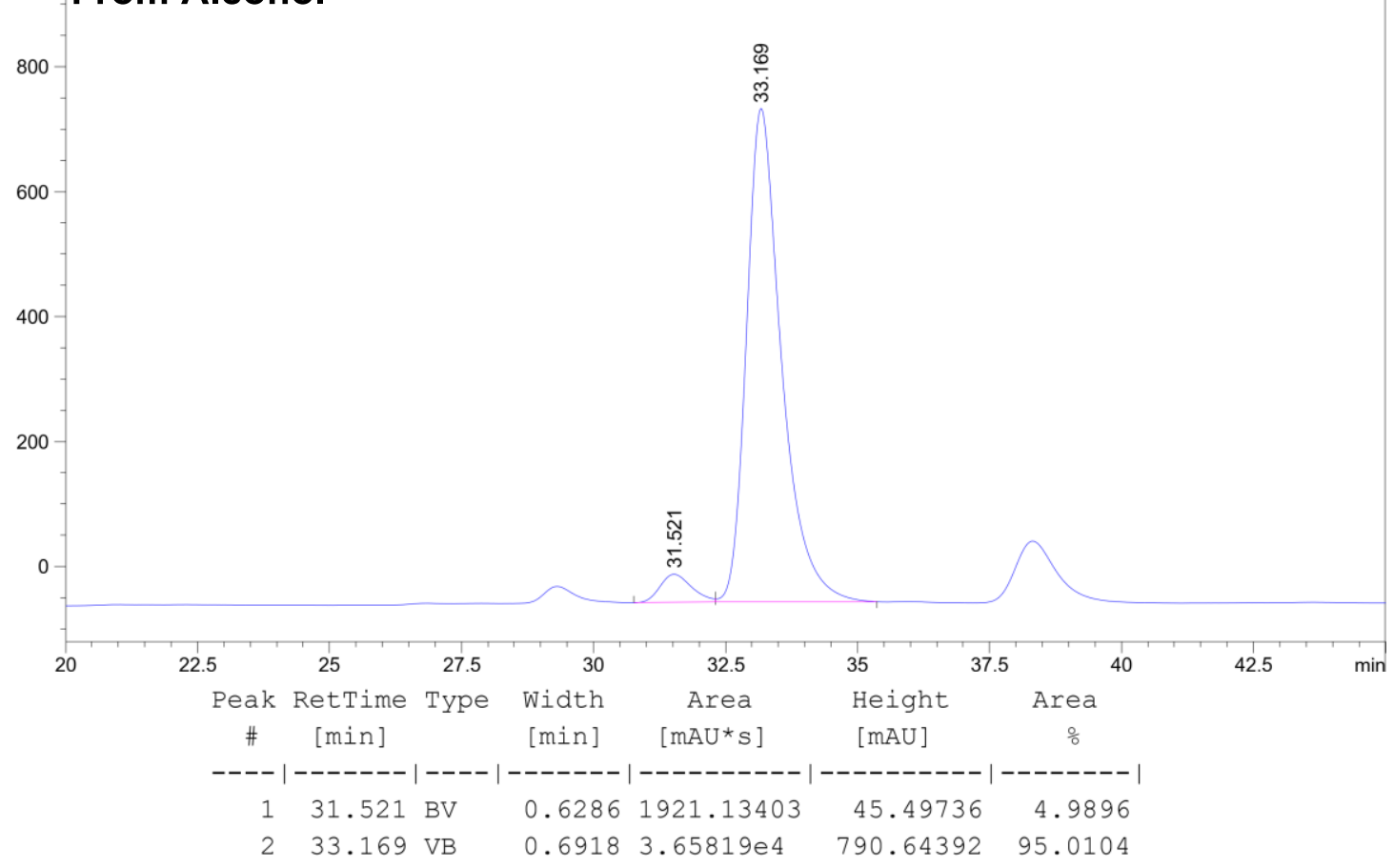




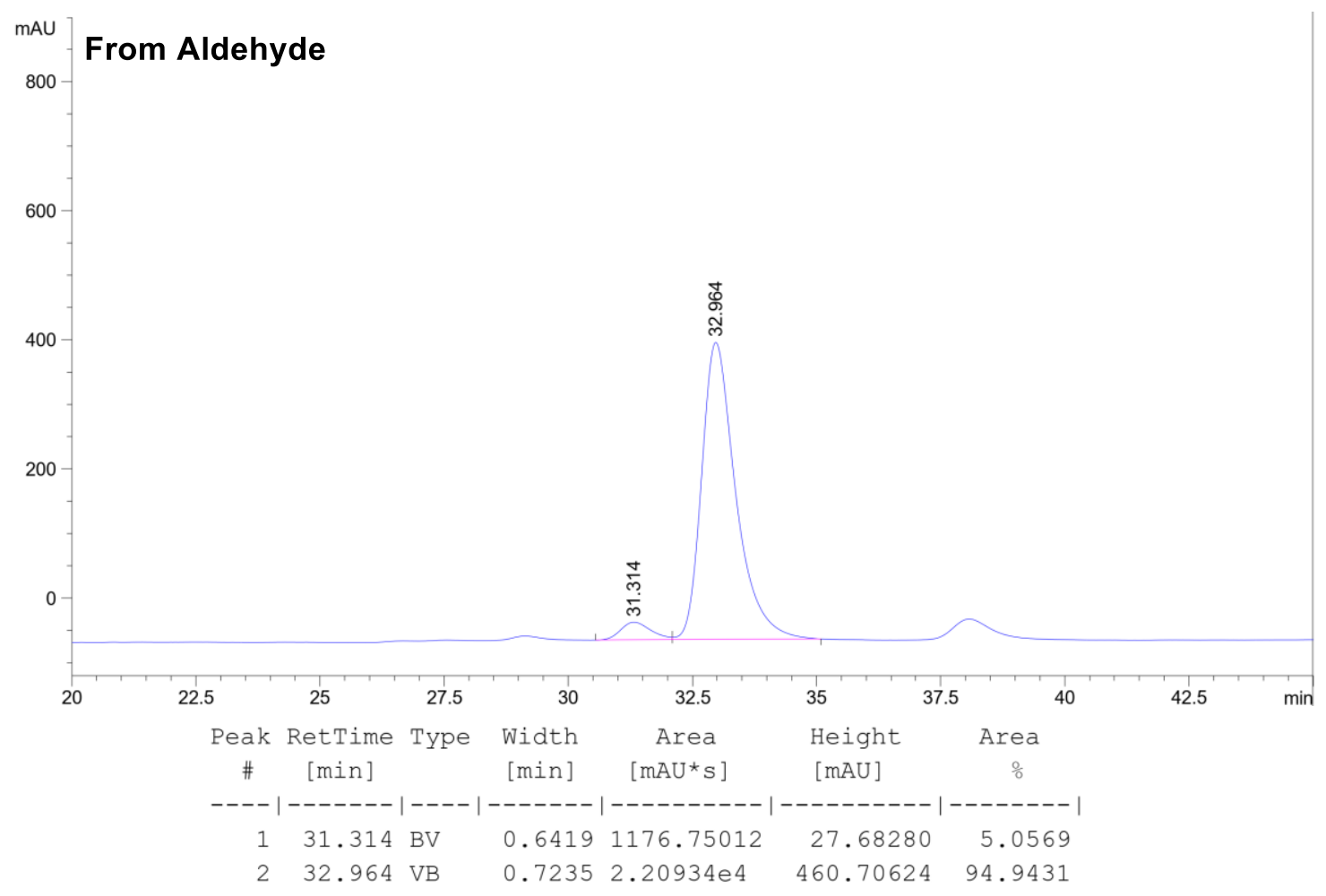


(1S,2S)-2-(benzhydryloxy)-2-methyl-1-(4-(4,4,5,5-tetramethyl-1,3,2-dioxaborolan-2-yl)phenyl) but-3-en-1-ol (4b)

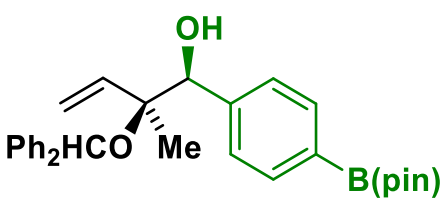

Alcohol oxidation level: Alcohol $\mathbf{2 b}(46.8 \mathrm{mg}, 0.2 \mathrm{mmol})$ was subjected to standard reaction conditions $\left(75^{\circ} \mathrm{C}, 24 \mathrm{~h}\right)$. Upon flash column chromatography $\left(\mathrm{SiO}_{2}, 5: 95 \mathrm{EtOAc}\right.$ :hexanes), the title compound $\mathbf{4 b}$ was obtained as a colorless crystalline solid in $77 \%$ yield $(72.4 \mathrm{mg}, 0.154 \mathrm{mmol}, 6: 1$ dr, $98 \%$ ee).

Aldehyde oxidation level: Aldehyde $3 \mathbf{b}(46.4 \mathrm{mg}, 0.2 \mathrm{mmol})$ was subjected to standard reaction conditions $\left(75^{\circ} \mathrm{C}, 24 \mathrm{~h}\right)$. Upon flash column chromatography ( $\mathrm{SiO}_{2}, 5: 95 \mathrm{EtOAc}$ :hexanes), the title compound $\mathbf{4 b}$ was obtained as a colorless crystalline solid in $70 \%$ yield $(65.8 \mathrm{mg}, 0.14 \mathrm{mmol}, 7: 1$ dr, $94 \%$ ee).

$\operatorname{TLC}\left(\mathrm{SiO}_{2}\right) \mathrm{R}_{\mathrm{f}}=0.5$ (10:90 EtOAc:hexanes)

${ }^{1} \mathbf{H}$ NMR $\left(400 \mathrm{MHz}, \mathrm{CDCl}_{3}\right) \delta: 7.73-7.60(\mathrm{~m}, 2 \mathrm{H}), 7.35-7.26(\mathrm{~m}, 5 \mathrm{H}), 7.25-7.20(\mathrm{~m}, 5 \mathrm{H}), 7.19-$ $7.13(\mathrm{~m}, 2 \mathrm{H}), 5.78(\mathrm{dd}, \mathrm{J}=17.6,10.9 \mathrm{~Hz}, 1 \mathrm{H}), 5.51(\mathrm{~d}, \mathrm{~J}=2.3 \mathrm{~Hz}, 1 \mathrm{H}), 5.18(\mathrm{dd}, J=10.9,1.0 \mathrm{~Hz}$, $1 \mathrm{H}), 4.99(\mathrm{dd}, J=17.7,1.1 \mathrm{~Hz}, 1 \mathrm{H}), 4.72(\mathrm{~d}, \mathrm{~J}=2.2 \mathrm{~Hz}, 1 \mathrm{H}), 3.32(\mathrm{~d}, \mathrm{~J}=2.6 \mathrm{~Hz}, 1 \mathrm{H}), 1.29(\mathrm{~s}, 12 \mathrm{H})$, $0.97(\mathrm{~s}, 3 \mathrm{H})$.

${ }^{13} \mathrm{C}$ NMR $\left(100 \mathrm{MHz}_{2} \mathrm{CDCl}_{3}\right) \delta: 144.7,144.1,142.3,139.9,134.0,128.6,128.4,127.4,127.4,127.3$, $127.2,126.8,126.7,118.7,83.9,80.1,77.4,77.1,25.0,16.6$.

HRMS $(\mathrm{Na}+, \mathrm{m} / z)$ for $\mathrm{C}_{30} \mathrm{H}_{35} \mathrm{BO}_{4}$ : calcd. $=492.2557$; found $=492.2556$.

FTIR (neat): 3563, 2978, 1613, 1493, 1359, 1267, 1143, $1088 \mathrm{~cm}^{-1}$.

HPLC: (Two connected Chiralcel columns AD-H and OD-H, Hexane:2-PrOH = 98:02, $0.5 \mathrm{~mL} / \mathrm{min}$, $210 \mathrm{~nm}$ )

$[\alpha]_{D}^{24}=-63.0^{\circ}\left(\mathrm{c}=0.2, \mathrm{CHCl}_{3}\right)$.

MP: [150-158] ${ }^{\circ} \mathrm{C}$ 


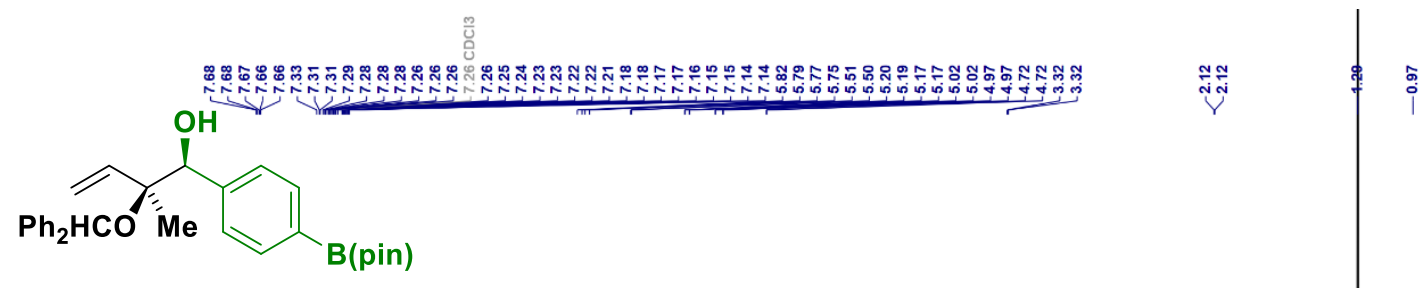

${ }^{1} \mathrm{H}\left(400 \mathrm{MHz}, \mathrm{CDCl}_{3}\right)$
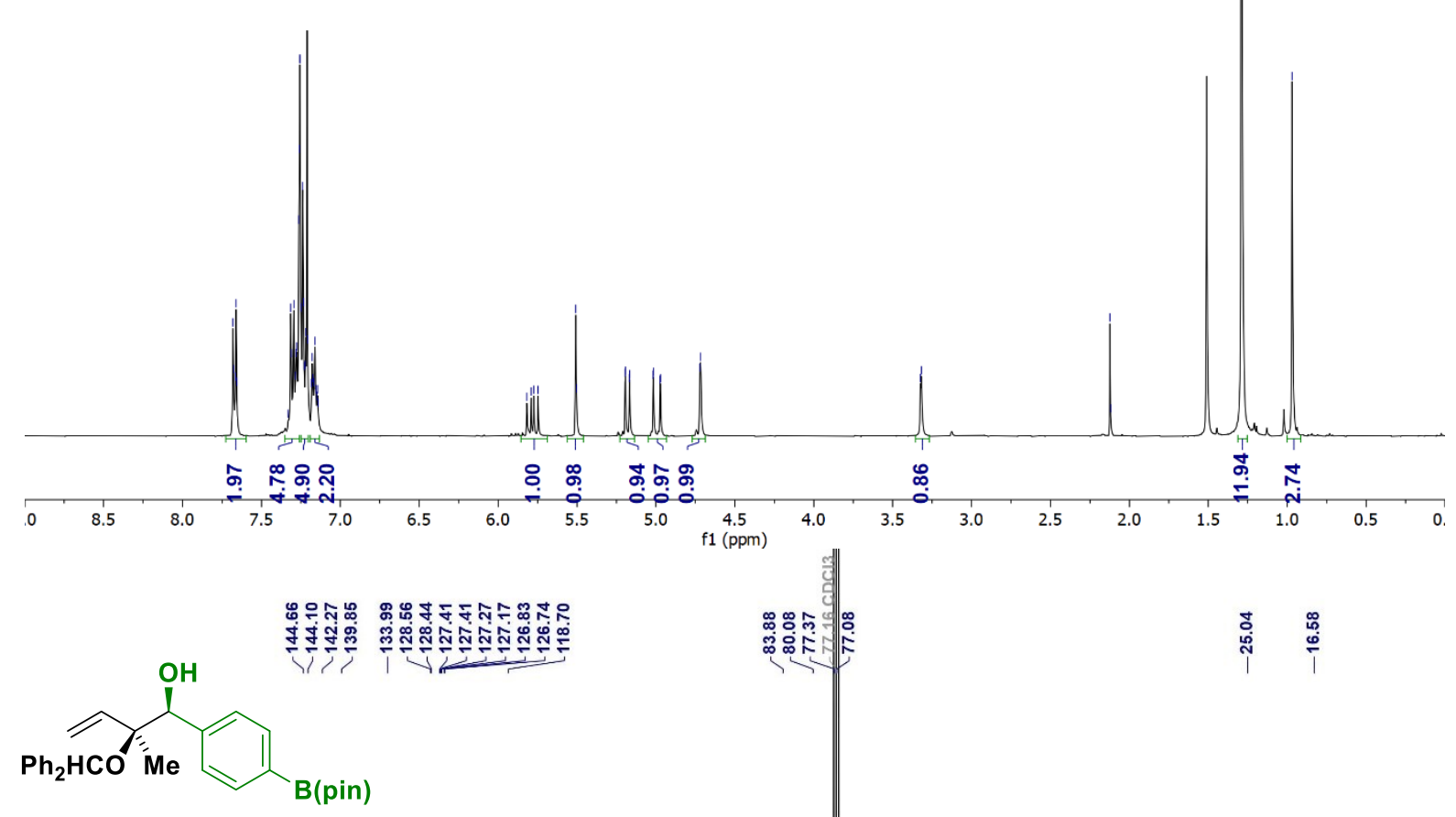

${ }^{13} \mathrm{C}\left(100 \mathrm{MHz}, \mathrm{CDCl}_{3}\right)$

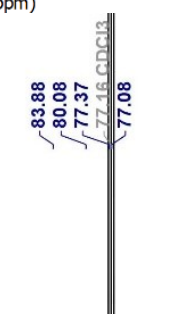

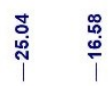

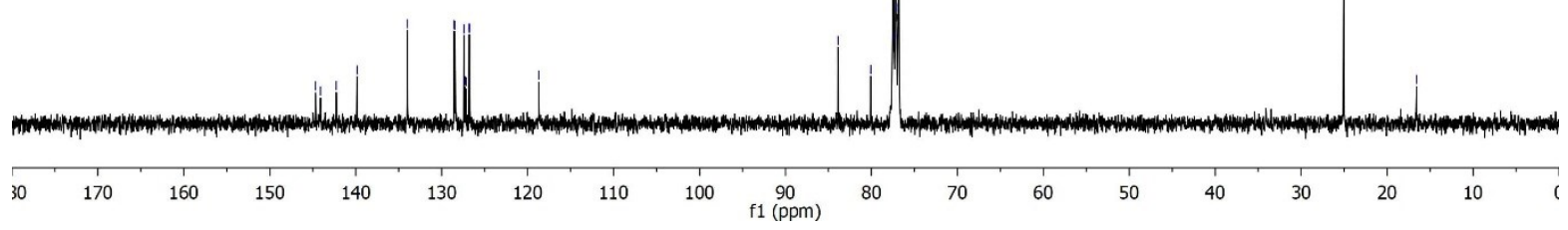



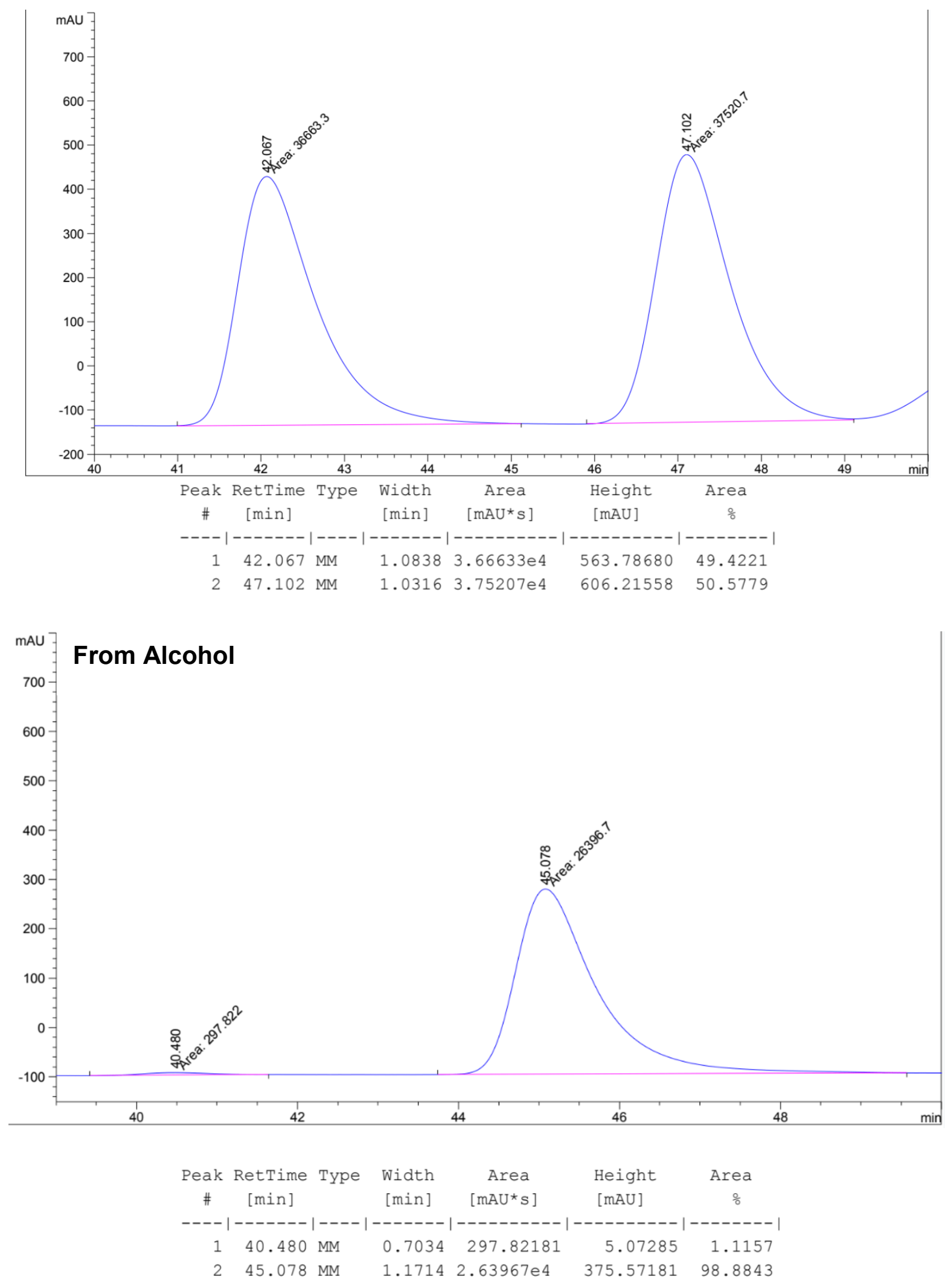


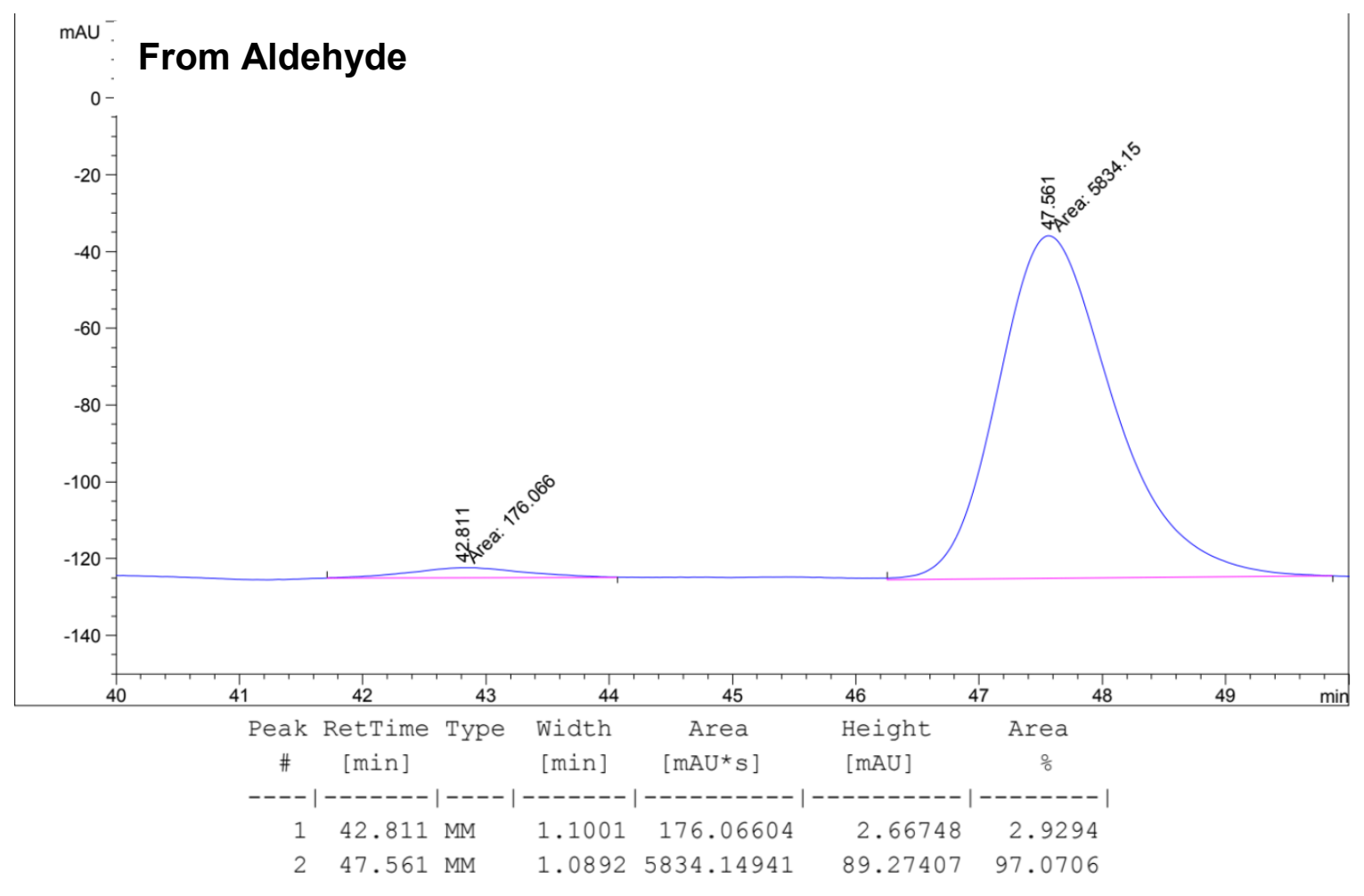




\section{(1S,2S)-2-(benzhydryloxy)-1-(4-chloro-3-methylphenyl)-2-methylbut-3-en-1-ol (4c)}<smiles>C=C[C@](C)(OC)[C@H](O)c1ccc(Cl)c(C)c1</smiles>

Alcohol oxidation level: Alcohol $2 \mathrm{c}(31.3 \mathrm{mg}, 0.2 \mathrm{mmol})$ was subjected to standard reaction conditions $\left(75^{\circ} \mathrm{C}, 24 \mathrm{~h}\right)$. Upon flash column chromatography $\left(\mathrm{SiO}_{2}, 5: 95\right.$ EtOAc:hexanes), the title compound $4 \mathrm{c}$ was obtained as a yellow oil in $78 \%$ yield $(61.4 \mathrm{mg}, 0.156 \mathrm{mmol}, 7: 1 \mathrm{dr}, 94 \%$ ee).

Aldehyde oxidation level: Aldehyde $3 \mathrm{c}(30.9 \mathrm{mg}, 0.2 \mathrm{mmol})$ was subjected to standard reaction conditions $\left(75^{\circ} \mathrm{C}, 24 \mathrm{~h}\right)$. Upon flash column chromatography $\left(\mathrm{SiO}_{2}, 5: 95 \mathrm{EtOAc}\right.$ :hexanes), the title compound $4 \mathrm{c}$ was obtained as a yellow oil $84 \%$ yield $(65.8 \mathrm{mg}, 0.168 \mathrm{mmol}, 9: 1 \mathrm{dr}, 92 \%$ ee).

$\operatorname{TLC}\left(\mathrm{SiO}_{2}\right) \mathrm{R}_{\mathrm{f}}=0.2$ (10:90 EtOAc:hexanes)

${ }^{1}$ H NMR (500 MHz, DMSO) $\delta: 7.46-7.42(\mathrm{~m}, 2 \mathrm{H}), 7.38-7.33(\mathrm{~m}, 1 \mathrm{H}), 7.30-7.24(\mathrm{~m}, 5 \mathrm{H}), 7.22-$ $7.14(\mathrm{~m}, 4 \mathrm{H}), 7.13-7.09(\mathrm{~m}, 1 \mathrm{H}), 5.59(\mathrm{ddd}, J=17.7,11.0,1.5 \mathrm{~Hz}, 1 \mathrm{H}), 5.52(\mathrm{~s}, 1 \mathrm{H}), 5.49(\mathrm{~d}, J=$ $4.7 \mathrm{~Hz}, 1 \mathrm{H}), 5.11(\mathrm{dd}, J=10.9,1.6 \mathrm{~Hz}, 1 \mathrm{H}), 5.01(\mathrm{dd}, J=17.7,1.6 \mathrm{~Hz}, 1 \mathrm{H}), 4.57(\mathrm{~d}, J=4.7 \mathrm{~Hz}, 1 \mathrm{H})$, $2.28(\mathrm{~s}, 3 \mathrm{H}), 1.10(\mathrm{~s}, 3 \mathrm{H})$.

${ }^{13} \mathrm{C}$ NMR (125 MHz, DMSO) $\delta: 145.7,145.0,140.8,139.5,133.4,131.5,131.3,128.1,127.9,127.5$, $127.2,126.6,126.5,126.2,126.1,116.9,82.3,78.1,75.8,19.6,19.6$.

HRMS $(\mathrm{Na}+, m / z)$ for $\mathrm{C}_{25} \mathrm{H}_{25} \mathrm{ClO}_{2}$ : calcd. $=415.1435$; found $=415.1423$.

FTIR (neat): 3558, 3026, 1452, 1157, 1044, 1024, 929, 740, $697 \mathrm{~cm}^{-1}$

HPLC: (Two connected Chiralcel columns AD-H and OD-H, Hexane:2-PrOH = 98:02, $0.5 \mathrm{~mL} / \mathrm{min}$, $210 \mathrm{~nm}$ )

$[\alpha]_{D}^{24}=-19.0^{\circ}\left(\mathrm{c}=1.0, \mathrm{CHCl}_{3}\right)$ 


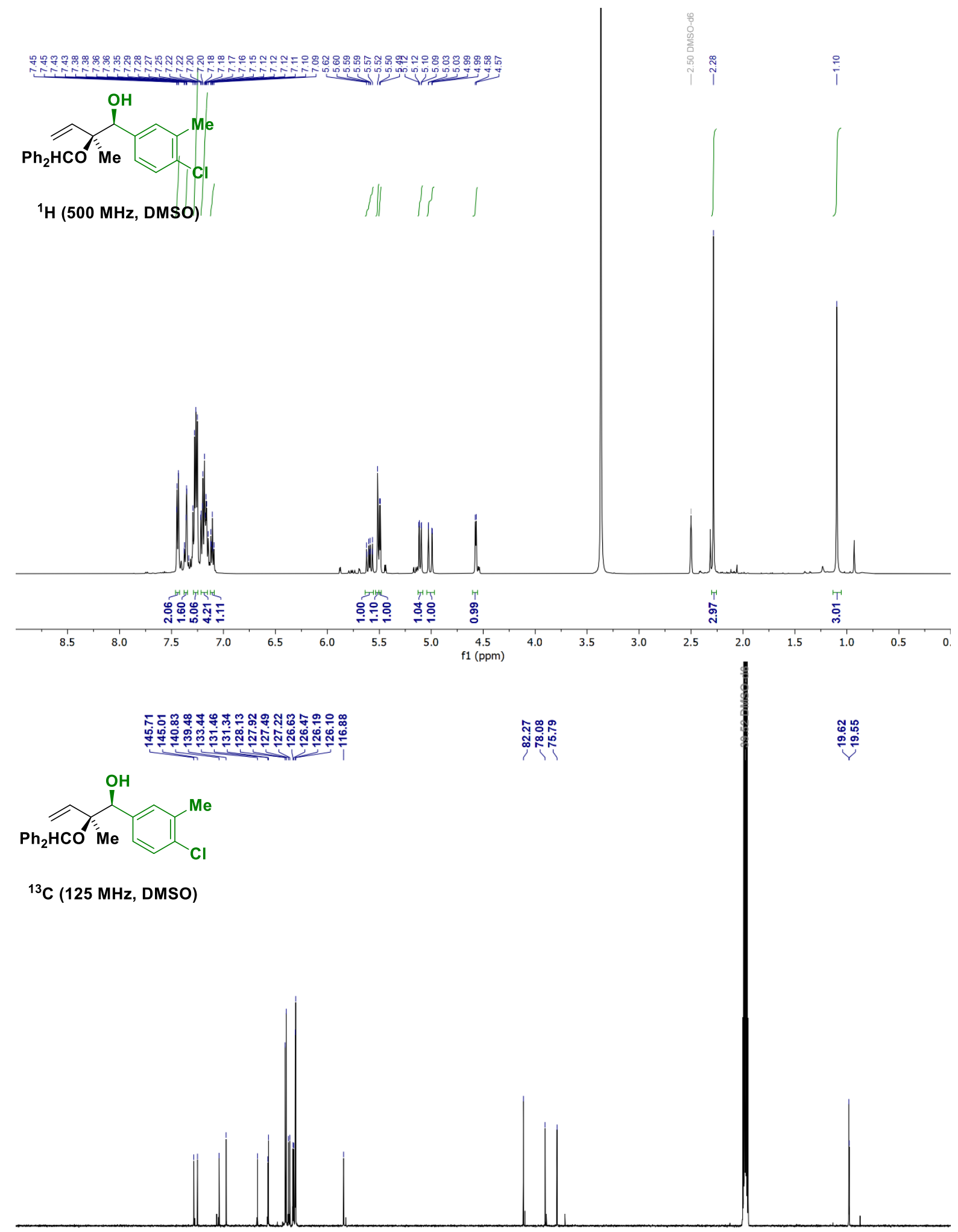

${ }^{1} \mathrm{H}$ (500 MHz, DMSO)

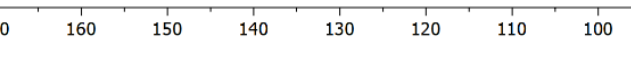

90
$\mathrm{f} 1(\mathrm{ppm})$ 

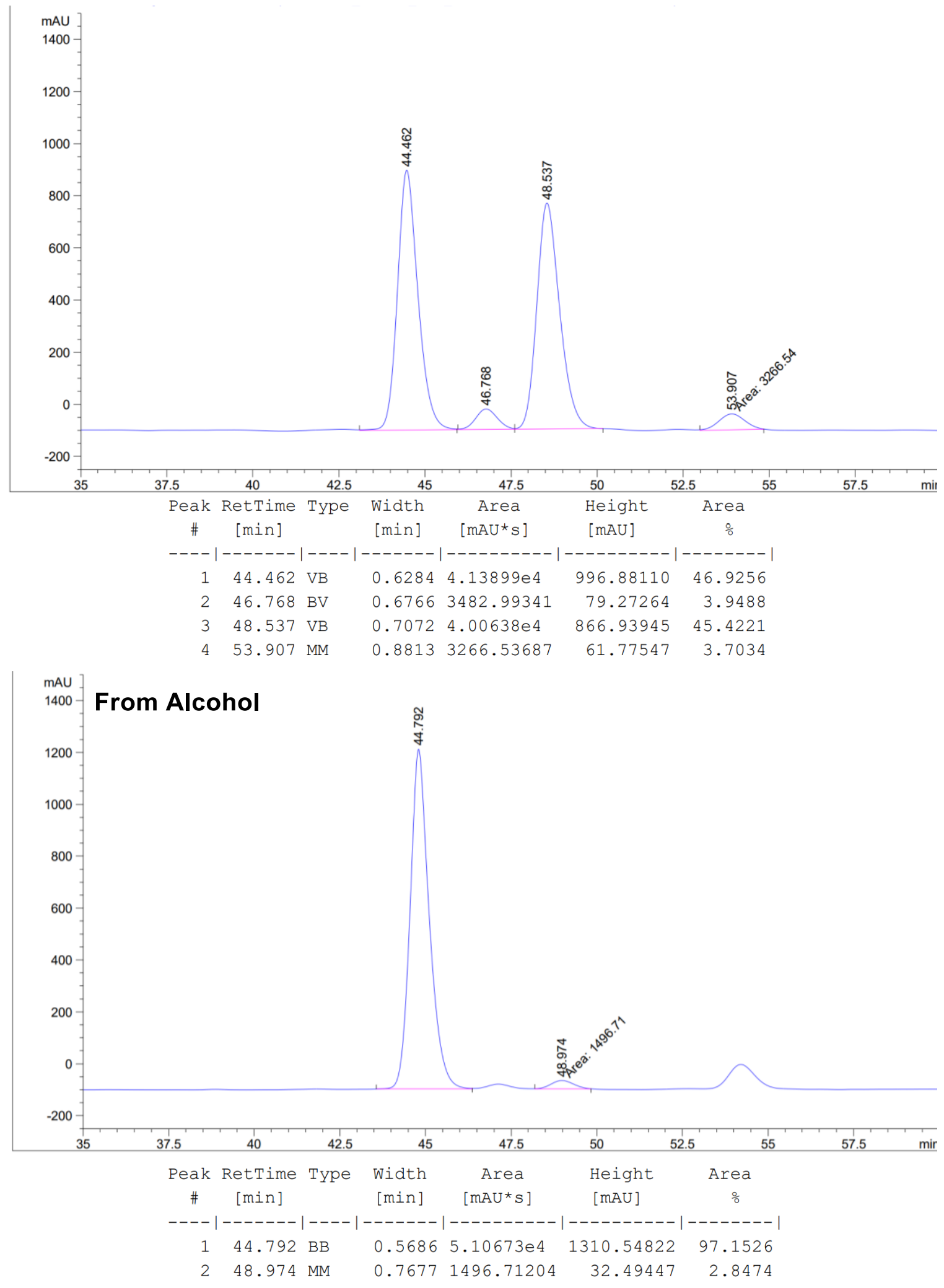


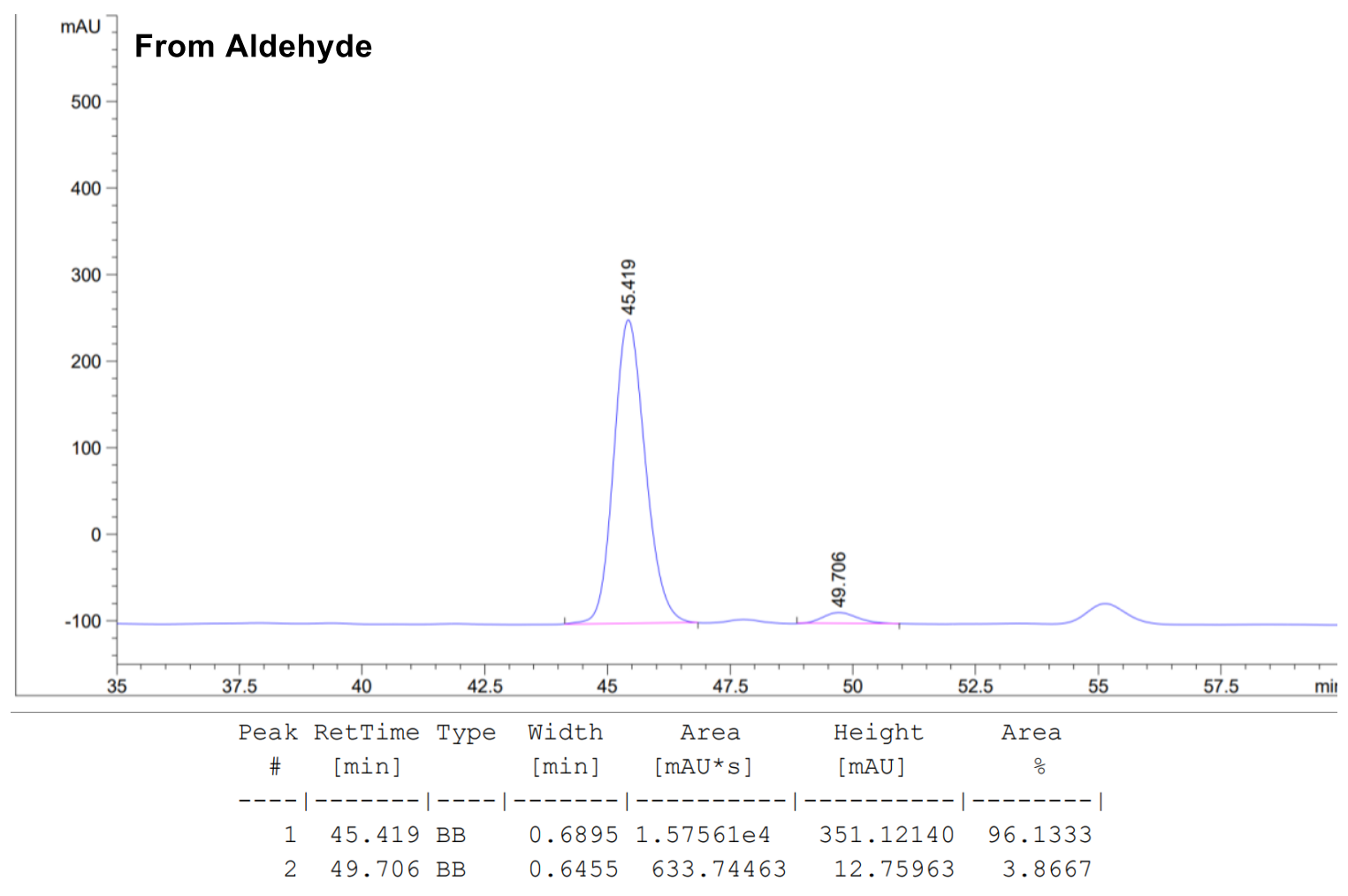


(1S,2S)-2-(benzhydryloxy)-1-(4-fluoro-3-methoxyphenyl)-2-methylbut-3-en-1-ol (4d)<smiles>C=C[C@](C)(OCc1ccccc1)[C@@H](O)c1ccc(F)c(OC)c1</smiles>

Alcohol oxidation level: Aldehyde $\mathbf{2 d}(31.2 \mathrm{mg}, 0.2 \mathrm{mmol})$ was subjected to standard reaction conditions $\left(75^{\circ} \mathrm{C}, 24 \mathrm{~h}\right)$. Upon flash column chromatography $\left(\mathrm{SiO}_{2}, 8: 92 \mathrm{EtOAc}\right.$ :hexanes), the title compound $4 \mathbf{d}$ was obtained as a yellow solid in $95 \%$ yield $(74.6 \mathrm{mg}, 0.190 \mathrm{mmol}, 8: 1 \mathrm{dr}, 96 \%$ ee).

Aldehyde oxidation level: Aldehyde $3 \mathbf{d}(30.8 \mathrm{mg}, 0.2 \mathrm{mmol})$ was subjected to standard reaction conditions $\left(75^{\circ} \mathrm{C}, 24 \mathrm{~h}\right)$. Upon flash column chromatography $\left(\mathrm{SiO}_{2}, 8: 92 \mathrm{EtOAc}\right.$ :hexanes), the title compound $\mathbf{4 d}$ was obtained as a yellow solid in $88 \%$ yield $(69.0 \mathrm{mg}, 0.176 \mathrm{mmol}, 10: 1 \mathrm{dr}, 97 \%$ ee).

$\operatorname{TLC}\left(\mathrm{SiO}_{2}\right) \mathrm{R}_{\mathrm{f}}=0.2(10: 90$ EtOAc:hexanes)

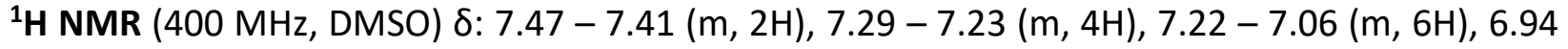
(ddd, $J=8.4,4.6,1.9 \mathrm{~Hz}, 1 \mathrm{H}), 5.67(\mathrm{dd}, J=17.7,10.9 \mathrm{~Hz}, 1 \mathrm{H}), 5.52(\mathrm{~s}, 1 \mathrm{H}), 5.49(\mathrm{~d}, J=4.8 \mathrm{~Hz}, 1 \mathrm{H})$, $5.12(\mathrm{dd}, J=10.9,1.5 \mathrm{~Hz}, 1 \mathrm{H}), 5.03(\mathrm{dd}, J=17.7,1.6 \mathrm{~Hz}, 1 \mathrm{H}), 4.58(\mathrm{~d}, J=4.7 \mathrm{~Hz}, 1 \mathrm{H}), 3.74(\mathrm{~s}, 3 \mathrm{H})$, $1.09(\mathrm{~s}, 3 \mathrm{H})$.

${ }^{13} \mathrm{C}$ NMR $\left(100 \mathrm{MHz}, \mathrm{CDCl}_{3}\right) \delta: 152.0(\mathrm{~d}, J=245.1 \mathrm{~Hz}), 146.9(\mathrm{~d}, J=10.6 \mathrm{~Hz}), 144.6,144.0,139.9$, $135.4(d, J=3.8 \mathrm{~Hz}), 128.6,128.5,127.3,127.2,126.8,126.7,120.6$ (d, $J=6.8 \mathrm{~Hz}), 118.8,114.9$ $(\mathrm{d}, J=18.3 \mathrm{~Hz}), 113.2(\mathrm{~d}, J=1.9 \mathrm{~Hz}), 83.7,79.5,77.1,56.3,16.7$.

${ }^{19}$ F NMR (390 MHz, DMSO) $\delta:-138.2$ (ddd, $J=12.0,8.5,4.4 \mathrm{~Hz}$ ).

HRMS $(\mathrm{Na}+, \mathrm{m} / z)$ for $\mathrm{C}_{25} \mathrm{H}_{25} \mathrm{FO}_{3}$ : calcd. = 415.1680; found $=415.1669$.

FTIR (neat): 3558, 2936, 1611, 1516, 1276, 1025, 742, $700 \mathrm{~cm}^{-1}$

HPLC: (Chiralcel AD-H column, Hexane:2-PrOH = 99:01, $0.5 \mathrm{~mL} / \mathrm{min}, 210 \mathrm{~nm}$ )

$[\alpha]_{D}^{24}=-13.0^{\circ}\left(\mathrm{c}=0.2, \mathrm{CHCl}_{3}\right)$ 


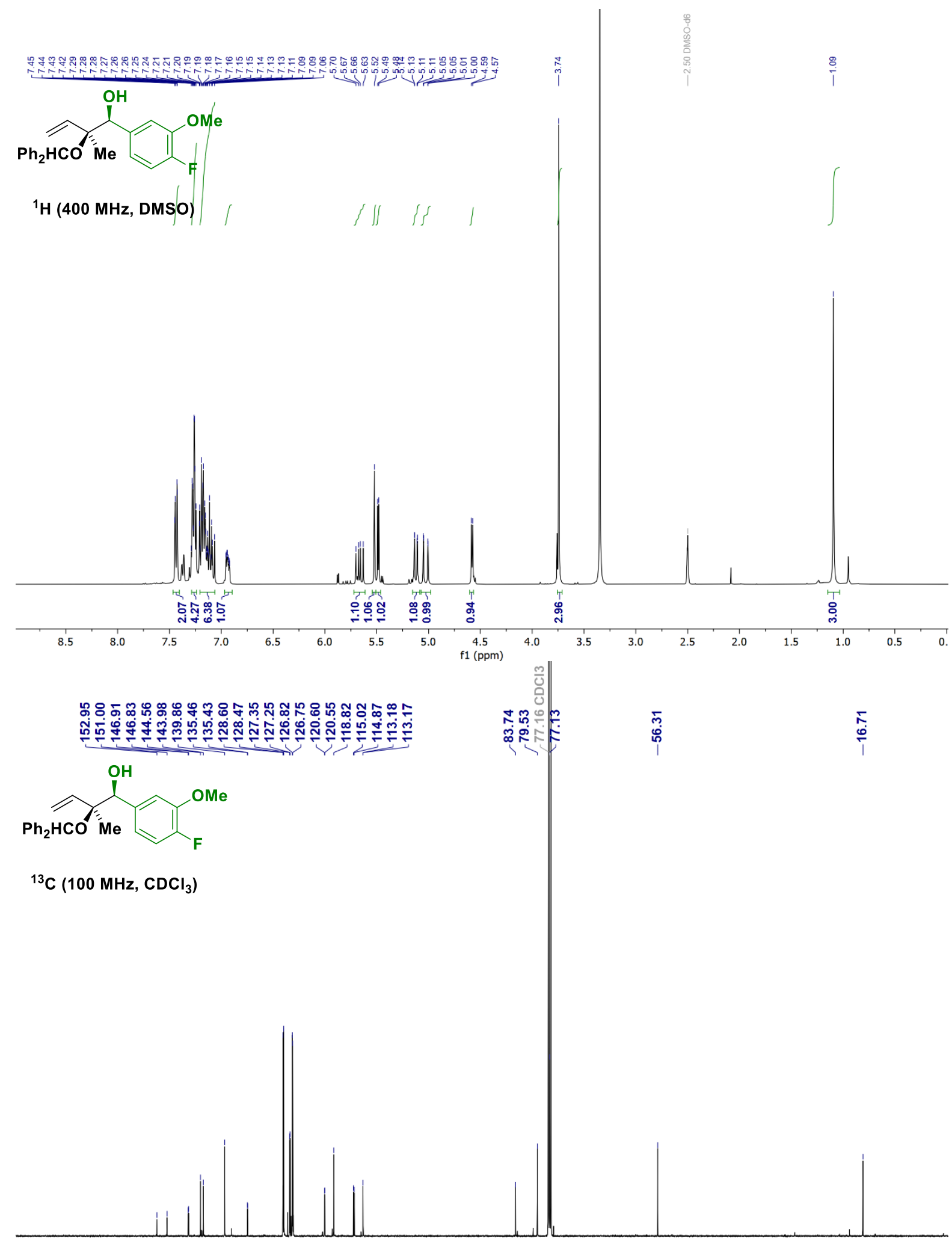

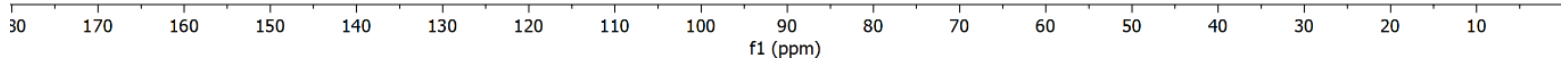



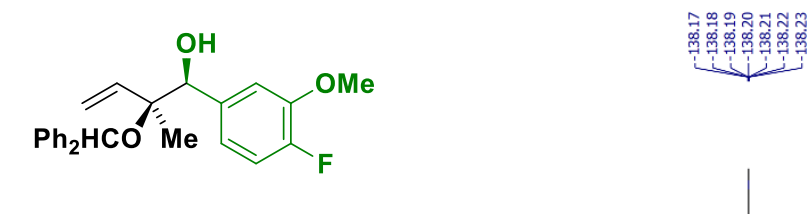

${ }^{19} \mathrm{~F}(390 \mathrm{MHz}, \mathrm{DMSO})$

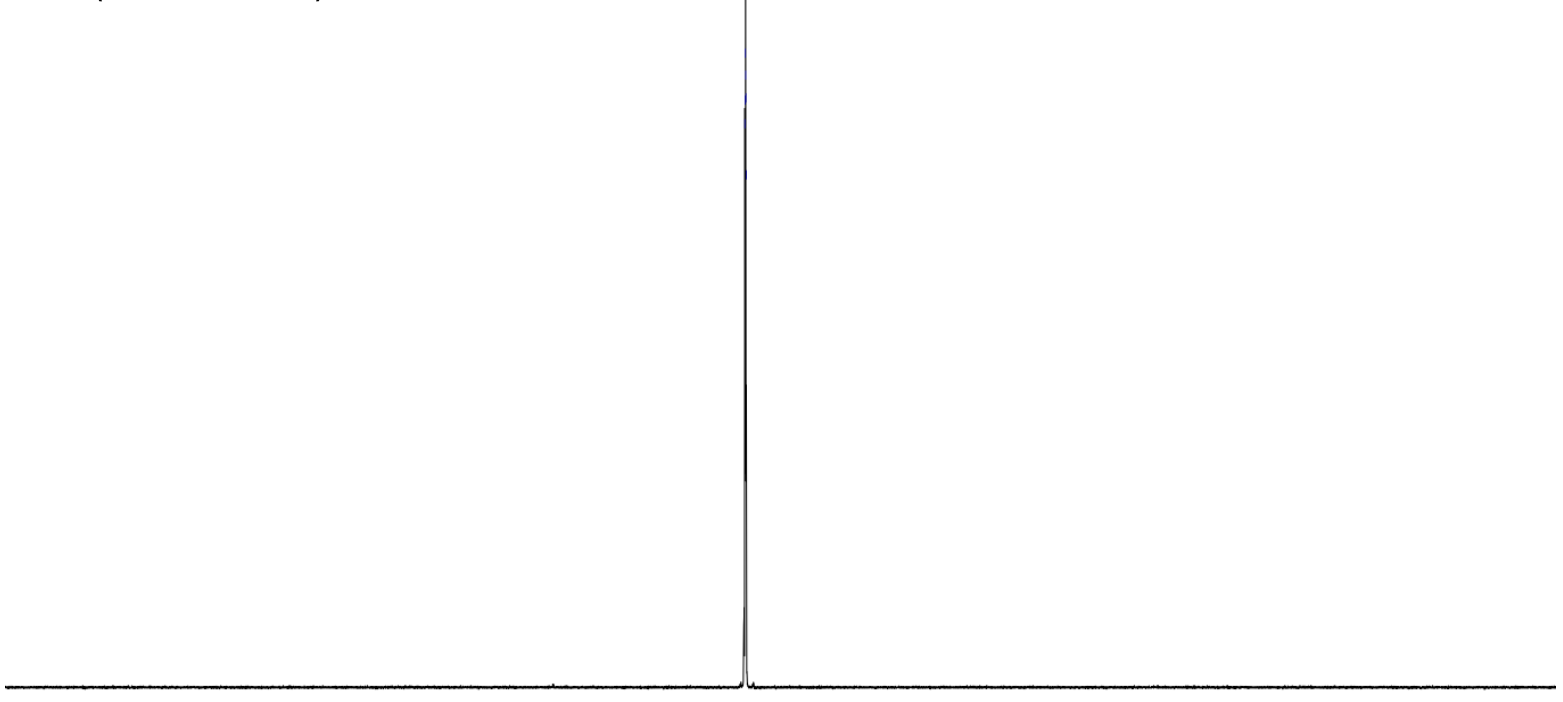

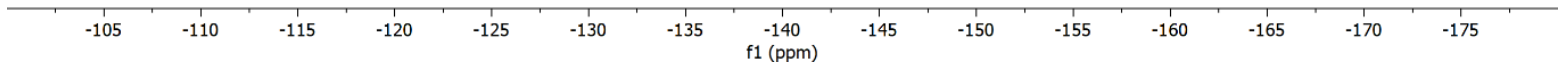



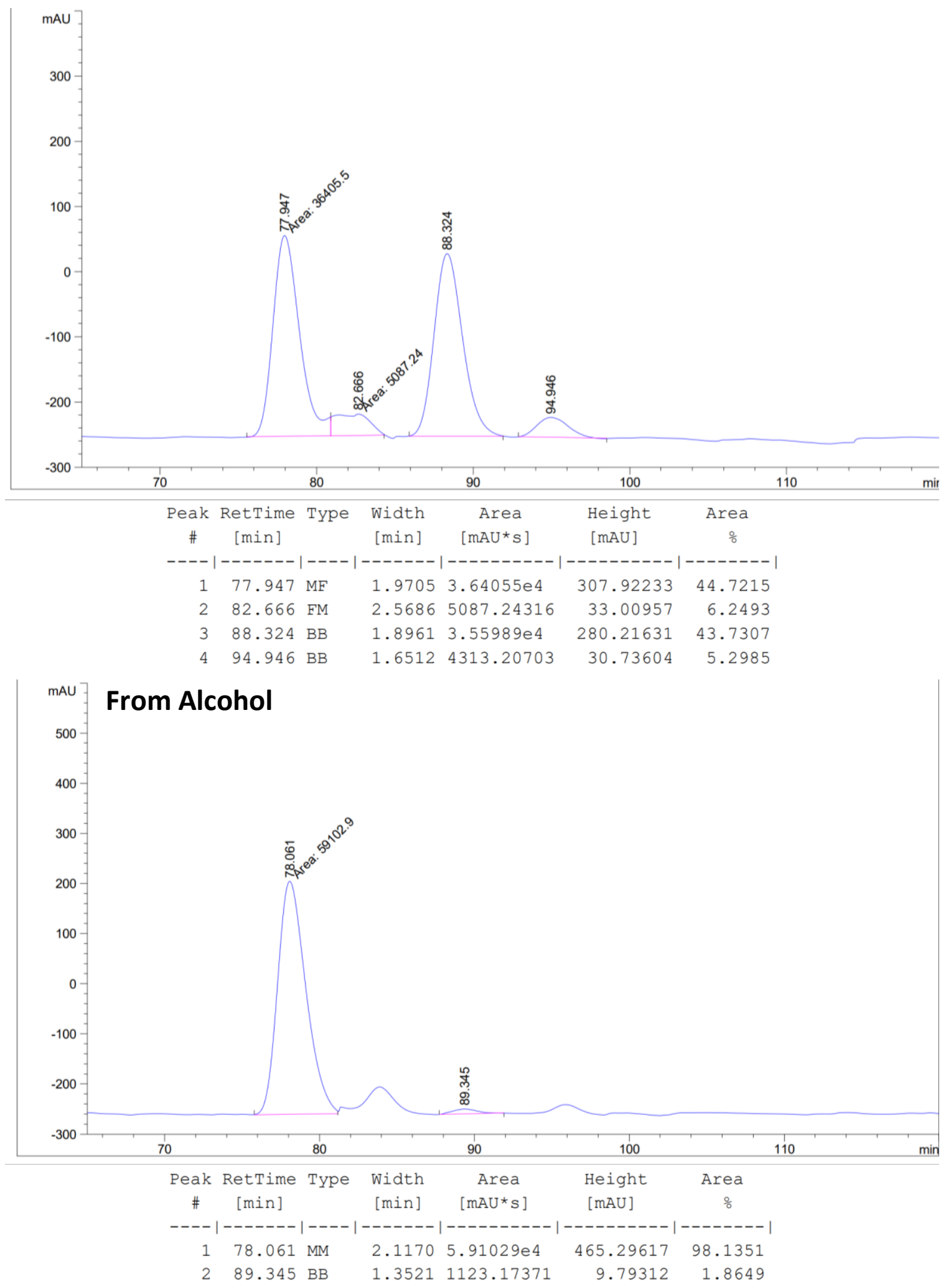


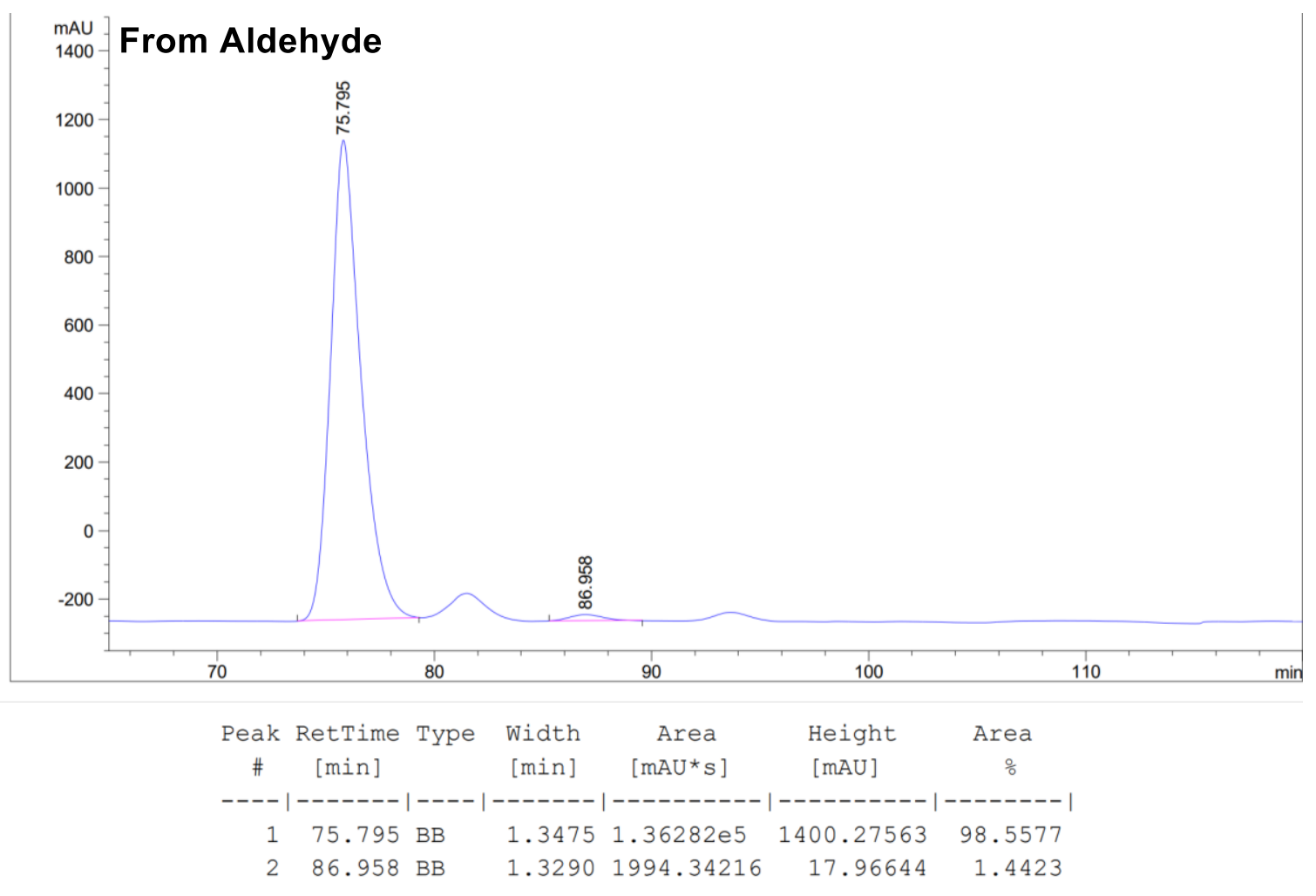




\section{(1S,2S)-2-(benzhydryloxy)-1-(2-fluoro-4-methylphenyl)-2-methylbut-3-en-1-ol (4e)}

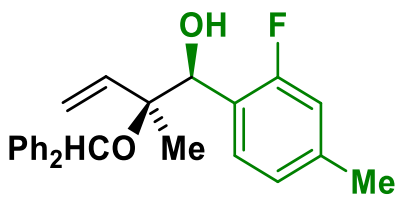

Alcohol oxidation level: Alcohol $2 \mathrm{e}(28.0 \mathrm{mg}, 0.2 \mathrm{mmol})$ was subjected to standard reaction conditions $\left(75^{\circ} \mathrm{C}, 24 \mathrm{~h}\right)$. Upon flash column chromatography $\left(\mathrm{SiO}_{2}, 5: 95 \mathrm{EtOAc}\right.$ :hexanes), the title compound $4 \mathrm{e}$ was obtained as a colorless oil in $85 \%$ yield $(64.0 \mathrm{mg}, 0.17 \mathrm{mmol}, 11: 1 \mathrm{dr}, 91 \%$ ee).

Aldehyde oxidation level: Aldehyde $3 e(27.6 \mathrm{mg}, 0.2 \mathrm{mmol})$ was subjected to standard reaction conditions $\left(75^{\circ} \mathrm{C}, 24 \mathrm{~h}\right)$. Upon flash column chromatography $\left(\mathrm{SiO}_{2}, 5: 95 \mathrm{EtOAc}\right.$ :hexanes), the title compound $4 \mathrm{e}$ was obtained as a colorless oil in $95 \%$ yield $(71.5 \mathrm{mg}, 0.19 \mathrm{mmol}, 15: 1 \mathrm{dr}, 90 \%$ ee).

$\operatorname{TLC}\left(\mathrm{SiO}_{2}\right) \mathrm{R}_{\mathrm{f}}=0.3(7: 93$ EtOAc:hexanes)

${ }^{1} \mathrm{H}$ NMR $\left(400 \mathrm{MHz}, \mathrm{CDCl}_{3}\right) \delta: 7.42(\mathrm{t}, J=7.7 \mathrm{~Hz}, 1 \mathrm{H}), 7.33-7.26(\mathrm{~m}, 8 \mathrm{H}), 7.21$ (ddq, J= 8.0, 5.4, 2.6 $\mathrm{Hz}, 2 \mathrm{H}), 6.95(\mathrm{dd}, J=7.9,1.6 \mathrm{~Hz}, 1 \mathrm{H}), 6.81-6.75(\mathrm{~m}, 1 \mathrm{H}), 6.00-5.91(\mathrm{~m}, 1 \mathrm{H}), 5.55(\mathrm{~s}, 1 \mathrm{H}), 5.25-$ $5.06(\mathrm{~m}, 2 \mathrm{H}), 3.21(\mathrm{~s}, 1 \mathrm{H}), 2.33(\mathrm{~s}, 3 \mathrm{H}), 1.09(\mathrm{~s}, 3 \mathrm{H})$.

${ }^{13} \mathrm{C}$ NMR $\left(100 \mathrm{MHz}, \mathrm{CDCl}_{3}\right) \delta: 160.2(\mathrm{~d}, J=245.8 \mathrm{~Hz}), 144.7,144.2,139.8,139.6(\mathrm{~d}, J=8.2 \mathrm{~Hz})$, $129.11(\mathrm{~d}, J=4.5 \mathrm{~Hz}), 128.5,128.5,127.2,127.2,126.9,126.7,124.48(\mathrm{~d}, J=3.0 \mathrm{~Hz}), 123.7(\mathrm{~d}, J=$ $12.9 \mathrm{~Hz}), 118.2,115.6(\mathrm{~d}, J=22.6 \mathrm{~Hz}), 83.7,77.1,73.1,21.2(\mathrm{~d}, J=1.6 \mathrm{~Hz}), 16.9$.

${ }^{19}$ F NMR $\left(390 \mathrm{MHz}, \mathrm{CDCl}_{3}\right) \delta:-116.0$ (ddd, $\left.J=10.4,7.5,2.3 \mathrm{~Hz}\right)$.

HRMS $(\mathrm{Na}+, m / z)$ for $\mathrm{C}_{25} \mathrm{H}_{25} \mathrm{FO}_{2}$ : calcd. $=399.1744 ;$ found $=399.1744$.

FTIR (neat): 3552, 3062, 3027, 1628, 1599, 1413, 1266, 1110, $1024 \mathrm{~cm}^{-1}$.

HPLC: (Two connected Chiralcel columns AD-H and OD-H, Hexane:2-PrOH = 98:02, $0.5 \mathrm{~mL} / \mathrm{min}$, $210 \mathrm{~nm})$

$[\alpha]_{D}^{24}=-31.0^{\circ}\left(\mathrm{c}=0.1, \mathrm{CHCl}_{3}\right)$. 


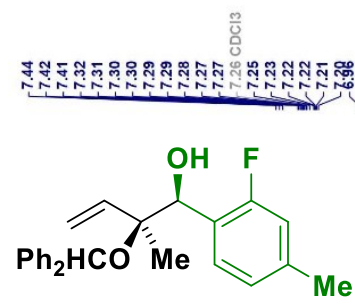

${ }^{1} \mathrm{H}\left(400 \mathrm{MHz}, \mathrm{CDCl}_{3}\right)$
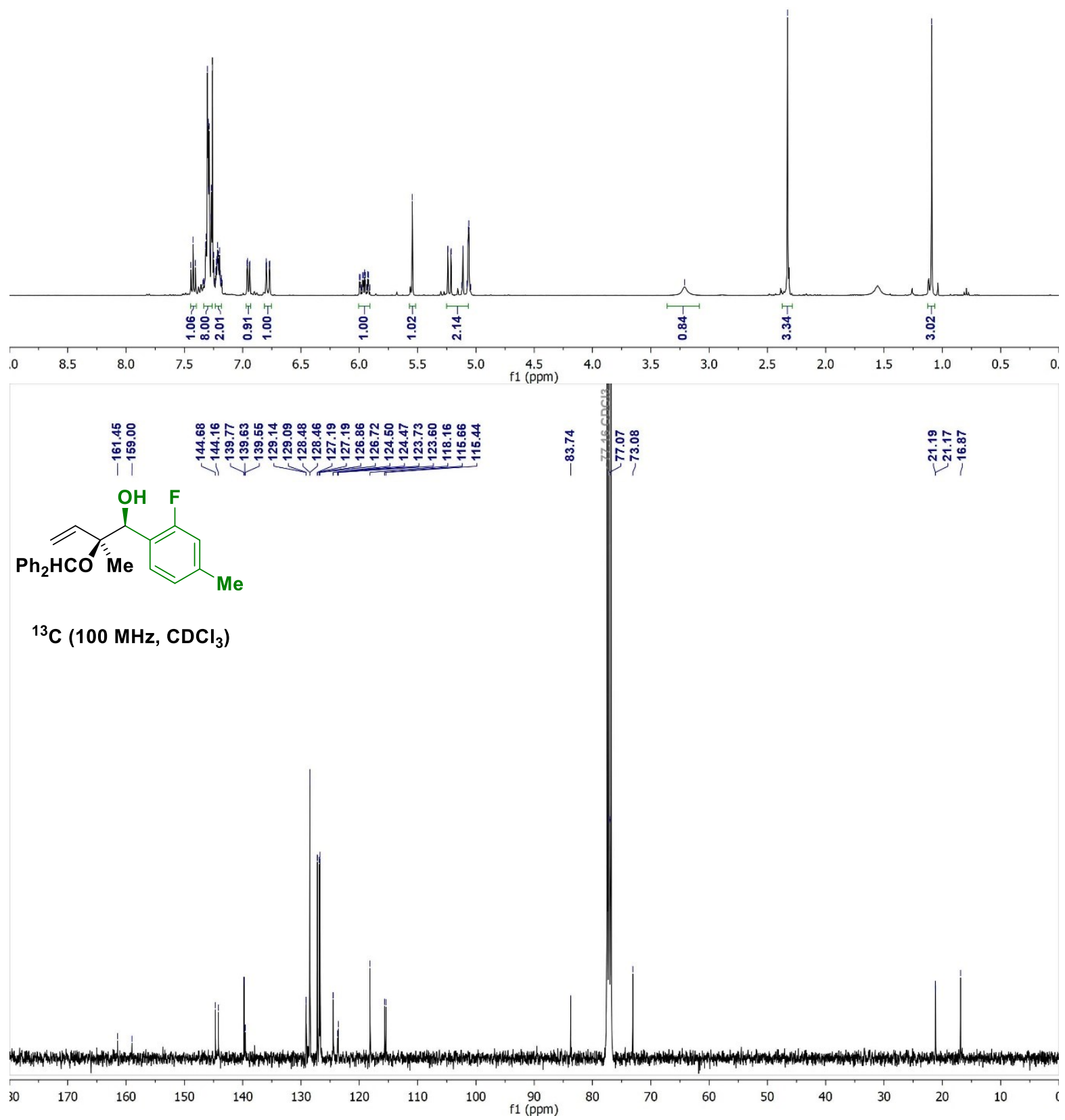

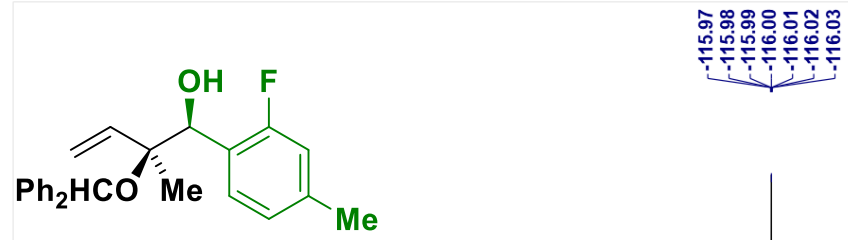

${ }^{19} \mathrm{~F}\left(370 \mathrm{MHz}, \mathrm{CDCl}_{3}\right)$

$\begin{array}{llllllllllllllllllllllllllllllllll}-92 & -94 & -96 & -98 & -100 & -102 & -104 & -106 & -108 & -110 & -112 & -114 & -116 & -118 & -120 & -122 & -124 & -126 & -128 & -130 & -132 & -134 & -136 & -138 & -140\end{array}$



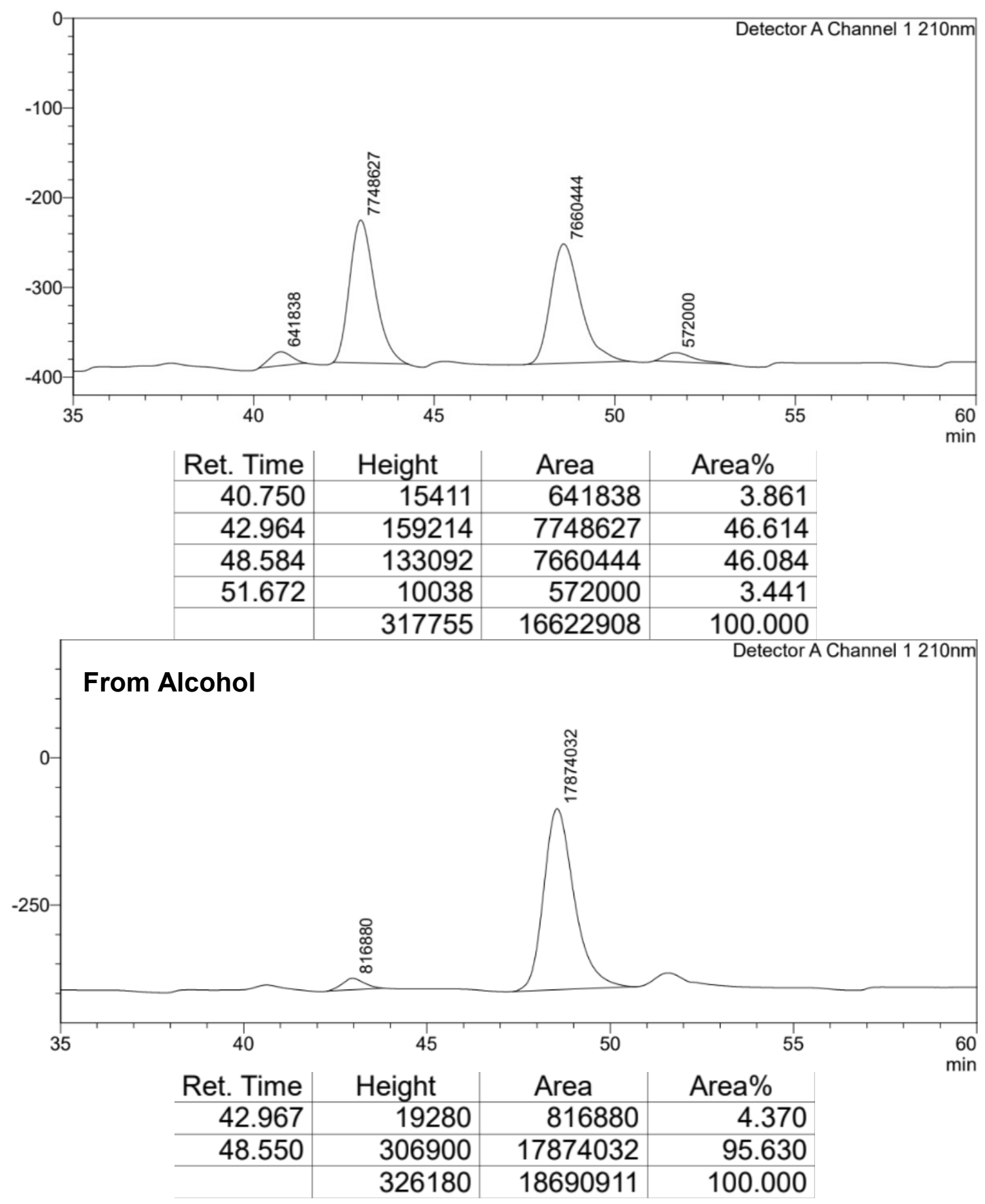


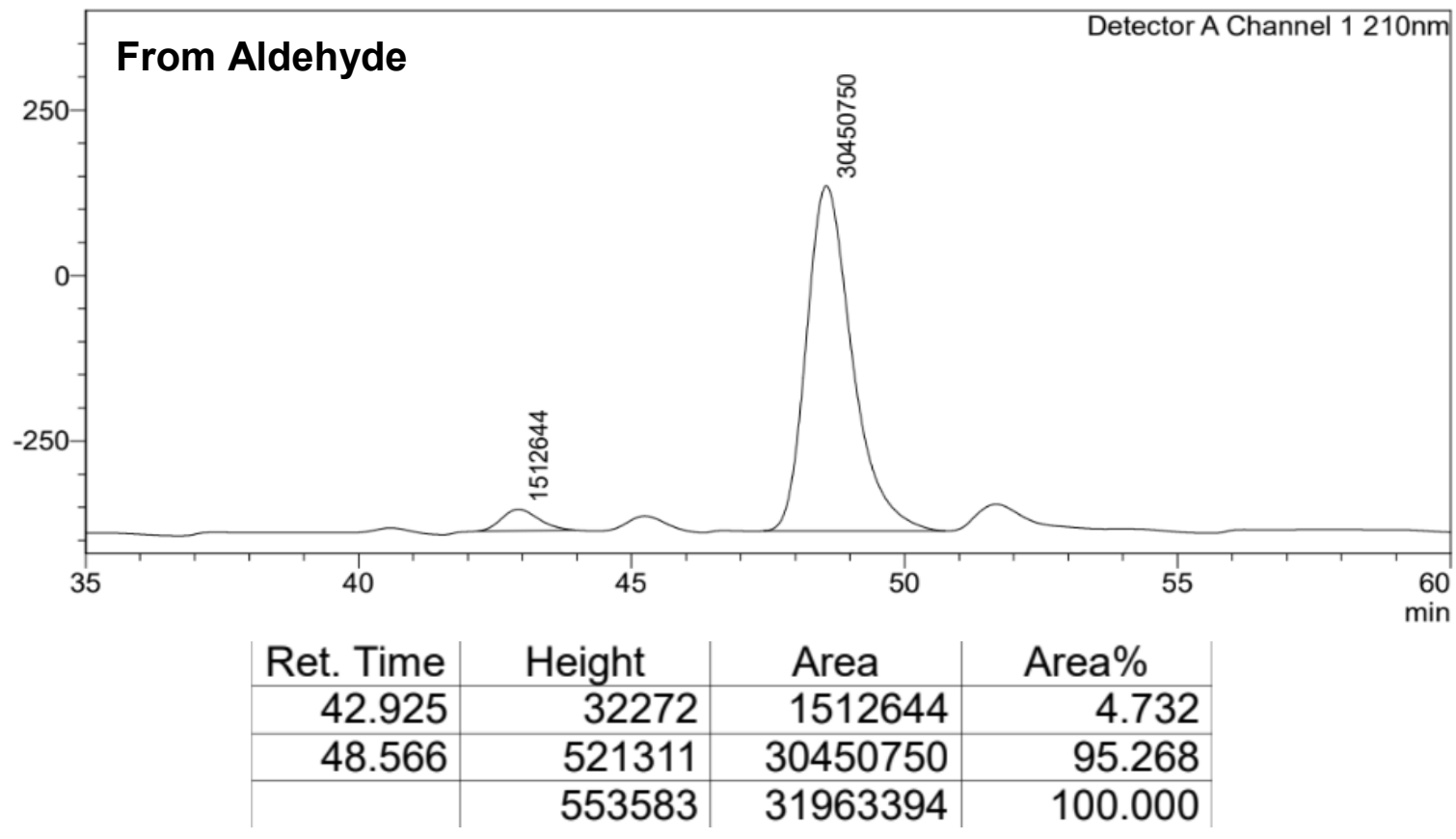




\section{(1S,2S)-2-(benzhydryloxy)-1-(2-fluoro-4-methoxyphenyl)-2-methylbut-3-en-1-ol (4f)}

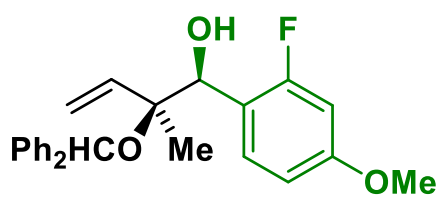

Alcohol oxidation level: Alcohol $2 \mathrm{f}(31.2 \mathrm{mg}, 0.2 \mathrm{mmol})$ was subjected to standard reaction conditions $\left(75^{\circ} \mathrm{C}, 24 \mathrm{~h}\right)$. Upon flash column chromatography $\left(\mathrm{SiO}_{2}, 5: 95 \mathrm{EtOAc}\right.$ :hexanes), the title compound $\mathbf{4 f}$ was obtained as a colorless oil in $76 \%$ yield $(59.6 \mathrm{mg}, 0.152 \mathrm{mmol}, 16: 1 \mathrm{dr}$, $94 \%$ ee).

Aldehyde oxidation level: Aldehyde $3 \mathrm{f}$ ( $30.8 \mathrm{mg}, 0.2 \mathrm{mmol}$ ) was subjected to standard reaction conditions $\left(75^{\circ} \mathrm{C}, 24 \mathrm{~h}\right)$. Upon flash column chromatography $\left(\mathrm{SiO}_{2}, 5: 95 \mathrm{EtOAc}\right.$ :hexanes), the title compound $\mathbf{4 f}$ was obtained as a colorless oil in $82 \%$ yield $(64.4 \mathrm{mg}, 0.164 \mathrm{mmol}, 17: 1 \mathrm{dr}, 95 \%$ ee).

$\operatorname{TLC}\left(\mathrm{SiO}_{2}\right) \mathrm{R}_{\mathrm{f}}=0.25$ (5:95 EtOAc:hexanes)

${ }^{1} \mathrm{H}$ NMR $\left(500 \mathrm{MHz}, \mathrm{CDCl}_{3}\right) \delta: 7.43(\mathrm{t}, J=8.5 \mathrm{~Hz}, 1 \mathrm{H}), 7.38-7.26(\mathrm{~m}, 5 \mathrm{H}), 7.25-7.16(\mathrm{~m}, 5 \mathrm{H}), 6.68$ (dd, $J=8.7,2.5 \mathrm{~Hz}, 1 \mathrm{H}$ ), 6.50 (dd, $J=12.1,2.5 \mathrm{~Hz}, 1 \mathrm{H}$ ), 5.93 (ddd, $J=17.7,10.9,2.2 \mathrm{~Hz}, 1 \mathrm{H}$ ), 5.52 (s, 1H), 5.20 (d, J=10.9 Hz, 1H), $5.06(\mathrm{~d}, J=17.6 \mathrm{~Hz}, 1 \mathrm{H}), 5.00(\mathrm{~d}, J=3.7 \mathrm{~Hz}, 1 \mathrm{H}), 3.75(\mathrm{~s}, 3 \mathrm{H}), 3.17$ $(\mathrm{d}, J=4.0 \mathrm{~Hz}, 1 \mathrm{H}), 1.06(\mathrm{~s}, 3 \mathrm{H})$.

${ }^{13} \mathrm{C}$ NMR $\left(125 \mathrm{MHz}, \mathrm{CDCl}_{3}\right) \delta: 161.1(\mathrm{~d}, J=202.6 \mathrm{~Hz}), 160.4,159.9,144.7,144.2,139.8$ (d, $J=1.4$ $\mathrm{Hz}), 129.9$ (d, $J=5.9 \mathrm{~Hz}), 128.6,128.5,128.5,127.7,127.2,126.9,126.7,126.7,118.8$ (d, $J=13.2$ $\mathrm{Hz}), 118.2,109.8(\mathrm{~d}, J=2.9 \mathrm{~Hz}), 100.9(\mathrm{~d}, J=26.6 \mathrm{~Hz}), 83.8,77.1,72.9,55.6,16.8$.

${ }^{19}$ F NMR (470 MHz, $\left.\mathrm{CDCl}_{3}\right) \delta:-112.80--113.34(\mathrm{~m})$.

HRMS $(\mathrm{Na}+, m / z)$ for $\mathrm{C}_{25} \mathrm{H}_{25} \mathrm{FO}_{3}$ : calcd. $=415.1680 ;$ found $=415.1689$.

FTIR (neat): 3567, 1626, 1508, 1453, 1285, 1112, 1025, $905 \mathrm{~cm}^{-1}$.

HPLC: (Chiralcel AD-H column, Hexane:2-PrOH = 98:02, $0.5 \mathrm{~mL} / \mathrm{min}, 230 \mathrm{~nm}$ )

$[\alpha]_{D}^{24}=-5.0^{\circ}\left(\mathrm{c}=0.4, \mathrm{CHCl}_{3}\right)$. 

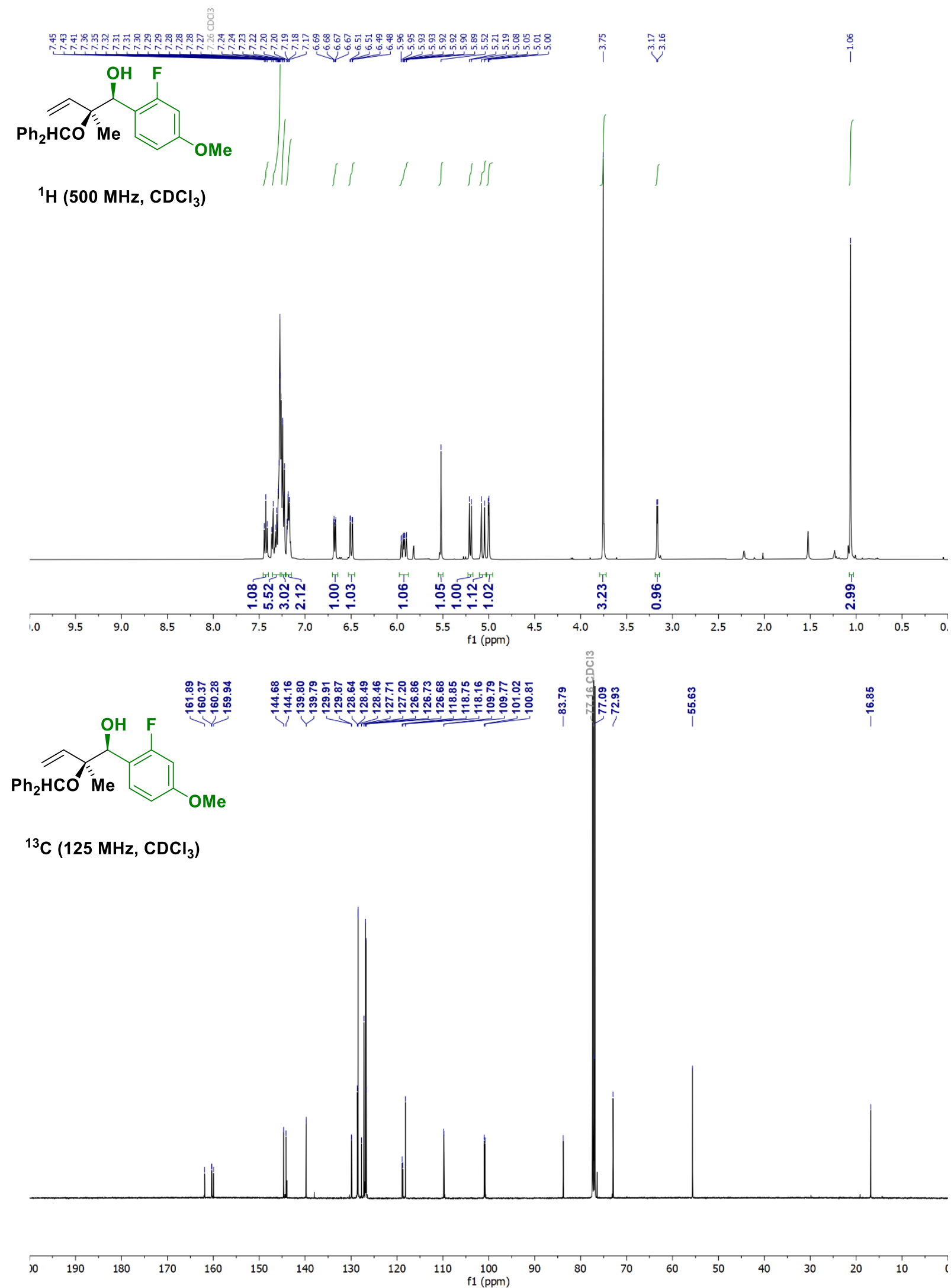


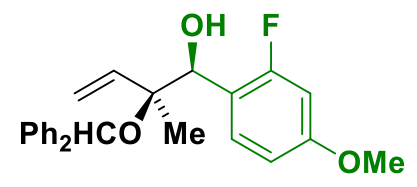

울용

兴

${ }^{19} \mathrm{~F}\left(470 \mathrm{MHz}, \mathrm{CDCl}_{3}\right)$

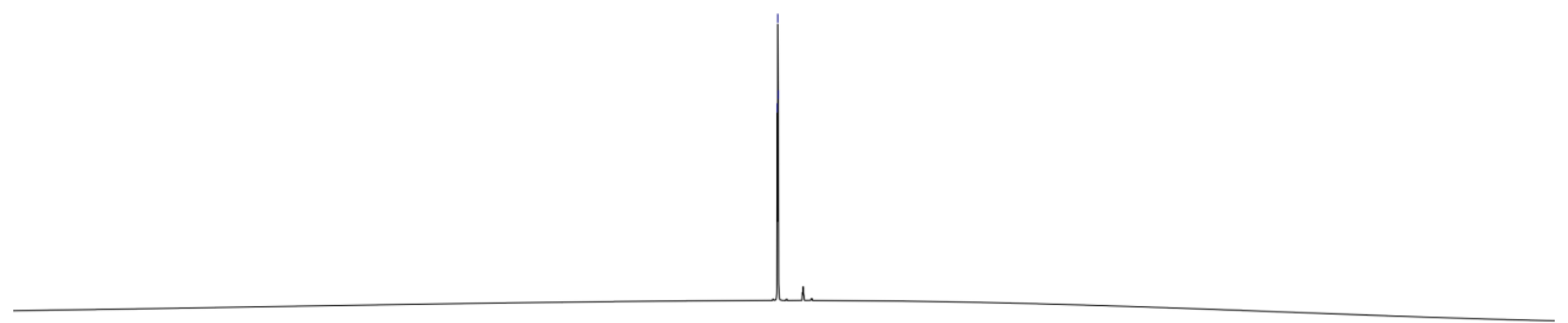



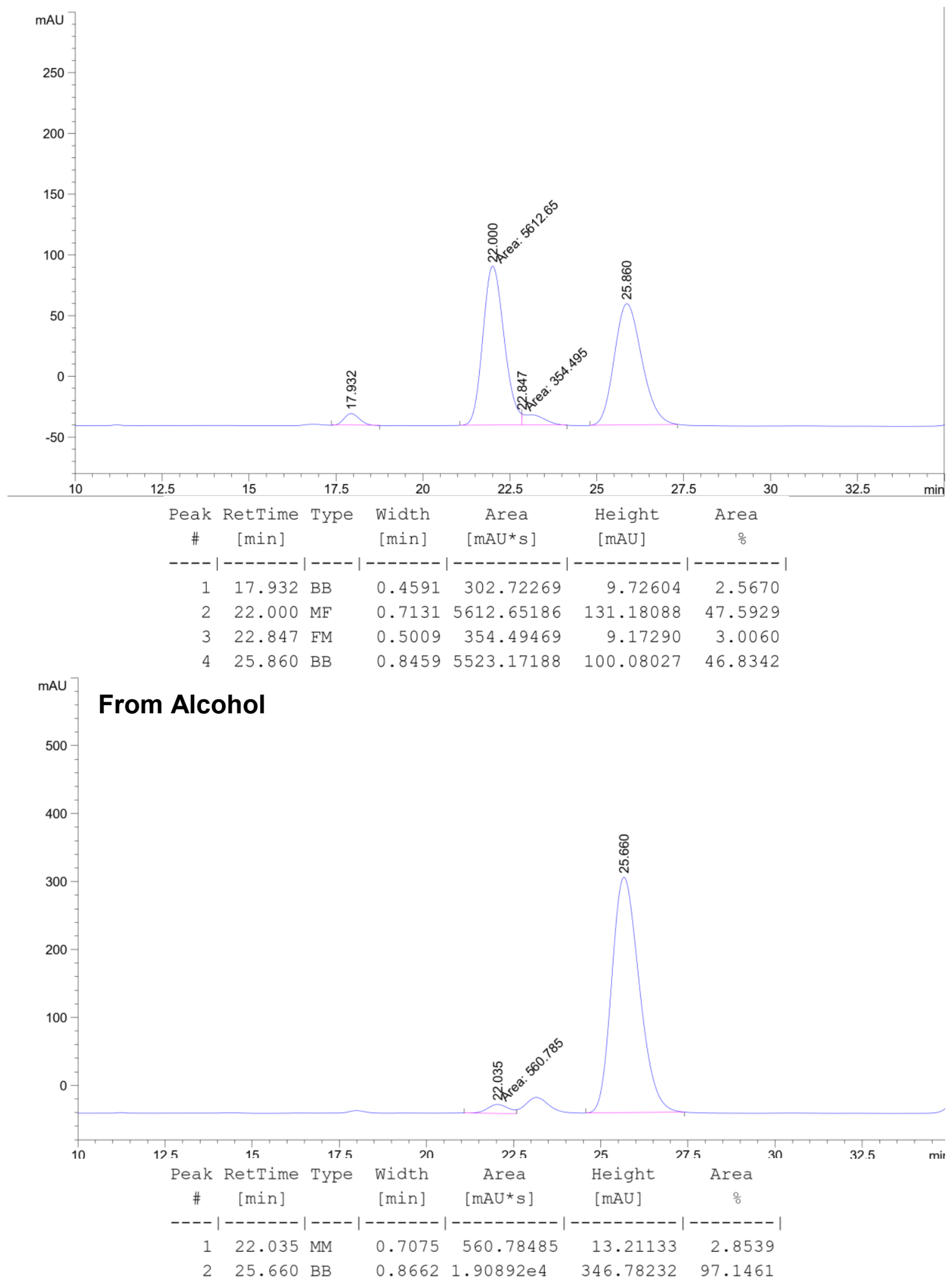


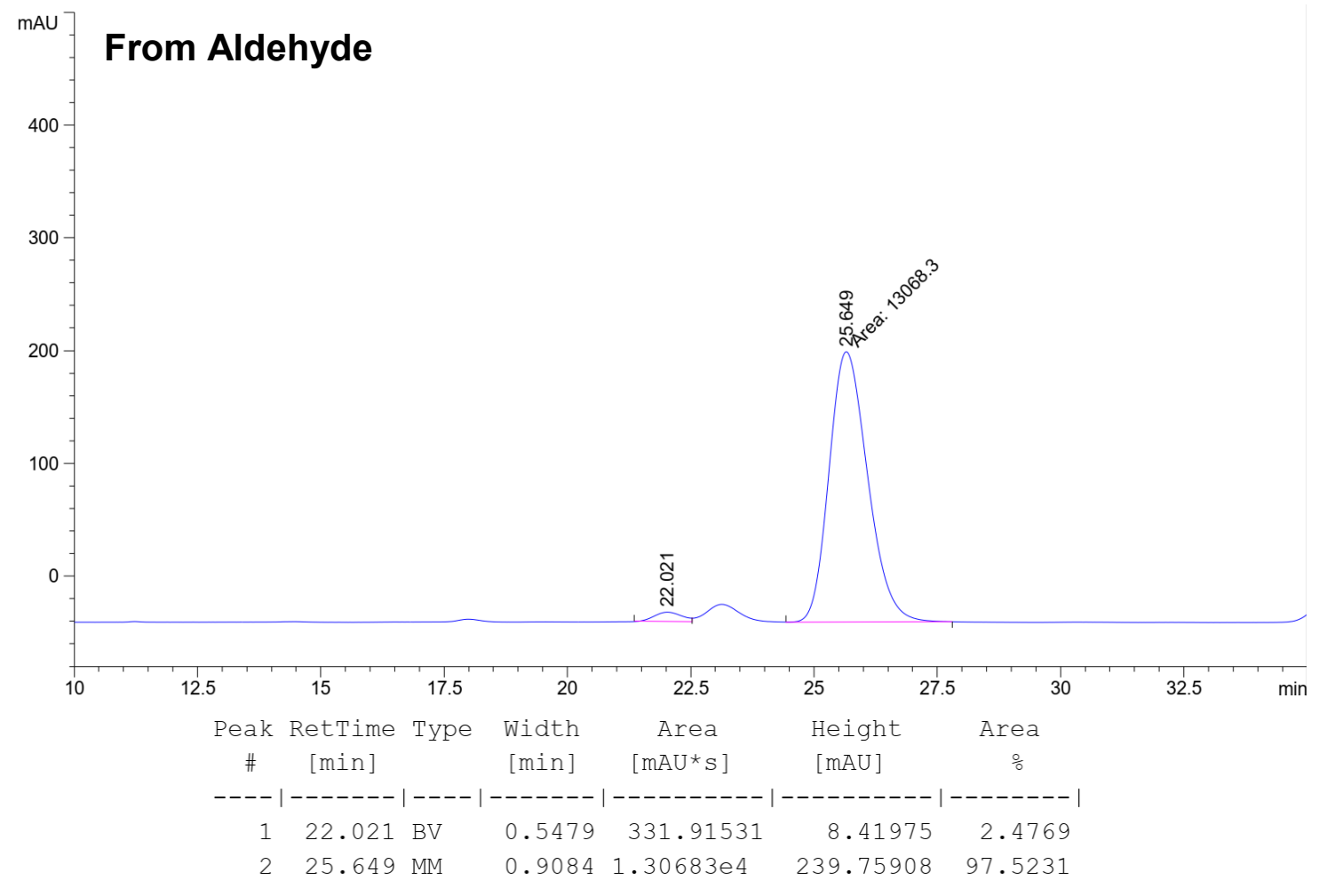


(1S,2S)-2-(benzhydryloxy)-1-(2-bromo-4,5-dimethoxyphenyl)-2-methylbut-3-en-1-ol (4g)<smiles>C=C[C@](C)(OCc1ccccc1)[C@H](O)c1cc(OC)c(OC)cc1Br</smiles>

Alcohol oxidation level: Alcohol $\mathbf{2 g}(49.4 \mathrm{mg}, 0.2 \mathrm{mmol})$ was subjected to standard reaction conditions (10 mol\% catalyst loading, $75^{\circ} \mathrm{C}, \mathbf{4 8} \mathrm{h}$ ). Upon flash column chromatography $\left(\mathrm{SiO}_{2}\right.$, 12:88 EtOAc:hexanes), the title compound $\mathbf{4 g}$ was obtained as a tan solid in $82 \%$ yield $(79.5 \mathrm{mg}$, $0.164 \mathrm{mmol}, 7: 1 \mathrm{dr}, 87 \%$ ee).

Aldehyde oxidation level: Aldehyde $3 \mathrm{~g}(49.0 \mathrm{mg}, 0.2 \mathrm{mmol})$ was subjected to standard reaction conditions $\left(75^{\circ} \mathrm{C}, 24 \mathrm{~h}\right)$. Upon flash column chromatography (12:88 EtOAc:hexanes), the title compound $\mathbf{4 g}$ was obtained as a tan solid in $84 \%$ yield $(81.2 \mathrm{mg}, 0.168 \mathrm{mmol}, 9: 1 \mathrm{dr}, 86 \%$ ee).

$\operatorname{TLC}\left(\mathrm{SiO}_{2}\right) \mathrm{R}_{\mathrm{f}}=0.1$ (10:90 EtOAc:hexanes)

${ }^{1} \mathrm{H}$ NMR $\left(400 \mathrm{MHz}, \mathrm{CDCl}_{3}\right) \delta: 7.31-7.18(\mathrm{~m}, 8 \mathrm{H}), 7.19-7.11(\mathrm{~m}, 3 \mathrm{H}), 6.89(\mathrm{~s}, 1 \mathrm{H}), 6.07(\mathrm{dd}, J=$ 17.6, $10.9 \mathrm{~Hz}, 1 \mathrm{H}), 5.51(\mathrm{~s}, 1 \mathrm{H}), 5.19(\mathrm{dd}, J=11.0,1.1 \mathrm{~Hz}, 1 \mathrm{H}), 5.12-5.07(\mathrm{~m}, 1 \mathrm{H}), 5.07-5.05(\mathrm{~m}$, $1 \mathrm{H}), 3.80(\mathrm{~s}, 3 \mathrm{H}), 3.77(\mathrm{~s}, 3 \mathrm{H}), 3.17(\mathrm{~d}, J=4.8 \mathrm{~Hz}, 1 \mathrm{H}), 1.09(\mathrm{~s}, 3 \mathrm{H})$.

${ }^{13} \mathrm{C} \mathrm{NMR}\left(100 \mathrm{MHz}, \mathrm{CDCl}_{3}\right)$ $\delta: 149.1,148.2,144.5,144.1,140.2,131.3,128.5,128.5,127.3,127.2$, $126.9,126.7,117.8,115.0,114.6,112.5,84.0,77.6,77.1,56.2,56.0,18.2$.

HRMS $(\mathrm{Na}+, m / z)$ for $\mathrm{C}_{26} \mathrm{H}_{27} \mathrm{BrO}_{4}$ : calcd. = 505.0985; found $=505.0970$.

FTIR (neat): 3519, 2974, 1605, 1498, 1435, 1257, 1153, 1021, 743, $703 \mathrm{~cm}^{-1}$

HPLC: (Two connected Chiralcel AD-H columns, Hexane:2-PrOH = 95:05, $1.0 \mathrm{~mL} / \mathrm{min}, 210 \mathrm{~nm}$ ) $[\alpha]_{D}^{24}=+12.5^{\circ}\left(\mathrm{c}=0.2, \mathrm{CHCl}_{3}\right)$ 

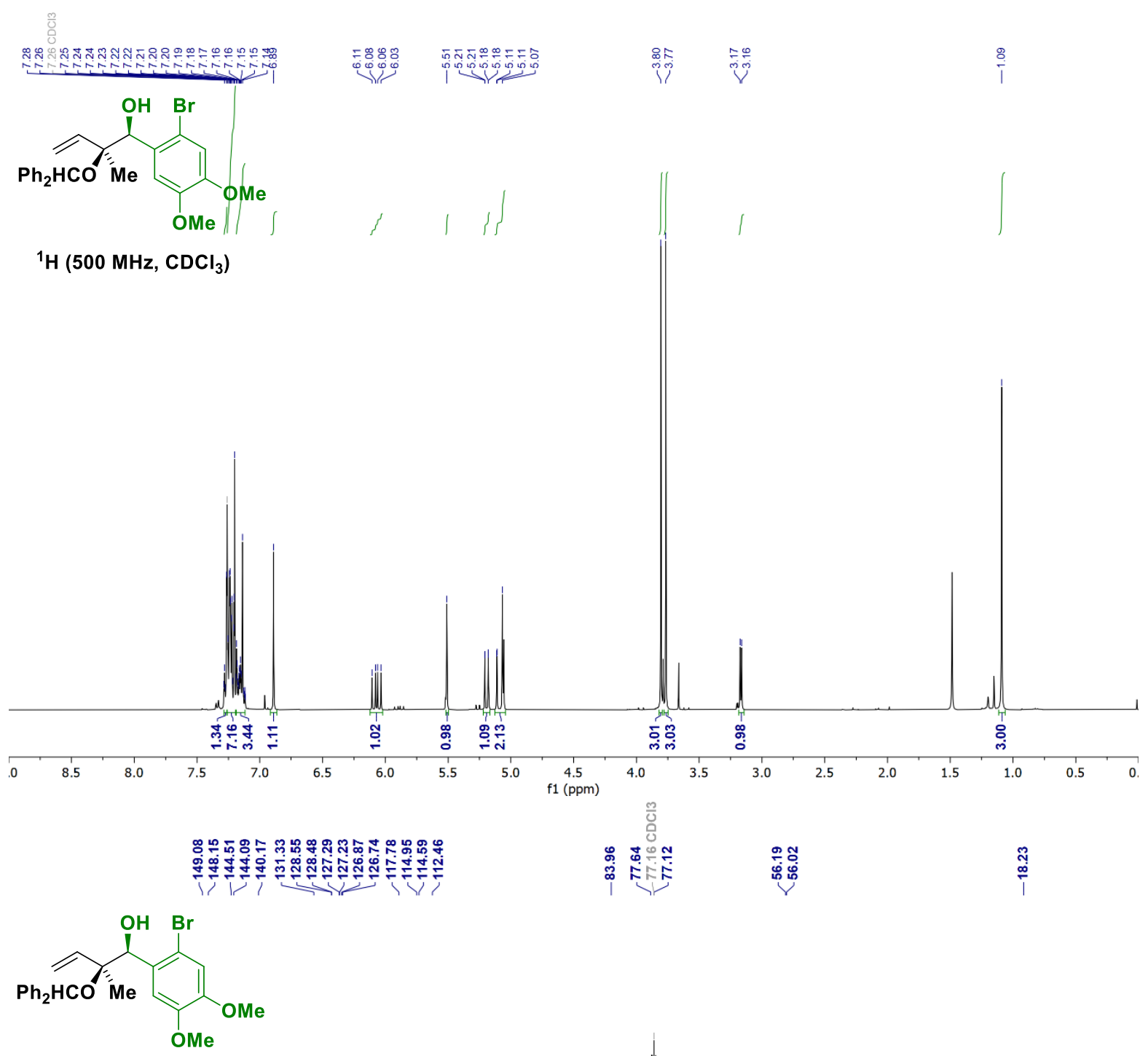

${ }^{13} \mathrm{C}\left(125 \mathrm{MHz}, \mathrm{CDCl}_{3}\right)$

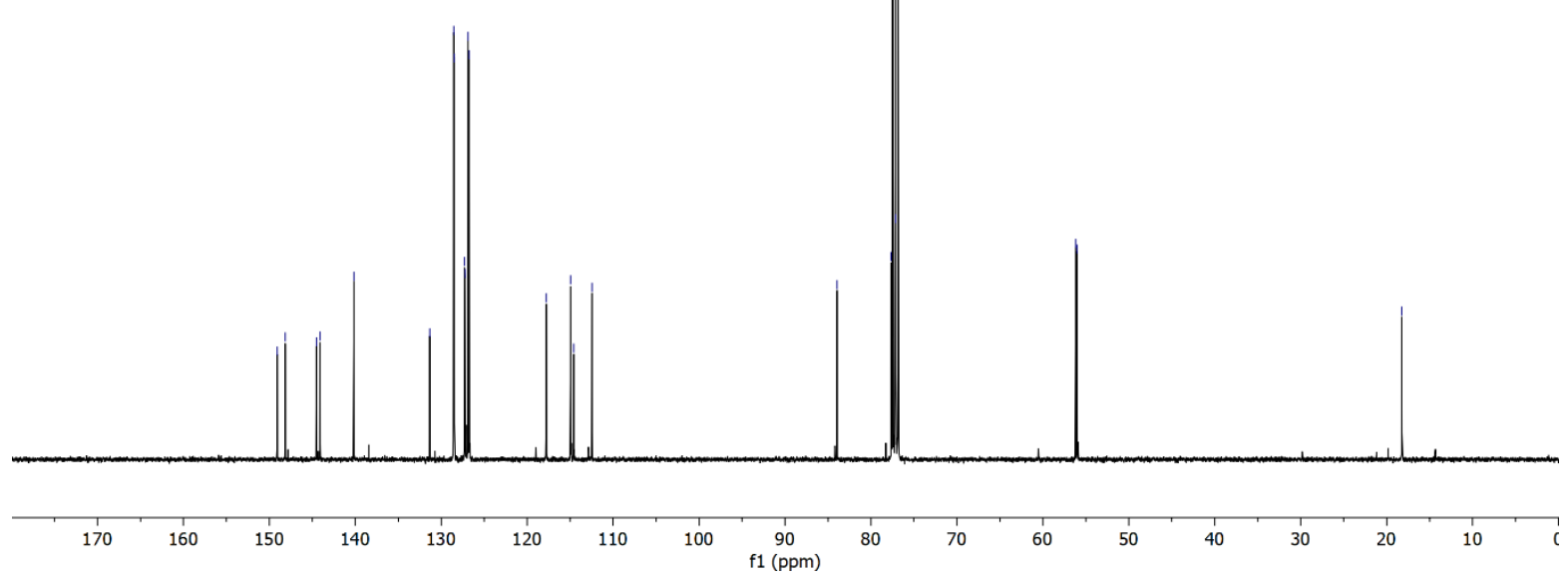



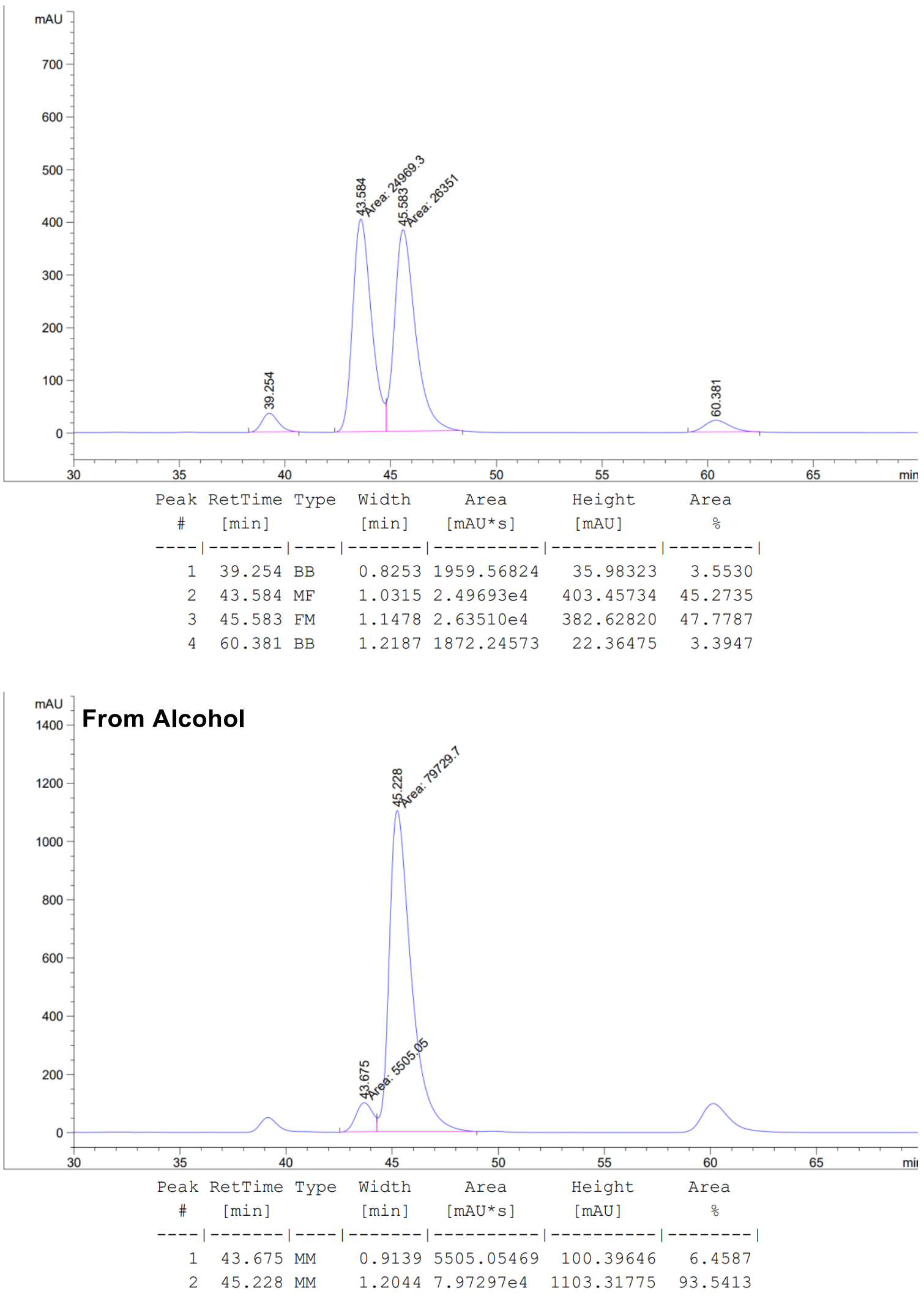


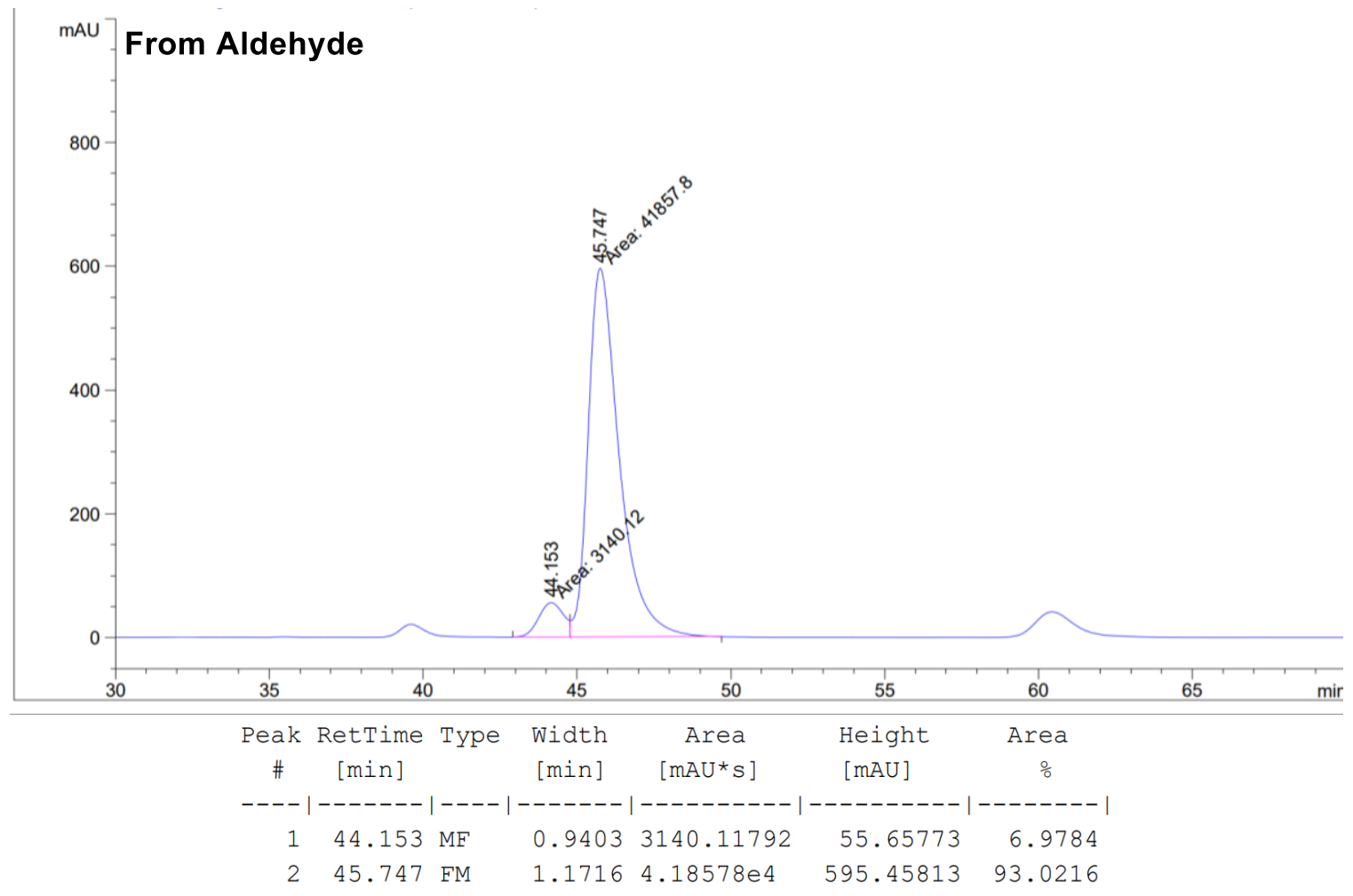


(1S,2S)-2-(benzhydryloxy)-1-(furan-3-yl)-2-methylbut-3-en1-ol (4h)

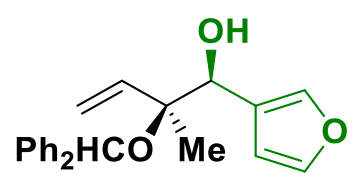

Alcohol oxidation level: Alcohol $\mathbf{2 h}(19.6 \mathrm{mg}, 0.2 \mathrm{mmol})$ was subjected to standard reaction conditions $\left(75^{\circ} \mathrm{C}, 24 \mathrm{~h}\right)$. Upon flash column chromatography $\left(\mathrm{SiO}_{2}, 5: 95\right.$ EtOAc:hexanes), the title compound $4 \mathrm{~h}$ was obtained as a yellow oil in $80 \%$ yield $(53.6 \mathrm{mg}, 0.16 \mathrm{mmol}, 17: 1 \mathrm{dr}, 96 \%$ ee).

Aldehyde oxidation level: Aldehyde $3 \mathrm{~h}(19.2 \mathrm{mg}, 0.2 \mathrm{mmol})$ was subjected to standard reaction conditions $\left(75^{\circ} \mathrm{C}, 24 \mathrm{~h}\right)$. Upon flash column chromatography $\left(\mathrm{SiO}_{2}, 5: 95\right.$ EtOAc:hexanes), the title compound $4 \mathrm{~h}$ was obtained as a yellow oil in $92 \%$ yield ( $61.5 \mathrm{mg}, 0.184 \mathrm{mmol}, 19: 1 \mathrm{dr}, 98 \%$ ee).

$\operatorname{TLC}\left(\mathrm{SiO}_{2}\right) \mathrm{R}_{\mathrm{f}}=0.25$ (10:90 EtOAc:hexanes)

${ }^{1} \mathrm{H}$ NMR $\left(500 \mathrm{MHz}, \mathrm{CDCl}_{3}\right)$ 8: $7.33-7.26(\mathrm{~m}, 3 \mathrm{H}), 7.25-7.18(\mathrm{~m}, 7 \mathrm{H}), 7.18-7.12(\mathrm{~m}, 2 \mathrm{H}), 6.32(\mathrm{~d}$, $J=1.8 \mathrm{~Hz}, 1 \mathrm{H}), 5.77(\mathrm{dd}, J=17.6,10.9 \mathrm{~Hz}, 1 \mathrm{H}), 5.51(\mathrm{~s}, 1 \mathrm{H}), 5.24(\mathrm{~d}, J=10.6 \mathrm{~Hz}, 1 \mathrm{H}), 5.13(\mathrm{~d}, J=$ $17.7 \mathrm{~Hz}, 1 \mathrm{H}), 4.63(\mathrm{~s}, 1 \mathrm{H}), 3.04(\mathrm{~s}, 1 \mathrm{H}), 1.04(\mathrm{~s}, 3 \mathrm{H})$.

${ }^{13} \mathrm{C}$ NMR (125 MHz, $\left.\mathrm{CDCl}_{3}\right)$ 8: 144.6, 144.1, 142.4, 140.6, 139.9, 128.6, 128.4, 127.3, 127.2, 126.9, $126.8,124.1,118.9,110.1,83.4,77.1,73.9,16.6$.

HRMS $(\mathrm{Na}+, m / z)$ for $\mathrm{C}_{22} \mathrm{H}_{22} \mathrm{O}_{3}$ : calcd. = 537.1461; found $=357.1452$.

FTIR (neat): 3547, 3026, 1598, 1493, 1452, 1021, 874, 740, $698 \mathrm{~cm}^{-1}$

HPLC: (Chiralcel AD-H column, Hexane:2-PrOH = 98:02, $0.5 \mathrm{~mL} / \mathrm{min}, 210 \mathrm{~nm}$ )

$[\alpha]_{D}^{24}=-12.5^{\circ}\left(\mathrm{c}=0.2, \mathrm{CHCl}_{3}\right)$ 


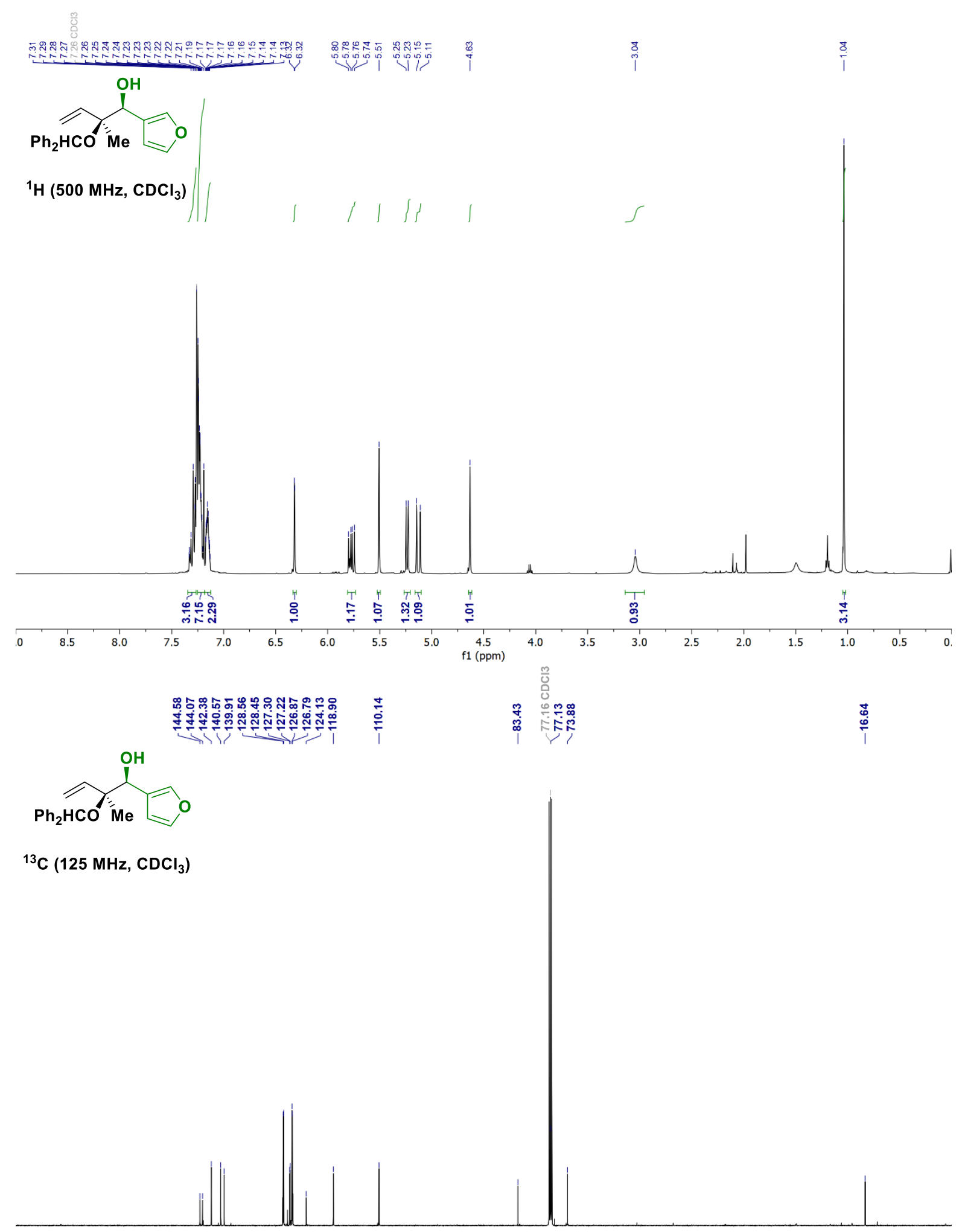

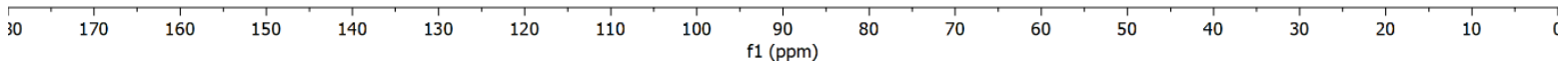



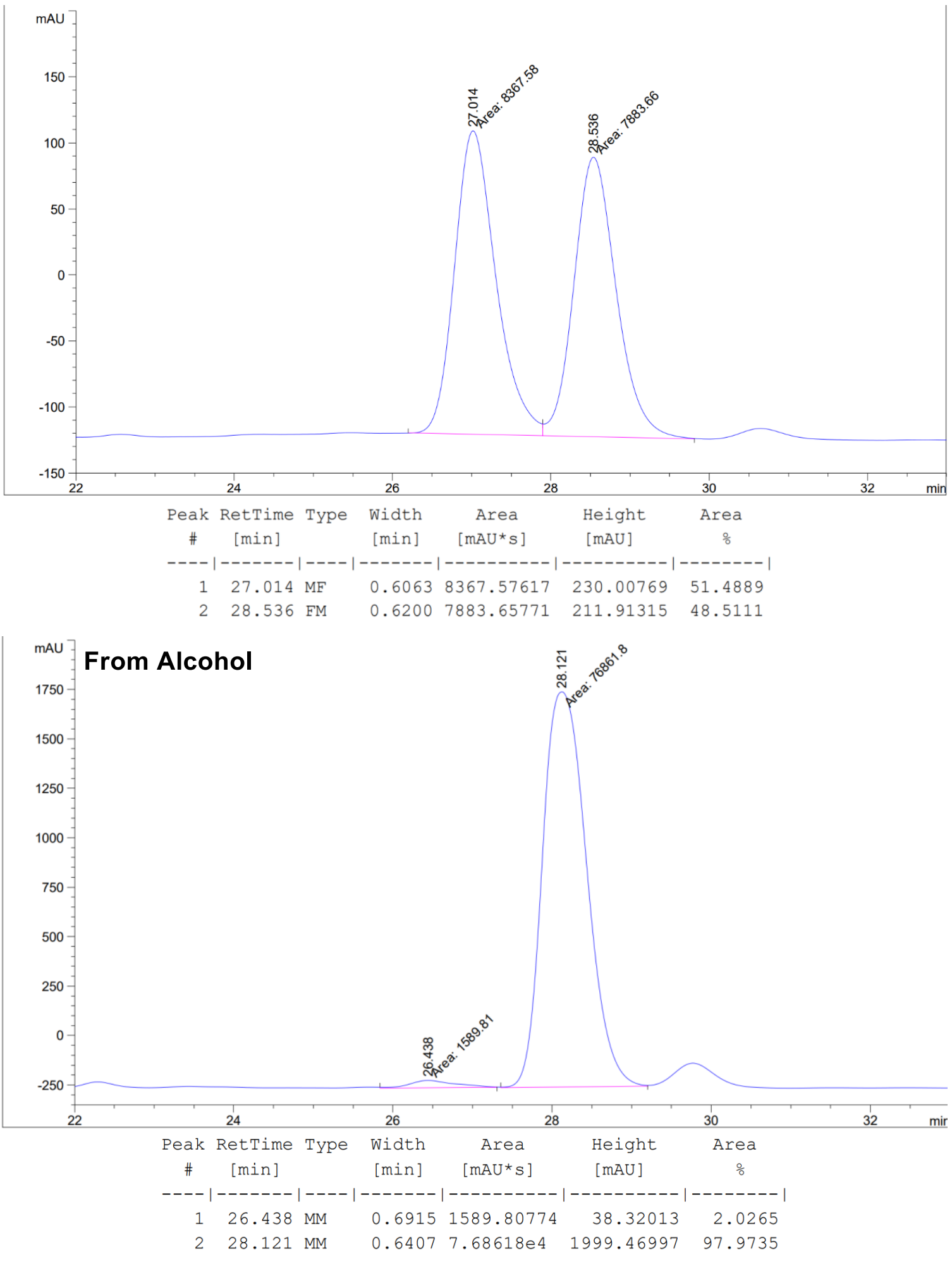


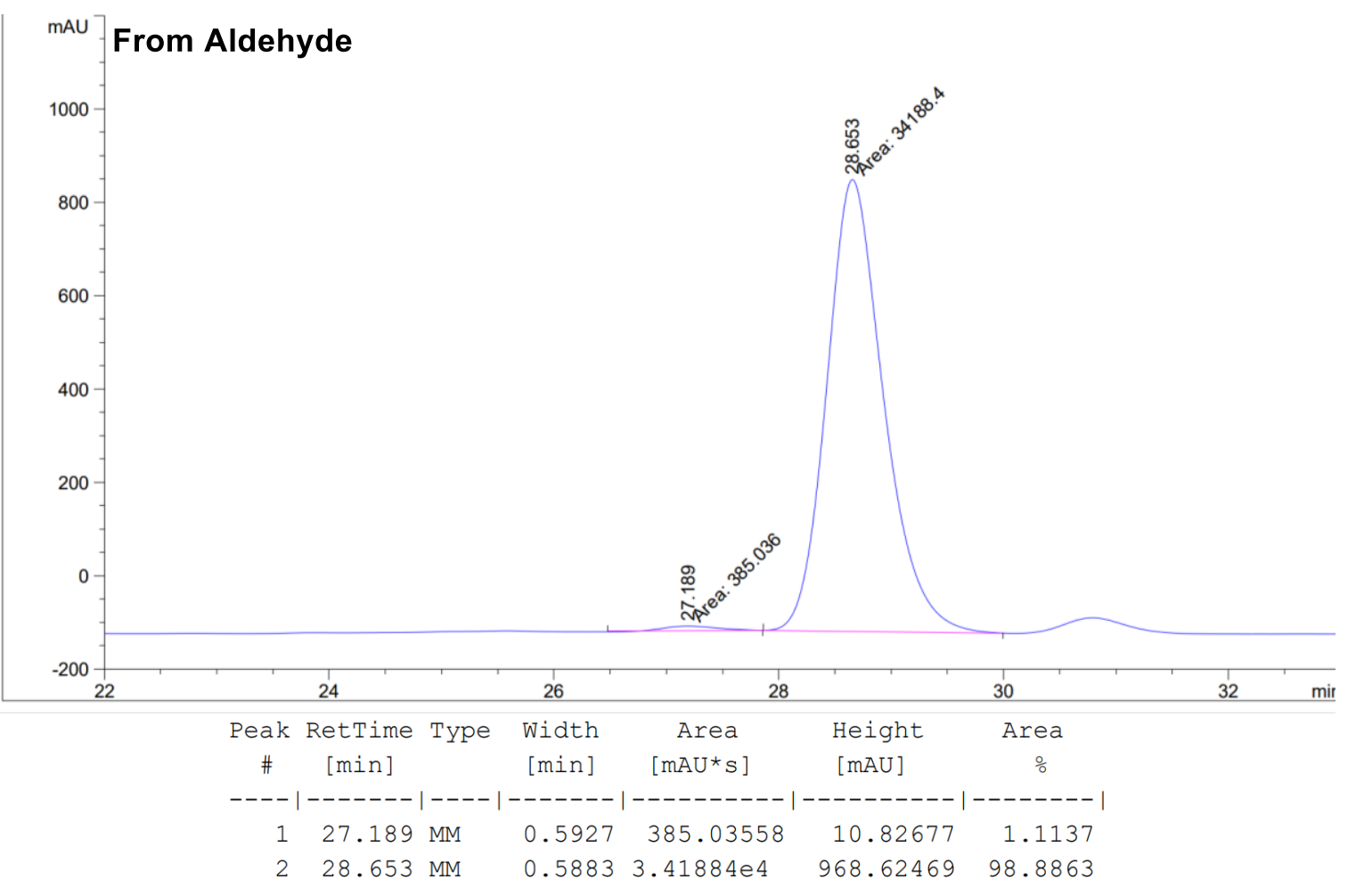


(1S,2S)-2-(benzhydryloxy)-1-(5-(3-chloro-4-methoxyphenyl)furan-2-yl)-2-methylbut-3-en-1-ol (4i)<smiles>C=C[C@](C)(OC(C)[PH2+])[C@@H](O)c1ccc(-c2ccc(OC)c(Cl)c2)o1</smiles>

Alcohol oxidation level: Aldehyde $2 \mathbf{i}(47.7 \mathrm{mg}, 0.2 \mathrm{mmol})$ was subjected to standard reaction conditions $\left(75^{\circ} \mathrm{C}, 24 \mathrm{~h}\right)$. Upon flash column chromatography $\left(\mathrm{SiO}_{2}, 10: 90\right.$ EtOAc:hexanes), the title compound $4 \mathbf{i}$ was obtained as an orange solid in $85 \%$ yield $(80.9 \mathrm{mg}, 0.17 \mathrm{mmol}, 4: 1 \mathrm{dr}, 90 \%$ ee).

Aldehyde oxidation level: Aldehyde $3 \mathbf{i}(47.3 \mathrm{mg}, 0.2 \mathrm{mmol})$ was subjected to standard reaction conditions $\left(75^{\circ} \mathrm{C}, 24 \mathrm{~h}\right)$. Upon flash column chromatography $\left(\mathrm{SiO}_{2}, 10: 90\right.$ EtOAc:hexanes), the title compound $4 \mathbf{i}$ was obtained as an orange solid in $82 \%$ yield $(78.1 \mathrm{mg}, 0.165 \mathrm{mmol}, 8: 1 \mathrm{dr}$, $91 \%$ ee).

$\operatorname{TLC}\left(\mathrm{SiO}_{2}\right) \mathrm{Rf}_{\mathrm{f}}=0.1$ (10:90 EtOAc:hexanes)

${ }^{1} \mathrm{H}$ NMR $\left(500 \mathrm{MHz}, \mathrm{CDCl}_{3}\right) \delta: 7.64(\mathrm{~d}, J=2.2 \mathrm{~Hz}, 1 \mathrm{H}), 7.48(\mathrm{dd}, J=8.6,2.2 \mathrm{~Hz}, 1 \mathrm{H}), 7.37-7.23(\mathrm{~m}$, $7 \mathrm{H}), 7.22-7.17(\mathrm{~m}, 2 \mathrm{H}), 6.92(\mathrm{~d}, J=8.6 \mathrm{~Hz}, 1 \mathrm{H}), 6.49(\mathrm{~d}, J=3.3 \mathrm{~Hz}, 1 \mathrm{H}), 6.36(\mathrm{~d}, J=3.3 \mathrm{~Hz}, 1 \mathrm{H})$, $5.96(\mathrm{dd}, J=17.6,10.9 \mathrm{~Hz}, 1 \mathrm{H}), 5.57(\mathrm{~s}, 1 \mathrm{H}), 5.31(\mathrm{dd}, J=11.0,1.0 \mathrm{~Hz}, 1 \mathrm{H}), 5.24(\mathrm{dd}, J=17.7,1.1$ $\mathrm{Hz}, 1 \mathrm{H}), 4.72(\mathrm{~d}, J=4.1 \mathrm{~Hz}, 1 \mathrm{H}), 3.92(\mathrm{~s}, 3 \mathrm{H}), 3.10(\mathrm{~d}, J=5.1 \mathrm{~Hz}, 1 \mathrm{H}), 1.24(\mathrm{~s}, 3 \mathrm{H})$.

${ }^{13}$ C NMR (125 MHz, DMSO) $\delta: 155.1,153.5,150.2,145.6,145.0,139.5,128.0,127.9,126.6,126.4$, $126.2,126.0,124.6,124.5,123.1,121.5,116.7,113.2,109.8,105.6,81.7,75.5,73.6,56.2,19.2$.

HRMS $(\mathrm{Na}+, \mathrm{m} / \mathrm{z})$ for $\mathrm{C}_{29} \mathrm{H}_{27} \mathrm{ClO}_{4}$ : calcd. $=497.1490 ;$ found $=497.1479$.

FTIR (neat): 2923, 1594, 1490, 1267, 1062, 1019, $701 \mathrm{~cm}^{-1}$

HPLC: (Two connected Chiralcel AD-H columns, Hexane:2-PrOH = 95:05, $0.5 \mathrm{~mL} / \mathrm{min}, 210 \mathrm{~nm}$ ) $[\alpha]_{D}^{24}=-4.5^{\circ}\left(\mathrm{c}=1.0, \mathrm{CHCl}_{3}\right)$

MP: $[49-54]^{\circ} \mathrm{C}$ 


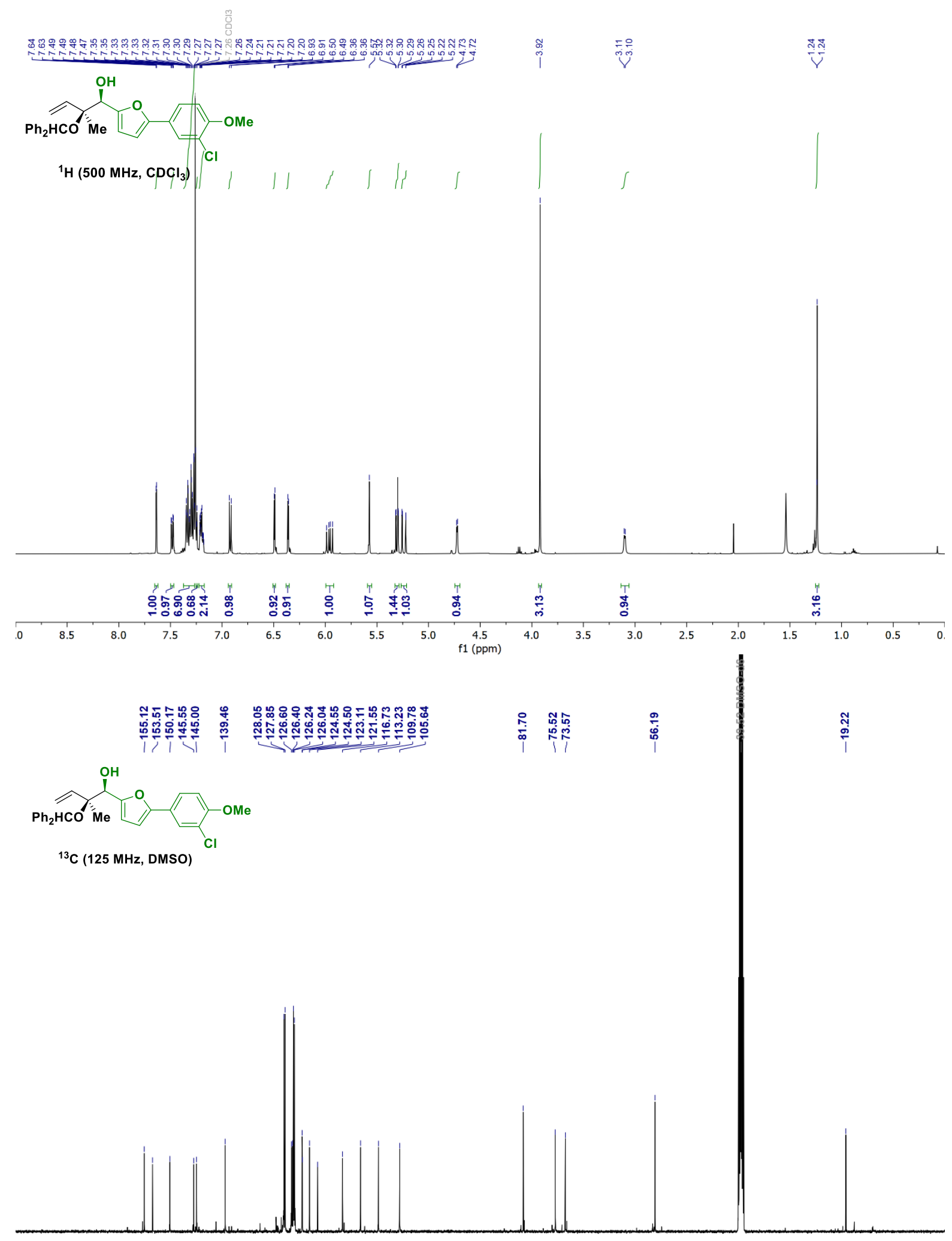

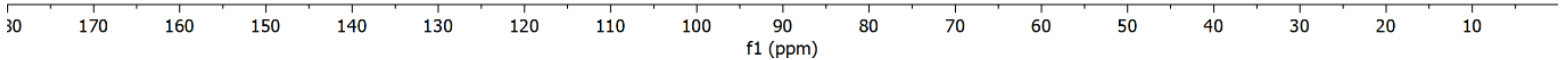


$\mathrm{mV}$

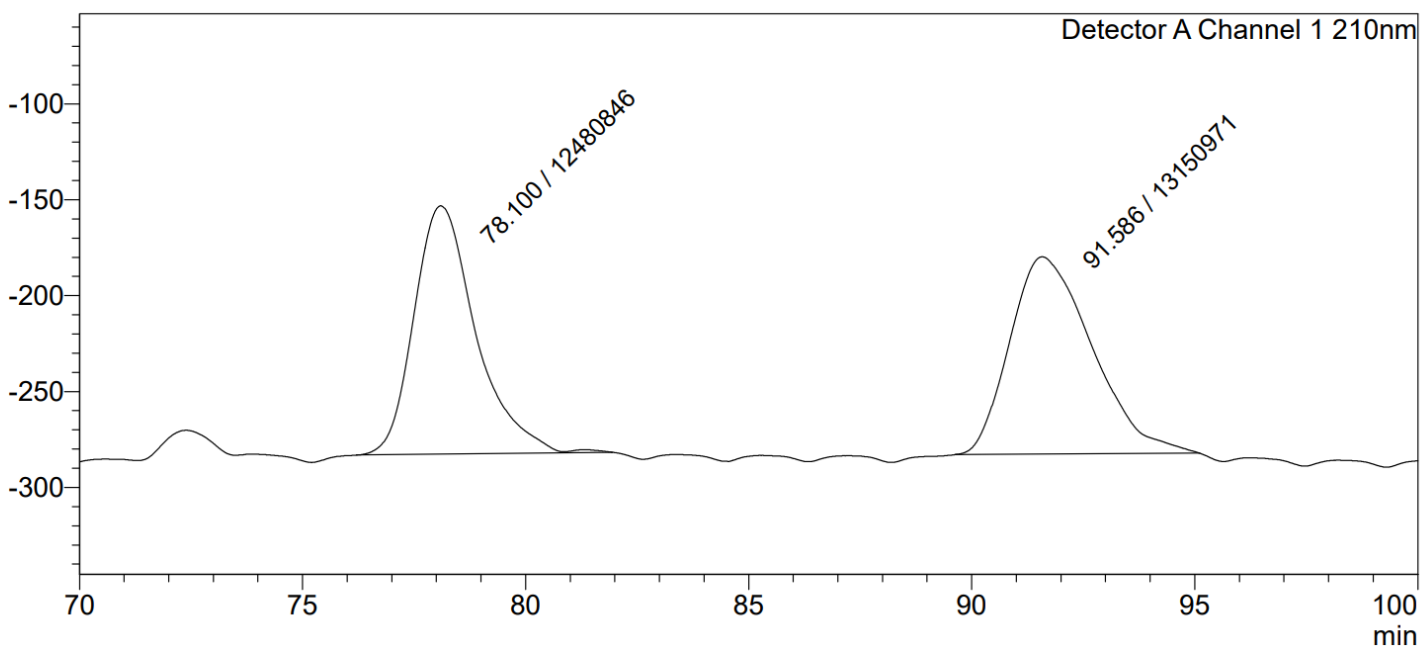

\begin{tabular}{|r|r|c|r|}
\hline Peak\# & Ret. Time & Area & Area $\%$ \\
\hline 1 & 78.100 & 12480846 & 48.693 \\
\hline 2 & 91.586 & 13150971 & 51.307 \\
\hline Total & & 25631816 & 100.000 \\
\hline
\end{tabular}

$\mathrm{mV}$

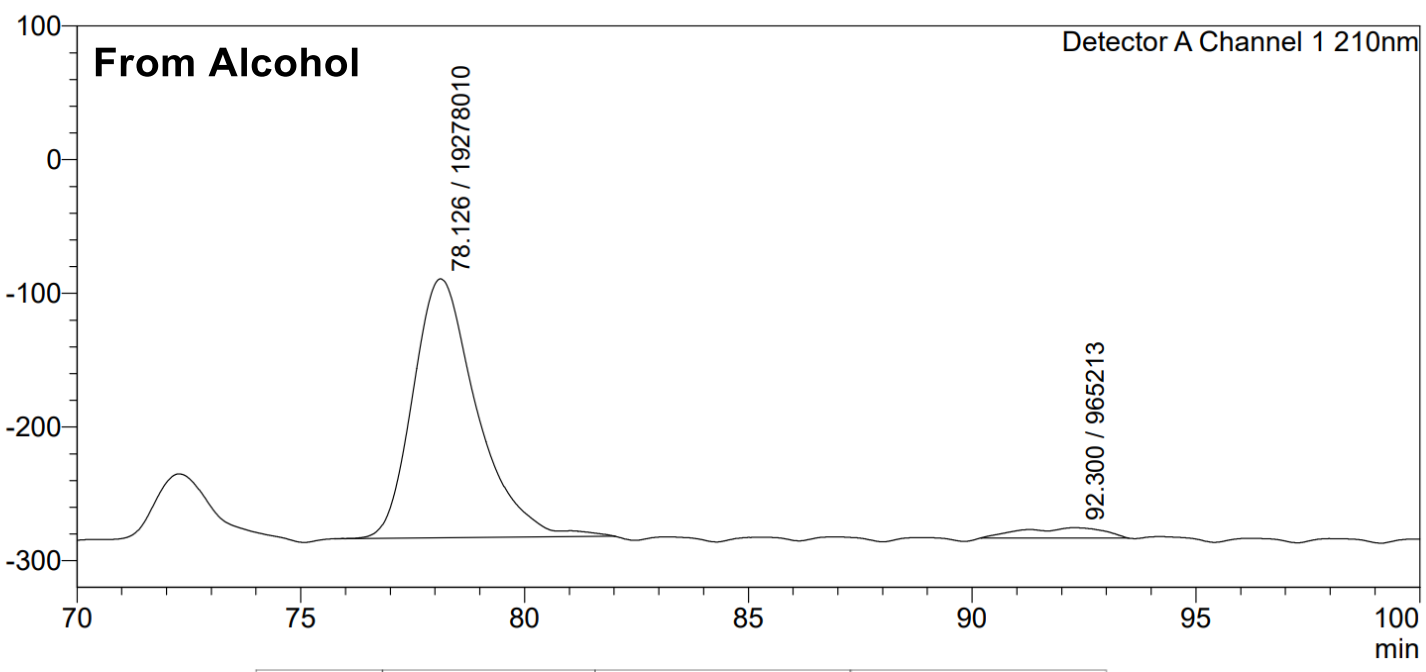

\begin{tabular}{|r|r|r|r|}
\hline Peak\# & Ret. Time & \multicolumn{1}{|c|}{ Area } & Area\% \\
\hline 1 & 78.126 & 19278010 & 95.232 \\
\hline 2 & 92.300 & 965213 & 4.768 \\
\hline Total & & 20243222 & 100.000 \\
\hline
\end{tabular}




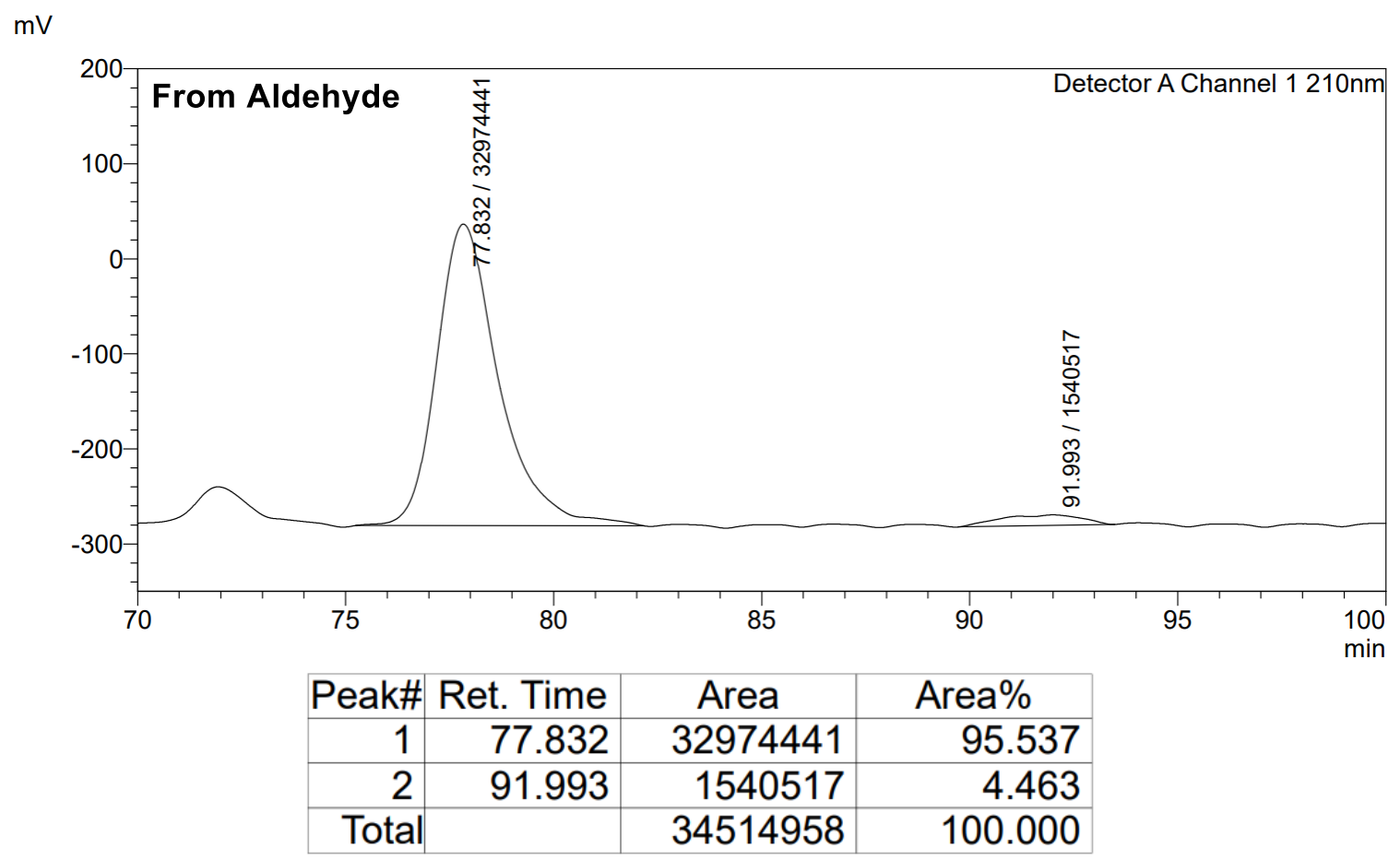


(1R,2S)-2-(benzhydryloxy)-2-methyl-1-(thiophen-2-yl)but-3-en-1-ol (4j)

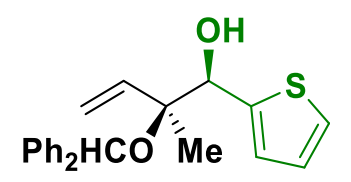

Alcohol oxidation level: Alcohol $\mathbf{2 j} \mathbf{j} 22.8 \mathrm{mg}, 0.2 \mathrm{mmol})$ was subjected to standard reaction conditions $\left(75^{\circ} \mathrm{C}, 24 \mathrm{~h}\right)$. Upon flash column chromatography $\left(\mathrm{SiO}_{2}, 5: 95\right.$ EtOAc:hexanes), the title compound $\mathbf{4} \mathbf{j}$ was obtained as a yellow solid in $76 \%$ yield $(53.6 \mathrm{mg}, 0.152 \mathrm{mmol}, 10: 1 \mathrm{dr}, 96 \%$ ee).

Aldehyde oxidation level: Aldehyde $3 \mathbf{j}(22.4 \mathrm{mg}, 0.2 \mathrm{mmol})$ was subjected to standard reaction conditions $\left(75^{\circ} \mathrm{C}, 24 \mathrm{~h}\right)$. Upon flash column chromatography $\left(\mathrm{SiO}_{2}, 5: 95\right.$ EtOAc:hexanes), the title compound $\mathbf{4} \mathbf{j}$ was obtained as a yellow solid in $83 \%$ yield $(58.4 \mathrm{mg}, 0.166 \mathrm{mmol}, 19: 1 \mathrm{dr}$, 98\% ee).

$\operatorname{TLC}\left(\mathrm{SiO}_{2}\right) \mathrm{R}_{\mathrm{f}}=0.3$ (10:90 EtOAc:hexanes)

${ }^{1}$ H NMR (400 MHz, DMSO) $\delta: 7.46-7.41(\mathrm{~m}, 2 \mathrm{H}), 7.39-7.36(\mathrm{~m}, 1 \mathrm{H}), 7.30-7.25(\mathrm{~m}, 4 \mathrm{H}), 7.25-$ $7.09(\mathrm{~m}, 6 \mathrm{H}), 5.68(\mathrm{dd}, J=17.6,10.9 \mathrm{~Hz}, 1 \mathrm{H}), 5.52(\mathrm{~s}, 1 \mathrm{H}), 5.35(\mathrm{~d}, J=4.9 \mathrm{~Hz}, 1 \mathrm{H}), 5.12(\mathrm{dd}, J=$ 10.9, $1.5 \mathrm{~Hz}, 1 \mathrm{H}), 5.06$ (dd, J = 17.6, $1.6 \mathrm{~Hz}, 1 \mathrm{H}), 4.67$ (d, J = 4.9 Hz, 1H), $1.08(\mathrm{~s}, 3 \mathrm{H})$.

${ }^{13} \mathrm{C}$ NMR (100 MHz, DMSO) $\delta: ~ 145.7,145.0,143.4,140.0,128.3,128.0,127.9,126.6,126.4,126.3$, $126.2,123.9,122.1,116.7,82.2,75.8,75.7,18.8$.

HRMS $(\mathrm{Na}+, m / z)$ for $\mathrm{C}_{22} \mathrm{H}_{22} \mathrm{O}_{2} \mathrm{~S}$ : calcd. $=373.1222 ;$ found $=373.1222$.

FTIR (neat): 3564, 2983, 1454, 1253, 1105, 1024, $747 \mathrm{~cm}^{-1}$

HPLC: (Phenomenex column Amylose 1, Hexane:2-PrOH = 99:01, $1.0 \mathrm{~mL} / \mathrm{min}, 210 \mathrm{~nm}$ ) $[\alpha]_{D}^{24}=+2.5^{\circ}\left(\mathrm{c}=0.2, \mathrm{CHCl}_{3}\right)$

MP: $[75-80]{ }^{\circ} \mathrm{C}$ 

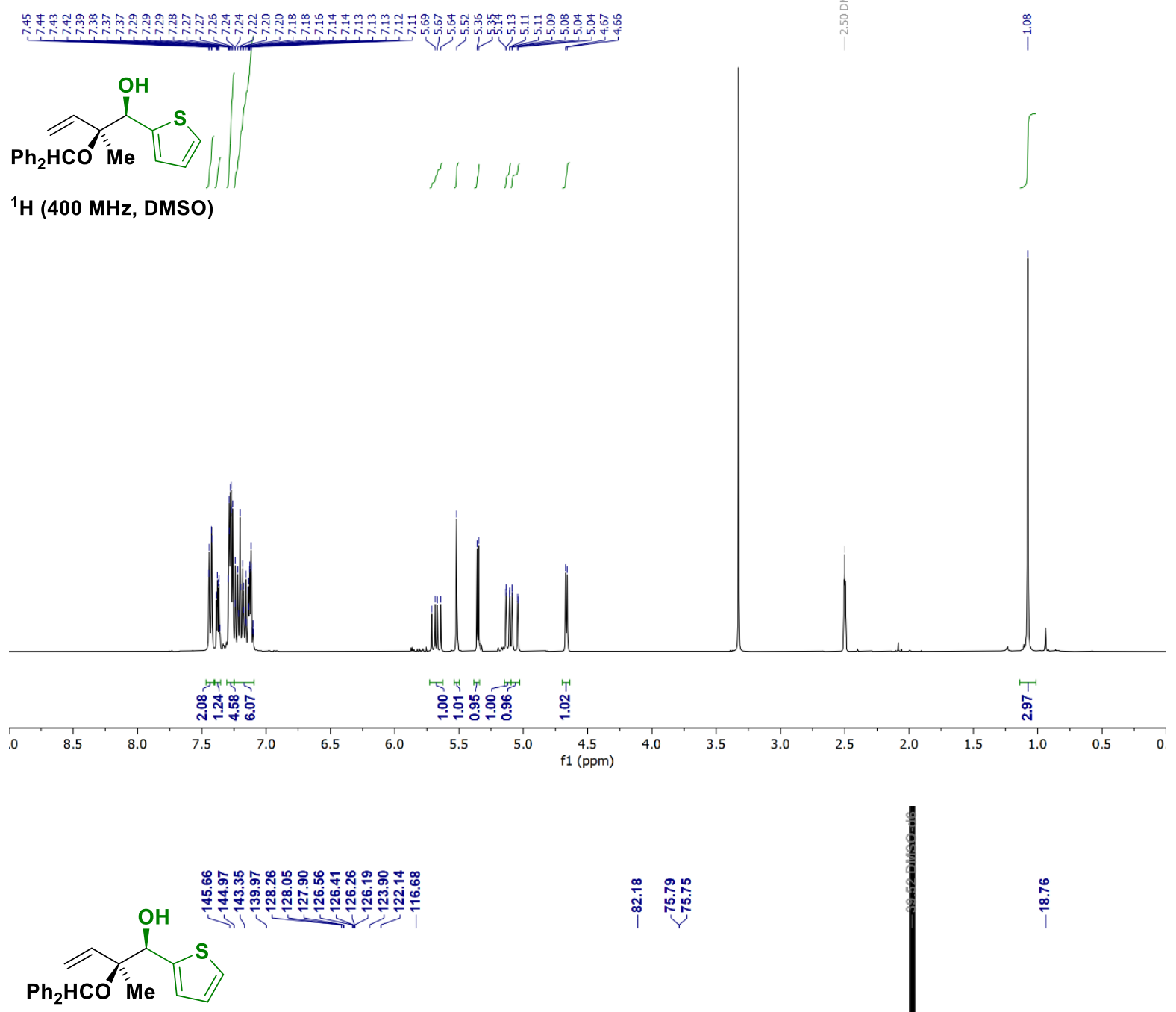

${ }^{13} \mathrm{C}$ (100 MHz, DMSO)
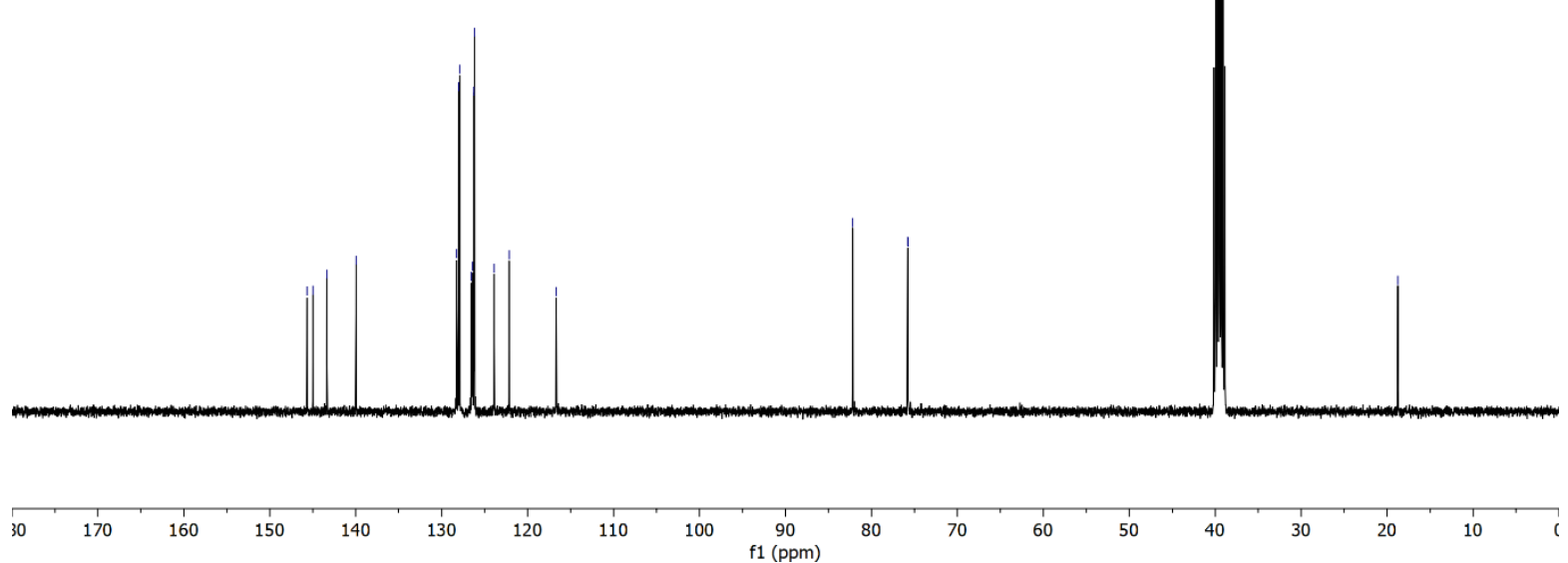

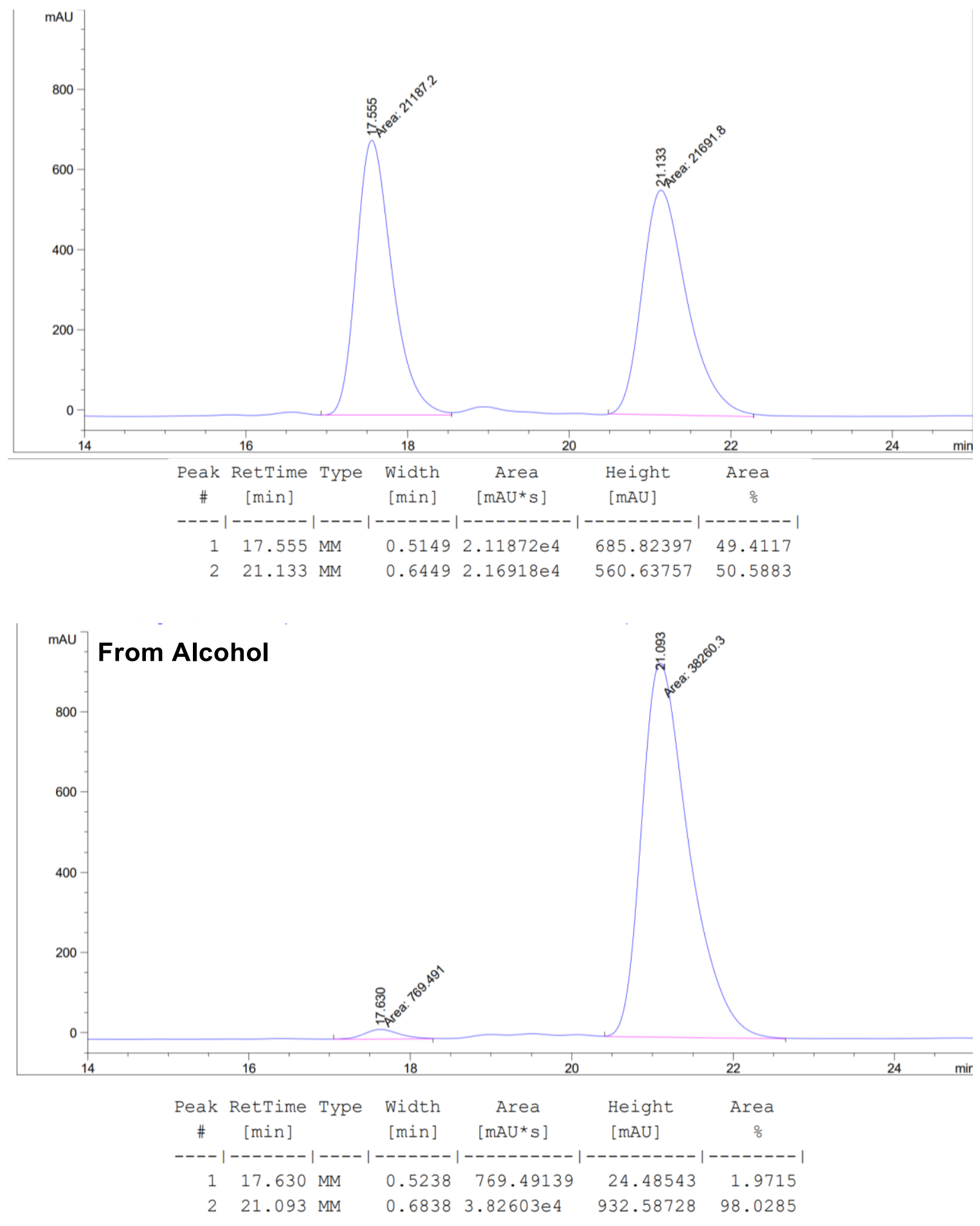


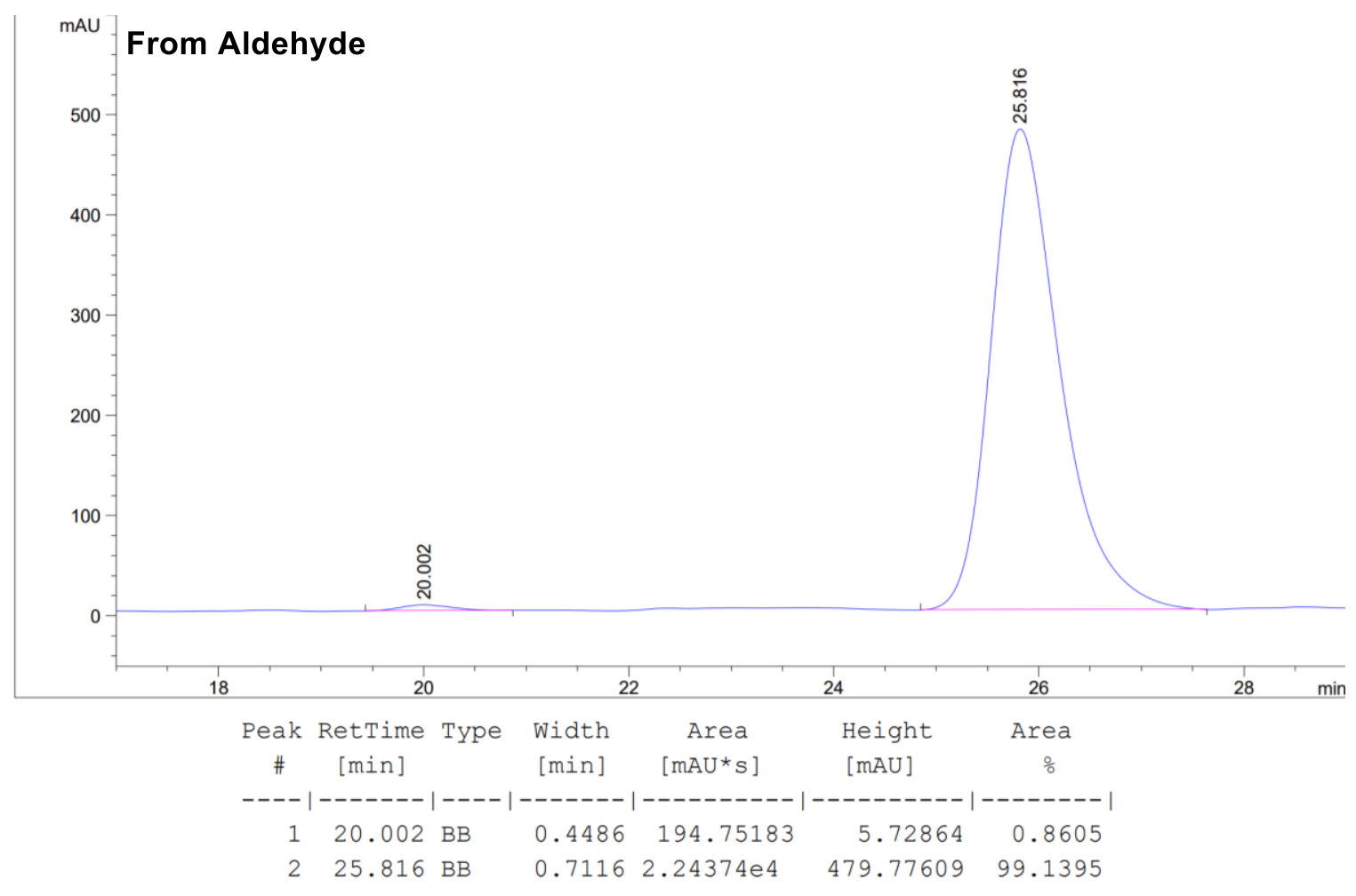


(1S,2S)-2-(benzhydryloxy)-1-(benzo[b]thiophen-3-yl)-2-methylbut-3-en-1-ol (4k)

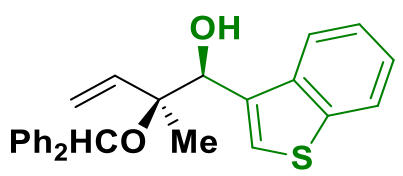

Alcohol oxidation level: Alcohol $\mathbf{2 k}(32.8 \mathrm{mg}, 0.2 \mathrm{mmol})$ was subjected to standard reaction conditions $\left(75^{\circ} \mathrm{C}, 24 \mathrm{~h}\right)$. Upon flash column chromatography $\left(\mathrm{SiO}_{2}, 6: 94\right.$ EtOAc:hexanes), the title compound $\mathbf{4 k}$ was obtained as an off-white solid in $76 \%$ yield $(60.9 \mathrm{mg}, 0.152 \mathrm{mmol}, 10: 1 \mathrm{dr}, 94 \%$ ee).

Aldehyde oxidation level: Aldehyde $3 \mathbf{k}(32.4 \mathrm{mg}, 0.2 \mathrm{mmol}$ ) was subjected to standard reaction conditions $\left(75^{\circ} \mathrm{C}, 24 \mathrm{~h}\right)$. Upon flash column chromatography $\left(\mathrm{SiO}_{2}, 6: 94\right.$ EtOAc:hexanes), the title compound $\mathbf{4 k}$ was obtained as an off-white solid in $74 \%$ yield $(59.5 \mathrm{mg}, 0.148 \mathrm{mmol}, 17: 1 \mathrm{dr}, 97 \%$ ee).

$\operatorname{TLC}\left(\mathrm{SiO}_{2}\right) \mathrm{R}_{\mathrm{f}}=0.6(20: 80$ EtOAc:hexanes)

${ }^{1} \mathbf{H}$ NMR $\left(500 \mathrm{MHz}, \mathrm{CDCl}_{3}\right)$ ): $7.94-7.88(\mathrm{~m}, 1 \mathrm{H}), 7.86-7.80(\mathrm{~m}, 1 \mathrm{H}), 7.47(\mathrm{~s}, 1 \mathrm{H}), 7.40-7.27(\mathrm{~m}$, $10 \mathrm{H}), 7.24-7.18(\mathrm{~m}, 2 \mathrm{H}), 5.89(\mathrm{dd}, J=17.7,10.9 \mathrm{~Hz}, 1 \mathrm{H}), 5.57(\mathrm{~s}, 1 \mathrm{H}), 5.20(\mathrm{dd}, J=11.0,1.1 \mathrm{~Hz}$, $1 \mathrm{H}), 5.20(\mathrm{~s}, 1 \mathrm{H}), 5.14(\mathrm{dd}, J=17.6,1.1 \mathrm{~Hz}, 1 \mathrm{H}), 3.21(\mathrm{~s}, 1 \mathrm{H}), 1.22(\mathrm{~s}, 3 \mathrm{H})$.

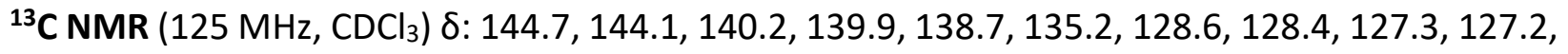
$126.8,126.7,125.1,124.1,123.8,123.7,122.7,118.7,84.0,77.3,75.7,17.9$.

HRMS $(\mathrm{Na}+, m / z)$ for $\mathrm{C}_{26} \mathrm{H}_{24} \mathrm{O}_{2} \mathrm{~S}$ : calcd. $=423.1389 ;$ found $=423.1380$.

FTIR (neat): 3543, 3094, 3025, 2875, 1491, 1452, 1266, 1041, 938, $697 \mathrm{~cm}^{-1}$

HPLC: (Two connected Chiralcel columns OD-H and AD-H, Hexane:2-PrOH $=98: 02,0.5 \mathrm{~mL} / \mathrm{min}$, $210 \mathrm{~nm})$

$[\alpha]_{D}^{24}=+30.0^{\circ}\left(\mathrm{c}=0.2, \mathrm{CHCl}_{3}\right)$

MP: $[111-115]^{\circ} \mathrm{C}$ 

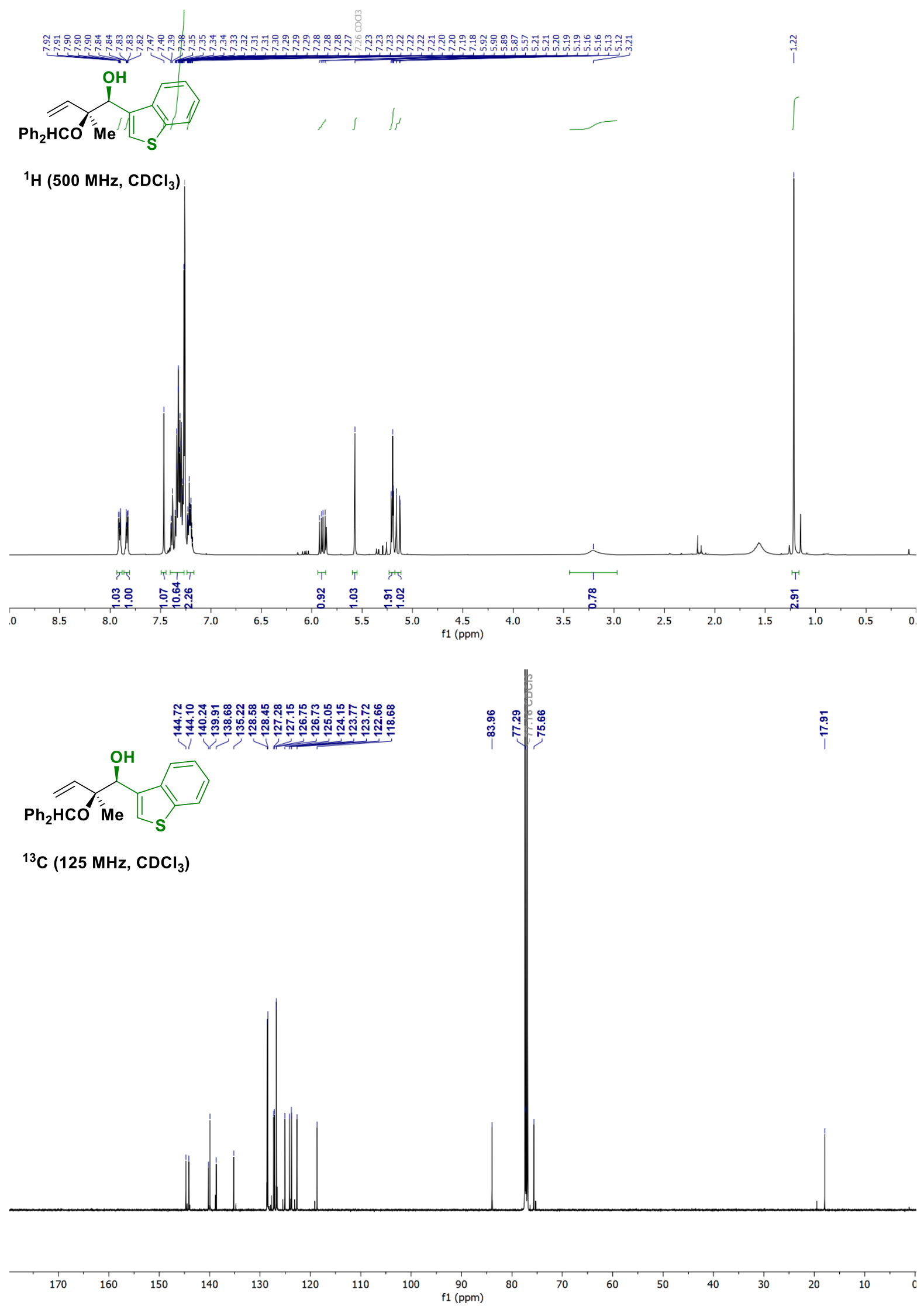

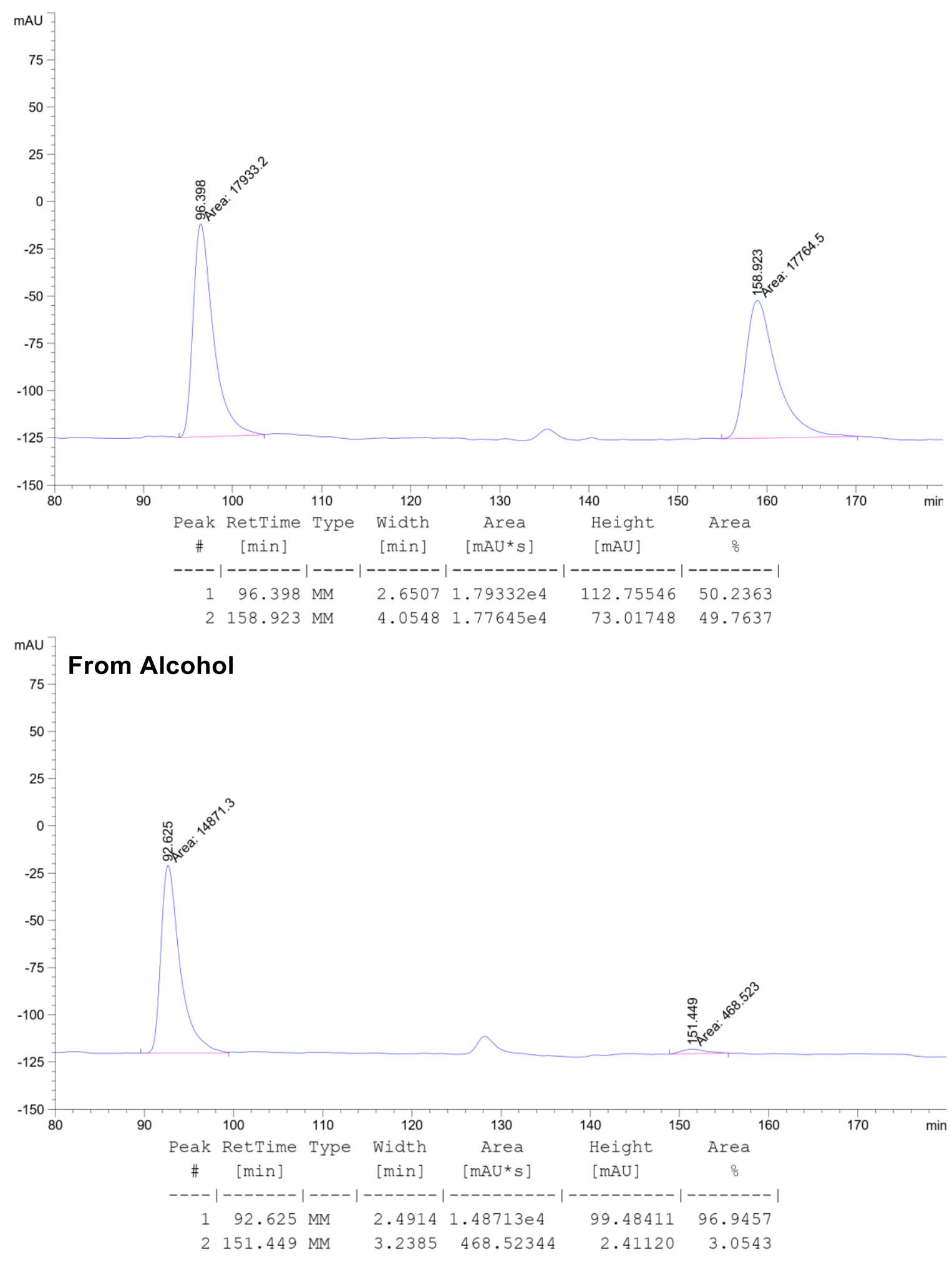


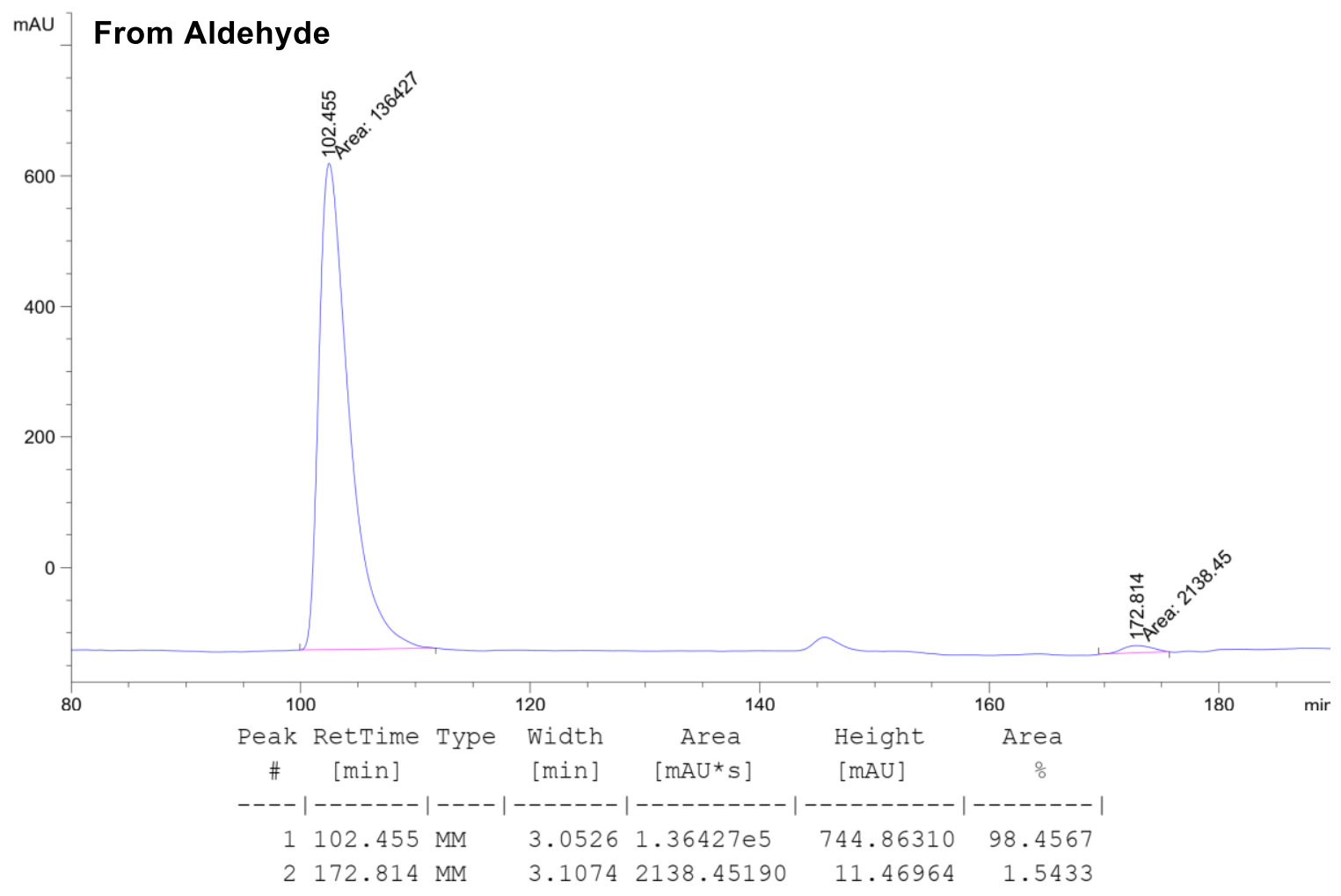


(1S,2S)-2-(benzhydryloxy)-2-methyl-1-(2-(methylthio)benzo[d]thiazol-6-yl)but-3-en-1-ol (4I)

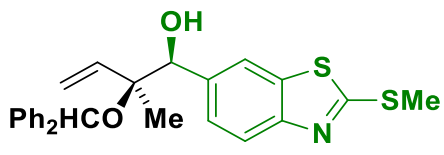

Alcohol oxidation level: Alcohol $21(42.3 \mathrm{mg}, 0.2 \mathrm{mmol})$ was subjected to standard reaction conditions $\left(75^{\circ} \mathrm{C}, 24 \mathrm{~h}\right)$. Upon flash column chromatography $\left(\mathrm{SiO}_{2}, 5: 95 \mathrm{EtOAc}\right.$ :hexanes), the title compound $4 \mathrm{l}$ was obtained as a colorless oil in $72 \%$ yield $(64.5 \mathrm{mg}, 0.144 \mathrm{mmol}, 10: 1 \mathrm{dr}, 89 \%$ ee).

Aldehyde oxidation level: Aldehyde 3l (41.9 mg, $0.2 \mathrm{mmol})$ was subjected to standard reaction conditions $\left(75^{\circ} \mathrm{C}, 24 \mathrm{~h}\right)$. Upon flash column chromatography $\left(\mathrm{SiO}_{2}\right.$, 5:95 EtOAc:hexanes), the title compound $4 \mathrm{l}$ was obtained as a colorless oil in $79 \%$ yield $(70.7 \mathrm{mg}, 0.158 \mathrm{mmol}, 10: 1 \mathrm{dr}, 90 \%$ ee).

$\operatorname{TLC}\left(\mathrm{SiO}_{2}\right) \mathrm{R}_{\mathrm{f}}=0.5$ (15:85 EtOAc:hexanes).

${ }^{1}$ H NMR (500 MHz, $\left.\mathrm{CDCl}_{3}\right) \delta: 7.84-7.70(\mathrm{~m}, 2 \mathrm{H}), 7.42-7.26(\mathrm{~m}, 10 \mathrm{H}), 7.24-7.17(\mathrm{~m}, 2 \mathrm{H}), 5.86$ $(d d, J=17.6,10.9 \mathrm{~Hz}, 1 \mathrm{H}), 5.57(\mathrm{~s}, 1 \mathrm{H}), 5.27(\mathrm{dd}, \mathrm{J}=10.9,0.9 \mathrm{~Hz}, 1 \mathrm{H}), 5.14-5.03(\mathrm{~m}, 1 \mathrm{H}), 4.85(\mathrm{~d}$, $\mathrm{J}=2.5 \mathrm{~Hz}, 1 \mathrm{H}), 3.41(\mathrm{~d}, \mathrm{~J}=2.7 \mathrm{~Hz}, 1 \mathrm{H}), 2.79(\mathrm{~s}, 3 \mathrm{H}), 1.04(\mathrm{~s}, 3 \mathrm{H})$.

${ }^{13} \mathrm{C}$ NMR $\left(125 \mathrm{MHz}, \mathrm{CDCl}_{3}\right) \delta: 168.3,153.1,144.6,144.0,139.8,135.5,134.8,128.6,128.5,127.3$, $127.2,126.8,126.7,126.5,120.2,120.2,118.9,83.9,79.9,77.2,16.9,16.1$.

HRMS $(\mathrm{Na}+, m / z)$ for $\mathrm{C}_{26} \mathrm{H}_{25} \mathrm{NO}_{2} \mathrm{~S}_{2}$ : calcd. = 315.1428; found = 315.1433.

FTIR (neat): 3558, 3004, 1635, 1482, 1276, 1196, 1051, 976, $781 \mathrm{~cm}^{-1}$.

HPLC: (Two connected Chiralcel AD-H columns, Hexane:2-PrOH = 97:03, $0.5 \mathrm{~mL} / \mathrm{min}, 210 \mathrm{~nm}$ )

$[\alpha]_{D}^{24}=+48.0^{\circ}\left(\mathrm{c}=0.1, \mathrm{CHCl}_{3}\right)$. 


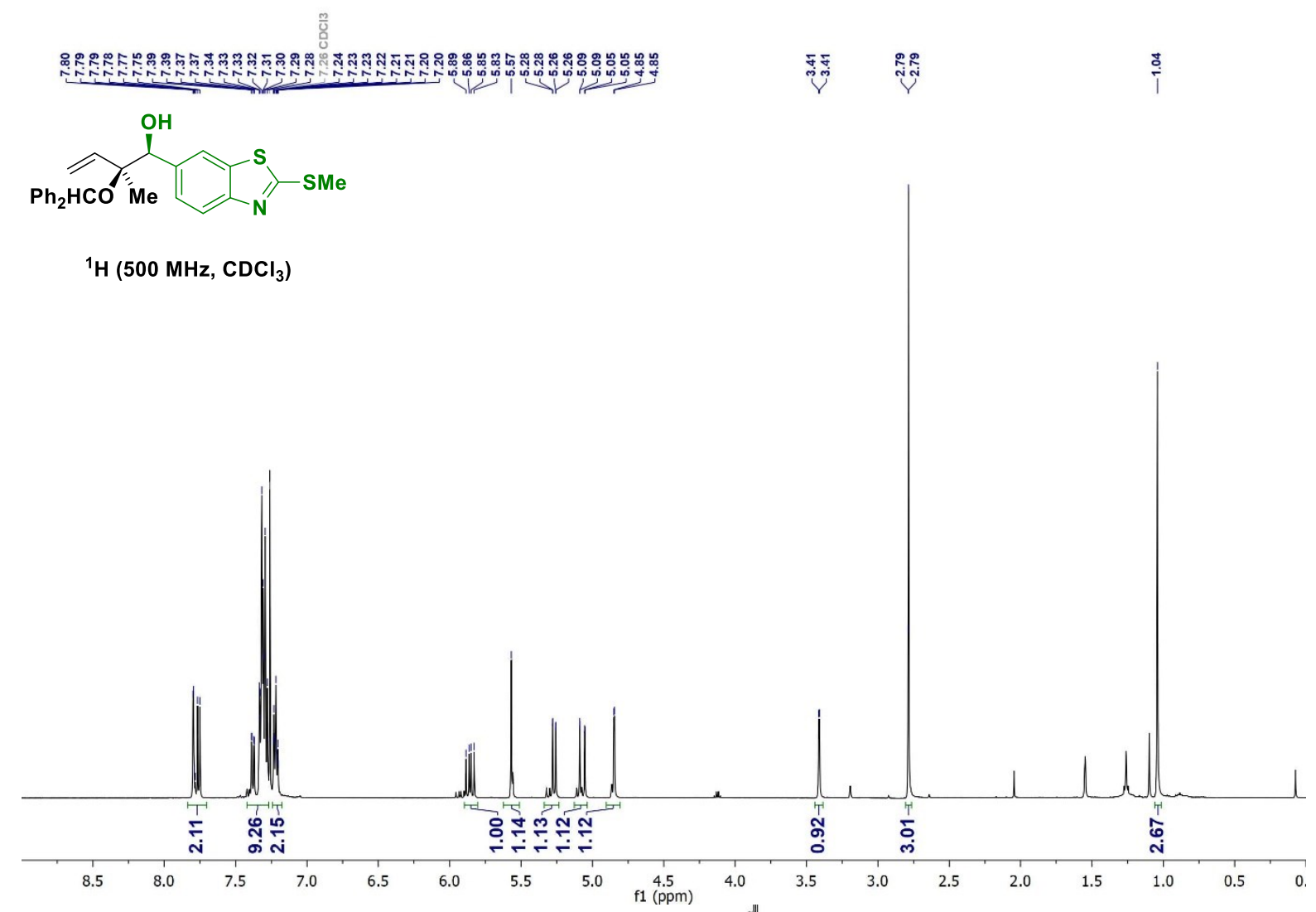

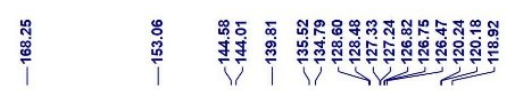

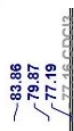

(l)

${ }^{13} \mathrm{C}\left(130 \mathrm{MHz}, \mathrm{CDCl}_{3}\right)$
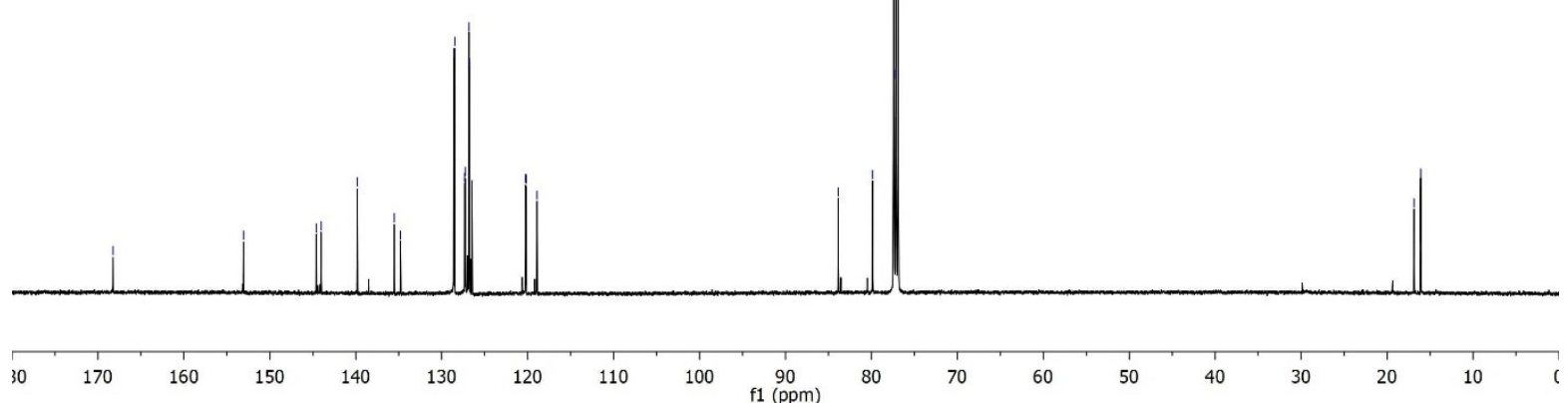

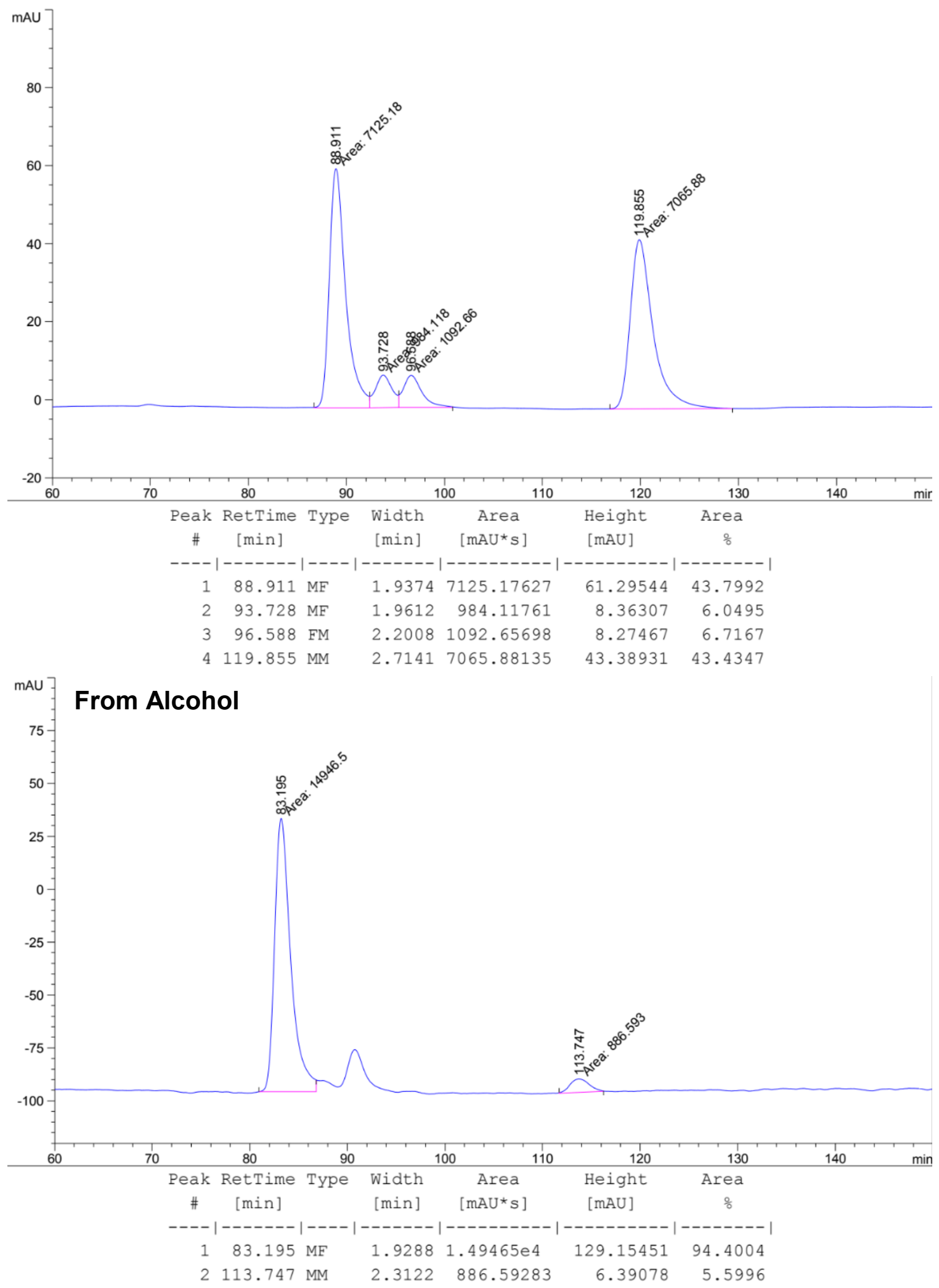


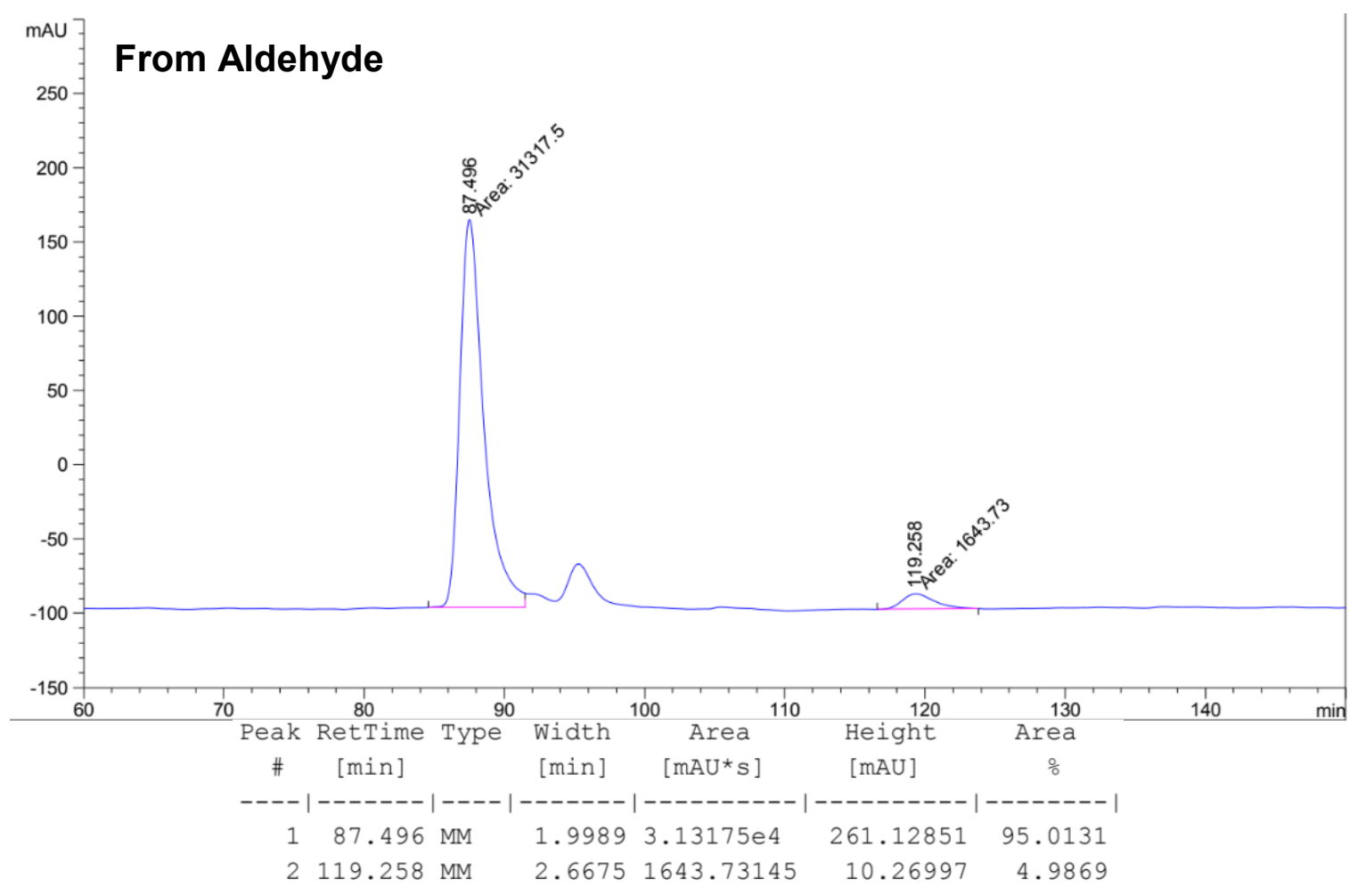


tert-butyl 2-((1S,2S)-2-(benzhydryloxy)-1-hydroxy-2-methylbut-3-en-1-yl)-1H-pyrrole-1carboxylate $(4 \mathrm{~m})$

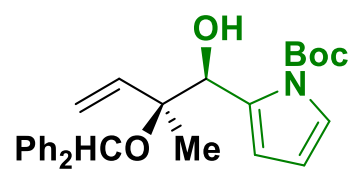

Alcohol oxidation level: Alcohol $2 \mathrm{~m}(39.4 \mathrm{mg}, 0.2 \mathrm{mmol})$ was subjected to standard reaction conditions (10 mol\% catalyst loading, $75{ }^{\circ} \mathrm{C}, \mathbf{4 8} \mathrm{h}$ ). Upon flash column chromatography $\left(\mathrm{SiO}_{2}\right.$, 8:92 EtOAc:hexanes), the title compound $\mathbf{4 m}$ was obtained as a yellow wax in $51 \%$ yield (44.4 $\mathrm{mg}, 0.102 \mathrm{mmol}, 15: 1 \mathrm{dr}, 97 \%$ ee).

Aldehyde oxidation level: Aldehyde $3 \mathrm{~m}(39.0 \mathrm{mg}, 0.2 \mathrm{mmol})$ was subjected to standard reaction conditions $\left(75^{\circ} \mathrm{C}, 24 \mathrm{~h}\right)$. Upon flash column chromatography ( $\mathrm{SiO}_{2}, 8: 92 \mathrm{EtOAc}$ :hexanes), the title compound $4 \mathrm{~m}$ was obtained as a yellow wax in $84 \%$ yield $(73.2 \mathrm{mg}, 0.169 \mathrm{mmol}, 19: 1 \mathrm{dr}, 97 \%$ ee).

$\operatorname{TLC}\left(\mathrm{SiO}_{2}\right) \mathrm{R}_{\mathrm{f}}=0.25(10: 90$ EtOAc:hexanes)

${ }^{1} \mathrm{H}$ NMR $\left(400 \mathrm{MHz}, \mathrm{CDCl}_{3}\right) \delta: 7.43-7.21(\mathrm{~m}, 7 \mathrm{H}), 7.23-7.16(\mathrm{~m}, 3 \mathrm{H}), 6.39(\mathrm{dd}, J=3.5,1.8 \mathrm{~Hz}, 1 \mathrm{H})$, $6.14(\mathrm{t}, J=3.4 \mathrm{~Hz}, 1 \mathrm{H}), 6.06(\mathrm{dd}, J=17.7,11.0 \mathrm{~Hz}, 1 \mathrm{H}), 5.52(\mathrm{~s}, 1 \mathrm{H}), 5.46(\mathrm{~d}, J=6.8 \mathrm{~Hz}, 1 \mathrm{H}), 5.22-$ $5.12(\mathrm{~m}, 2 \mathrm{H}), 3.74(\mathrm{~d}, J=7.0 \mathrm{~Hz}, 1 \mathrm{H}), 1.51(\mathrm{~s}, 9 \mathrm{H}), 1.15(\mathrm{~s}, 3 \mathrm{H})$.

${ }^{13} \mathrm{C}$ NMR $\left(100 \mathrm{MHz}, \mathrm{CDCl}_{3}\right)$ $\delta: 150.0,144.9,144.4,140.5,134.4,128.3,128.3,127.0,127.0,126.9$, $126.9,122.3,117.3,114.3,110.1,84.0,83.4,77.0,73.2,28.1,19.0$.

HRMS $(\mathrm{Na}+, m / z)$ for $\mathrm{C}_{27} \mathrm{H}_{31} \mathrm{NO}_{4}$ : calcd. $=456.2145 ;$ found $=456.2137$.

FTIR (neat): 3426, 2980, 1739, 1322, 1126, 1043, $698 \mathrm{~cm}^{-1}$

HPLC: (Two connected Chiralcel columns AD-H and OD-H, Hexane:2-PrOH = 98:02, $0.5 \mathrm{~mL} / \mathrm{min}$, $210 \mathrm{~nm})$

$[\alpha]_{D}^{24}=-24.0^{\circ}\left(\mathrm{c}=1.0, \mathrm{CHCl}_{3}\right)$ 


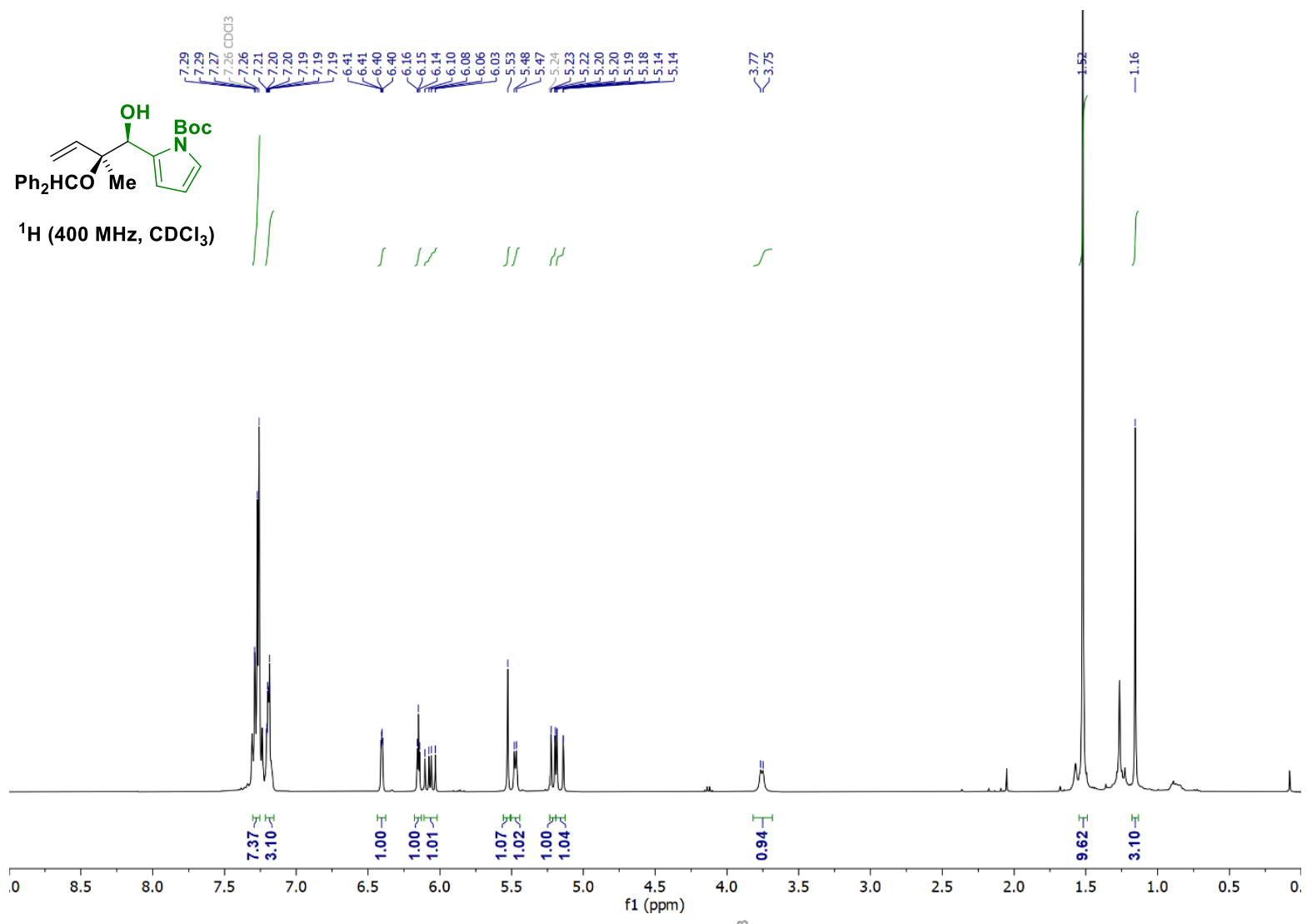

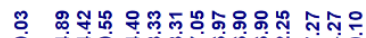

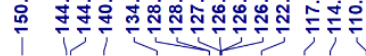

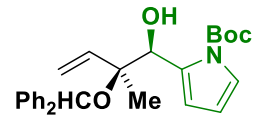

$\frac{20}{5}$

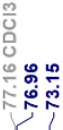

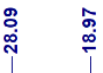

${ }^{13} \mathrm{C}\left(100 \mathrm{MHz}, \mathrm{CDCl}_{3}\right)$

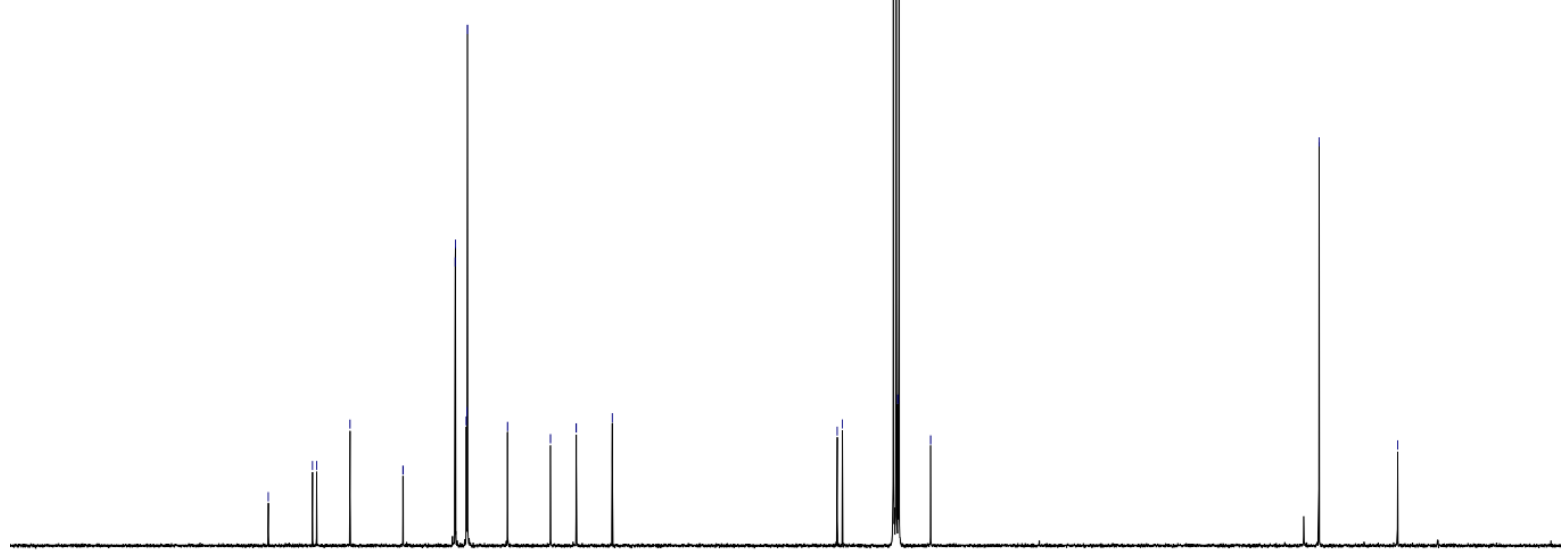

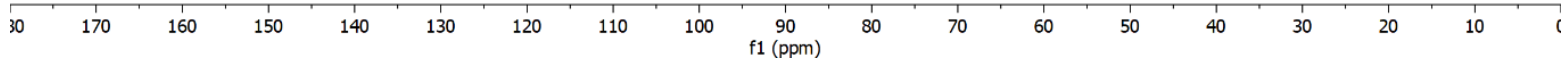



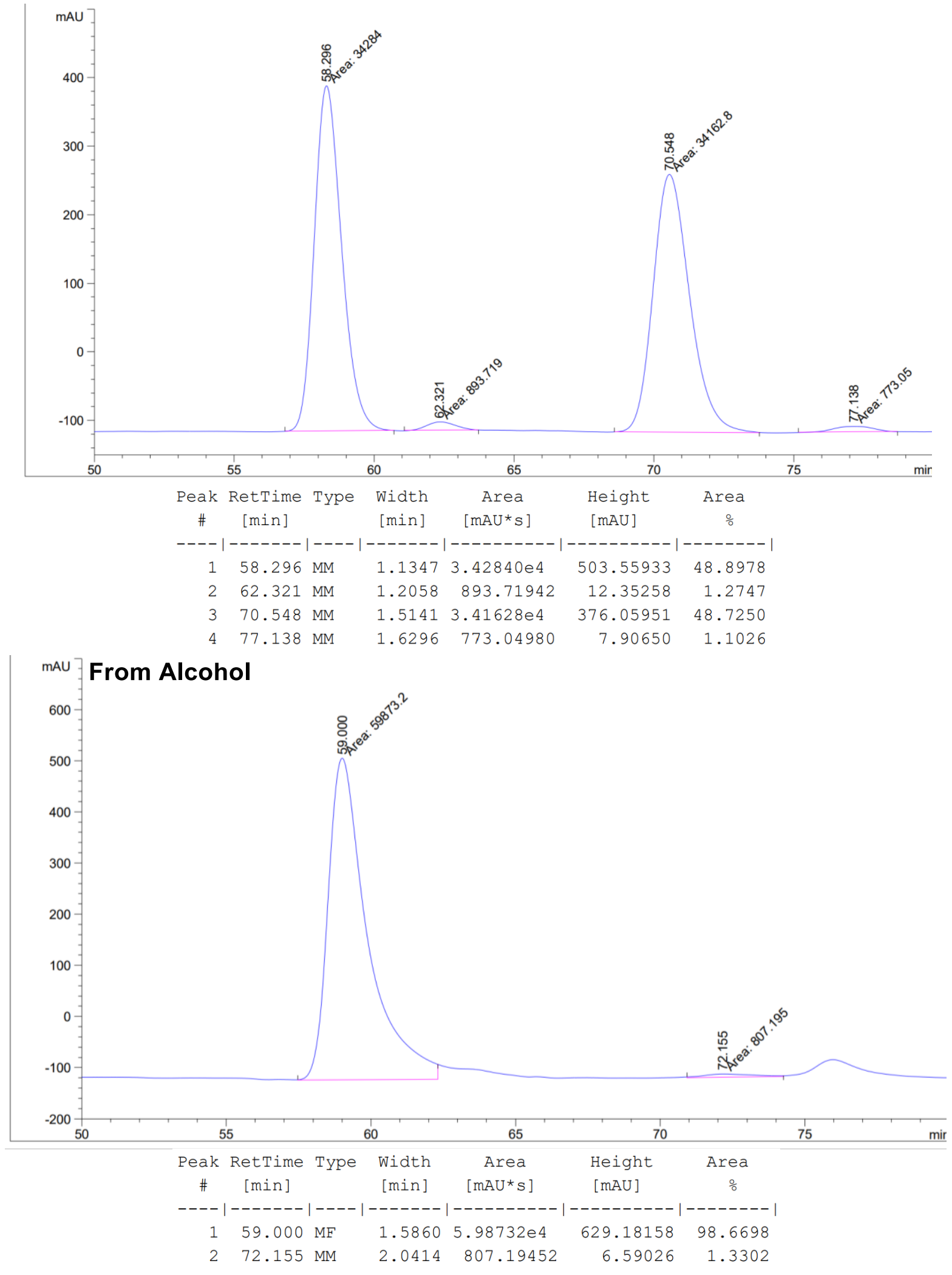


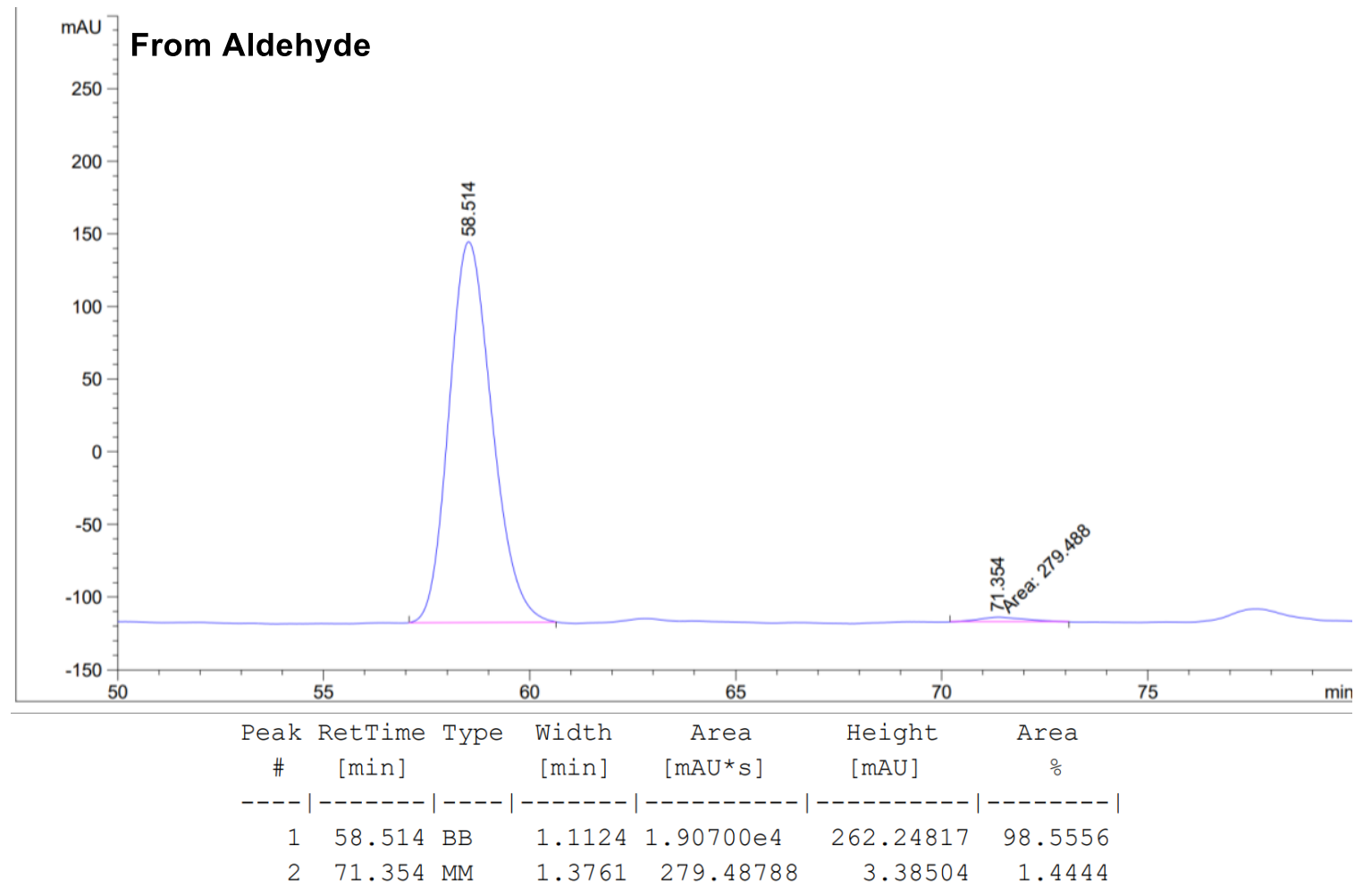




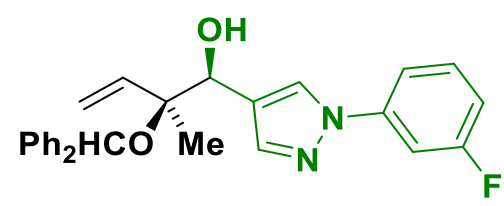

Alcohol oxidation level: Alcohol $2 \mathrm{n}(38.4 \mathrm{mg}, 0.2 \mathrm{mmol})$ was subjected to standard reaction conditions $\left(75^{\circ} \mathrm{C}, 48 \mathrm{~h}\right)$. Upon flash column chromatography $\left(\mathrm{SiO}_{2}, 15: 85\right.$ EtOAc:hexanes), the title compound 4 a was obtained as a yellow oily solid in $70 \%$ yield $(60.0 \mathrm{mg}, 0.14 \mathrm{mmol}, 11: 1 \mathrm{dr}$, $96 \%$ ee).

Aldehyde oxidation level: Aldehyde $3 \mathrm{n}(38.0 \mathrm{mg}, 0.2 \mathrm{mmol})$ was subjected to standard reaction conditions $\left(75^{\circ} \mathrm{C}, 24 \mathrm{~h}\right)$. Upon flash column chromatography $\left(\mathrm{SiO}_{2}, 15: 85\right.$ EtOAc:hexanes), the title compound 4 a was obtained as a yellow oily solid in $85 \%$ yield $(72.8 \mathrm{mg}, 0.17 \mathrm{mmol}, 14: 1 \mathrm{dr}$, $95 \%$ ee).

$\operatorname{TLC}\left(\mathrm{SiO}_{2}\right) \mathrm{R}_{\mathrm{f}}=0.25$ (20:80 EtOAc:hexanes)

${ }^{1} \mathbf{H}$ NMR $\left(500 \mathrm{MHz}, \mathrm{CDCl}_{3}\right) \delta: 7.85(\mathrm{~s}, 1 \mathrm{H}), 7.63(\mathrm{~s}, 1 \mathrm{H}), 7.42-7.28(\mathrm{~m}, 12 \mathrm{H}), 7.26-7.20(\mathrm{~m}, 2 \mathrm{H})$, 6.95 (ddt, $J=9.9,4.2,2.5 \mathrm{~Hz}, 1 \mathrm{H}$ ), 5.88 (dd, $J=17.7,11.0 \mathrm{~Hz}, 1 \mathrm{H}), 5.60(\mathrm{~s}, 1 \mathrm{H}), 5.36$ (dd, $J=10.9$, $1.0 \mathrm{~Hz}, 1 \mathrm{H}), 5.23(\mathrm{dd}, J=17.7,1.0 \mathrm{~Hz}, 1 \mathrm{H}), 4.82(\mathrm{~s}, 1 \mathrm{H}), 3.11(\mathrm{~s}, 1 \mathrm{H}), 1.14(\mathrm{~s}, 3 \mathrm{H})$.

${ }^{13} \mathrm{C}$ NMR (125 MHz, CDCl 3$) \delta: 163.4(\mathrm{~d}, J=246.3 \mathrm{~Hz}), 144.5,143.9,141.6(\mathrm{~d}, J=10.3 \mathrm{~Hz}), 140.6$, 139.7, 130.8 (d, $J=9.2 \mathrm{~Hz}), 128.6,128.5,127.4,127.3,126.9,126.8,125.6,123.2,119.5,114.1$ (d, $J=3.0 \mathrm{~Hz}$ ), 113.1 (d, $J=21.3 \mathrm{~Hz}), 106.6(\mathrm{~d}, J=26.3 \mathrm{~Hz}), 83.5,77.3,73.5,16.7$.

${ }^{19}$ F NMR (470 MHz, $\left.\mathrm{CDCl}_{3}\right) \delta:-111.0$ (ddd, $\left.J=11.0,7.8,4.2 \mathrm{~Hz}\right)$.

HRMS $(\mathrm{Na}+, m / z)$ for $\mathrm{C}_{27} \mathrm{H}_{25} \mathrm{FN}_{2} \mathrm{O}_{2}$ : calcd. $=451.1792 ;$ found $=451.1797$.

FTIR (neat): 2980, 2352, 1275, 1260, 1214, $749 \mathrm{~cm}^{-1}$

HPLC: (Two connected columns Amylose \& AD-H, Hexane:2-PrOH = 95:05, 0.5 mL/min, $210 \mathrm{~nm}$ ) $[\alpha]_{D}^{24}=-7.8^{\circ}\left(\mathrm{c}=0.45, \mathrm{CHCl}_{3}\right)$. 

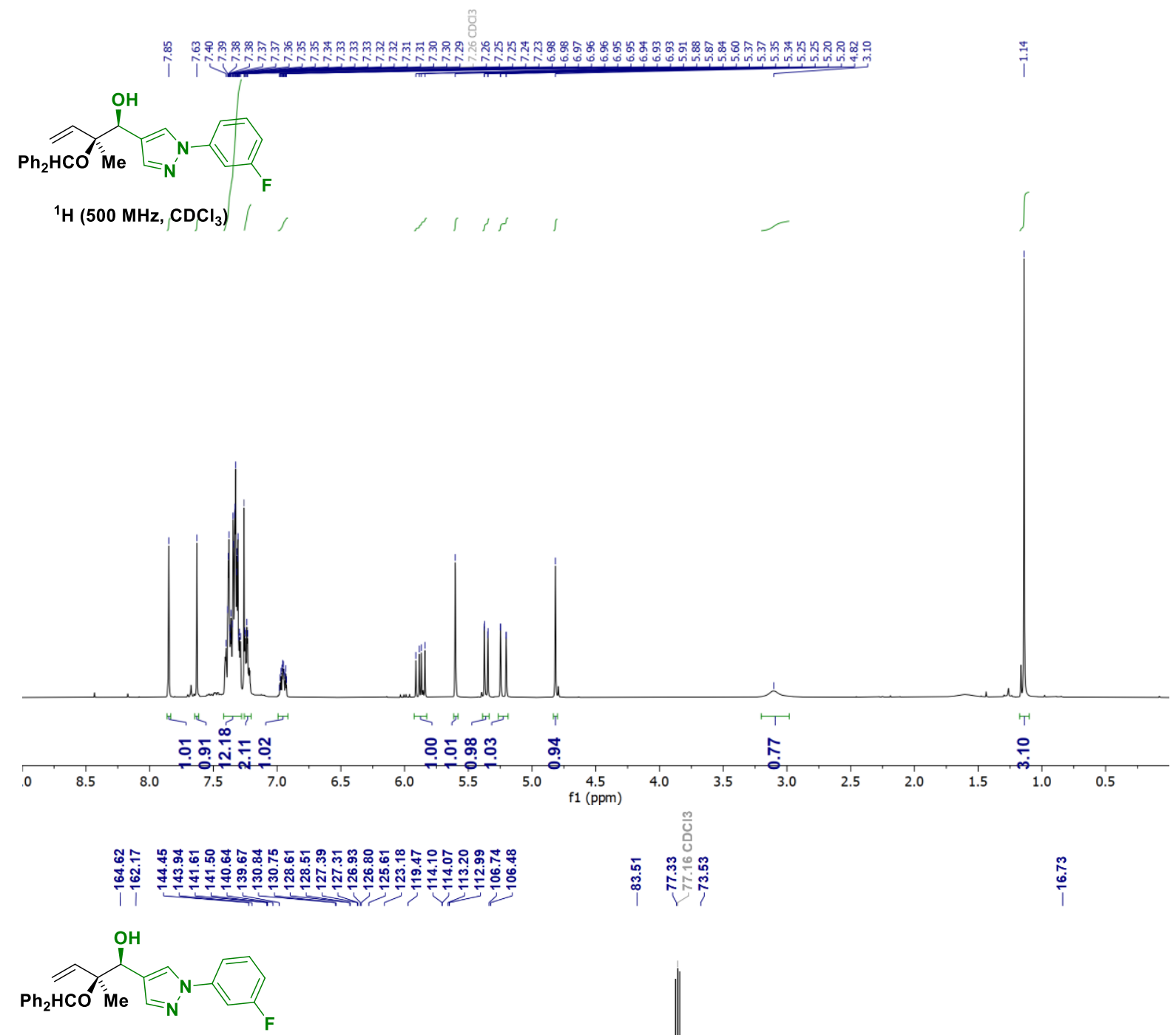

${ }^{13} \mathrm{C}\left(125 \mathrm{MHz}, \mathrm{CDCl}_{3}\right)$

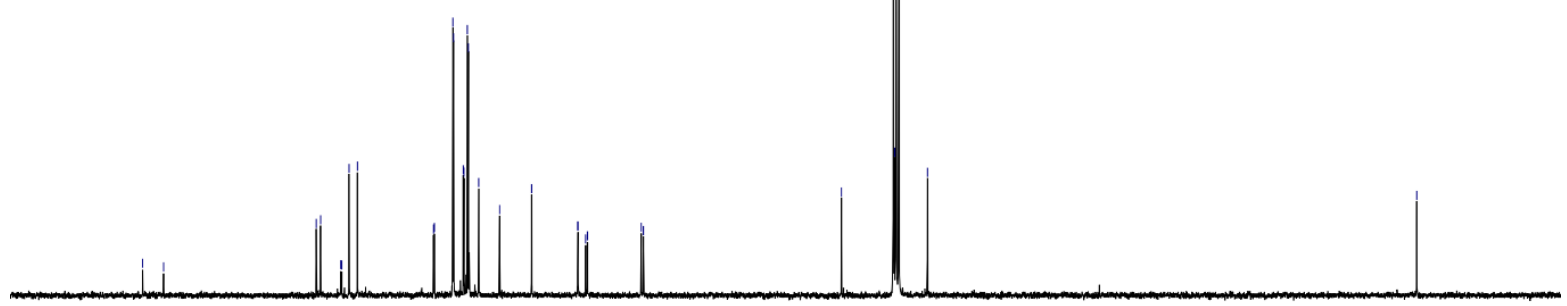

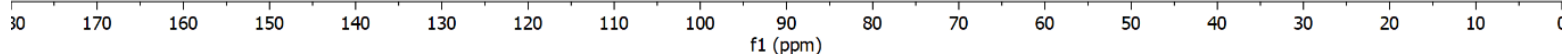




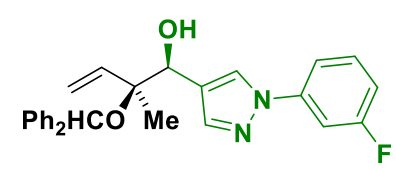

$\left.{ }^{19} \mathrm{~F}\left(470 \mathrm{MHz}^{\mathrm{CDCl}}\right)_{3}\right)$

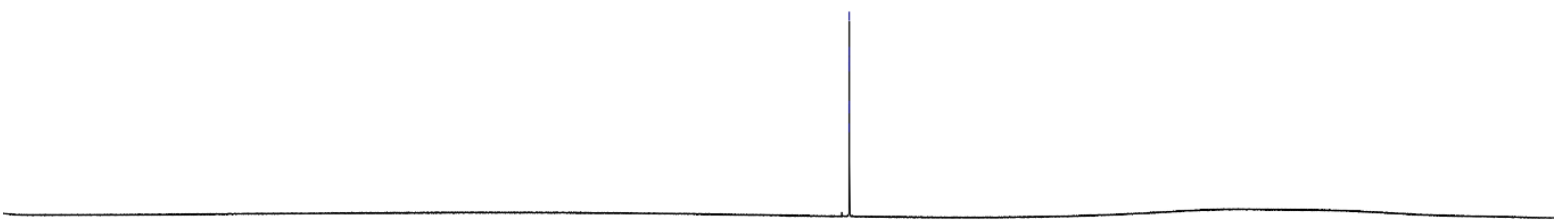

\begin{tabular}{lllllllllllllllllllllllllllllllllll}
10 & 10 & 0 & -10 & -20 & -30 & -40 & -50 & -60 & -70 & -80 & -90 & -100 & -110 & -120 & -130 & -140 & -150 & -160 & -170 & -180 & -190 & -200 & -210 & -22 \\
\hline
\end{tabular} 


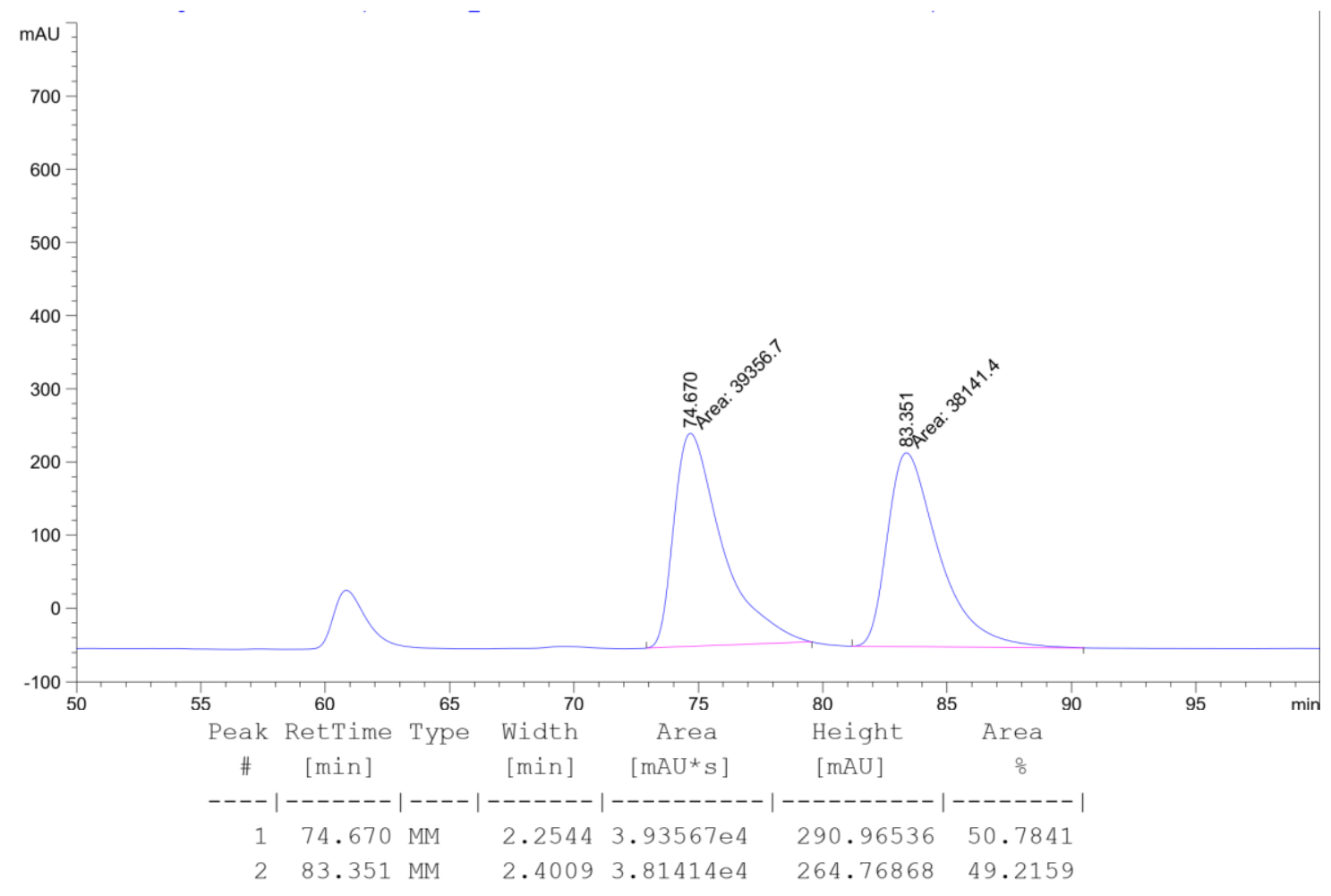

FAU From Alcohol

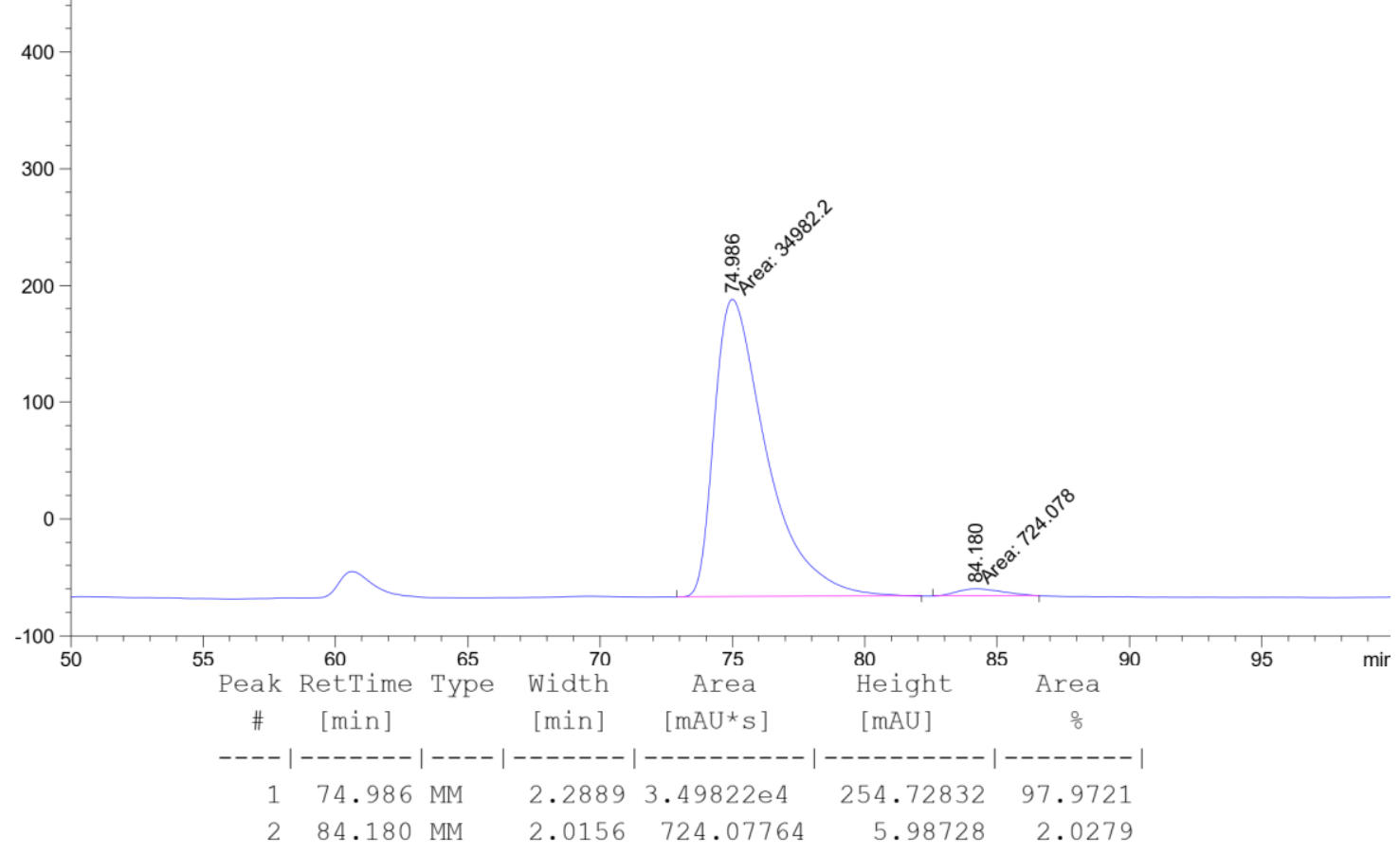




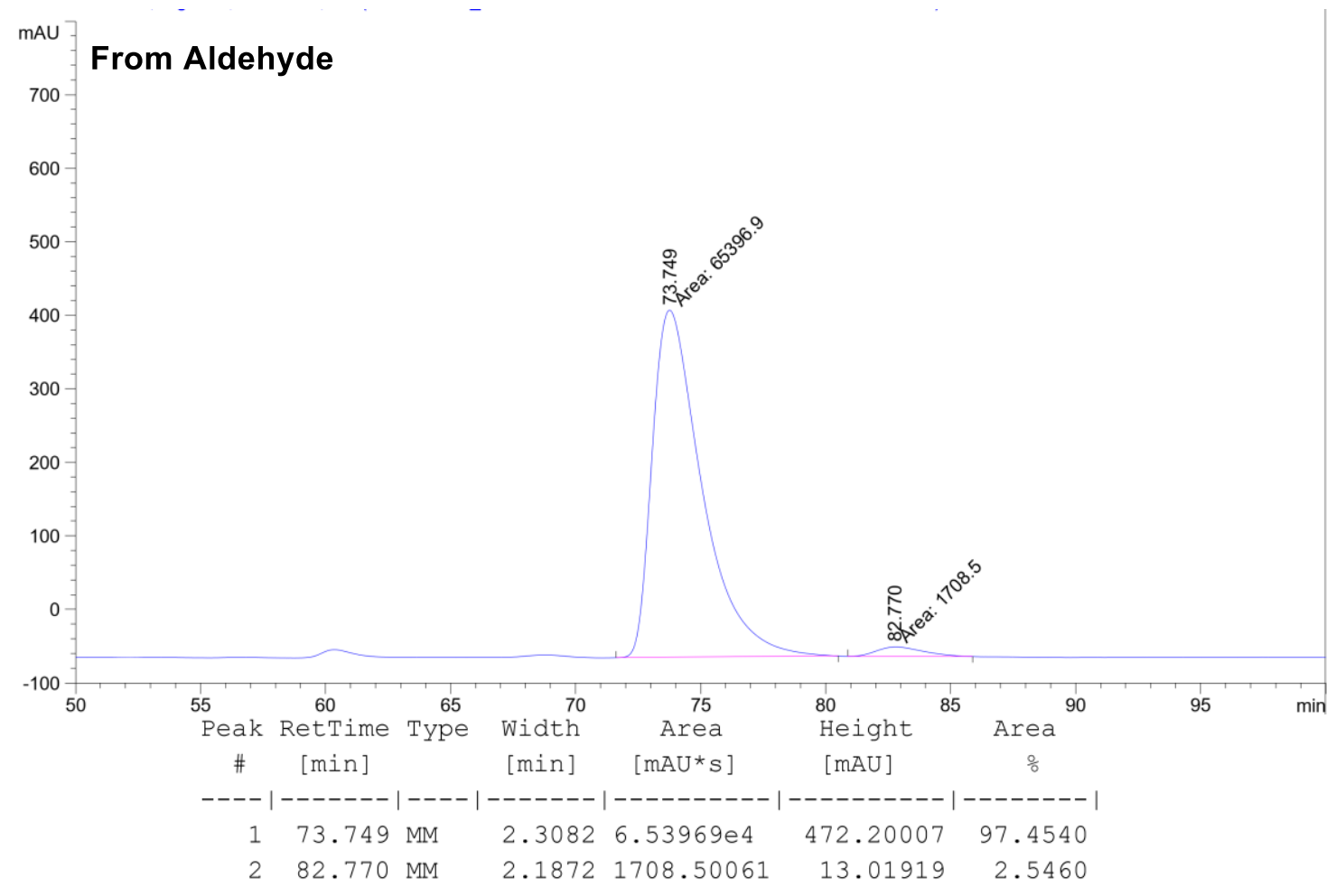


(1S,2S)-2-(benzhydryloxy)-1-(6-methoxypyridin-3-yl)-2-methylbut-3-en-1-ol (40)

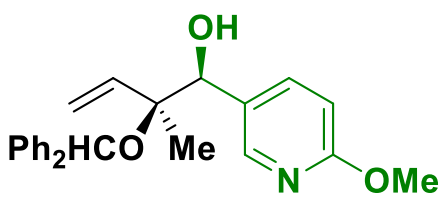

Alcohol oxidation level: Alcohol $20(27.8 \mathrm{mg}, 0.2 \mathrm{mmol})$ was subjected to standard reaction conditions ( 10 mol\% catalyst loading, $75^{\circ} \mathrm{C}, \mathbf{4 8} \mathrm{h}$ ). Upon flash column chromatography $\left(\mathrm{SiO}_{2}\right.$, 13:87 EtOAc:hexanes), the title compound 40 was obtained as an orange solid in $52 \%$ yield (38.9 $\mathrm{mg}, 0.104 \mathrm{mmol}, 9: 1 \mathrm{dr}, 95 \%$ ee).

Aldehyde oxidation level: Aldehyde $30(27.4 \mathrm{mg}, 0.2 \mathrm{mmol})$ was subjected to standard reaction conditions $\left(75^{\circ} \mathrm{C}, 24 \mathrm{~h}\right.$ ). Upon flash column chromatography ( $\mathrm{SiO}_{2}, 13: 87$ EtOAc:hexanes), the title compound 40 was obtained as an orange solid in $87 \%$ yield $(65.6 \mathrm{mg}, 0.174 \mathrm{mmol}, 13: 1 \mathrm{dr}$, $98 \%$ ee).

$\operatorname{TLC}\left(\mathrm{SiO}_{2}\right) \mathrm{R}_{\mathrm{f}}=0.1$ (10:90 EtOAc:hexanes)

${ }^{1} \mathrm{H}$ NMR $\left(500 \mathrm{MHz}, \mathrm{CDCl}_{3}\right) \delta: 8.08(\mathrm{~d}, J=2.3 \mathrm{~Hz}, 1 \mathrm{H}), 7.63(\mathrm{dd}, J=8.6,2.4 \mathrm{~Hz}, 1 \mathrm{H}), 7.34-7.27(\mathrm{~m}$, $8 \mathrm{H}), 7.25-7.19(\mathrm{~m}, 2 \mathrm{H}), 6.68(\mathrm{~d}, J=8.6 \mathrm{~Hz}, 1 \mathrm{H}), 5.82(\mathrm{dd}, J=17.6,10.9 \mathrm{~Hz}, 1 \mathrm{H}), 5.56(\mathrm{~s}, 1 \mathrm{H}), 5.28$ (dd, $J=10.9,1.0 \mathrm{~Hz}, 1 \mathrm{H}$ ), 5.09 (dd, $J=17.7,1.0 \mathrm{~Hz}, 1 \mathrm{H}), 4.71(\mathrm{~d}, J=2.6 \mathrm{~Hz}, 1 \mathrm{H}), 3.92(\mathrm{~s}, 3 \mathrm{H}), 3.28$ (d, $J=2.7 \mathrm{~Hz}, 1 \mathrm{H}), 1.04(\mathrm{~s}, 3 \mathrm{H})$.

${ }^{13} \mathrm{C}$ NMR $\left(125 \mathrm{MHz}, \mathrm{CDCl}_{3}\right)$ $\delta: 163.9,146.4,144.5,144.0,139.6,138.3,128.6,128.5,127.6,127.4$, $127.3,126.8,126.7,119.2,109.9,83.7,77.8,77.2,53.5,16.6$.

HRMS $(\mathrm{Na}+, m / z)$ for $\mathrm{C}_{24} \mathrm{H}_{25} \mathrm{NO}_{3}$ : calcd. = 398.1727; found = 398.1719.

FTIR (neat): 3556, 2981, 2942, 1607, 1491, 1288, 1022, 928, 740, $700 \mathrm{~cm}^{-1}$

HPLC: (Two connected Chiralcel columns AD-H and OD-H, Hexane:2-PrOH = 98:02, 0.5 mL/min, $210 \mathrm{~nm})$

$[\alpha]_{D}^{24}=+3.0^{\circ}\left(\mathrm{c}=0.15, \mathrm{CHCl}_{3}\right)$

MP: [67-71] ${ }^{\circ} \mathrm{C}$ 

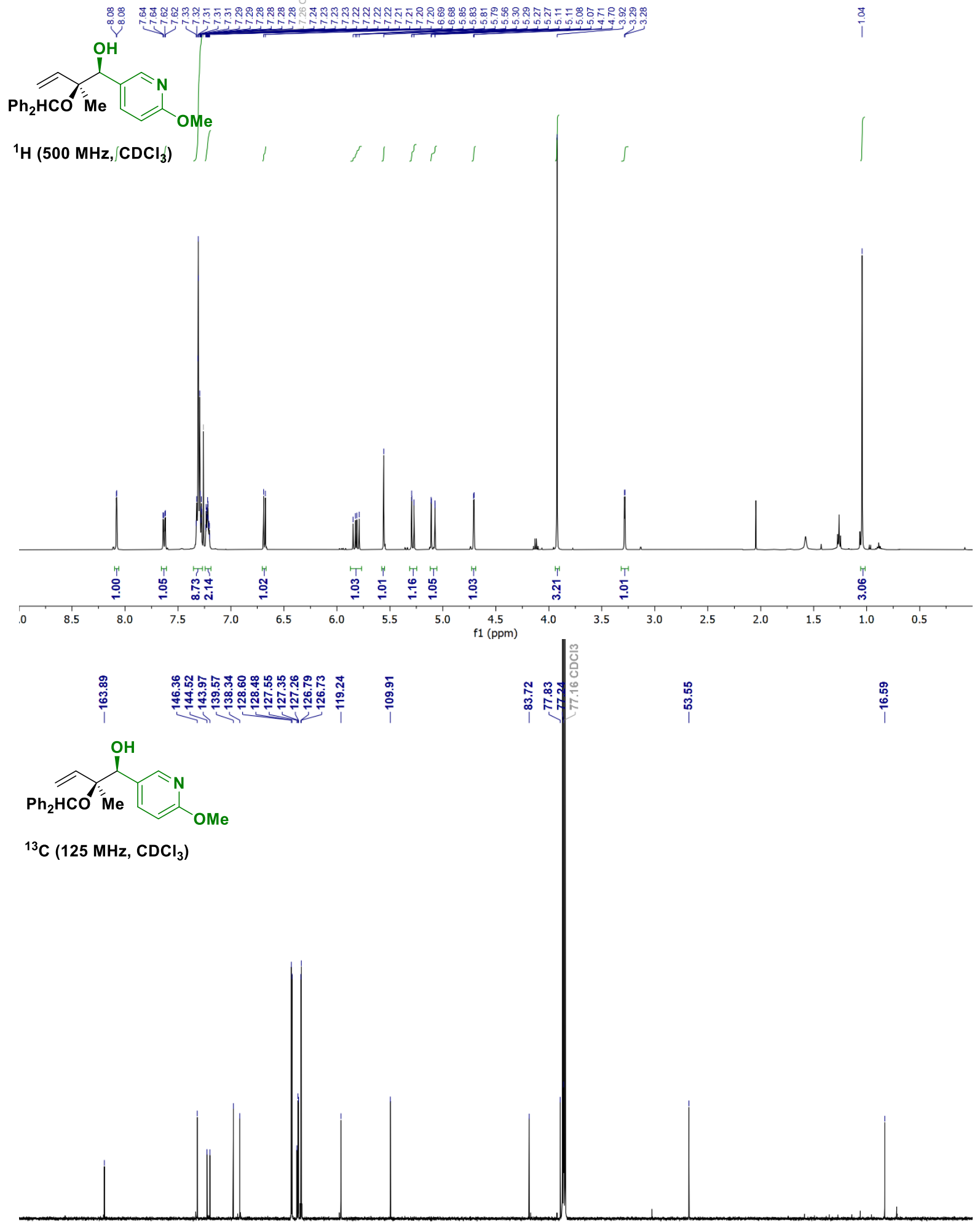

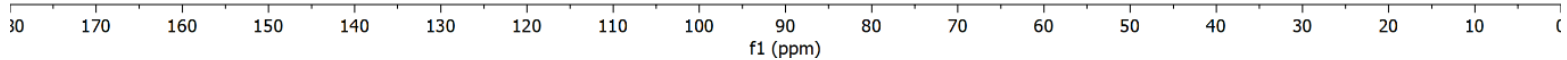



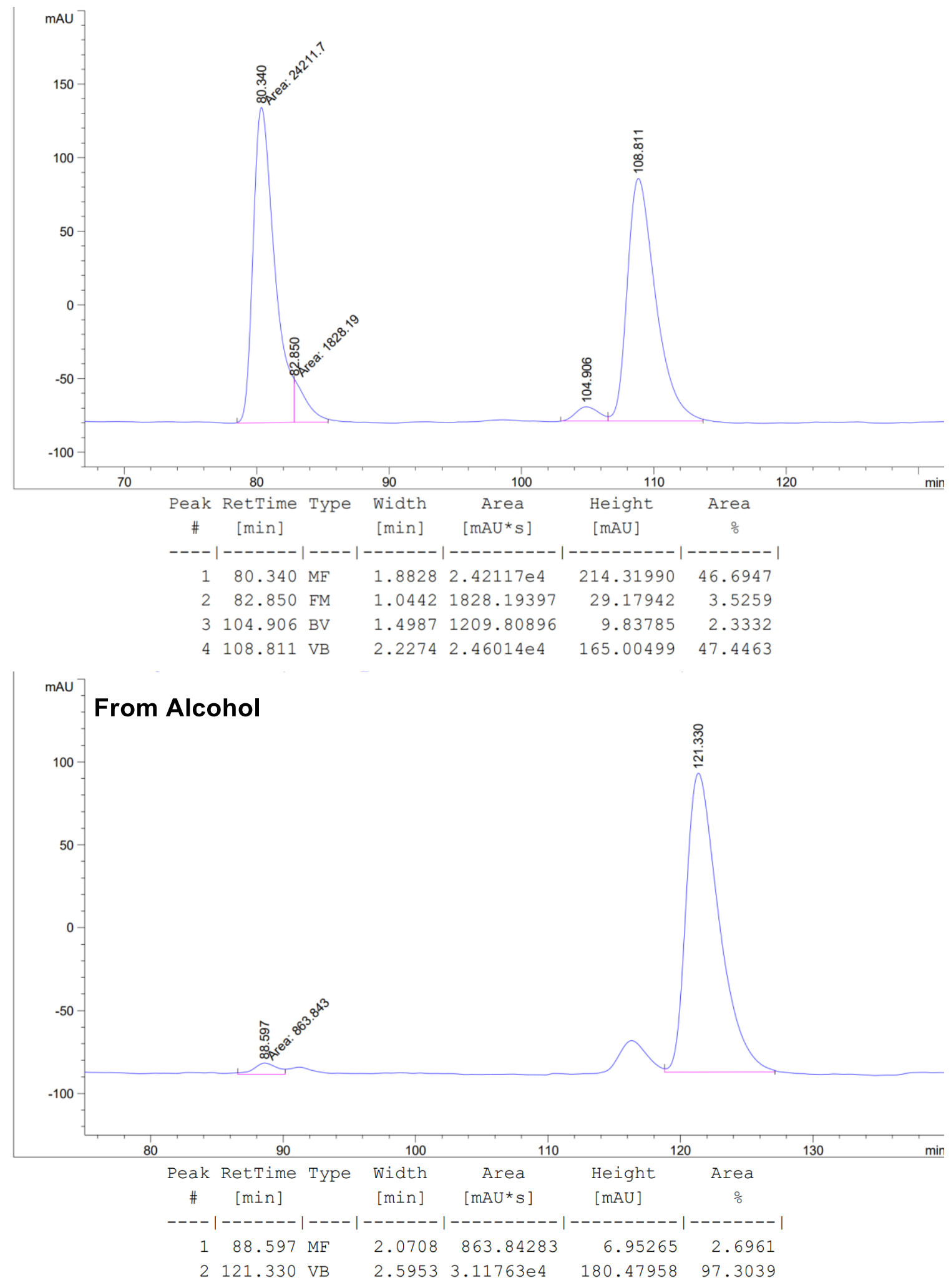


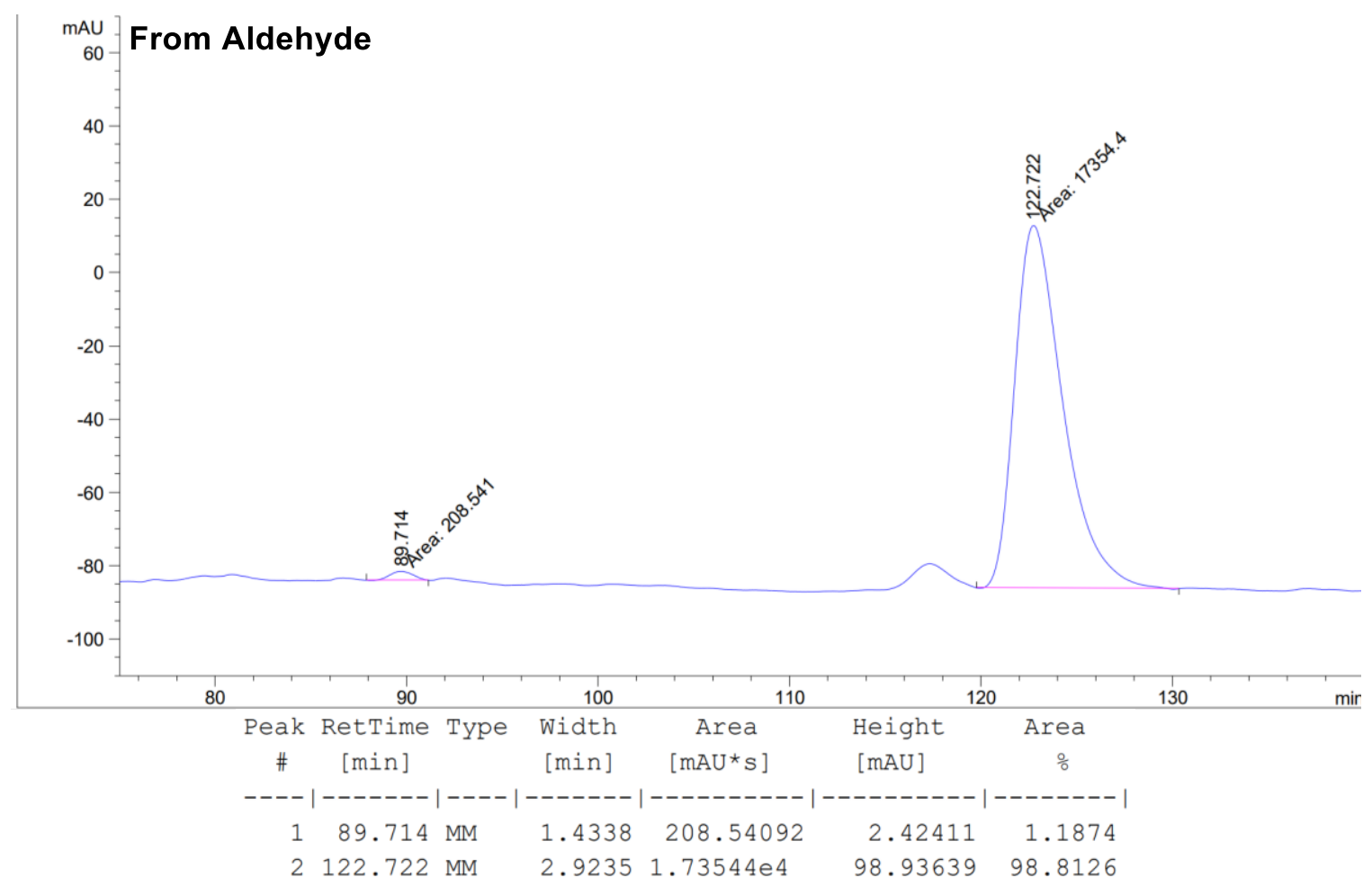


tert-butyl 4-(5-((1S,2S)-2-(benzhydryloxy)-1-hydroxy-2-methylbut-3-en-1-yl)pyrimidin-2yl)piperazine-1-carboxylate (4p)

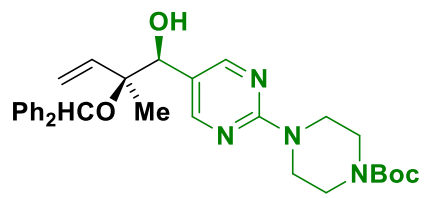

Alcohol oxidation level: Alcohol $\mathbf{2 p}(58.9 \mathrm{mg}, 0.2 \mathrm{mmol})$ was subjected to standard reaction conditions $\left(75^{\circ} \mathrm{C}, 24 \mathrm{~h}\right)$. Upon flash column chromatography $\left(\mathrm{SiO}_{2}, 20: 80\right.$ EtOAc:hexanes), the title compound $4 p$ was obtained as a colorless oil in $91 \%$ yield $(96.6 \mathrm{mg}, 0.182 \mathrm{mmol}, 13: 1 \mathrm{dr}, 96 \%$ ee).

Aldehyde oxidation level: Aldehyde $3 p(58.5 \mathrm{mg}, 0.2 \mathrm{mmol})$ was subjected to standard reaction conditions $\left(75^{\circ} \mathrm{C}, 24 \mathrm{~h}\right)$. Upon flash column chromatography $\left(\mathrm{SiO}_{2}, 20: 80\right.$ EtOAc:hexanes), the title compound $4 \mathrm{p}$ was obtained as a colorless oil in $93 \%$ yield $(98.7 \mathrm{mg}, 0.186 \mathrm{mmol}, 13: 1 \mathrm{dr}$, $96 \%$ ee).

$\operatorname{TLC}\left(\mathrm{SiO}_{2}\right) \mathrm{R}_{\mathrm{f}}=0.5$ (40:60 EtOAc:hexanes).

${ }^{1} \mathrm{H}$ NMR $\left(500 \mathrm{MHz}, \mathrm{CDCl}_{3}\right) \delta: 8.29(\mathrm{~s}, 2 \mathrm{H}), 7.33-7.25(\mathrm{~m}, 9 \mathrm{H}), 7.22$ (dddd, J = 9.4, 6.9, 4.7, $2.8 \mathrm{~Hz}$, $2 \mathrm{H}), 5.79(\mathrm{dd}, \mathrm{J}=17.6,10.9 \mathrm{~Hz}, 1 \mathrm{H}), 5.56(\mathrm{~s}, 1 \mathrm{H}), 5.31(\mathrm{~d}, \mathrm{~J}=10.9 \mathrm{~Hz}, 1 \mathrm{H}), 5.12(\mathrm{~d}, \mathrm{~J}=17.6 \mathrm{~Hz}, 1 \mathrm{H})$, $4.61(\mathrm{~d}, \mathrm{~J}=2.5 \mathrm{~Hz}, 1 \mathrm{H}), 3.78(\mathrm{dd}, \mathrm{J}=6.6,3.9 \mathrm{~Hz}, 4 \mathrm{H}), 3.49(\mathrm{t}, \mathrm{J}=5.3 \mathrm{~Hz}, 5 \mathrm{H}), 3.23(\mathrm{~d}, \mathrm{~J}=2.5 \mathrm{~Hz}$, $1 \mathrm{H}), 1.49(\mathrm{~s}, 9 \mathrm{H}), 1.08(\mathrm{~s}, 3 \mathrm{H})$.

${ }^{13} \mathrm{C}$ NMR $\left(125 \mathrm{MHz}, \mathrm{CDCl}_{3}\right) \delta: 161.5,157.6,157.3,155.0,144.4,143.9,139.3,128.6,128.5,127.4$, $127.3,126.7,126.7,120.6,119.6,83.6,80.1,77.3,76.7,43.9,28.6,16.4$.

HRMS $(\mathrm{Na}+, m / z)$ for $\mathrm{C}_{31} \mathrm{H}_{38} \mathrm{~N}_{4} \mathrm{O}_{4}$ : calcd. = 531.2966; found $=531.2978$.

FTIR (neat): 3459, 2977, 2861, 1692, 1601, 1543, 1243, 1164, 997, $736 \mathrm{~cm}^{-1}$.

HPLC: (Two connected Chiralcel AD-H and ASH columns, Hexane:2-PrOH = 95:05, $0.5 \mathrm{~mL} / \mathrm{min}$, $254 \mathrm{~nm})$

$[\alpha]_{D}^{24}=-68.0^{\circ}\left(\mathrm{c}=0.1, \mathrm{CHCl}_{3}\right)$. 

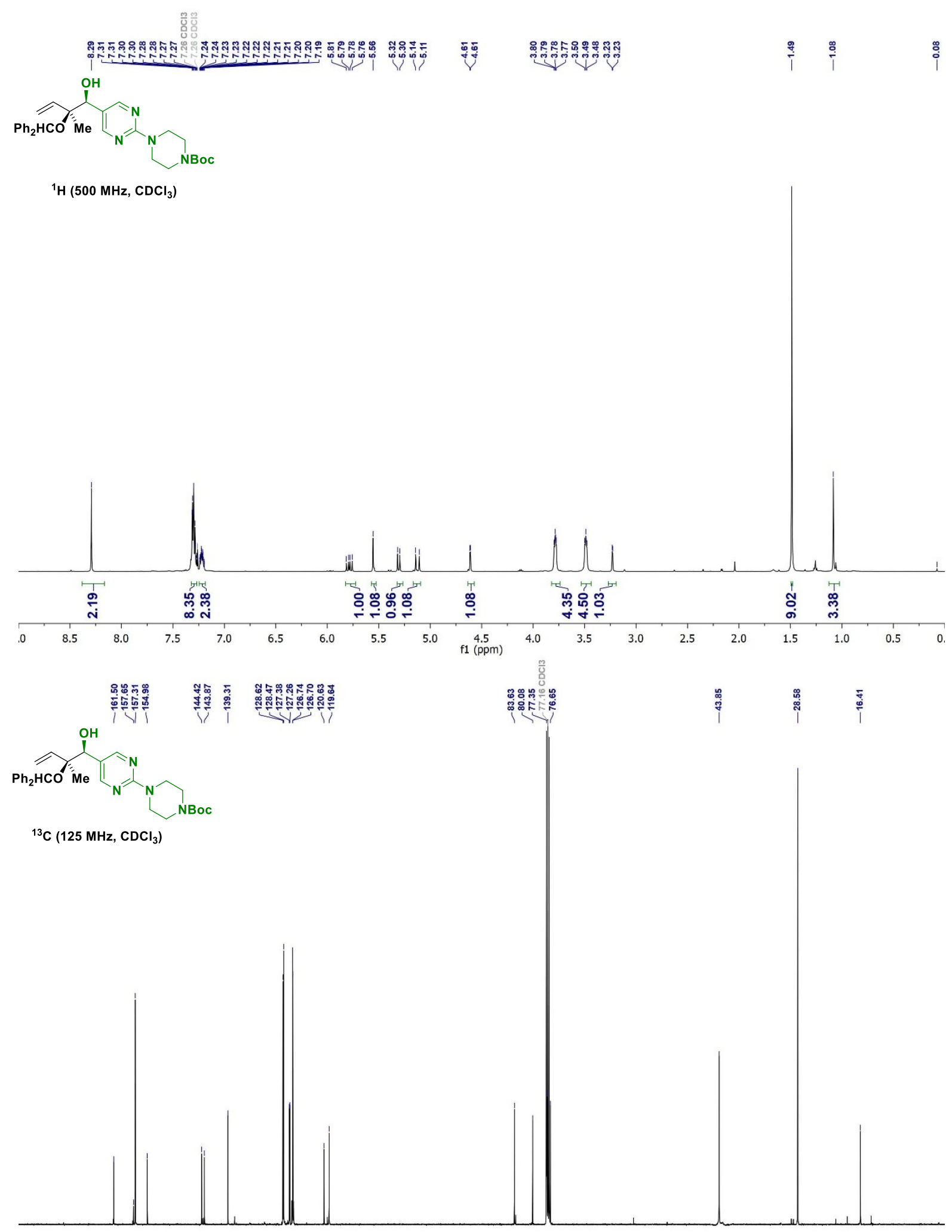

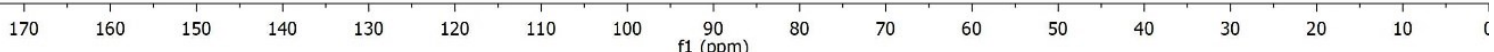



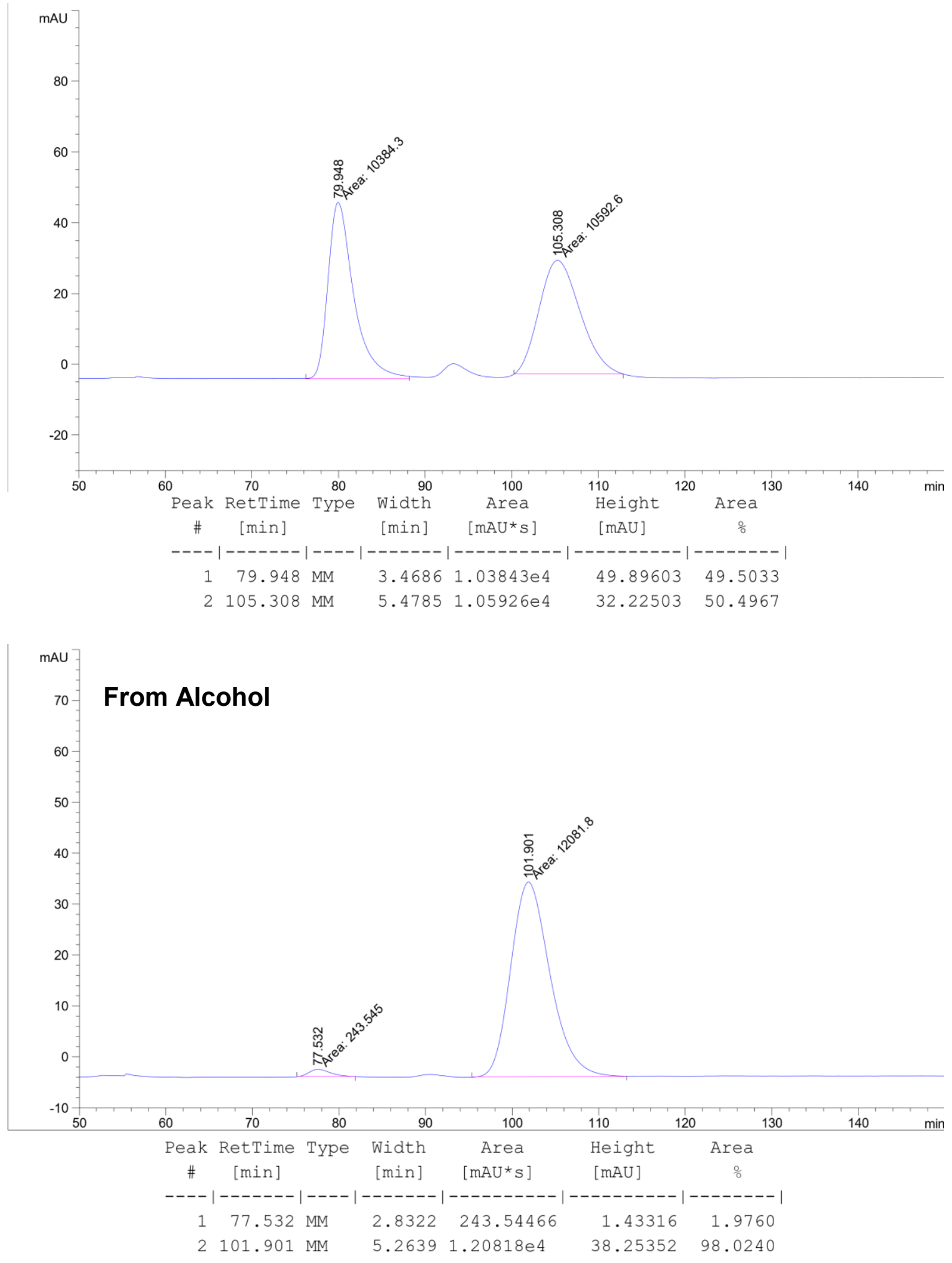


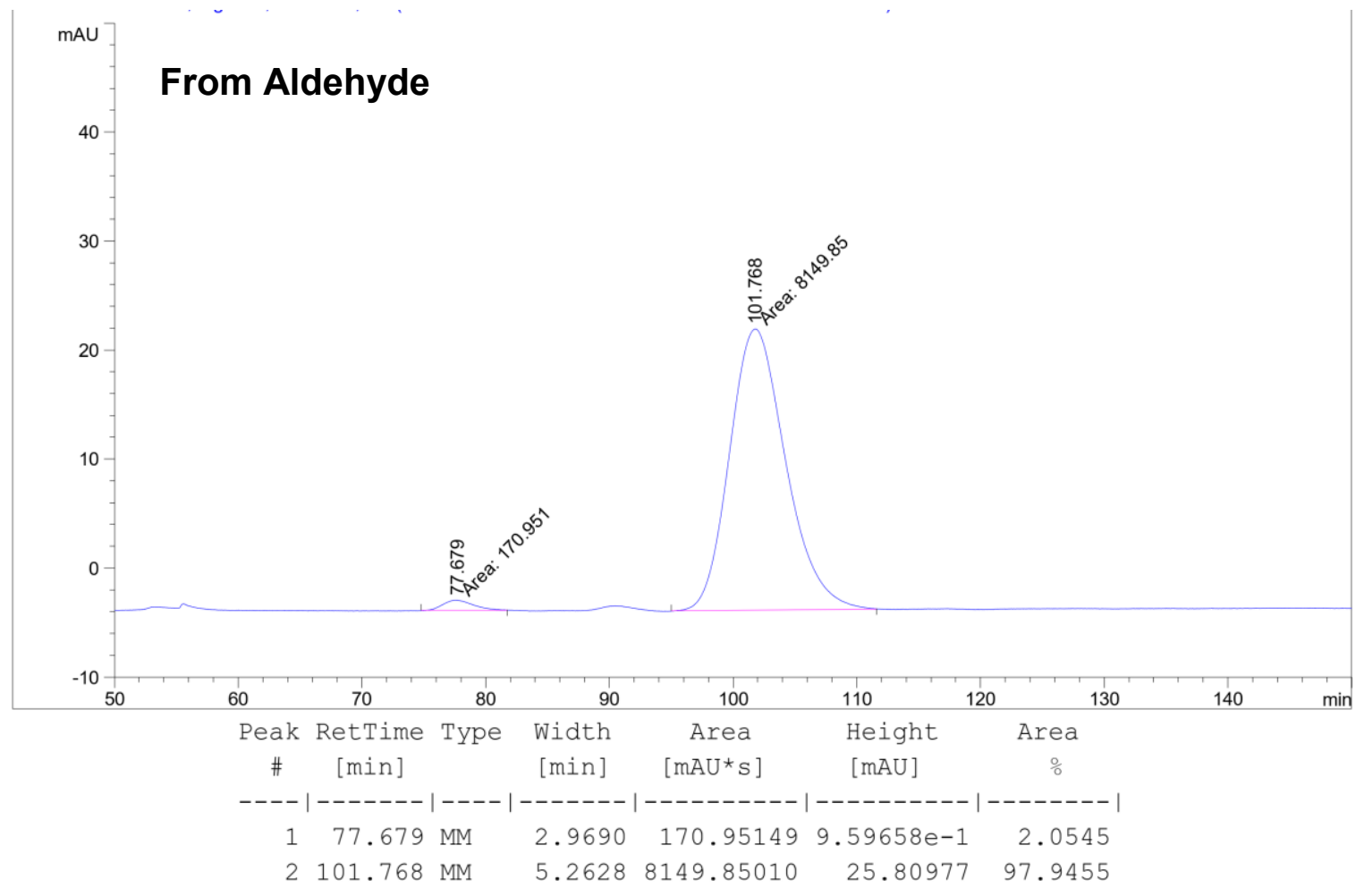




\section{Procedures and Spectral Data for the Coupling Products of Allene and}

Aliphatic Aldehydes 4q-iso-4ji

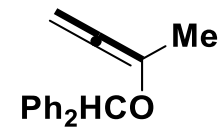

(200 mol\%)
$\mathrm{RuH}_{2}(\mathrm{CO})\left(\mathrm{PPh}_{3}\right)_{3}(7.5 \mathrm{~mol} \%)$

(R)-BINAP (7.5 mol\%)

${ }^{O}$

(100 mol\%)

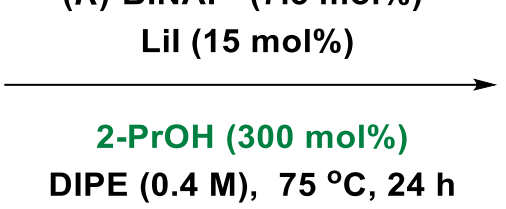

\section{General Procedure for Aliphatic aldehydes}

At aldehyde oxidation level: To a dried pressure tube with a magnetic stir bar under an argon atmosphere charged with Allene $(95.0 \mathrm{mg}, 0.4 \mathrm{mmol}, 200 \mathrm{~mol} \%)$, aldehyde 3q-3jj $(0.2 \mathrm{mmol})$, $\mathbf{R u H}_{\mathbf{2}}(\mathbf{C O})\left(\mathbf{P P h}_{3}\right)_{3}$ (13.8 mg, $\left.0.015 \mathrm{mmol}, 7.5 \mathrm{~mol} \%\right)$, (R)-BINAP (9.4 mg, $0.015 \mathrm{mmol}, 7.5 \mathrm{~mol} \%$ ), Lil (4.0 mg, $0.03 \mathrm{mmol}, 15 \mathrm{mmol} \%$ ), and IPA (45 uL, $0.6 \mathrm{mmol}, 300 \mathrm{~mol} \%$ ) was added DIPE (Diisopropyl ether) $(0.5 \mathrm{~mL}, 0.4 \mathrm{M})$. The tube was sealed with a PTFE lined cap and the reaction mixture was allowed to stir for 24 hours at $75{ }^{\circ} \mathrm{C}$. After reaching ambient temperature, the solvent was removed in vacuo and the residue was subjected to flash column chromatography $\left(\mathrm{SiO}_{2}\right)$ under the noted conditions to furnish the products $\mathbf{4 q - i s o - 4 j j}$. The $\mathrm{dr}$ values were determined by ${ }^{1} \mathrm{H}$ NMR after purification and the enantiomeric excesses were determined by HPLC using chiral columns.

Racemic reactions were performed using (rac)-BINAP as the ligand under identical conditions. 
(1S,2S)-2-(benzhydryloxy)-1-cyclopropyl-2-methylbut-3-en-1-ol (4q)

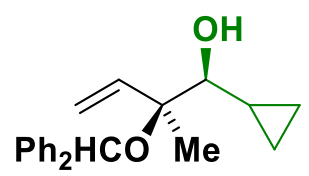

Aldehyde oxidation level: Aldehyde $3 q(14.0 \mathrm{mg}, 0.2 \mathrm{mmol})$ was subjected to standard reaction conditions $\left(75^{\circ} \mathrm{C}, 24 \mathrm{~h}\right)$. Upon flash column chromatography $\left(\mathrm{SiO}_{2}, 5: 95\right.$ EtOAc:hexanes) the title compound $\mathbf{4 q}$ was obtained as a colorless oil in $72 \%$ yield $(44.4 \mathrm{mg}, 0.144 \mathrm{mmol}, 11: 1 \mathrm{dr}, 97 \%$ ee).

$\operatorname{TLC}\left(\mathrm{SiO}_{2}\right) \mathrm{R}_{\mathrm{f}}=0.30$ (10:90 EtOAc:hexanes)

${ }^{1} \mathbf{H}$ NMR $\left(500 \mathrm{MHz}, \mathrm{CDCl}_{3}\right) \delta: 7.32-7.27(\mathrm{~m}, 4 \mathrm{H}), 7.25-7.21(\mathrm{~m}, 4 \mathrm{H}), 7.20-7.13(\mathrm{~m}, 2 \mathrm{H}), 5.81$ (dd, J = 17.6, $10.9 \mathrm{~Hz}, 1 \mathrm{H}), 5.52(\mathrm{~s}, 1 \mathrm{H}), 5.26-5.21(\mathrm{~m}, 2 \mathrm{H}), 3.01(\mathrm{dd}, J=7.8,3.6 \mathrm{~Hz}, 1 \mathrm{H}), 2.66(\mathrm{~d}$, $J=3.6 \mathrm{~Hz}, 1 \mathrm{H}$ ), $1.22(\mathrm{~s}, 3 \mathrm{H}), 0.87$ (qt, $J=8.1,5.0 \mathrm{~Hz}, 1 \mathrm{H}), 0.45$ (dddd, $J=12.7,8.4,6.9,3.7 \mathrm{~Hz}, 2 \mathrm{H}$ ), 0.37 (ddd, $J=9.2,5.0,1.8 \mathrm{~Hz}, 1 \mathrm{H}), 0.29-0.21(\mathrm{~m}, 1 \mathrm{H})$.

${ }^{13} \mathrm{C}$ NMR $\left(125 \mathrm{MHz}, \mathrm{CDCl}_{3}\right) \delta: 145.0,144.4,140.8,128.5,128.4,127.2,127.1,126.8,126.7,117.6$, 83.6, 81.1, 76.6, 17.1, 12.1, 3.7, 2.0.

HRMS $(\mathrm{Na}+, m / z)$ for $\mathrm{C}_{21} \mathrm{H}_{24} \mathrm{O}_{2}$ : calcd. = 331.1669; found $=331.1676$.

FTIR (neat): 2989, 2358, 1453, 1275, 1268, 1085, 1042, 1024, $922 \mathrm{~cm}^{-1}$

HPLC: (Two connected Chiralcel columns AD-H and OD-H, Hexane:2-PrOH = 99:01, 0.5 mL/min, $210 \mathrm{~nm})$

$[\alpha]_{D}^{24}=+13.5^{\circ}\left(\mathrm{c}=0.26, \mathrm{CHCl}_{3}\right)$. 


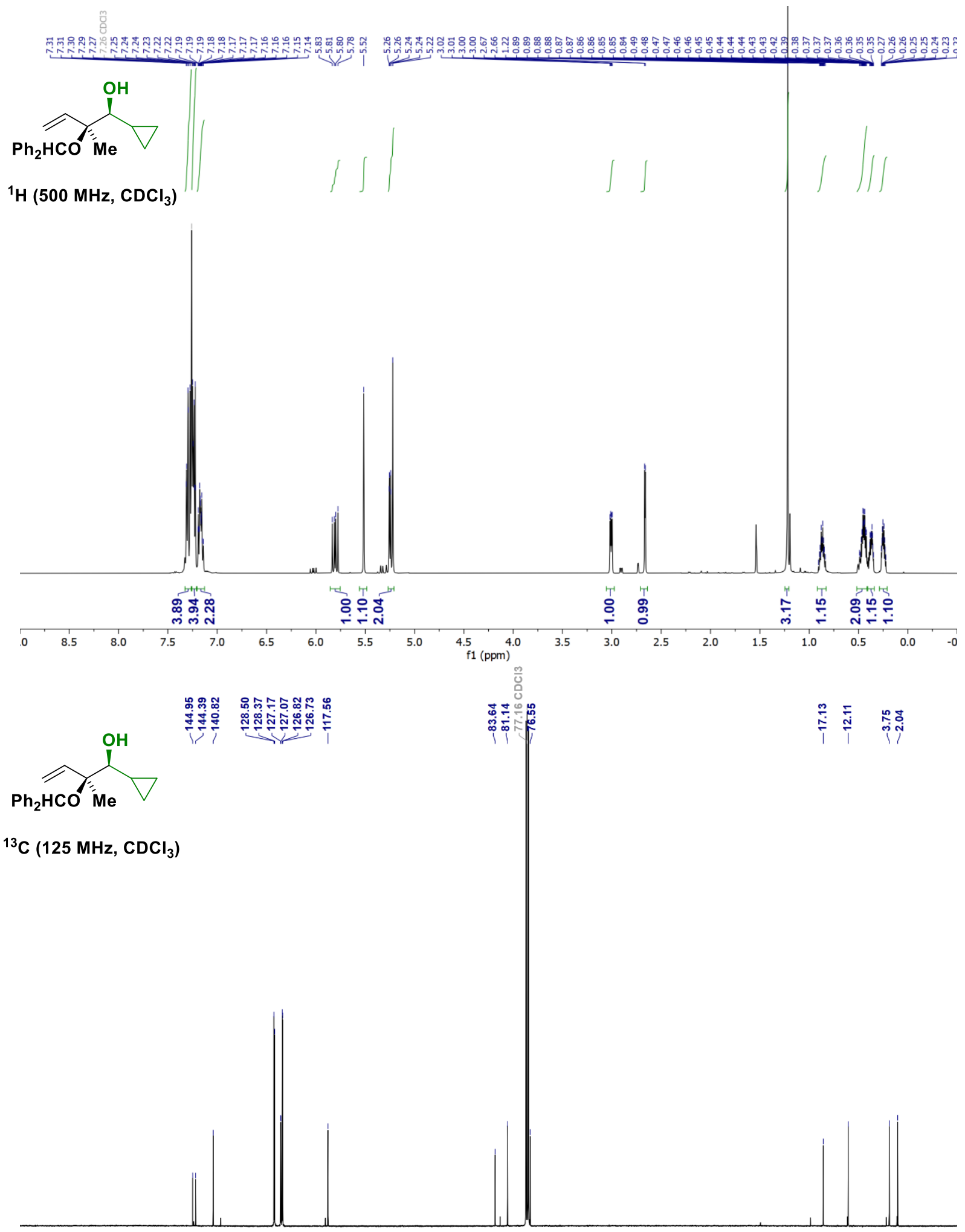

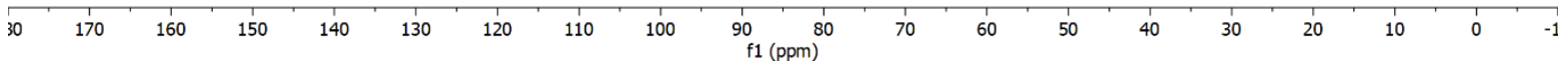



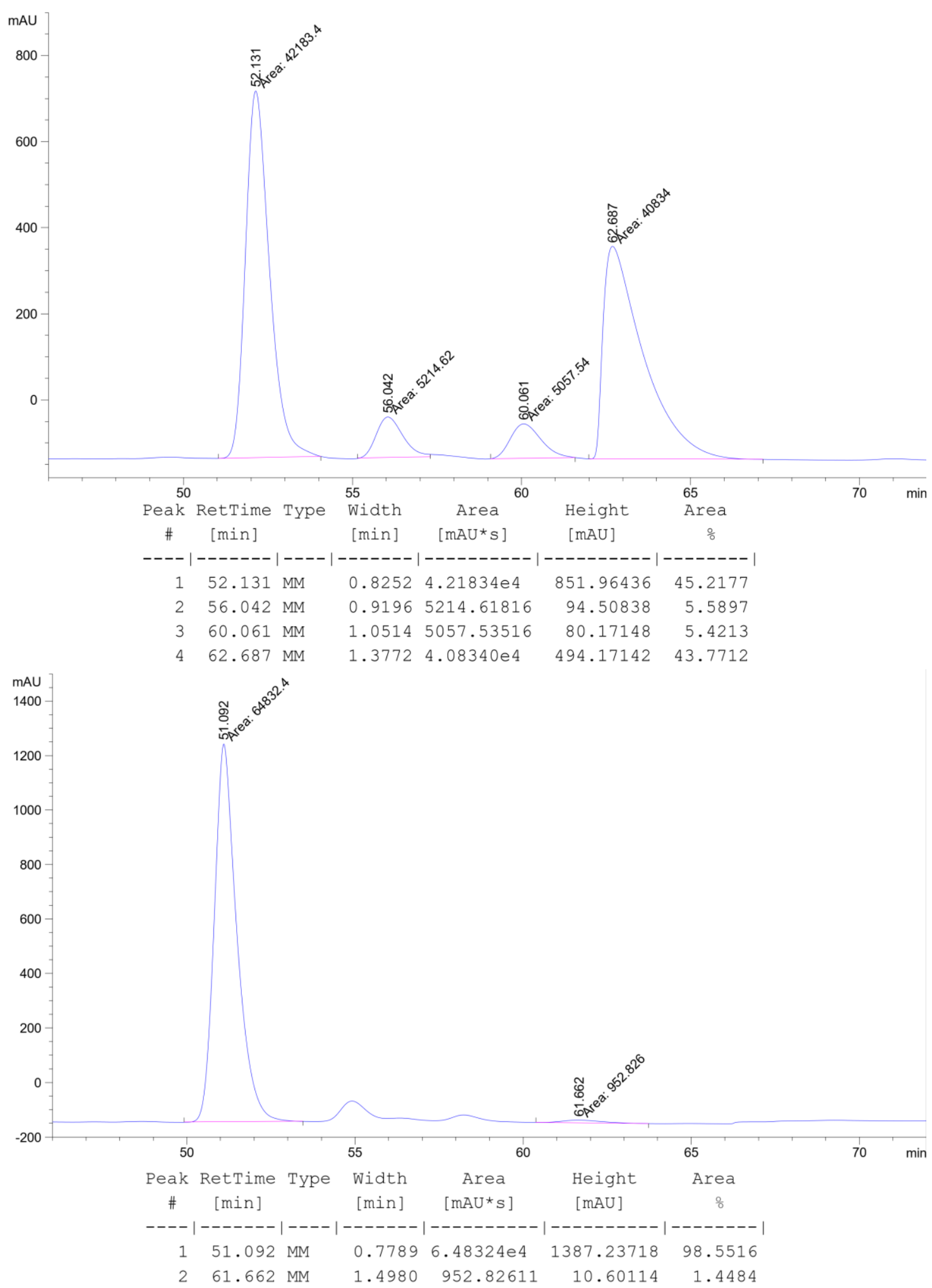
(2S,3S)-3-(benzhydryloxy)-3-methylpent-4-en-2-ol (4r)

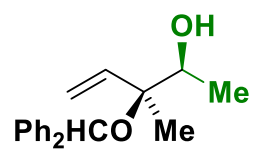

Aldehyde oxidation level: Aldehyde $3 r(44 \mathrm{mg}, 1.0 \mathrm{mmol})$ was subjected to standard reaction conditions $\left(75^{\circ} \mathrm{C}, 24 \mathrm{~h}\right)$. Upon flash column chromatography $\left(\mathrm{SiO}_{2}, 5: 95\right.$ EtOAc:hexanes), the title compound $4 \mathrm{r}$ was obtained as a yellow oil in $77 \%$ yield $(218.6 \mathrm{mg}, 0.154 \mathrm{mmol}, 15: 1 \mathrm{dr}, 87 \%$ ee).

$\operatorname{TLC}\left(\mathrm{SiO}_{2}\right) \mathrm{R}_{\mathrm{f}}=0.20$ (5:95 EtOAc:hexanes)

${ }^{1} \mathbf{H}$ NMR $\left(500 \mathrm{MHz}, \mathrm{CDCl}_{3}\right) \delta: 7.36-7.27(\mathrm{~m}, 4 \mathrm{H}), 7.27-7.22(\mathrm{~m}, 4 \mathrm{H}), 7.23-7.14(\mathrm{~m}, 2 \mathrm{H}), 5.71$ $(\mathrm{dd}, J=17.6,11.0 \mathrm{~Hz}, 1 \mathrm{H}), 5.50(\mathrm{~s}, 1 \mathrm{H}), 5.27-5.20(\mathrm{~m}, 2 \mathrm{H}), 3.79(\mathrm{qd}, J=6.4,2.9 \mathrm{~Hz}, 1 \mathrm{H}), 2.70(\mathrm{~d}$, $J=2.9 \mathrm{~Hz}, 1 \mathrm{H}), 1.11(\mathrm{~d}, J=1.3 \mathrm{~Hz}, 3 \mathrm{H}), 1.06(\mathrm{~d}, J=6.4 \mathrm{~Hz}, 3 \mathrm{H})$.

${ }^{13} \mathrm{C}$ NMR $\left(125 \mathrm{MHz}_{2} \mathrm{CDCl}_{3}\right) \delta: 144.8,144.3,140.5,128.5,128.4,127.2,127.1,126.9,126.7,118.2$, $83.4,76.7,73.4,16.8,15.6$.

HRMS $(\mathrm{Na}+, m / z)$ for $\mathrm{C}_{19} \mathrm{H}_{22} \mathrm{O}_{2}$ : calcd. $=305.1512 ;$ found $=305.1517$.

FTIR (neat): 3442, 3026, 2982, 2358, 1451, 1102, 1020, $697 \mathrm{~cm}^{-1}$

HPLC: (Two connected Chiralcel columns AD-H and OD-H, Hexane:2-PrOH = 99:01, $0.5 \mathrm{~mL} / \mathrm{min}$, $210 \mathrm{~nm}$ )

$[\alpha]_{D}^{24}=+13.7^{\circ}\left(\mathrm{c}=0.19, \mathrm{CHCl}_{3}\right)$. 


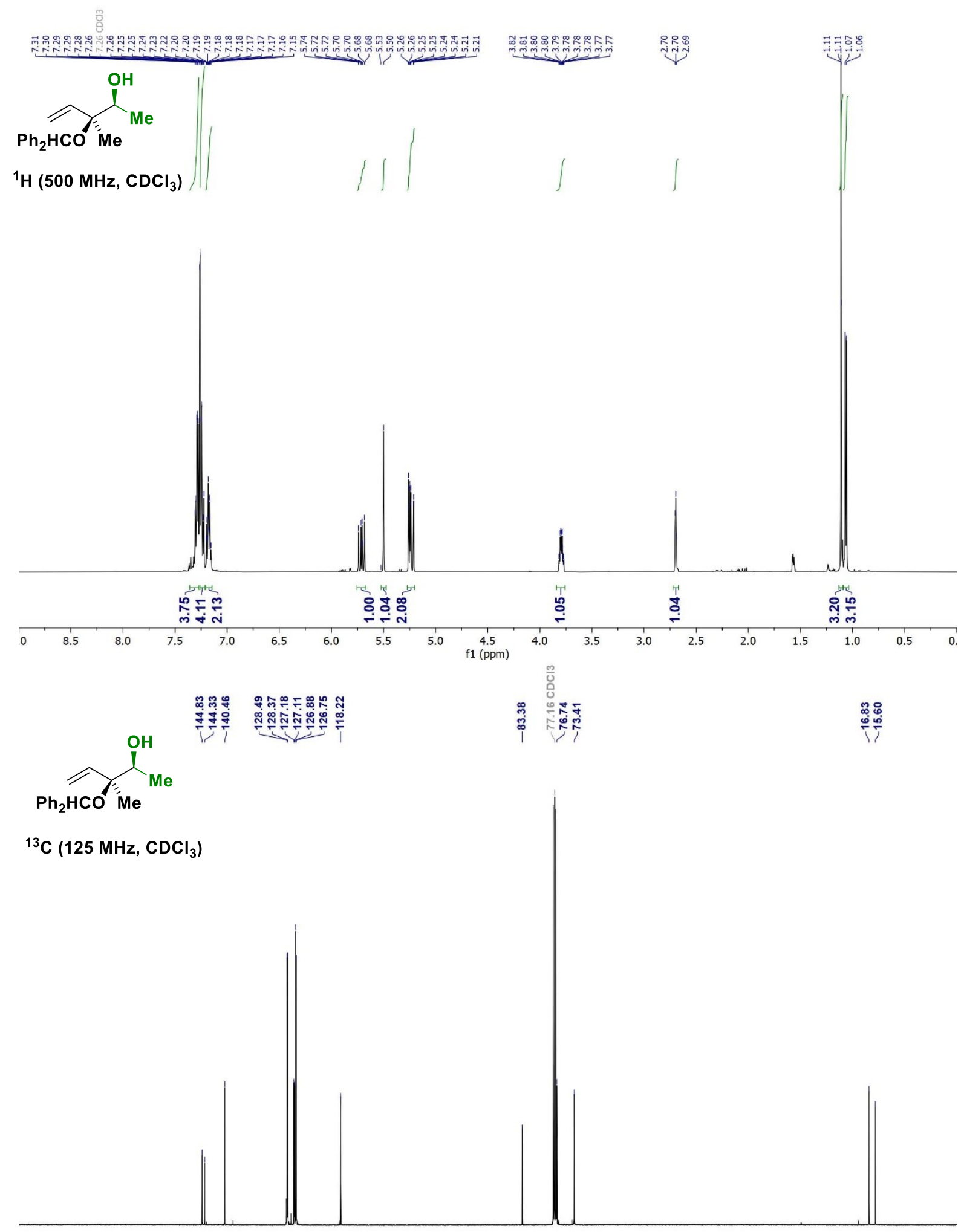

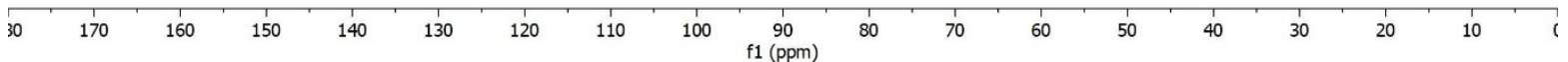



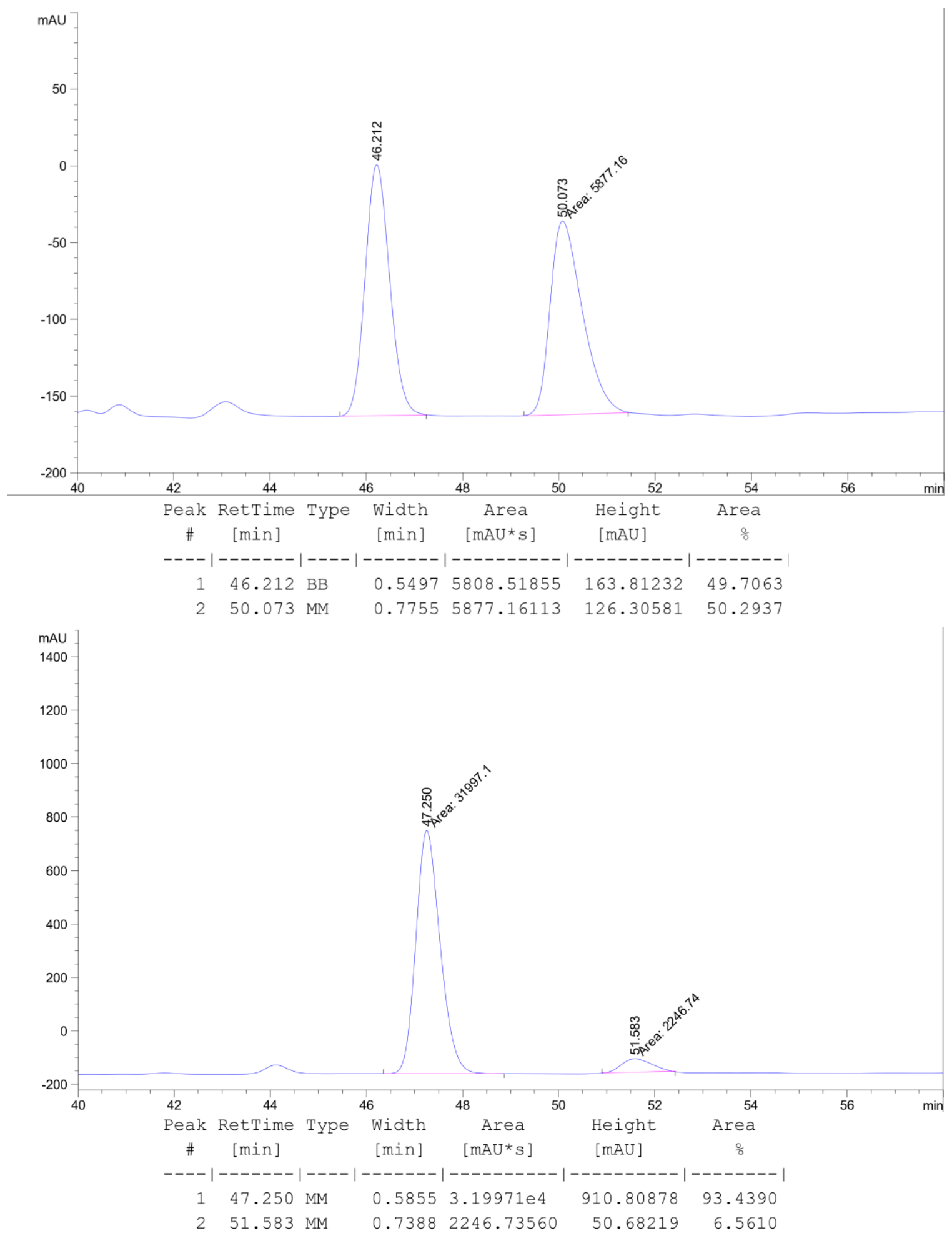


\section{(3S,4S)-4-(benzhydryloxy)-4-methylhex-5-en-3-ol (4s)}

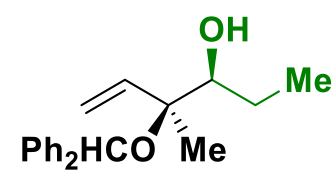

Aldehyde oxidation level: Aldehyde $3 \mathrm{~s}(11.6 \mathrm{mg}, 0.2 \mathrm{mmol})$ was subjected to standard reaction conditions $\left(75^{\circ} \mathrm{C}, 24 \mathrm{~h}\right)$. Upon flash column chromatography $\left(\mathrm{SiO}_{2}, 5: 95 \mathrm{EtOAc}\right.$ :hexanes), the title compound $4 \mathrm{~s}$ was obtained as a yellow oil in $71 \%$ yield $(42.2 \mathrm{mg}, 0.142 \mathrm{mmol}, 13: 1 \mathrm{dr}, 98 \%$ ee).

$\operatorname{TLC}\left(\mathrm{SiO}_{2}\right) \mathrm{R}_{\mathrm{f}}=0.5$ (10:90 EtOAc:hexanes)

${ }^{1} \mathrm{H}$ NMR $\left(500 \mathrm{MHz}, \mathrm{CDCl}_{3}\right) \delta: 7.26(\mathrm{~s}, 2 \mathrm{H}), 7.26-7.19(\mathrm{~m}, 6 \mathrm{H}), 7.19-7.13(\mathrm{~m}, 2 \mathrm{H}), 5.70(\mathrm{dd}, J=$ 17.6, $11.0 \mathrm{~Hz}, 1 \mathrm{H}), 5.49(\mathrm{~s}, 1 \mathrm{H}), 5.23(\mathrm{dd}, J=11.0,1.2 \mathrm{~Hz}, 1 \mathrm{H}), 5.21(\mathrm{dd}, J=17.5,1.2 \mathrm{~Hz}, 1 \mathrm{H}), 3.47$ (dd, $J=10.3,2.1 \mathrm{~Hz}, 1 \mathrm{H}), 2.62(\mathrm{~s}, 1 \mathrm{H}), 1.44(\mathrm{dqd}, J=13.7,7.6,2.1 \mathrm{~Hz}, 1 \mathrm{H}), 1.24$ (ddq, $J=14.2,10.4$, $7.2 \mathrm{~Hz}, 1 \mathrm{H}), 1.09(\mathrm{~s}, 3 \mathrm{H}), 0.98(\mathrm{t}, J=7.4 \mathrm{~Hz}, 3 \mathrm{H})$.

${ }^{13} \mathrm{C} \mathrm{NMR}\left(125 \mathrm{MHz}_{\mathrm{CDCl}}\right)$ ) $: 144.9,144.4,140.6,128.5,128.4,127.2,127.1,126.9,126.7,118.2$, 83.2, 79.3, 76.6, 24.1, 16.1, 11.6.

HRMS $(\mathrm{Na}+, m / z)$ for $\mathrm{C}_{20} \mathrm{H}_{24} \mathrm{O}_{2}$ : calcd. = 319.1669; found $=319.1663$.

FTIR (neat): 3026, 2979, 2875, 1452, 1084, 1023, 740, $697 \mathrm{~cm}^{-1}$

HPLC: (Two connected Chiralcel AD-H columns, Hexane:2-PrOH = 99:01, 0.5 mL/min, $210 \mathrm{~nm}$ ) $[\alpha]_{D}^{24}=+10.0^{\circ}\left(\mathrm{c}=1.0, \mathrm{CHCl}_{3}\right)$ 


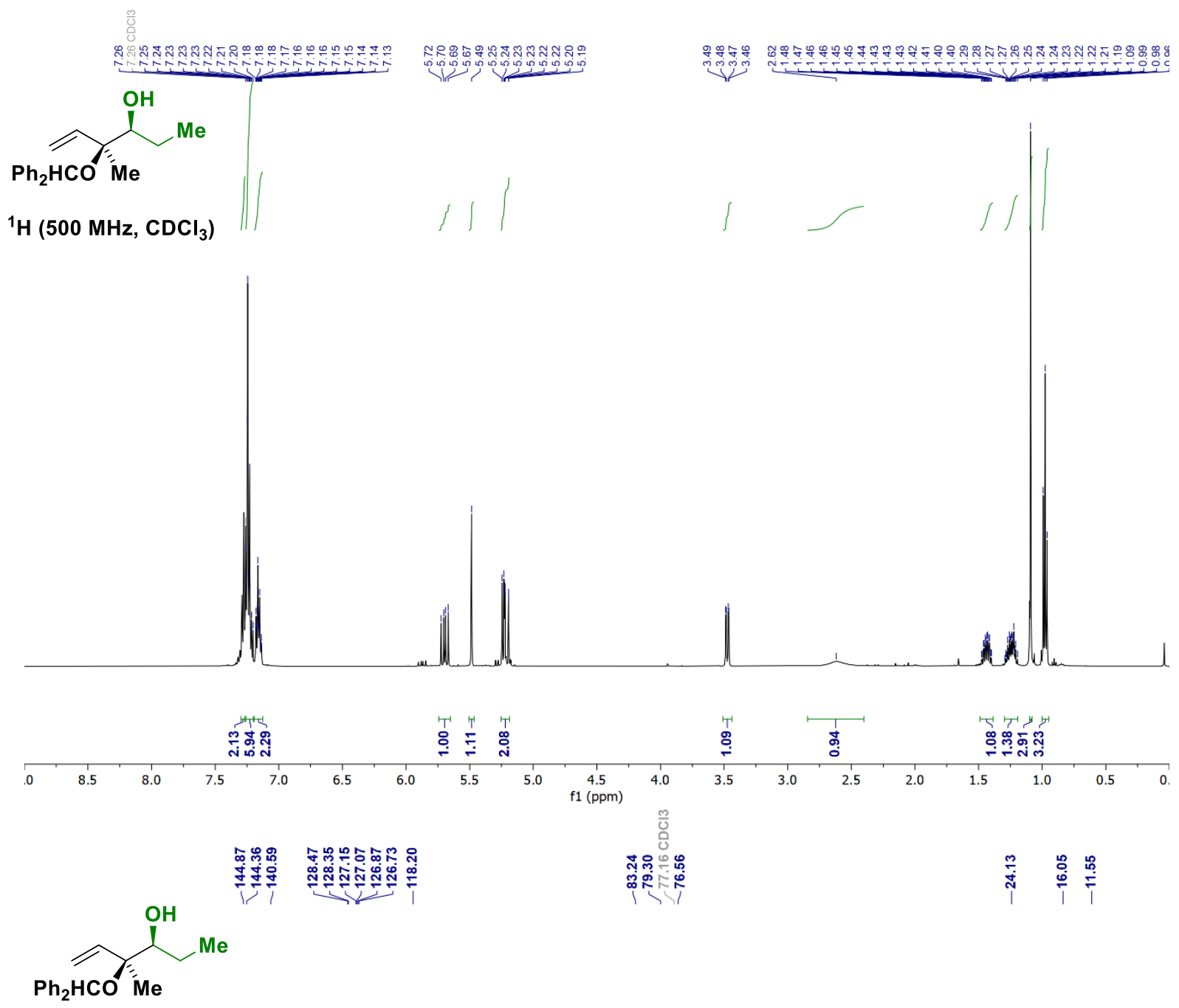

${ }^{13} \mathrm{C}\left(125 \mathrm{MHz}, \mathrm{CDCl}_{3}\right)$

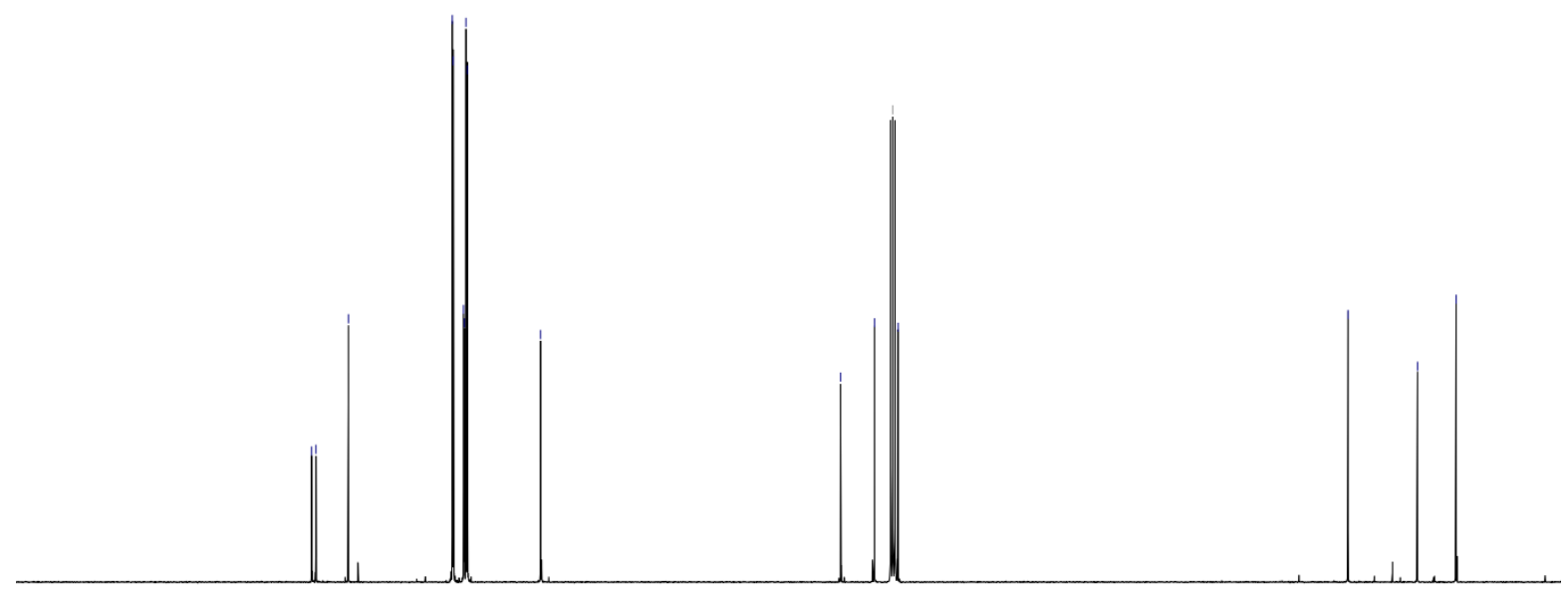




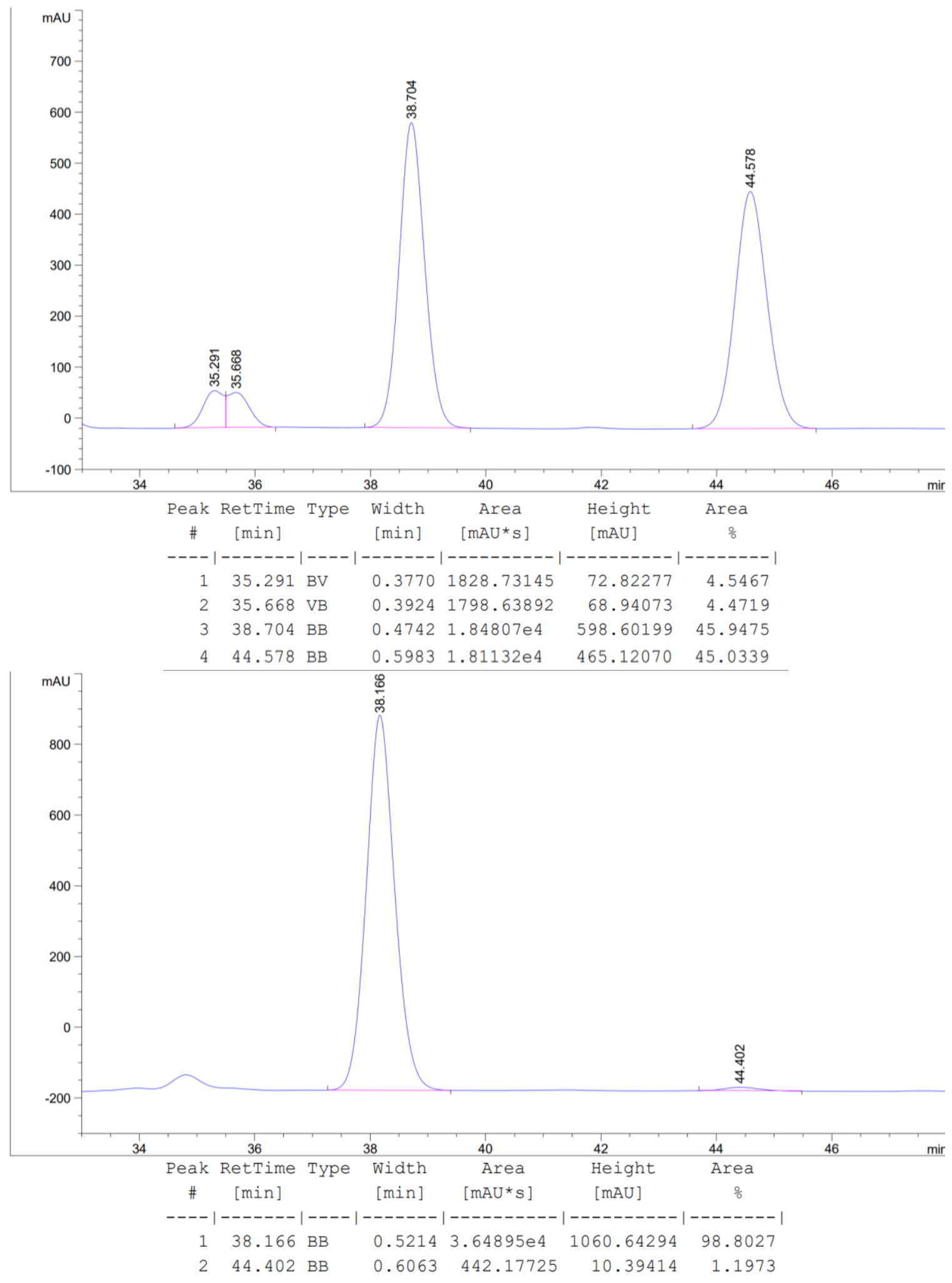


(3S,4S)-3-(benzhydryloxy)-3,6-dimethylhept-1-en-4-ol (4t)

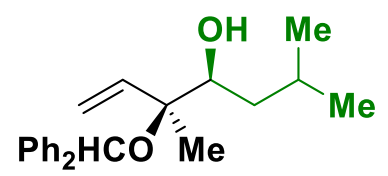

Aldehyde oxidation level: Aldehyde $3 \mathrm{t}(17.2 \mathrm{mg}, 0.2 \mathrm{mmol})$ was subjected to standard reaction conditions (10 mol\% catalyst loading, $75{ }^{\circ} \mathrm{C}, \mathbf{2 4} \mathrm{h}$ ). Upon flash column chromatography $\left(\mathrm{SiO}_{2}\right.$, 3:97 EtOAc:hexanes), the title compound $\mathbf{4 t}$ was obtained as a yellow oil in $65 \%$ yield $(42.2 \mathrm{mg}$, $0.13 \mathrm{mmol}, 8: 1 \mathrm{dr}, 97 \% \mathrm{ee})$.

$\operatorname{TLC}\left(\mathrm{SiO}_{2}\right) \mathrm{R}_{\mathrm{f}}=0.3$ (05:95 EtOAc:hexanes)

${ }^{1} \mathbf{H}$ NMR $\left(500 \mathrm{MHz}, \mathrm{CDCl}_{3}\right)$ 8: $7.35-7.27(\mathrm{~m}, 8 \mathrm{H}), 7.24-7.18(\mathrm{~m}, 2 \mathrm{H}), 5.71(\mathrm{dd}, \mathrm{J}=17.6,11.0 \mathrm{~Hz}$, $1 \mathrm{H}), 5.53(\mathrm{~s}, 1 \mathrm{H}), 5.31-5.23(\mathrm{~m}, 2 \mathrm{H}), 3.69(\mathrm{dd}, \mathrm{J}=10.7,2.2 \mathrm{~Hz}, 1 \mathrm{H}), 2.70(\mathrm{~s}, 1 \mathrm{H}), 1.85(\mathrm{ddq}, \mathrm{J}=$ 9.4, 6.6, $4.1 \mathrm{~Hz}, 1 \mathrm{H}), 1.33-1.24(\mathrm{~m}, 1 \mathrm{H}), 1.13(\mathrm{~s}, 3 \mathrm{H}), 1.12-1.07(\mathrm{~m}, 1 \mathrm{H}), 0.94(\mathrm{~d}, \mathrm{~J}=6.7 \mathrm{~Hz}, 3 \mathrm{H})$, $0.89(\mathrm{~d}, \mathrm{~J}=6.5 \mathrm{~Hz}, 3 \mathrm{H})$.

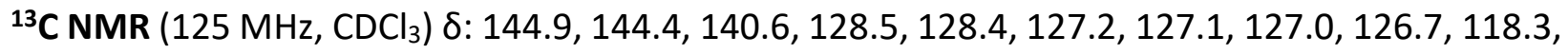
83.4, 76.6, 75.6, 40.0, 25.2, 24.1, 21.7, 15.8 .

HRMS $(\mathrm{Na}+, m / z)$ for $\mathrm{C}_{22} \mathrm{H}_{28} \mathrm{O}_{2}$ : calcd. $=347.1982 ;$ found $=347.1989$.

FTIR (neat): 2953, 72868, 1493, 1453, 1024, 922, 470, $699 \mathrm{~cm}^{-1}$

HPLC: (Two connected Chiralcel AD-H columns, Hexane:2-PrOH =99:01, $1.0 \mathrm{~mL} / \mathrm{min}, 230 \mathrm{~nm}$ ) $[\alpha]_{D}^{24}=+23.0^{\circ}\left(\mathrm{c}=1.0, \mathrm{CHCl}_{3}\right)$ 

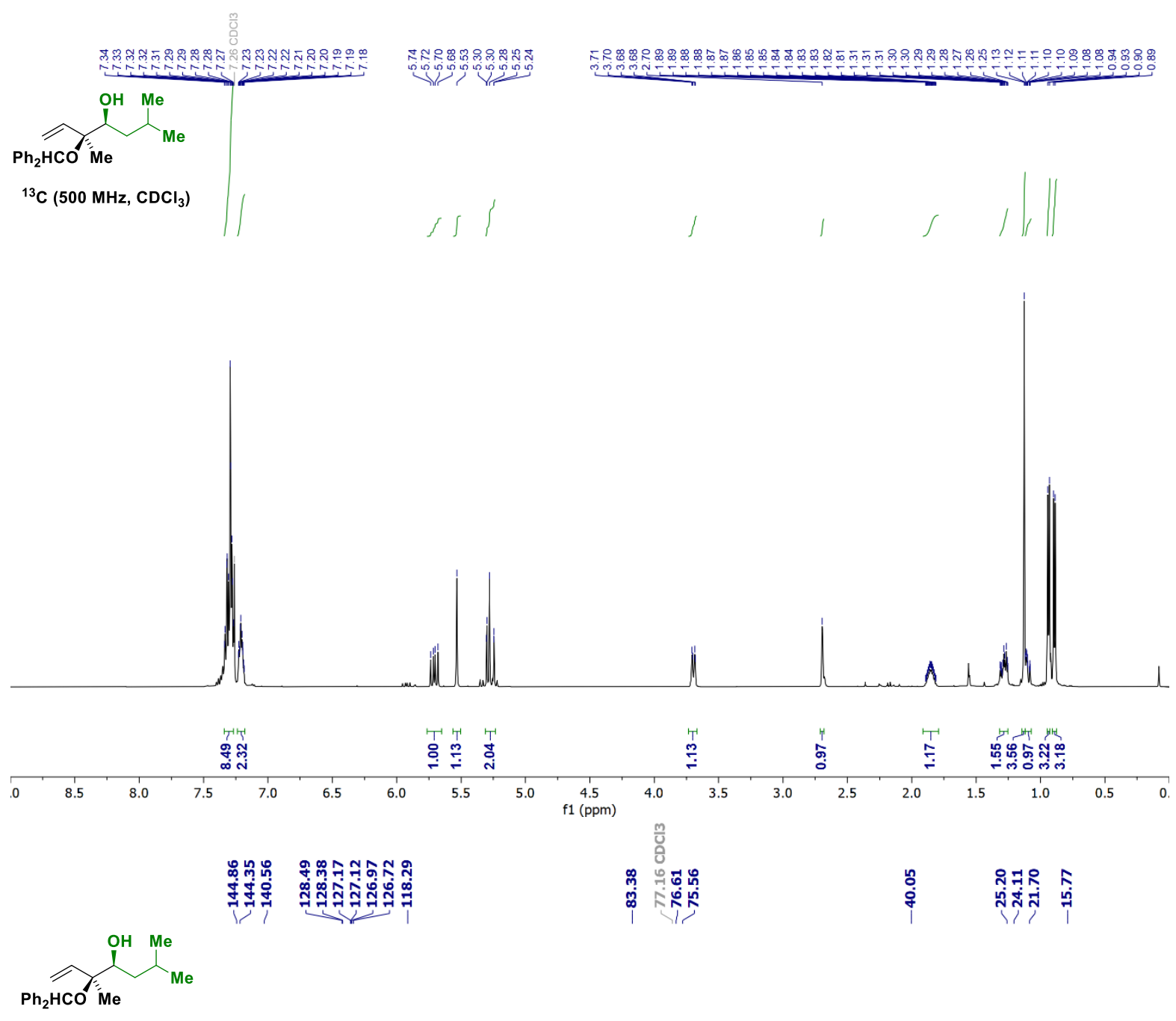

${ }^{13} \mathrm{C}\left(125 \mathrm{MHz}, \mathrm{CDCl}_{3}\right)$

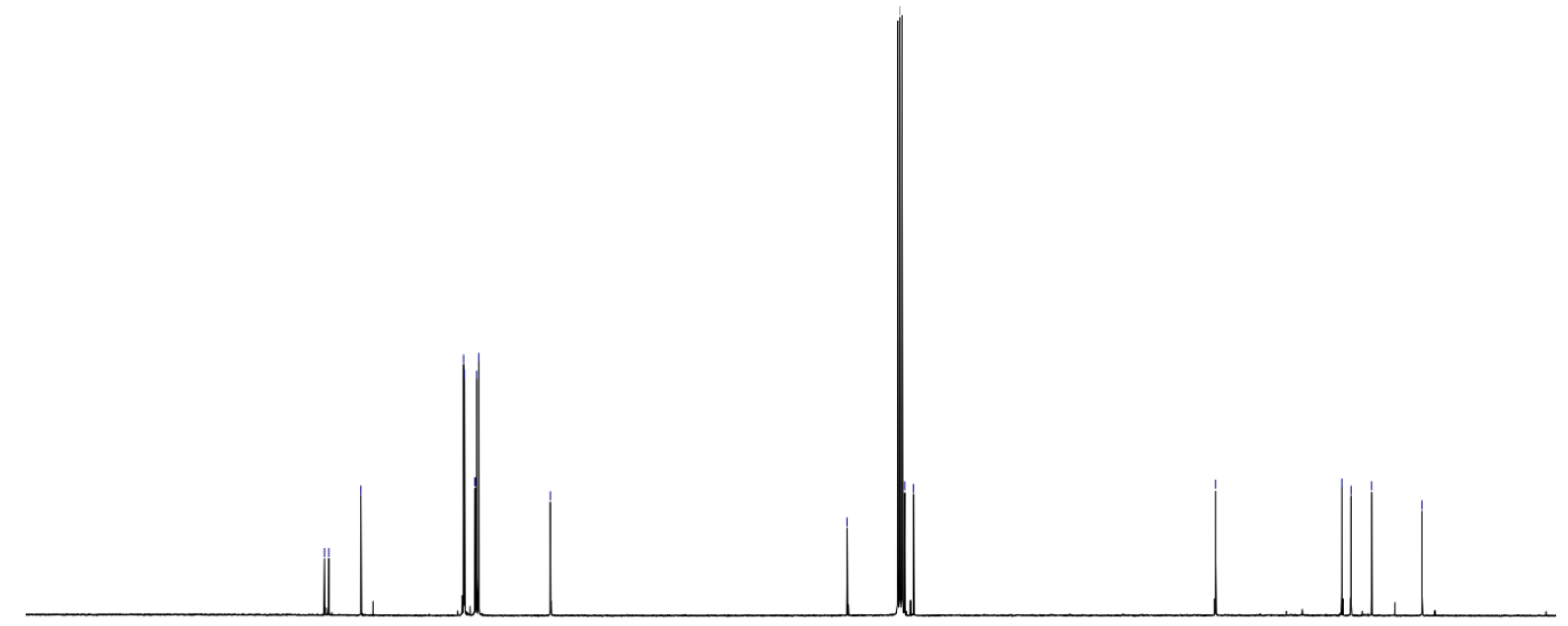

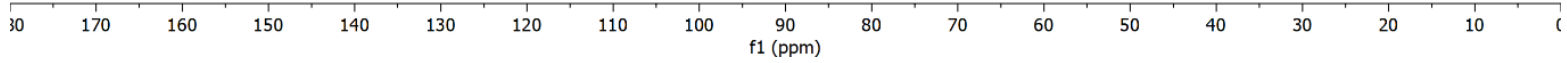



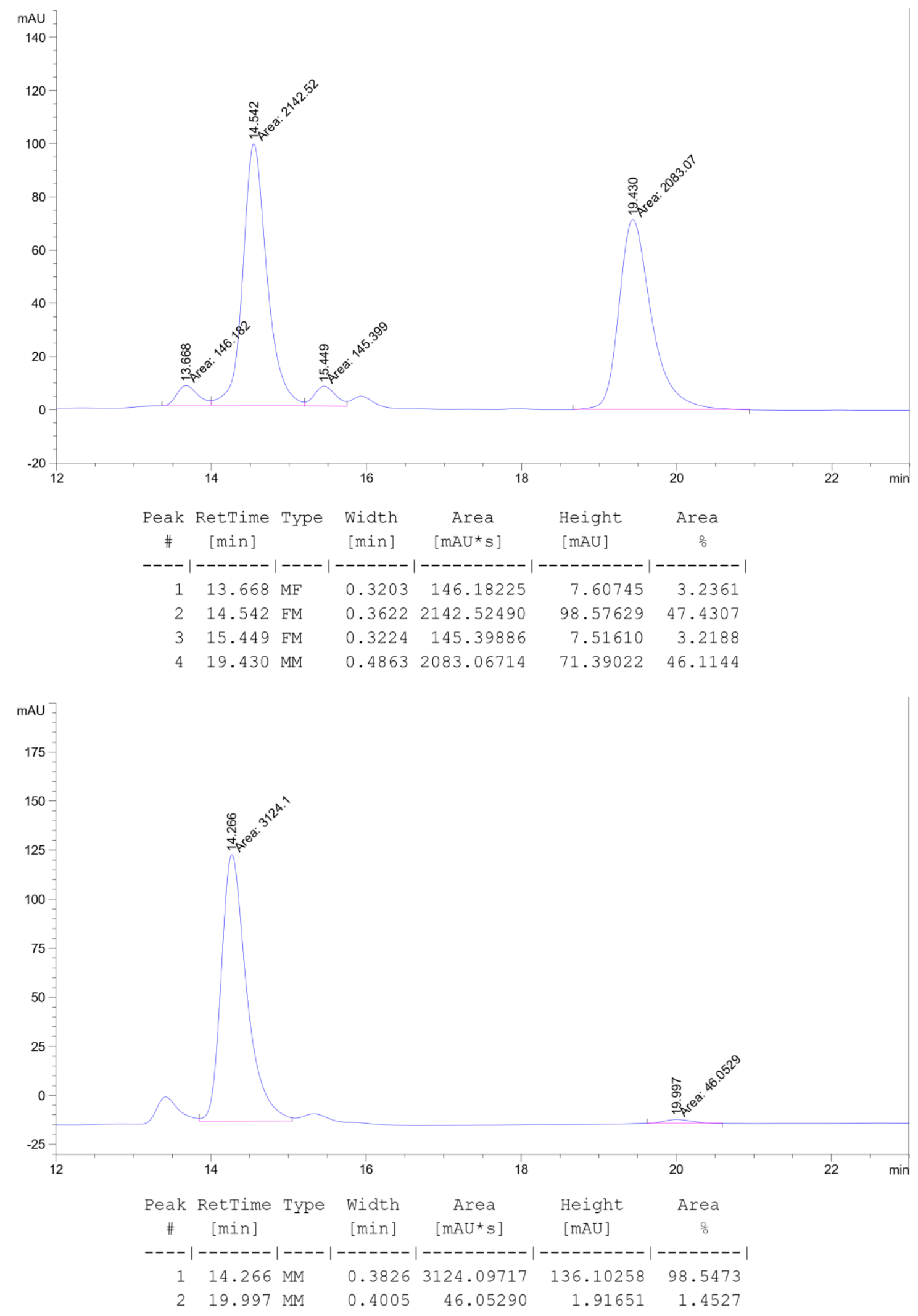
tert-butyl 3-((2S,3S)-3-(benzhydryloxy)-2-hydroxy-3-methylpent-4-en-1-yl)azetidine-1carboxylate $(4 \mathrm{u})$

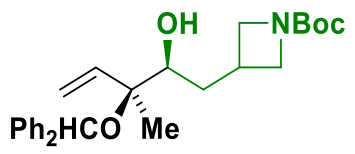

Aldehyde oxidation level: Aldehyde $3 \mathbf{u}(40.0 \mathrm{mg}, 0.2 \mathrm{mmol})$ was subjected to standard reaction conditions ( 10 mol\% catalyst loading, $75{ }^{\circ} \mathrm{C}, \mathbf{4 8} \mathrm{h}$ ). Upon flash column chromatography $\left(\mathrm{SiO}_{2}\right.$, 15:85 EtOAc:hexanes), the title compound $4 \mathrm{u}$ was obtained as a yellow oil in $62 \%$ yield ( $54.2 \mathrm{mg}$, $0.124 \mathrm{mmol}, 11: 1 \mathrm{dr}, 96 \%$ ee).

$\operatorname{TLC}\left(\mathrm{SiO}_{2}\right) \mathrm{R}_{\mathrm{f}}=0.25$ (20:80 EtOAc:hexanes)

${ }^{1} \mathbf{H}$ NMR $\left(500 \mathrm{MHz} \mathrm{CDCl}_{3}\right) \delta: 7.33-7.29(\mathrm{~m}, 4 \mathrm{H}), 7.28-7.26(\mathrm{~m}, 4 \mathrm{H}), 7.24-7.18(\mathrm{~m}, 2 \mathrm{H}), 5.70$ (dd, $J=17.6,10.9 \mathrm{~Hz}, 1 \mathrm{H}), 5.52(\mathrm{~s}, 1 \mathrm{H}), 5.34-5.26(\mathrm{~m}, 2 \mathrm{H}), 4.02(\mathrm{dt}, J=14.8,8.4 \mathrm{~Hz}, 2 \mathrm{H}), 3.60$ $(\mathrm{dd}, J=8.6,5.6 \mathrm{~Hz}, 1 \mathrm{H}), 3.55-3.49(\mathrm{~m}, 2 \mathrm{H}), 2.76(\mathrm{dq}, J=13.5,7.0 \mathrm{~Hz}, 1 \mathrm{H}), 2.68(\mathrm{~s}, 1 \mathrm{H}), 1.68-$ $1.58(\mathrm{~m}, 2 \mathrm{H}), 1.43(\mathrm{~s}, 12 \mathrm{H}), 1.13(\mathrm{~s}, 3 \mathrm{H})$.

${ }^{13} \mathrm{C}$ NMR $\left(125 \mathrm{MHz}, \mathrm{CDCl}_{3}\right) \delta: 156.6,144.6,144.1,140.1,128.5,128.4,127.3,127.2,126.9,126.7$, $118.9,82.9,79.3,76.7,76.2,35.6,29.8,28.6,26.9,15.8$.

HRMS $(\mathrm{Na}+, m / z)$ for $\mathrm{C}_{27} \mathrm{H}_{35} \mathrm{NO}_{4}$ : calcd. $=460.2458 ;$ found $=460.2471$.

FTIR (neat): 2988, 2360, 1685, 1415, 1263, 1143, $748 \mathrm{~cm}^{-1}$

HPLC: (Two connected Chiralcel AD-H columns, Hexane:2-PrOH =98:02, $1.0 \mathrm{~mL} / \mathrm{min}, 210 \mathrm{~nm}$ ) $[\alpha]_{D}^{24}=+30.8^{\circ}\left(\mathrm{c}=0.13, \mathrm{CHCl}_{3}\right)$. 

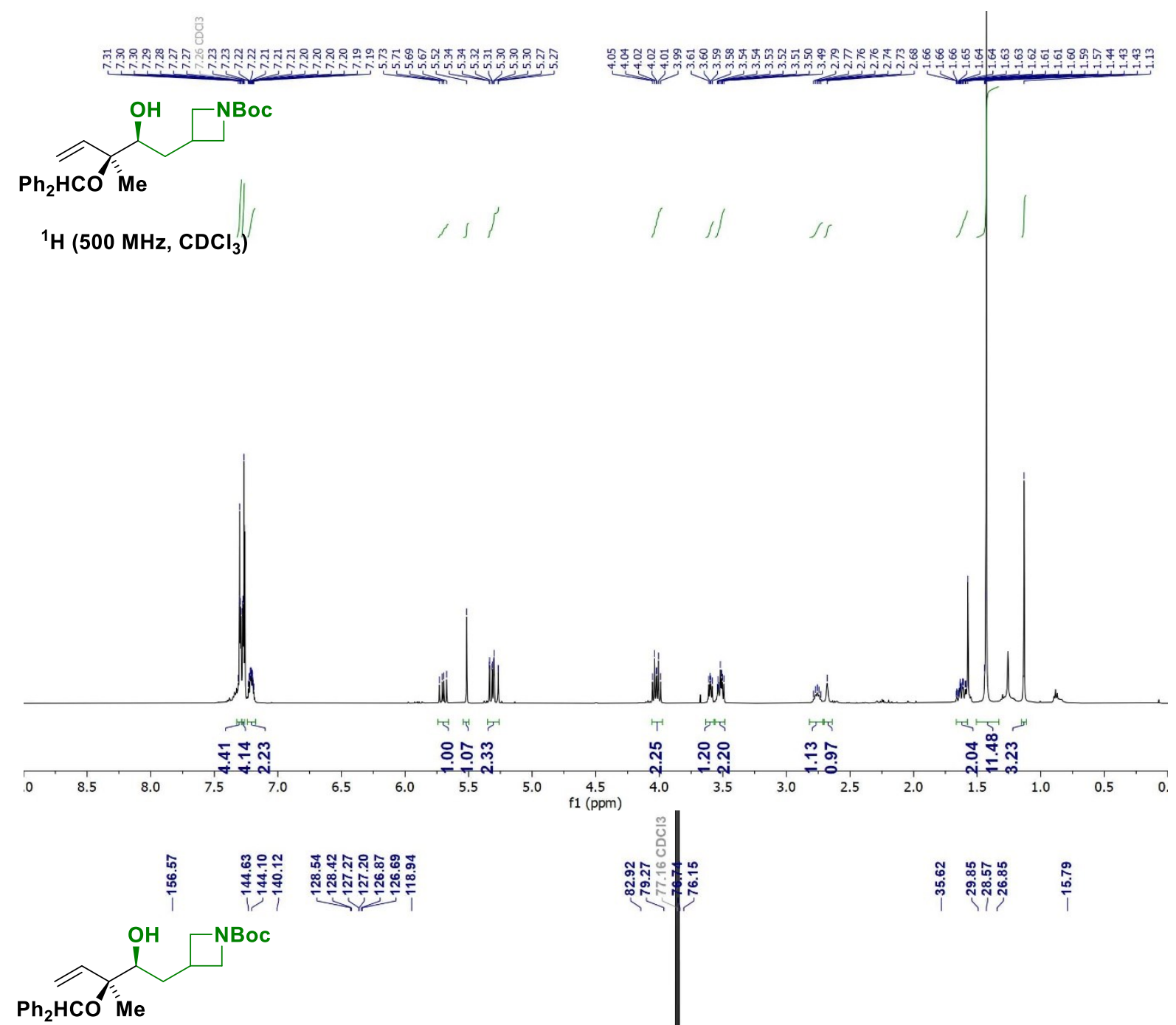

${ }^{13} \mathrm{C}\left(125 \mathrm{MHz}, \mathrm{CDCl}_{3}\right)$

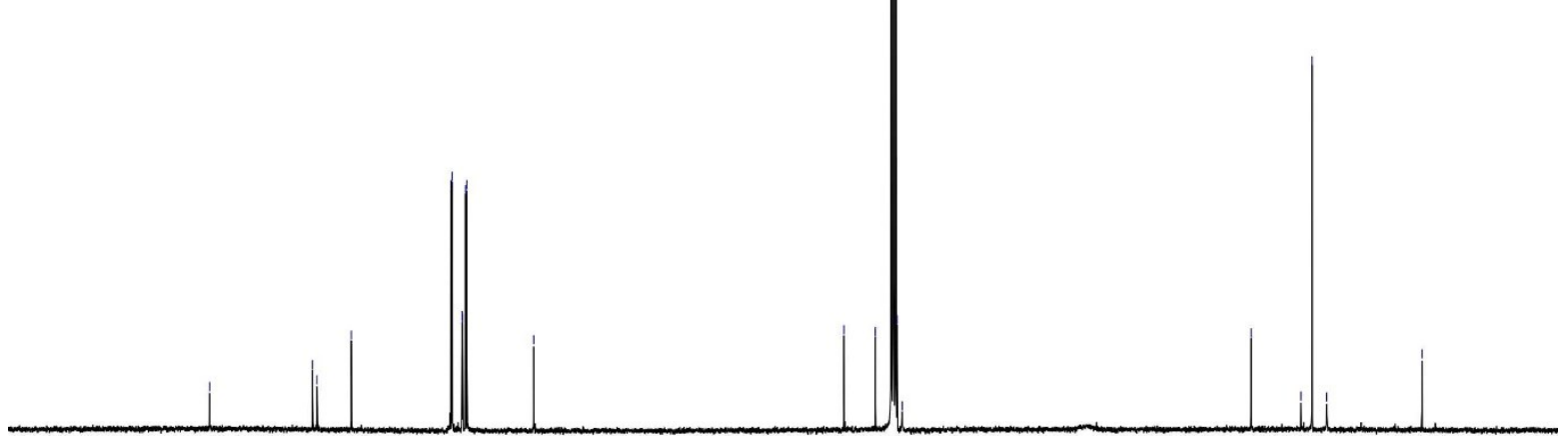

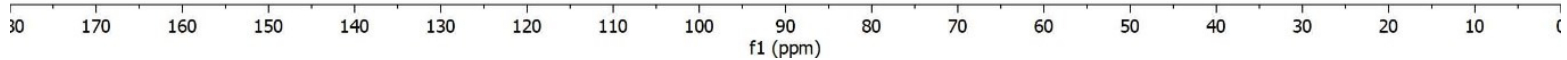




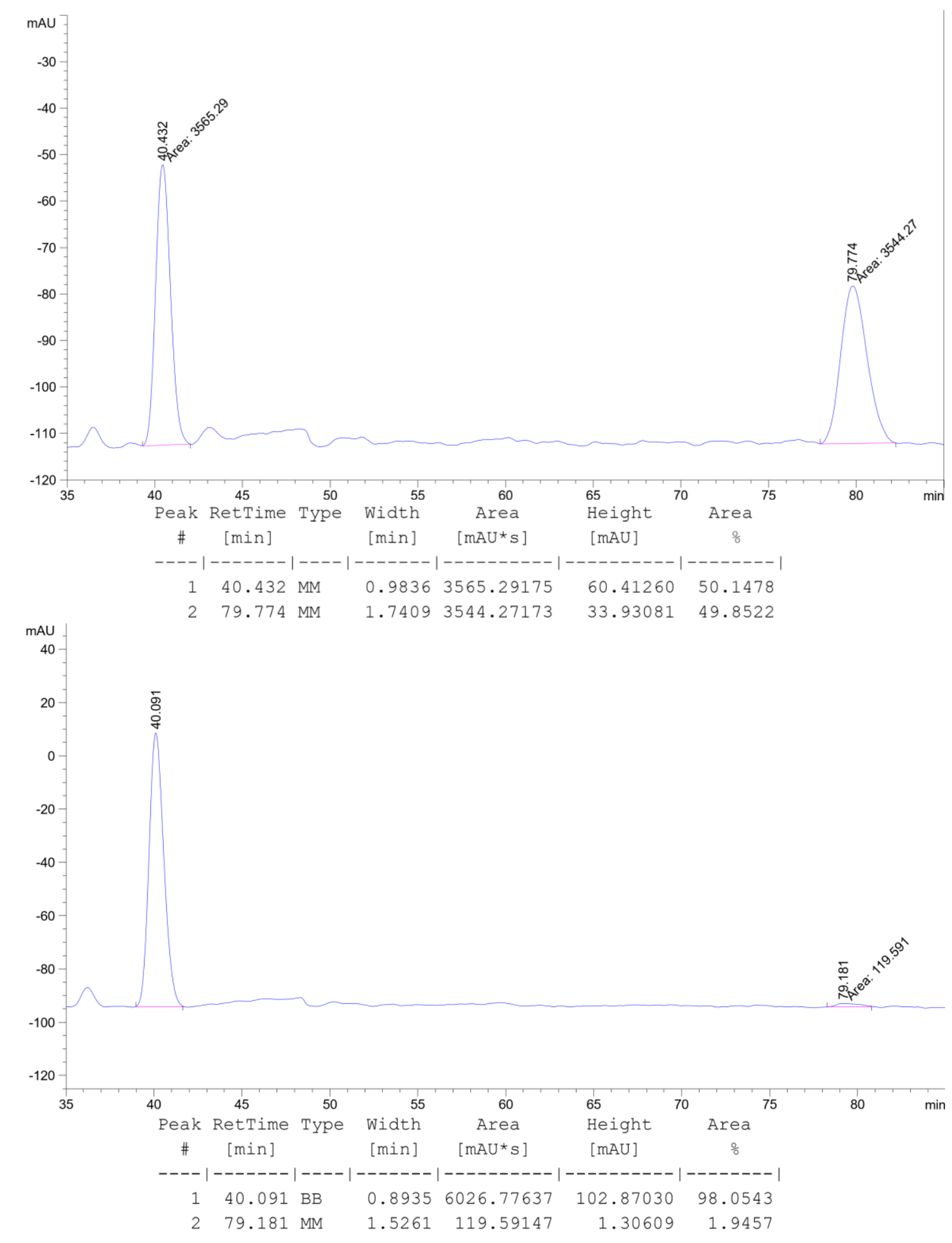


tert-butyl 4-((2S,3S)-3-(benzhydryloxy)-2-hydroxy-3-methylpent-4-en-1-yl)piperidine-1carboxylate (4v)

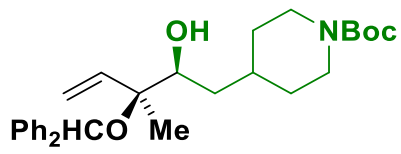

Aldehyde oxidation level: Aldehyde $3 \mathbf{v}(40.0 \mathrm{mg}, 0.2 \mathrm{mmol})$ was subjected to standard reaction conditions ( 10 mol\% catalyst loading, $75{ }^{\circ} \mathrm{C}, \mathbf{4 8} \mathrm{h}$ ). Upon flash column chromatography $\left(\mathrm{SiO}_{2}\right.$, 10:90 EtOAc:hexanes), the title compound $\mathbf{4 v}$ was obtained as a yellow oil in $60 \%$ yield ( $55.5 \mathrm{mg}$, $0.12 \mathrm{mmol}, 10: 1 \mathrm{dr}, 99 \%$ ee).

$\operatorname{TLC}\left(\mathrm{SiO}_{2}\right) \mathrm{R}_{\mathrm{f}}=0.35$ (20:80 EtOAc:hexanes)

${ }^{1} \mathbf{H}$ NMR $\left(500 \mathrm{MHz} \mathrm{CDCl}_{3}\right) \delta: 7.32-7.25(\mathrm{~m}, 5 \mathrm{H}), 7.30-7.23(\mathrm{~m}, 3 \mathrm{H}) 7.23-7.16(\mathrm{~m}, 2 \mathrm{H}), 5.67$ $(\mathrm{dd}, J=17.6,10.9 \mathrm{~Hz}, 1 \mathrm{H}), 5.51(\mathrm{~s}, 1 \mathrm{H}), 5.30-5.22(\mathrm{~m}, 2 \mathrm{H}), 4.05(\mathrm{~s}, 2 \mathrm{H}), 3.71(\mathrm{dt}, J=10.4,2.0 \mathrm{~Hz}$, $1 \mathrm{H}), 2.72(\mathrm{~s}, 1 \mathrm{H}), 2.67(\mathrm{~d}, J=17.4 \mathrm{~Hz}, 2 \mathrm{H}), 1.83-1.72(\mathrm{~m}, 1 \mathrm{H}), 1.71-1.56(\mathrm{~m}, 2 \mathrm{H}), 1.44(\mathrm{~s}, 9 \mathrm{H})$, $1.33-1.23(\mathrm{~m}, 1 \mathrm{H}), 1.22-1.12(\mathrm{~m}, 2 \mathrm{H}), 1.11(\mathrm{~s}, 3 \mathrm{H}), 1.03-0.92(\mathrm{~m}, 1 \mathrm{H})$.

${ }^{13} \mathrm{C}$ NMR $\left(125 \mathrm{MHz}, \mathrm{CDCl}_{3}\right) \delta: 155.0,144.7,144.2,140.3,128.5,128.4,127.2,127.2,126.9,126.7$, $118.6,83.2,79.3,76.7,74.7,37.7,33.5,33.1,31.5,28.6,15.7$.

HRMS $(\mathrm{Na}+, m / z)$ for $\mathrm{C}_{29} \mathrm{H}_{39} \mathrm{NO}_{4}$ : calcd. $=488.2771 ;$ found $=488.2780$.

FTIR (neat): 3440, 2934, 2632, 2243, 1676, 1427, 1165, 906, $727 \mathrm{~cm}^{-1}$

HPLC: (Two connected Chiralcel columns AD-H and ODH, Hexane:2-PrOH $=96: 04,0.5 \mathrm{~mL} / \mathrm{min}$, $210 \mathrm{~nm})$

$[\alpha]_{D}^{24}=+21.6^{\circ}\left(\mathrm{c}=0.37, \mathrm{CHCl}_{3}\right)$. 


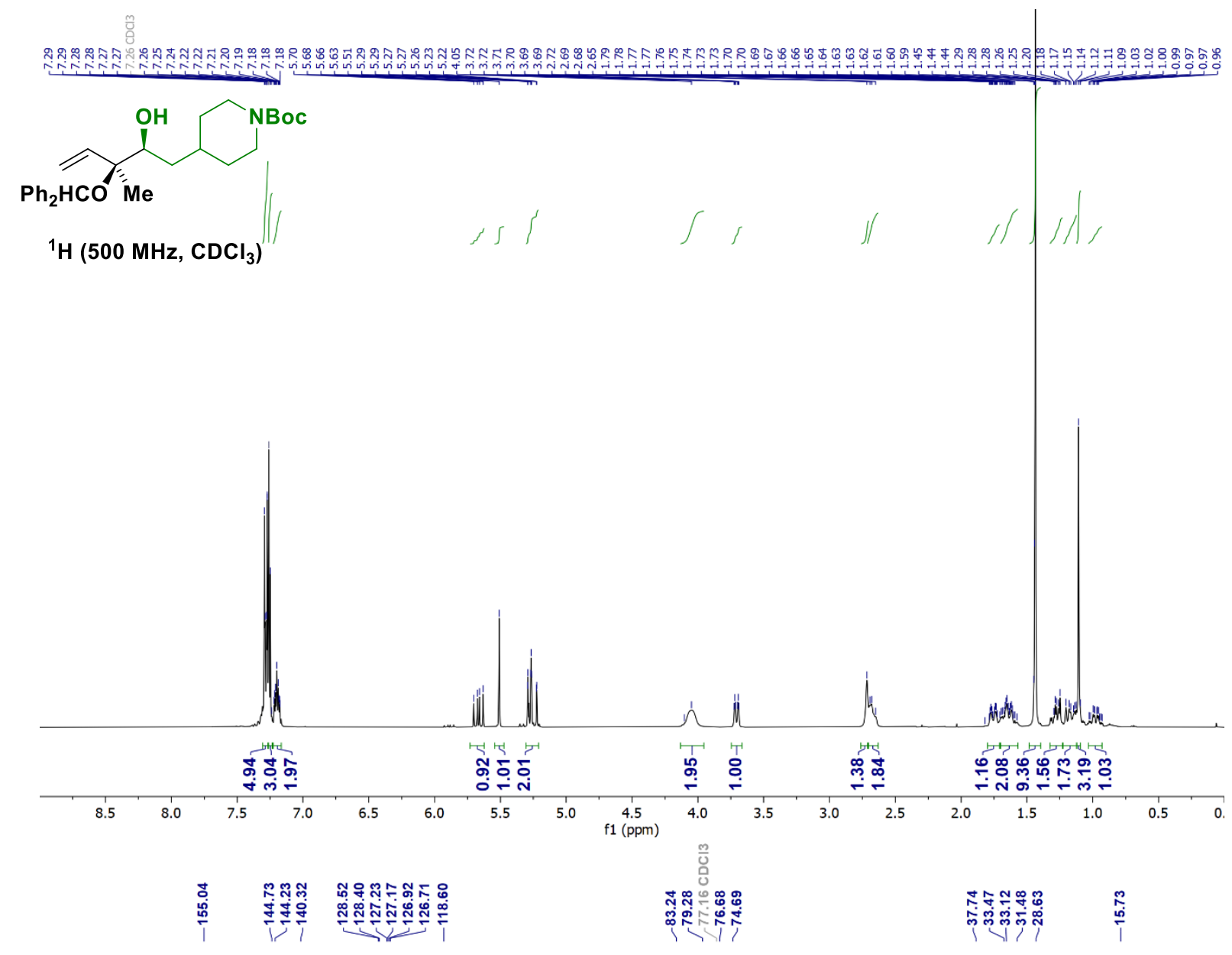

Me

${ }^{13} \mathrm{C}\left(125 \mathrm{MHz}, \mathrm{CDCl}_{3}\right)$

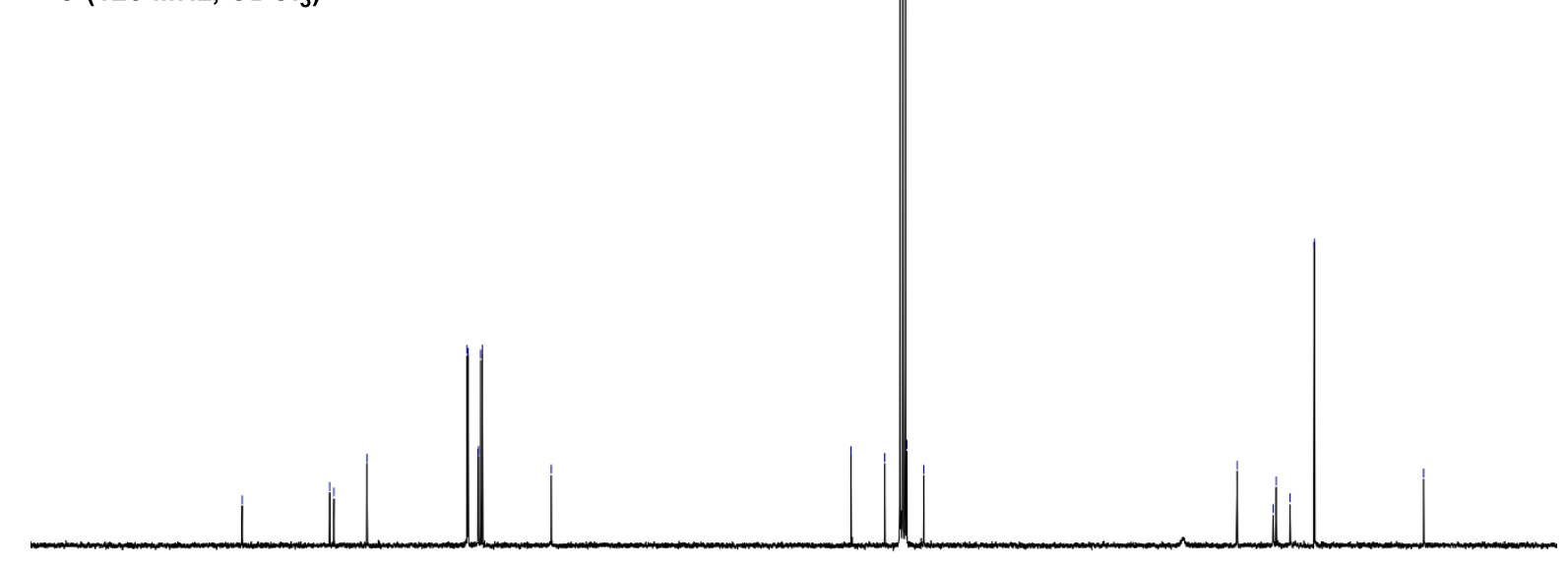

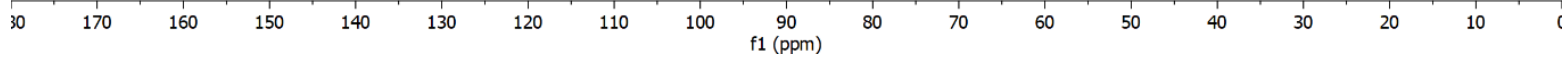




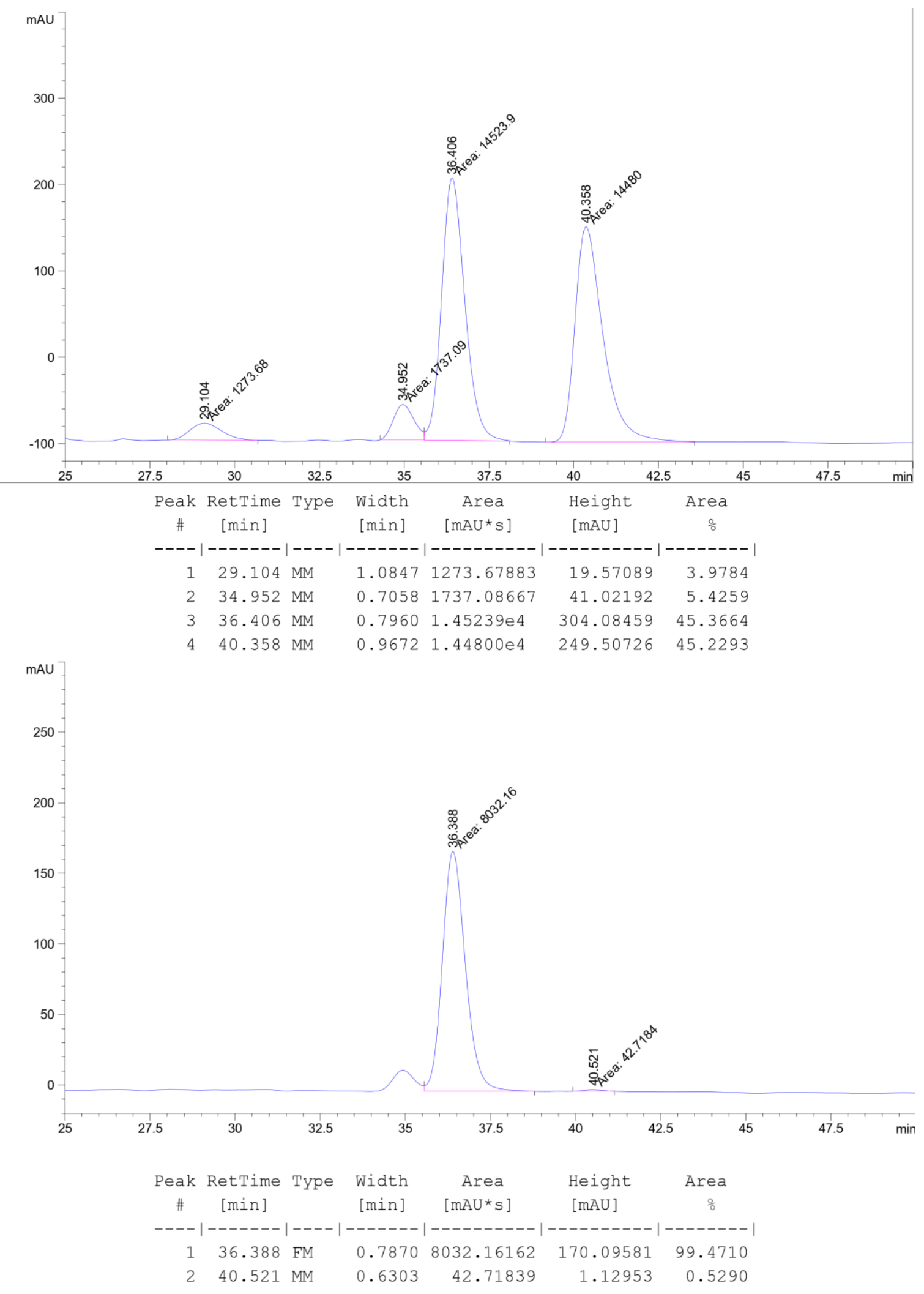


(3S,4S)-4-(benzhydryloxy)-1-((tert-butyldimethylsilyl)oxy)-4-methylhex-5-en-3-ol (4w)

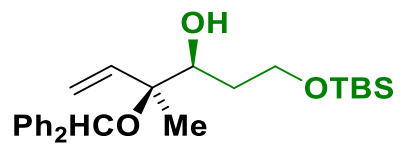

Aldehyde oxidation level: Aldehyde $3 w(37.6 \mathrm{mg}, 0.2 \mathrm{mmol})$ was subjected to standard reaction conditions $\left(75^{\circ} \mathrm{C}, 24 \mathrm{~h}\right.$ ). Upon flash column chromatography $\left(\mathrm{SiO}_{2}, 2: 98 \mathrm{EtOAc}\right.$ :hexanes), the title compound $\mathbf{4 w}$ was obtained as a colorless oil in $64 \%$ yield $(54.6 \mathrm{mg}, 0.128 \mathrm{mmol}, 15: 1 \mathrm{dr}, 94 \%$ ee).

$\operatorname{TLC}\left(\mathrm{SiO}_{2}\right) \mathrm{R}_{\mathrm{f}}=0.15$ (1:99 EtOAc:hexanes)

${ }^{1} \mathbf{H}$ NMR $\left(400 \mathrm{MHz}, \mathrm{CDCl}_{3}\right) \delta: 7.36-7.26(\mathrm{~m}, 8 \mathrm{H}), 7.24-7.17(\mathrm{~m}, 2 \mathrm{H}), 5.77(\mathrm{dd}, \mathrm{J}=17.1,11.5 \mathrm{~Hz}$, 1H), $5.55(\mathrm{~s}, 1 \mathrm{H}), 5.30(\mathrm{~d}, \mathrm{~J}=0.5 \mathrm{~Hz}, 1 \mathrm{H}), 5.26(\mathrm{dd}, \mathrm{J}=7.6,1.3 \mathrm{~Hz}, 1 \mathrm{H}), 3.85-3.75(\mathrm{~m}, 3 \mathrm{H}), 3.01$ $(\mathrm{dd}, \mathrm{J}=2.5,1.0 \mathrm{~Hz}, 1 \mathrm{H}), 1.81-1.72(\mathrm{~m}, 1 \mathrm{H}), 1.52(\mathrm{ddt}, J=13.9,10.4,5.8 \mathrm{~Hz}, 1 \mathrm{H}), 1.18(\mathrm{~s}, 3 \mathrm{H}), 0.91$ $(\mathrm{s}, 9 \mathrm{H}), 0.07(\mathrm{~d}, \mathrm{~J}=1.1 \mathrm{~Hz}, 6 \mathrm{H})$.

${ }^{13} \mathrm{C}$ NMR $\left(100 \mathrm{MHz}_{2} \mathrm{CDCl}_{3}\right) \delta: 145.0,144.5,140.2,128.4,128.4,127.1,127.0,126.9,126.8,118.0$, 82.7, 76.6, 75.5, 61.7, 34.1, 26.1, 18.5, 17.4, -5.2, -5.2.

HRMS $(\mathrm{Na}+, m / z)$ for $\mathrm{C}_{27} \mathrm{H}_{36} \mathrm{O}_{2}$ : calcd. $=415.2608$; found $=415.2615$.

FTIR (neat): 3450, 2988, 1453, 1265, 1080, 883, $748 \mathrm{~cm}^{-1}$

HPLC: (Two connected Chiralcel columns AD-H and OD-H, Hexane:2-PrOH = 98:02, $0.5 \mathrm{~mL} / \mathrm{min}$, 210nm)

$[\alpha]_{D}^{24}=+14.0^{\circ}\left(\mathrm{c}=0.2, \mathrm{CHCl}_{3}\right)$. 


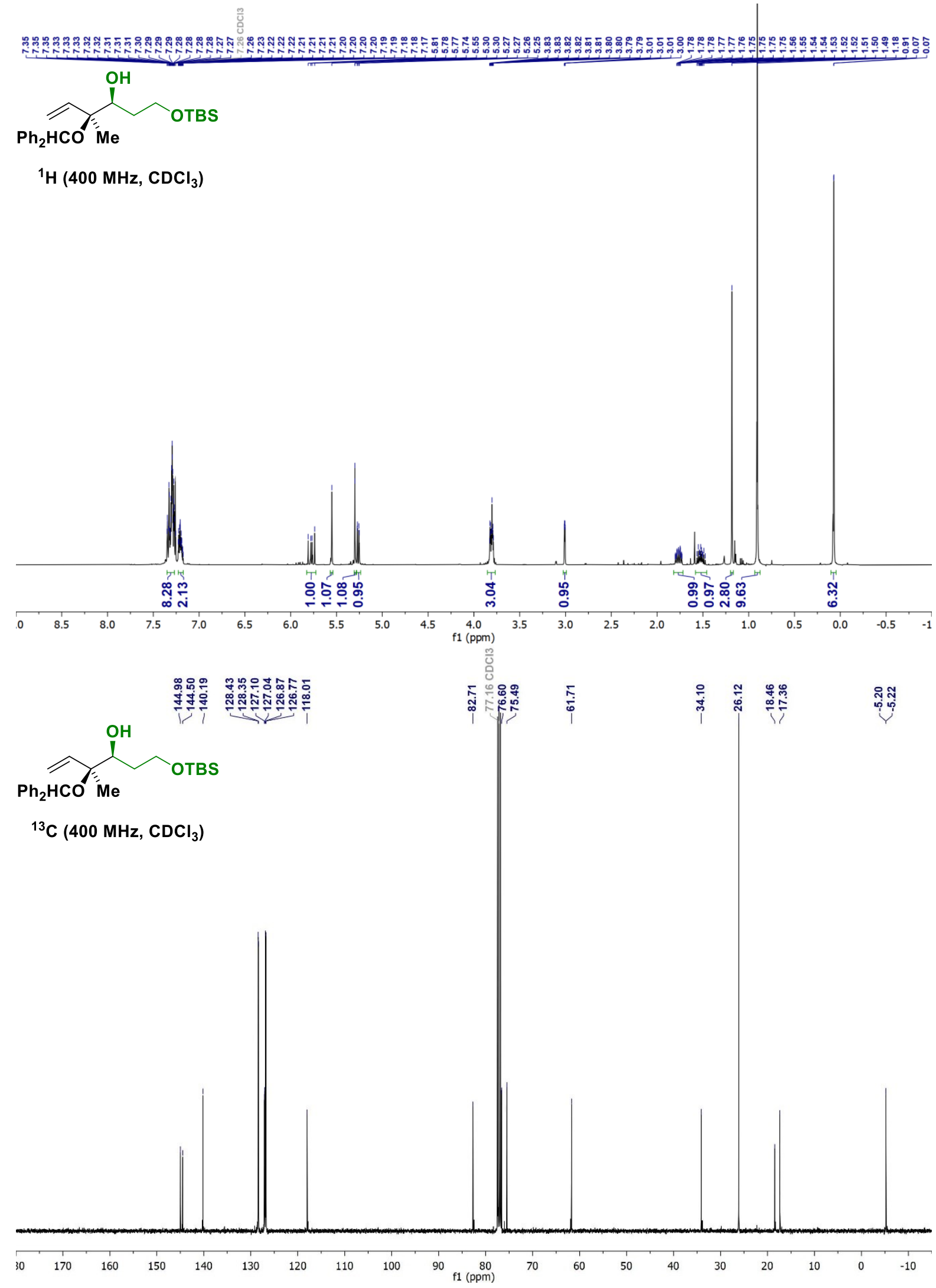



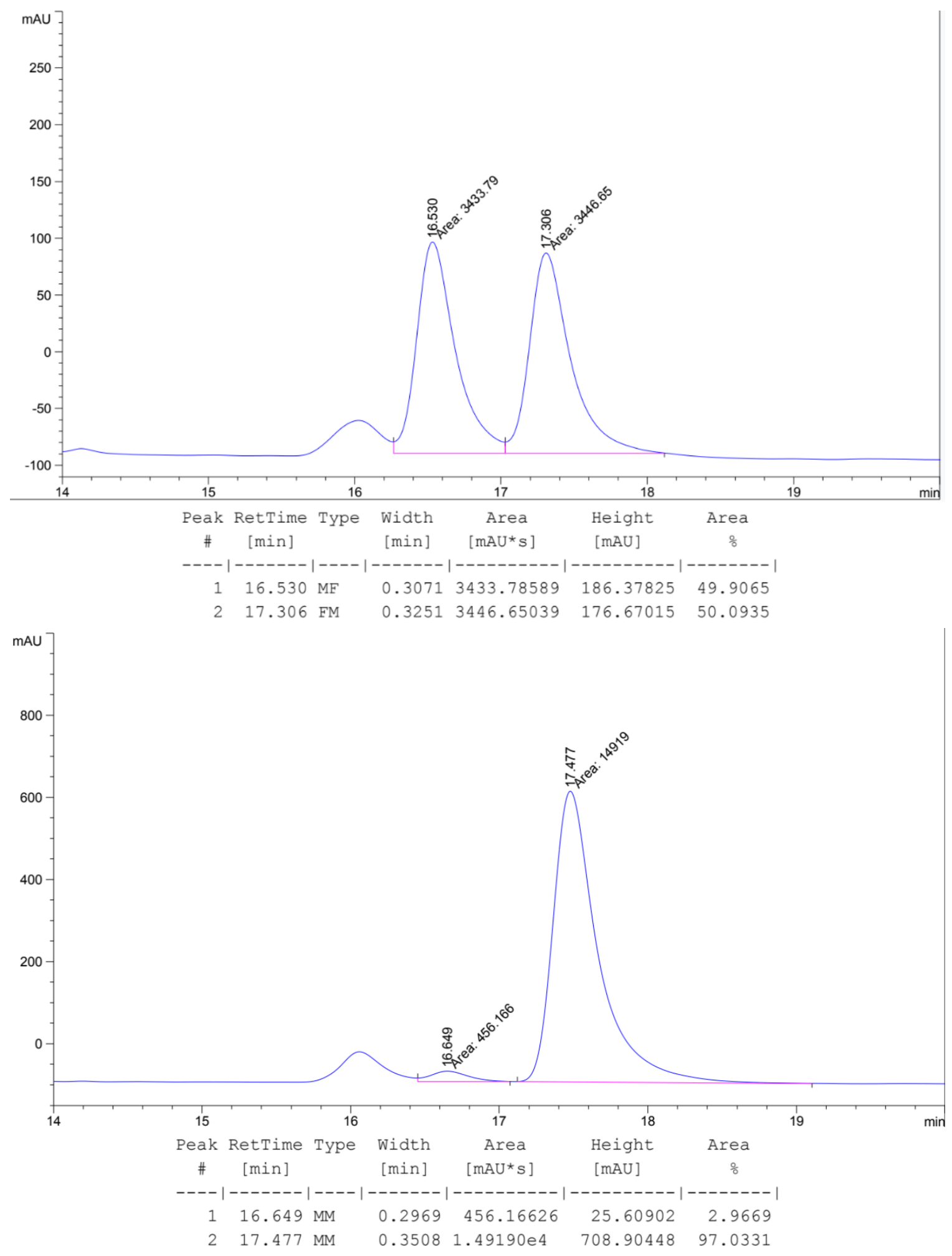


\section{(3S,4S)-3-(benzhydryloxy)-3-methyl-7-((triisopropylsilyl)oxy)hept-1-en-4-ol (4x)}

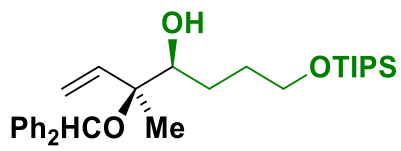

Aldehyde oxidation level: Aldehyde $3 \times$ ( $49.0 \mathrm{mg}, 0.2 \mathrm{mmol}$ ) was subjected to standard reaction conditions $\left(75^{\circ} \mathrm{C}, 24 \mathrm{~h}\right)$. Upon flash column chromatography $\left(\mathrm{SiO}_{2}, 2: 98 \mathrm{EtOAc}\right.$ :hexanes), the title compound $4 \mathbf{x}$ was obtained as a colorless oil in $66 \%$ yield $(63.7 \mathrm{mg}, 0.132 \mathrm{mmol}, 14: 1 \mathrm{dr}, 90 \%$ ee).

$\operatorname{TLC}\left(\mathrm{SiO}_{2}\right) \mathrm{R}_{\mathrm{f}}=0.32$ (5:95 EtOAc:hexanes)

${ }^{1} \mathbf{H}$ NMR $\left(500 \mathrm{MHz}, \mathrm{CDCl}_{3}\right) \delta: 7.35-7.26(\mathrm{~m}, 8 \mathrm{H}), 7.23-7.16(\mathrm{~m}, 2 \mathrm{H}), 5.75(\mathrm{dd}, J=17.6,11.0 \mathrm{~Hz}$, $1 \mathrm{H}), 5.53(\mathrm{~s}, 1 \mathrm{H}), 5.30-5.20(\mathrm{~m}, 2 \mathrm{H}), 3.74(\mathrm{dt}, J=9.8,6.0 \mathrm{~Hz}, 1 \mathrm{H}), 3.68(\mathrm{dt}, J=9.7,6.1 \mathrm{~Hz}, 1 \mathrm{H})$, $3.62(\mathrm{dt}, J=10.6,2.5 \mathrm{~Hz}, 1 \mathrm{H}), 2.81-2.77(\mathrm{~m}, 1 \mathrm{H}), 1.87-1.77(\mathrm{~m}, 1 \mathrm{H}), 1.68-1.55(\mathrm{~m}, 2 \mathrm{H}), 1.30$ (ddt, $J=15.1,10.5,7.6 \mathrm{~Hz}, 1 \mathrm{H}), 1.14(\mathrm{~s}, 3 \mathrm{H}), 1.05(\mathrm{~d}, J=5.1 \mathrm{~Hz}, 21 \mathrm{H})$.

${ }^{13} \mathrm{C}$ NMR $\left(125 \mathrm{MHz}, \mathrm{CDCl}_{3}\right) \delta: 144.9,144.4,140.5,128.5,128.4,127.1,127.1,126.9,126.8,118.1$, $83.3,77.6,76.6,63.4,30.3,27.6,18.2,16.4,12.1$.

HRMS $(\mathrm{Na}+, \mathrm{m} / z)$ for $\mathrm{C}_{30} \mathrm{H}_{46} \mathrm{O}_{3} \mathrm{Si}$ : calcd. $=505.3108$; found $=505.3113$.

FTIR (neat): 2988, 2359, 1453, 1263, 1085, 883, $748 \mathrm{~cm}^{-1}$

HPLC: (Two connected Chiralcel AD-H columns, Hexane:2-PrOH = 99:01, $0.5 \mathrm{~mL} / \mathrm{min}, 230 \mathrm{~nm}$ ) $[\alpha]_{D}^{24}=+14.3^{\circ}\left(\mathrm{c}=0.35, \mathrm{CHCl}_{3}\right)$. 

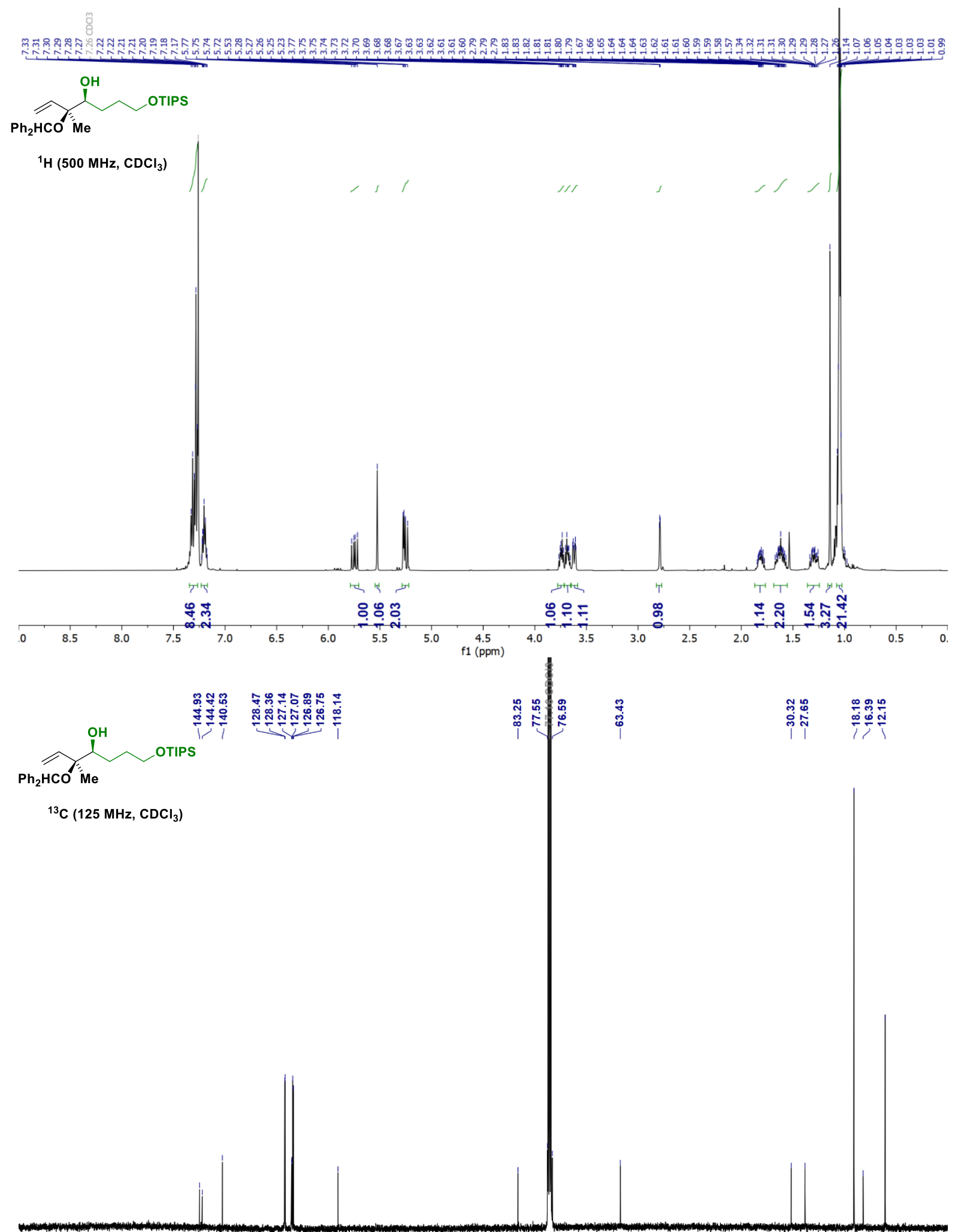

$30 \quad 170 \quad 160 \quad 150 \quad 140 \quad 130 \quad 120 \quad 110,100$ 90
$\mathrm{f} 1(\mathrm{ppm})$ 

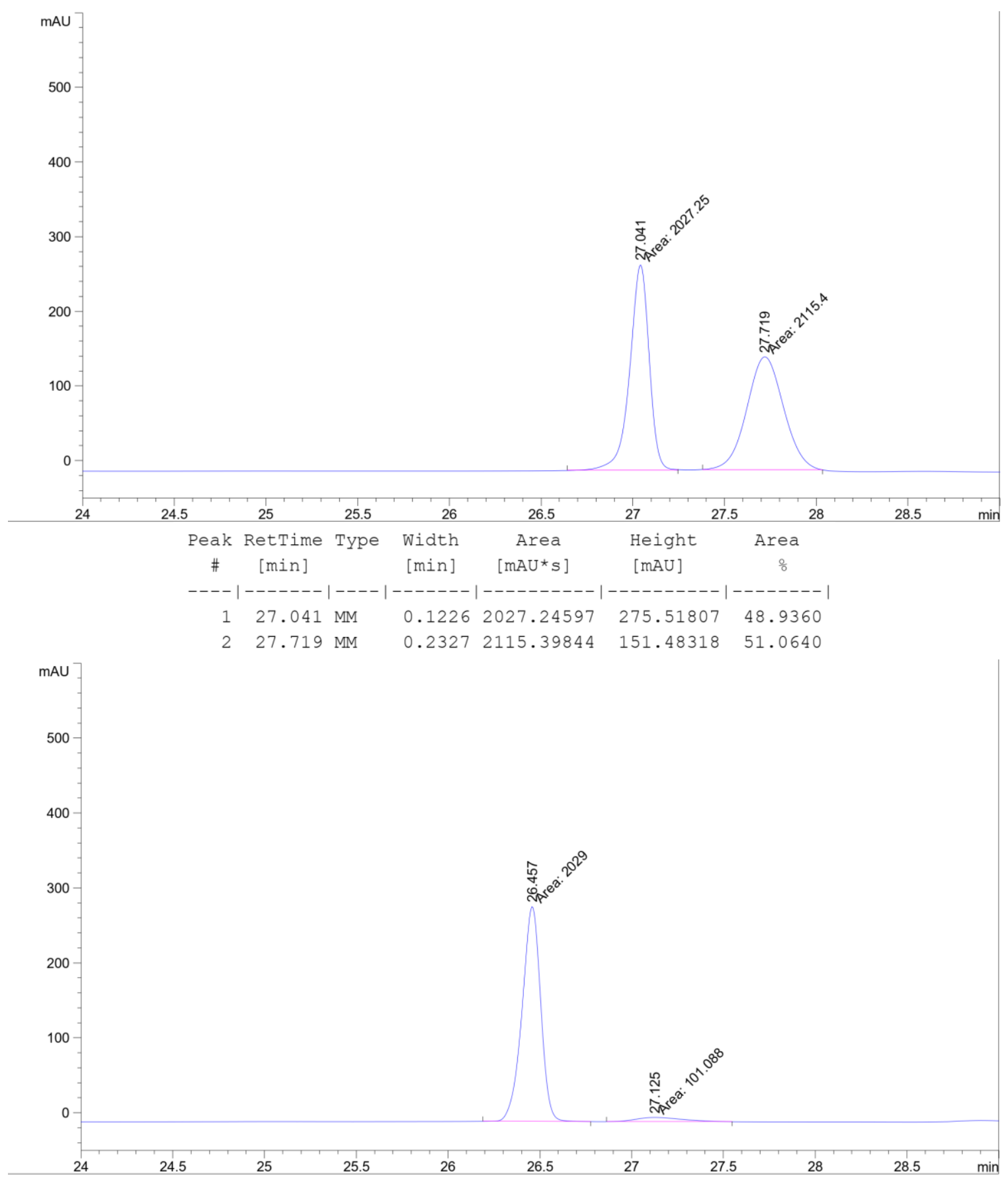

\begin{tabular}{|c|c|c|c|c|c|c|}
\hline $\begin{array}{c}\text { Peak } \\
\quad \#\end{array}$ & $\begin{array}{c}\text { RetTime } \\
\text { [min] }\end{array}$ & Type & $\begin{array}{l}\text { Width } \\
\text { [min] }\end{array}$ & $\begin{array}{c}\text { Area } \\
{\left[\mathrm{mAU}{ }^{\star} \mathrm{s}\right]}\end{array}$ & $\begin{array}{l}\text { Height } \\
{[\mathrm{mAU}]}\end{array}$ & $\begin{array}{c}\text { Area } \\
\frac{o}{8}\end{array}$ \\
\hline & & & & | --------- & ---------- & -- \\
\hline 1 & 26.457 & $\mathrm{MM}$ & 0.1177 & 2029.00012 & 287.22113 & 95.2543 \\
\hline 2 & 27.125 & $\mathrm{MM}$ & 0.2977 & 101.08840 & 5.65910 & 4.7457 \\
\hline
\end{tabular}


(2S,3S)-3-(benzhydryloxy)-1-(4-fluorophenyl)-3-methylpent-4-en-2-ol (4y)

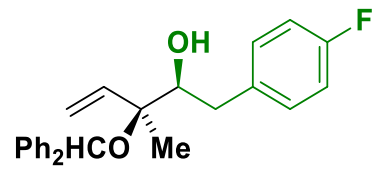

Aldehyde oxidation level: Aldehyde $3 y(27.6 \mathrm{mg}, 0.2 \mathrm{mmol})$ was subjected to standard reaction conditions $\left(75^{\circ} \mathrm{C}, 24 \mathrm{~h}\right)$. Upon flash column chromatography $\left(\mathrm{SiO}_{2}, 5: 95 \mathrm{EtOAc}\right.$ :hexanes), the title compound $4 y$ was obtained as a colorless oil in $62 \%$ yield $(46.2 \mathrm{mg}, 0.124 \mathrm{mmol}, 8: 1 \mathrm{dr}, 96 \%$ ee).

$\operatorname{TLC}\left(\mathrm{SiO}_{2}\right) \mathrm{R}_{\mathrm{f}}=0.35$ (10:90 EtOAc:hexanes)

${ }^{1} \mathbf{H}$ NMR $\left(500 \mathrm{MHz}, \mathrm{CDCl}_{3}\right) \delta: 7.33-7.26(\mathrm{~m}, 4 \mathrm{H}), 7.26-7.21(\mathrm{~m}, 4 \mathrm{H}), 7.17$ (ddd, J = 9.6, 5.3, 2.4 $\mathrm{Hz}, 4 \mathrm{H}), 6.95(\mathrm{td}, J=8.8,2.6 \mathrm{~Hz}, 2 \mathrm{H}), 5.79(\mathrm{dd}, J=17.5,11.0 \mathrm{~Hz}, 1 \mathrm{H}), 5.54(\mathrm{~d}, J=2.2 \mathrm{~Hz}, 1 \mathrm{H}), 5.36$ $-5.28(\mathrm{~m}, 2 \mathrm{H}), 3.79(\mathrm{dt}, J=10.4,2.3 \mathrm{~Hz}, 1 \mathrm{H}), 2.75(\mathrm{~d}, J=14.1 \mathrm{~Hz}, 1 \mathrm{H}), 2.66(\mathrm{dd}, J=2.9,1.5 \mathrm{~Hz}$, $1 \mathrm{H}), 2.47$ (dd, $J=14.1,10.3 \mathrm{~Hz}, 1 \mathrm{H}), 1.21(\mathrm{~s}, 3 \mathrm{H})$.

${ }^{13} \mathrm{C}$ NMR (125 MHz, $\left.\mathrm{CDCl}_{3}\right) \delta: 161.7(\mathrm{~d}, J=243.6 \mathrm{~Hz}), 144.8,144.2,140.2,135.5(\mathrm{~d}, J=3.2 \mathrm{~Hz})$, 130.7 (d, $J=7.7 \mathrm{~Hz}), 128.5,128.4,127.3,127.2,126.8,126.7,118.8,115.2(\mathrm{~d}, J=21.1 \mathrm{~Hz}) .83 .0$, $78.8,76.8,36.9,16.4$.

${ }^{19}$ F NMR $\left(470 \mathrm{MHz}, \mathrm{CDCl}_{3}\right):-117.1--117.7(\mathrm{~m})$

HRMS $(\mathrm{M}+, m / z)$ for $\mathrm{C}_{25} \mathrm{H}_{25} \mathrm{FO}_{2}$ : calcd. $=399.1731 ;$ found $=399.1738$.

FTIR (neat): 3005, 2359, 1508, 1275, 1260, 1082, 1025, 749, $699 \mathrm{~cm}^{-1}$

HPLC: (Two connected Chiralcel AD-H columns, Hexane:2-PrOH =98:02, $0.5 \mathrm{~mL} / \mathrm{min}, 210 \mathrm{~nm}$ ) $[\alpha]_{D}^{24}=+20.0^{\circ}\left(\mathrm{c}=0.25, \mathrm{CHCl}_{3}\right)$. 

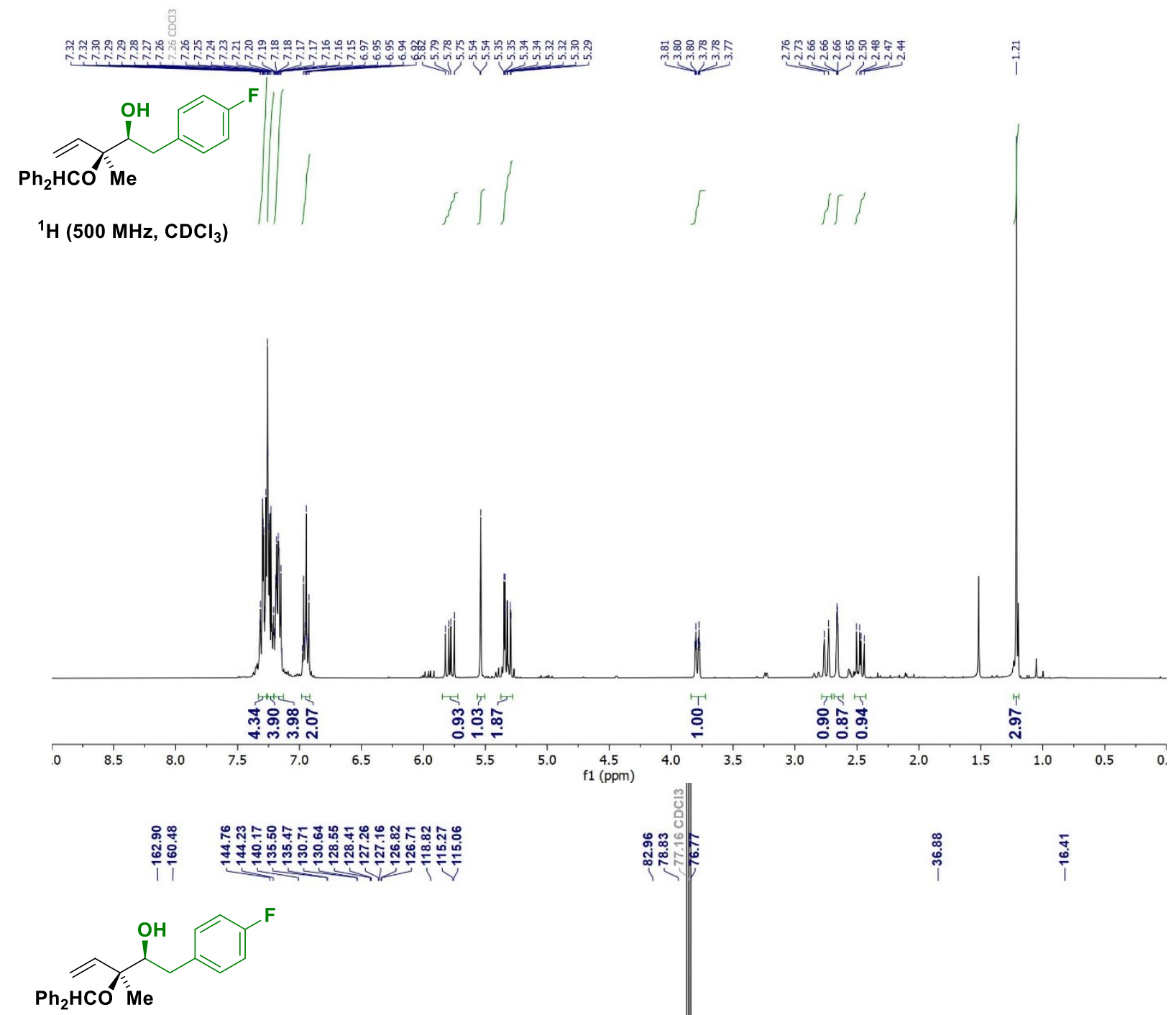

${ }^{13} \mathrm{C}\left(125 \mathrm{MHz}, \mathrm{CDCl}_{3}\right)$

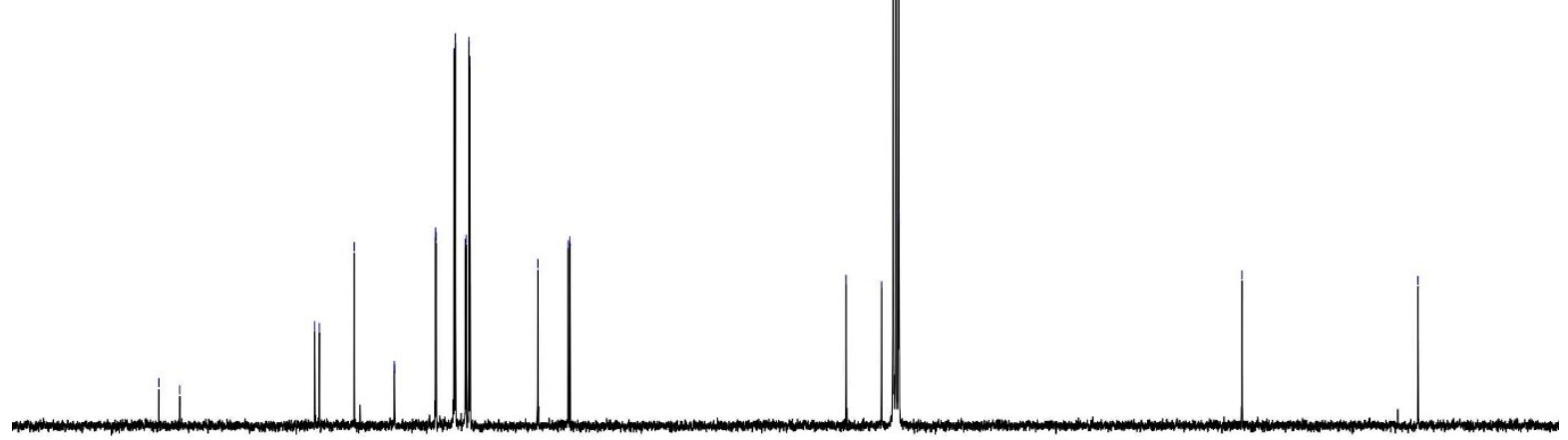

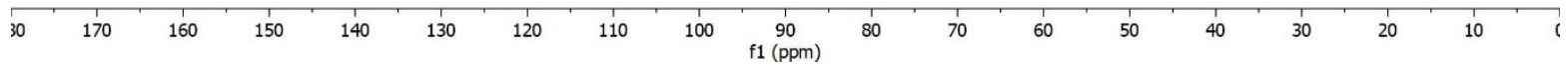




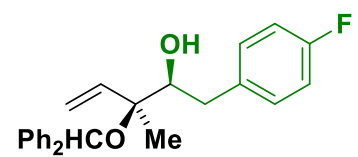

${ }^{19} \mathrm{~F}\left(470 \mathrm{MHz}, \mathrm{CDCl}_{3}\right)$

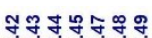

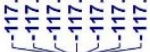

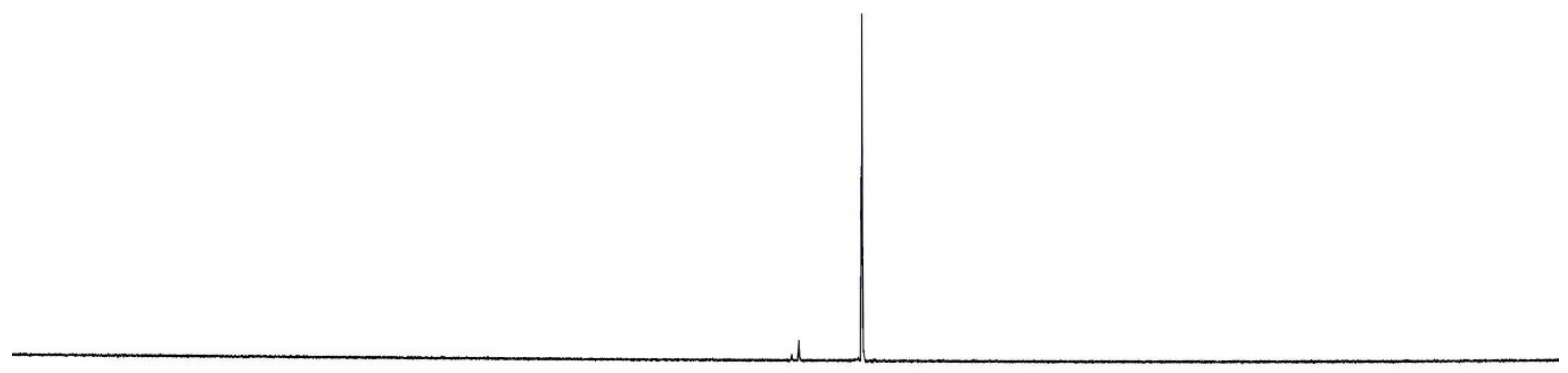

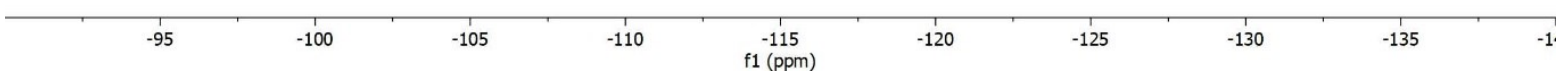




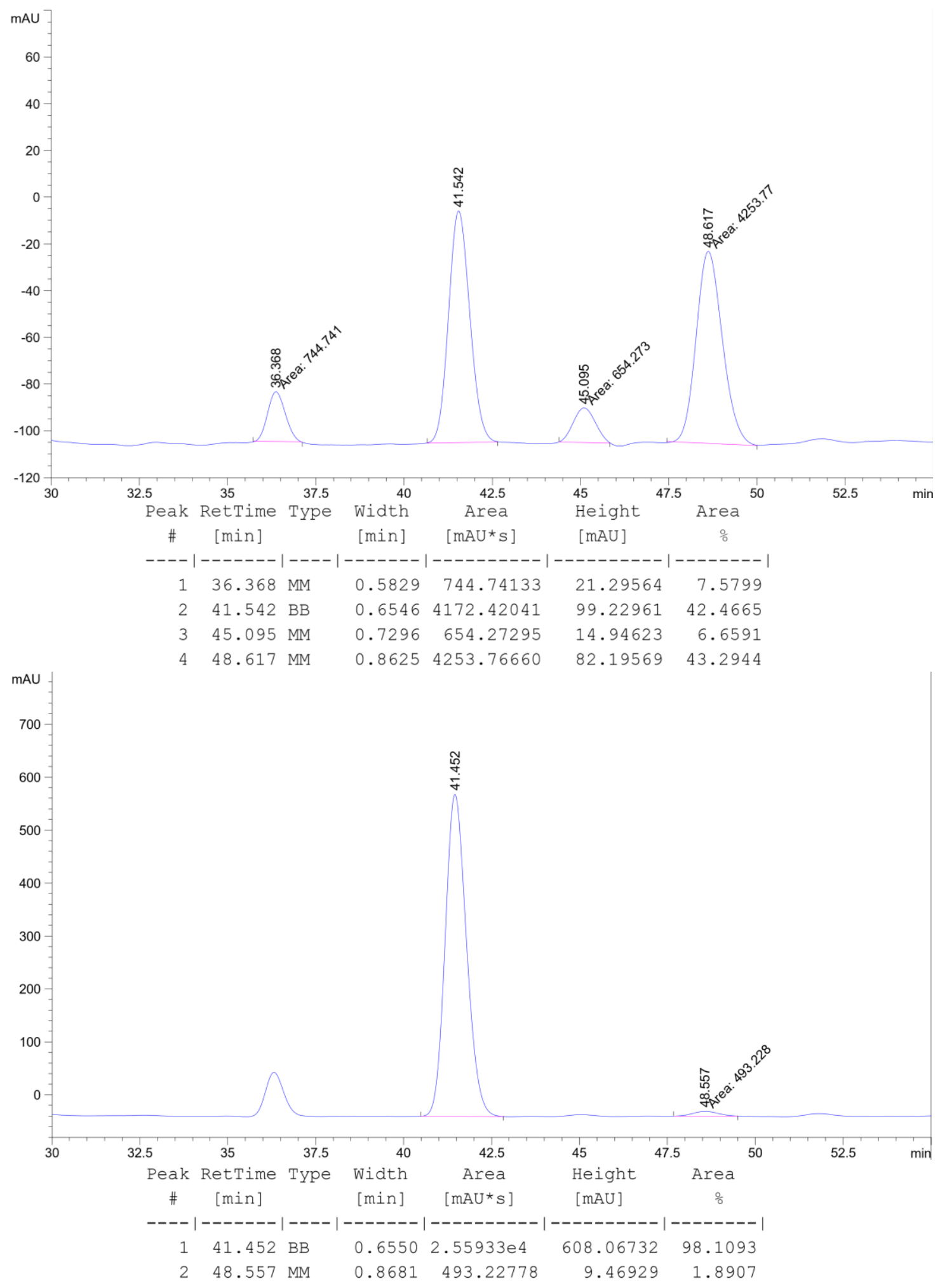


tert-butyl (4-((3S,4S)-4-(benzhydryloxy)-3-hydroxy-4-methylhex-5-en-1-yl)-2-

fluorophenyl)carbamate (4z)

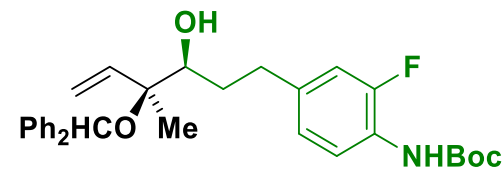

Aldehyde oxidation level: Aldehyde $3 z(27.6 \mathrm{mg}, 0.2 \mathrm{mmol})$ was subjected to standard reaction conditions $\left(75^{\circ} \mathrm{C}, 24 \mathrm{~h}\right)$. Upon flash column chromatography $\left(\mathrm{SiO}_{2}, 8: 92 \mathrm{EtOAc}\right.$ :hexanes), the title compound $4 z$ was obtained as a yellow oil in $65 \%$ yield $(65.7 \mathrm{mg}, 0.13 \mathrm{mmol}, 9: 1 \mathrm{dr}, 92 \%$ ee).

$\operatorname{TLC}\left(\mathrm{SiO}_{2}\right) \mathrm{R}_{\mathrm{f}}=0.35$ (15:85 EtOAc:hexanes)

${ }^{1} \mathbf{H}$ NMR $\left(500 \mathrm{MHz}, \mathrm{CDCl}_{3}\right) \delta: 7.92(\mathrm{~s}, 1 \mathrm{H}), 7.31-7.26(\mathrm{~m}, 6 \mathrm{H}), 7.26(\mathrm{~d}, J=3.0 \mathrm{~Hz}, 2 \mathrm{H}), 7.20$ (ddt, J $=9.6,4.6,2.2 \mathrm{~Hz}, 2 \mathrm{H}), 6.94-6.85(\mathrm{~m}, 2 \mathrm{H}), 6.60(\mathrm{~s}, 1 \mathrm{H}), 5.68(\mathrm{dd}, J=17.6,10.9 \mathrm{~Hz}, 1 \mathrm{H}), 5.50(\mathrm{~s}$, $1 \mathrm{H}), 5.30-5.17(\mathrm{~m}, 2 \mathrm{H}), 3.57(\mathrm{dt}, J=10.5,2.5 \mathrm{~Hz}, 1 \mathrm{H}), 2.85(\mathrm{td}, J=9.6,4.8 \mathrm{~Hz}, 1 \mathrm{H}), 2.70(\mathrm{dd}, J=$ $3.2,1.5 \mathrm{~Hz}, 1 \mathrm{H}), 2.58(\mathrm{ddd}, J=13.9,9.2,7.5 \mathrm{~Hz}, 1 \mathrm{H}), 1.74-1.61(\mathrm{~m}, 1 \mathrm{H}), 1.60-1.52(\mathrm{~m}, 1 \mathrm{H}), 1.52$ $(\mathrm{s}, 9 \mathrm{H}), 1.12(\mathrm{~s}, 3 \mathrm{H})$.

${ }^{13} \mathrm{C}$ NMR $\left(125 \mathrm{MHz}, \mathrm{CDCl}_{3}\right) \delta: 152.7,152.2(\mathrm{~d}, J=241.9 \mathrm{~Hz}), 144.7,144.2,140.3,137.8(\mathrm{~d}, J=6.8$ $\mathrm{Hz}), 128.5,128.4,127.2,127.2,126.9,126.7,126.7,124.5$ (q, J = 4.5, 3.6 Hz), 120.3, 118.5, 114.9 $(d, J=18.8 \mathrm{~Hz}), 83.1,81.0,76.7,76.4,32.8,32.2,28.4,16.1$.

${ }^{19} \mathrm{~F} \mathrm{NMR}\left(470 \mathrm{MHz}, \mathrm{CDCl}_{3}\right) \delta:-132.88$.

HRMS $(\mathrm{M}+, m / z)$ for $\mathrm{C}_{25} \mathrm{H}_{25} \mathrm{FO}_{2}$ : calcd. = 399.1731; found $=399.1738$.

FTIR (neat): 3005, 2988, 2360, 1729, 1527, 1261, 1158, 1023, $749 \mathrm{~cm}^{-1}$

HPLC: (Two connected Chiralcel columns AD-H \& ODH, Hexane:2-PrOH = 98:02, $0.5 \mathrm{~mL} / \mathrm{min}$, $210 \mathrm{~nm})$

$[\alpha]_{D}^{24}=+57.9^{\circ}\left(\mathrm{c}=0.38, \mathrm{CHCl}_{3}\right)$. 


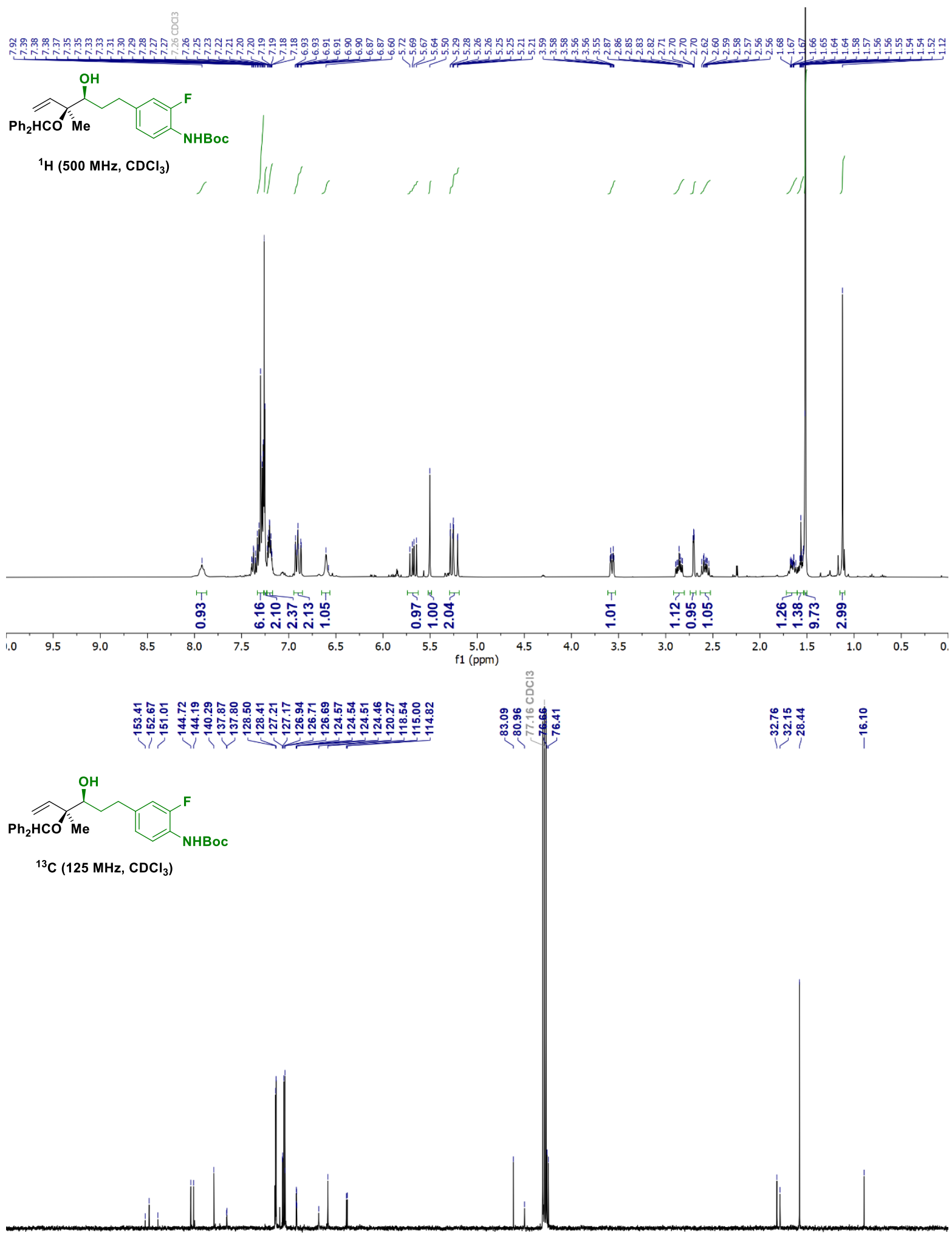

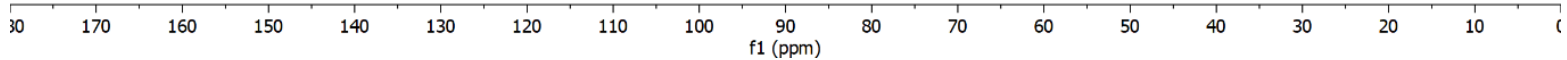




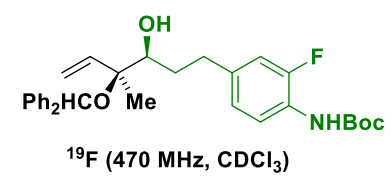

${ }^{19} \mathrm{~F}\left(470 \mathrm{MHz}, \mathrm{CDCl}_{3}\right)$

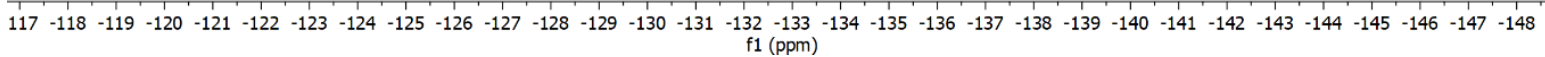




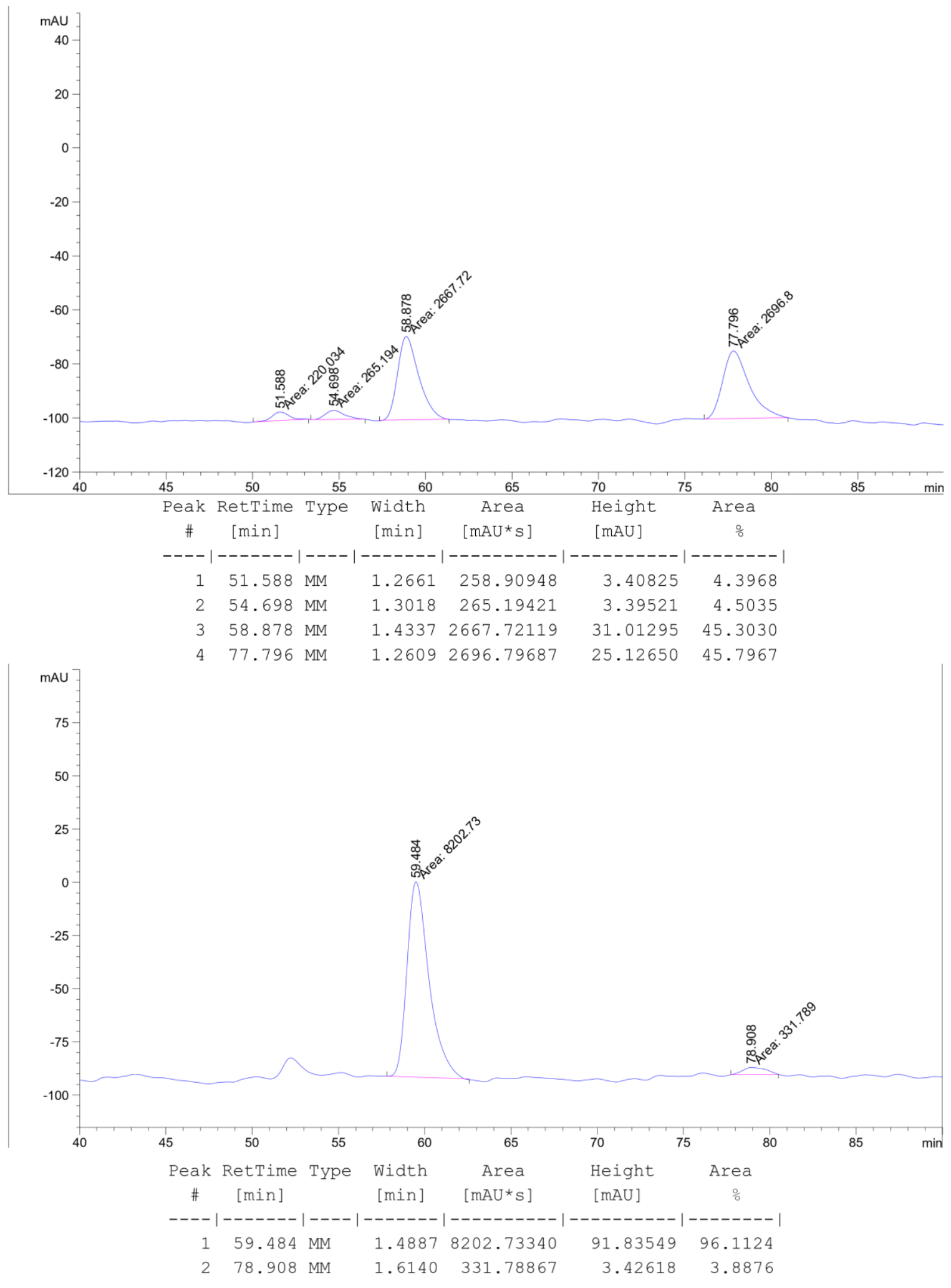


methyl 4-((3-((3S,4S)-4-(benzhydryloxy)-3-hydroxy-4-methylhex-5-en-1-yl)-1H-indol-1yl)sulfonyl)benzoate (4aa)

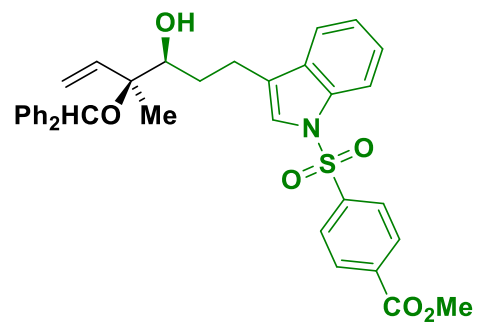

Aldehyde oxidation level: Aldehyde $3 a a(27.6 \mathrm{mg}, 0.2 \mathrm{mmol})$ was subjected to standard reaction conditions $\left(75^{\circ} \mathrm{C}, 24 \mathrm{~h}\right.$ ). Upon flash column chromatography $\left(\mathrm{SiO}_{2}, 20: 80\right.$ EtOAc:hexanes), the title compound 4 aa was obtained as a yellow solid in $59 \%$ yield $(71.9 \mathrm{mg}, 0.118 \mathrm{mmol}, 11: 1 \mathrm{dr}$, $90 \%$ ee).

$\operatorname{TLC}\left(\mathrm{SiO}_{2}\right) \mathrm{R}_{\mathrm{f}}=0.25$ (20:80 EtOAc:hexanes)

${ }^{1} \mathbf{H}$ NMR $\left(500 \mathrm{MHz}, \mathrm{CDCl}_{3}\right) \delta: 8.07-8.01(\mathrm{~m}, 2 \mathrm{H}), 7.96(\mathrm{~d}, J=8.2 \mathrm{~Hz}, 1 \mathrm{H}), 7.90-7.85(\mathrm{~m}, 2 \mathrm{H}), 7.48$ $(\mathrm{d}, J=7.8 \mathrm{~Hz}, 1 \mathrm{H}), 7.32-7.27(\mathrm{~m}, 10 \mathrm{H}), 7.25-7.18(\mathrm{~m}, 3 \mathrm{H}), 5.70(\mathrm{dd}, J=17.6,11.0 \mathrm{~Hz}, 1 \mathrm{H}), 5.52$ $(\mathrm{s}, 1 \mathrm{H}), 5.32-5.20(\mathrm{~m}, 2 \mathrm{H}), 3.89(\mathrm{~s}, 3 \mathrm{H}), 3.65(\mathrm{dt}, J=10.8,2.3 \mathrm{~Hz}, 1 \mathrm{H}), 2.96(\mathrm{ddd}, J=14.4,9.6,4.6$ $\mathrm{Hz}, 1 \mathrm{H}), 2.73(\mathrm{dd}, J=3.4,1.6 \mathrm{~Hz}, 1 \mathrm{H}), 2.72-2.65(\mathrm{~m}, 1 \mathrm{H}), 1.79(\mathrm{dt}, J=16.5,8.4 \mathrm{~Hz}, 1 \mathrm{H}), 1.63(\mathrm{dtd}$, $J=14.1,9.8,4.7 \mathrm{~Hz}, 1 \mathrm{H}), 1.15(\mathrm{~s}, 3 \mathrm{H})$.

${ }^{13} \mathrm{C}$ NMR $\left(125 \mathrm{MHz}_{\mathrm{CDCl}}\right) \delta$ : 165.4, 144.7, 144.2, 141.9, 140.2, 135.5, 134.8, 131.4, 130.5, 128.5, $128.4,127.3,127.2,126.9,126.8,126.7,125.1,124.3,123.6,122.6,119.9,118.7,113.8,83.1$, $77.4,76.9,52.8,30.5,22.2,16.2$.

HRMS $(\mathrm{Na}+, \mathrm{m} / z)$ for $\mathrm{C}_{36} \mathrm{H}_{35} \mathrm{NO}_{6} \mathrm{~S}$ : calcd. $=632.2077$; found $=632.2076$.

FTIR (neat): 3005, 2360, 2341, 1730, 1448, 1374, 1262, 1180, $1018 \mathrm{~cm}^{-1}$

HPLC: (Two connected Chiralcel AD-H columns, Hexane:2-PrOH = 98:02, $0.5 \mathrm{~mL} / \mathrm{min}, 210 \mathrm{~nm}$ ) $[\alpha]_{D}^{24}=+26.0^{\circ}\left(\mathrm{c}=0.34, \mathrm{CHCl}_{3}\right)$. 

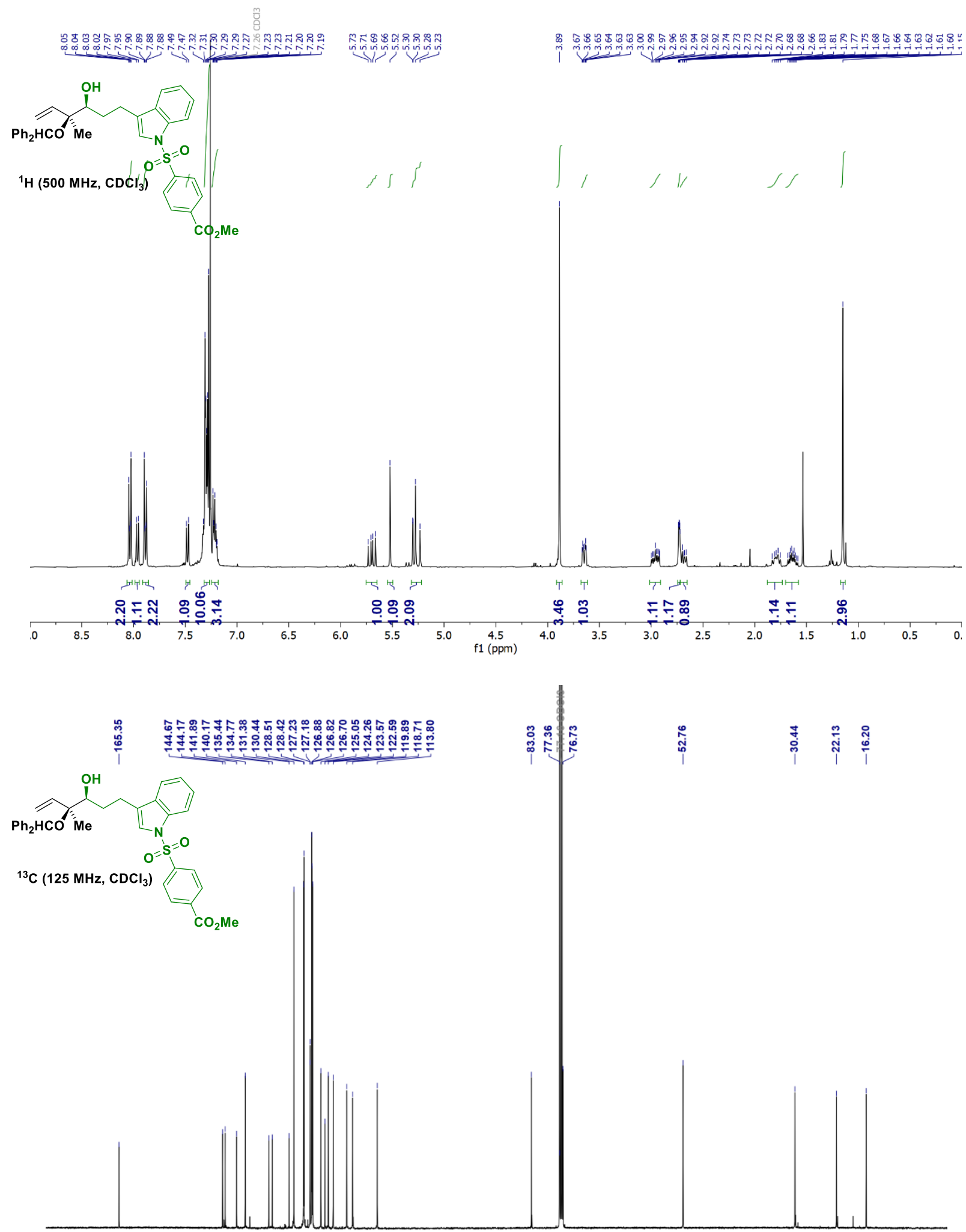

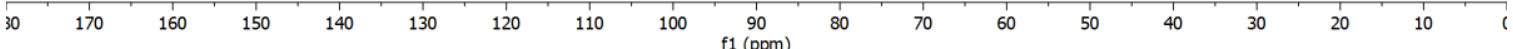




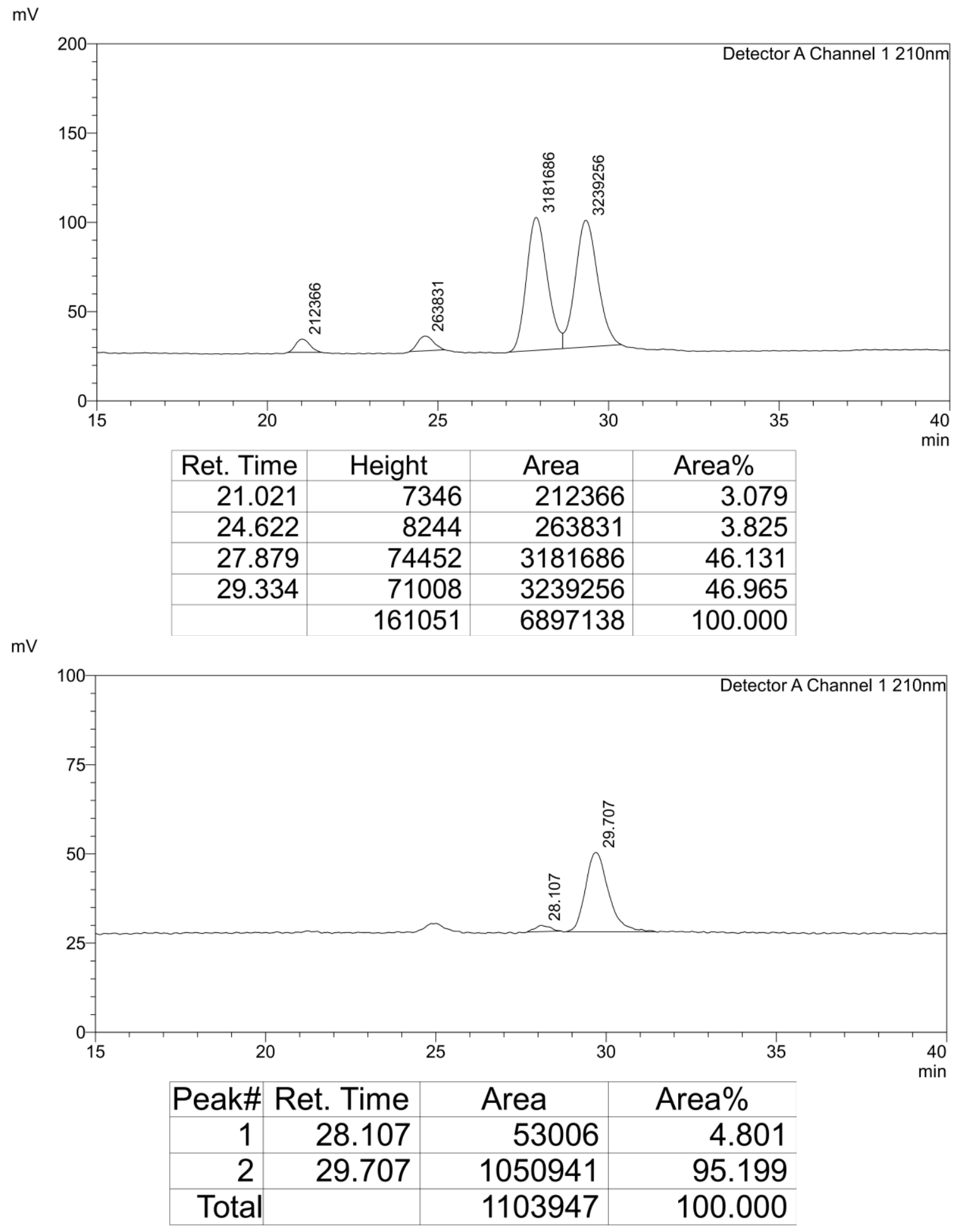


(3S,4S)-4-(benzhydryloxy)-1-(2,2-difluorobenzo[d][1,3]dioxol-5-yl)-4-methylhex-5-en-3-ol (4bb)

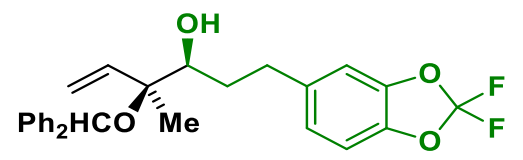

Aldehyde oxidation level: Aldehyde $3 \mathrm{bb}(42.8 \mathrm{mg}, 0.2 \mathrm{mmol})$ was subjected to standard reaction conditions $\left(75^{\circ} \mathrm{C}, 24 \mathrm{~h}\right)$. Upon flash column chromatography $\left(\mathrm{SiO}_{2}, 2: 98\right.$ EtOAc:hexanes $)$, the title compound $\mathbf{4 b b}$ was obtained as a colorless oil in $67 \%$ yield $(60.6 \mathrm{mg}, 0.134 \mathrm{mmol}, 10: 1 \mathrm{dr}, 92 \%$ ee).

$\operatorname{TLC}\left(\mathrm{SiO}_{2}\right) \mathrm{R}_{\mathrm{f}}=0.2$ (8:92 EtOAc:hexanes)

${ }^{1} \mathrm{H}$ NMR $\left(400 \mathrm{MHz}, \mathrm{CDCl}_{3}\right) 7.33-7.26(\mathrm{~m}, 8 \mathrm{H}), 7.21(\mathrm{dtd}, J=7.0,5.2,2.9 \mathrm{~Hz}, 3 \mathrm{H}), 6.98-6.81(\mathrm{~m}$, $3 \mathrm{H}), 5.69(\mathrm{dd}, J=17.6,10.9 \mathrm{~Hz}, 1 \mathrm{H}), 5.52(\mathrm{~s}, 1 \mathrm{H}), 5.35-5.20(\mathrm{~m}, 2 \mathrm{H}), 3.58(\mathrm{dt}, J=10.5,2.3 \mathrm{~Hz}$, $1 \mathrm{H}), 2.91$ (ddd, $J=14.2,9.6,4.9 \mathrm{~Hz}, 1 \mathrm{H}), 2.74(\mathrm{~d}, J=2.5 \mathrm{~Hz}, 1 \mathrm{H}), 2.64$ (ddd, $J=13.9,9.2,7.4 \mathrm{~Hz}$, $1 \mathrm{H}), 1.65(\mathrm{~s}, 1 \mathrm{H}), 1.56(\mathrm{~s}, 1 \mathrm{H}), 1.14(\mathrm{~s}, 3 \mathrm{H})$.

${ }^{13} \mathrm{C}$ NMR $\left(100 \mathrm{MHz}, \mathrm{CDCl}_{3}\right) \delta: 144.5,144.0,143.8,141.9,140.1,138.4,131.6$ (t, $\left.\mathrm{J}=260.8 \mathrm{~Hz}\right)$, 128.4, 128.3, 127.1, 127.1, 126.8, 126.6, 123.3, 118.5, 109.7, 109.1, 82.9, 76.6, 76.2, 32.9, 32.6, 15.9.

${ }^{19}$ F NMR $\left(390 \mathrm{MHz}, \mathrm{CDCl}_{3}\right) \delta:-50.0$.

HRMS $(\mathrm{Na}+, m / z)$ for $\mathrm{C}_{27} \mathrm{H}_{26} \mathrm{~F} 2 \mathrm{O}_{4}$ : calcd. $=475.1691 ;$ found $=475.1693$.

FTIR (neat): 3556, 2926, 2361, 1451, 1237, 1148, 1083, $932 \mathrm{~cm}^{-1}$.

HPLC: (Two connected Chiralcel columns AD-H and OD-H, Hexane:2-PrOH = 98:02, $1.0 \mathrm{~mL} / \mathrm{min}$, $210 \mathrm{~nm})$

$[\alpha]_{D}^{24}=+34.0^{\circ}\left(\mathrm{c}=0.3, \mathrm{CHCl}_{3}\right)$. 


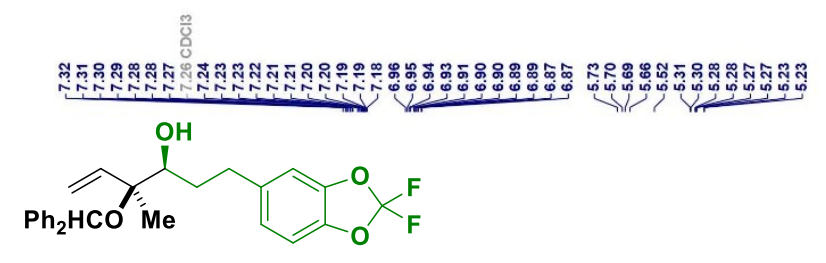

${ }^{1} \mathrm{H}\left(400 \mathrm{MHz}, \mathrm{CDCl}_{3}\right)$

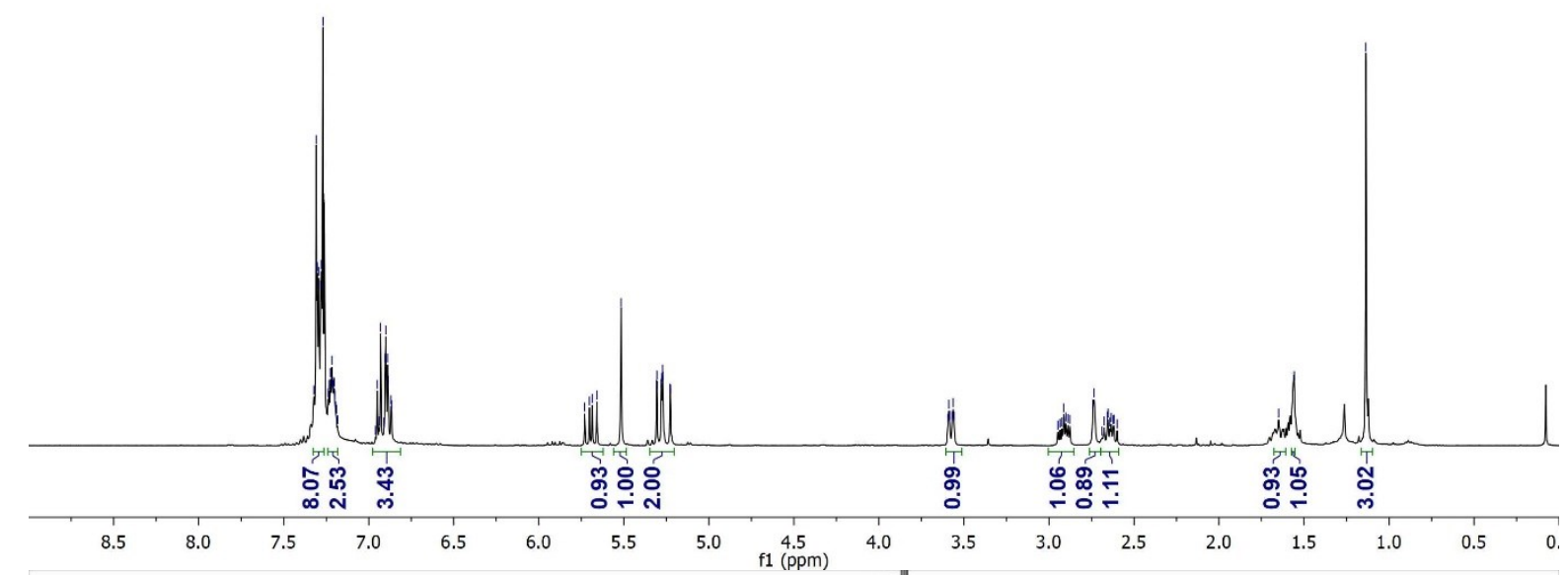

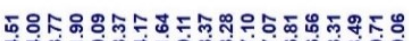

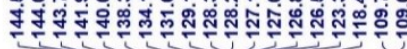



${ }^{13} \mathrm{C}\left(100 \mathrm{MHz}, \mathrm{CDCl}_{3}\right)$

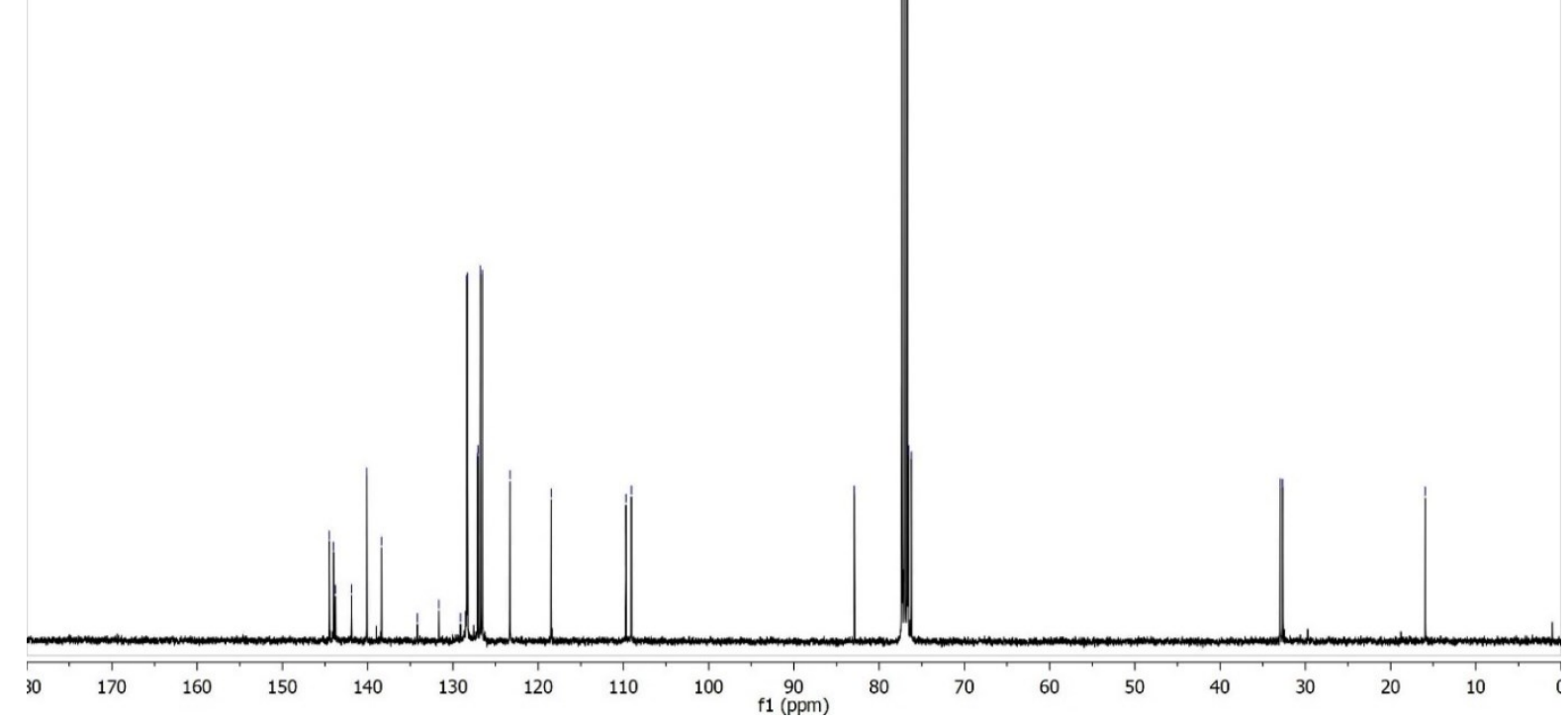




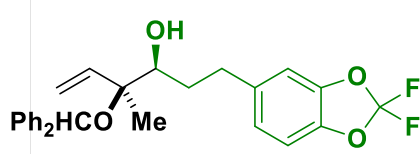

ఫั.

${ }^{19} \mathrm{~F}\left(390 \mathrm{MHz}, \mathrm{CDCl}_{3}\right)$

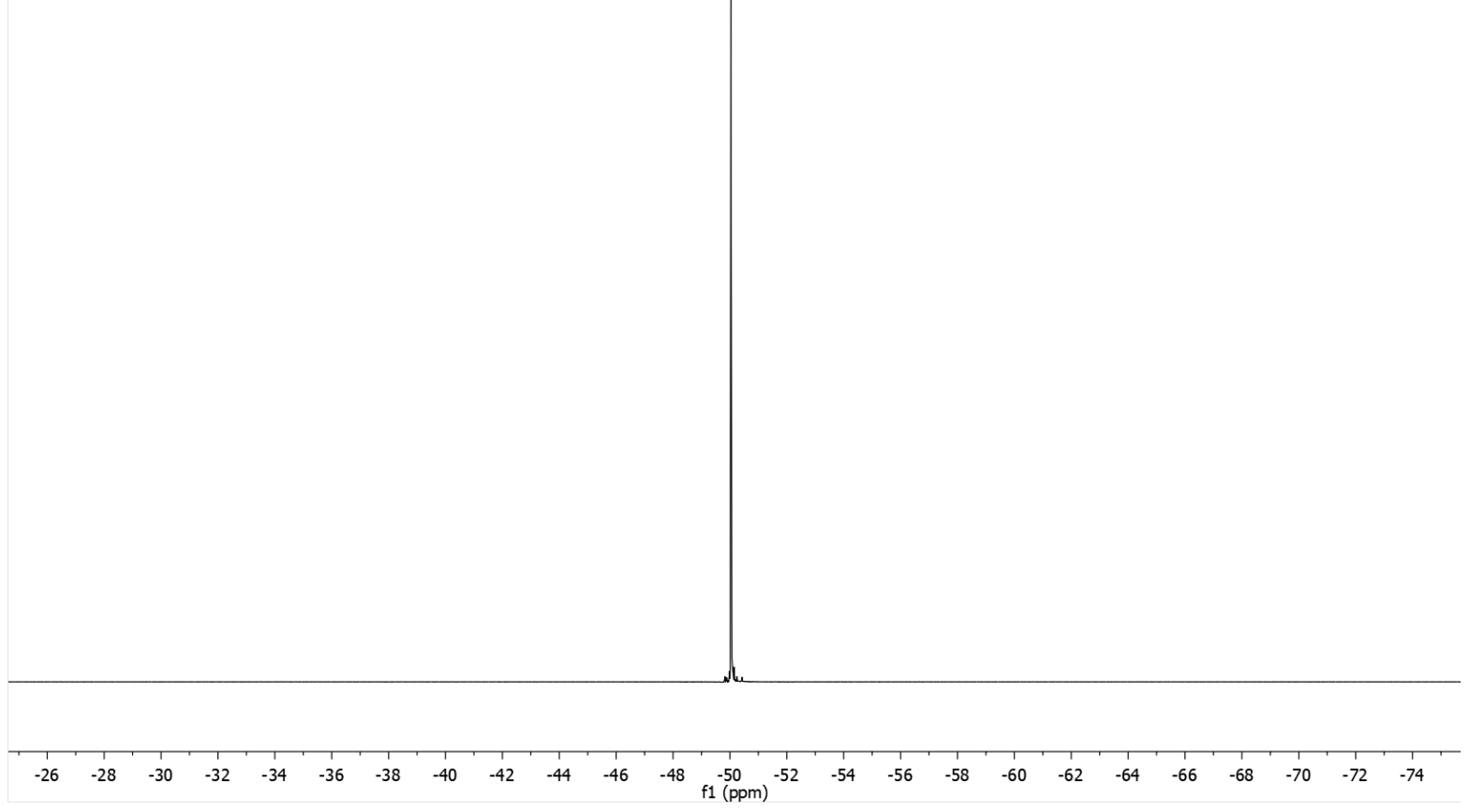



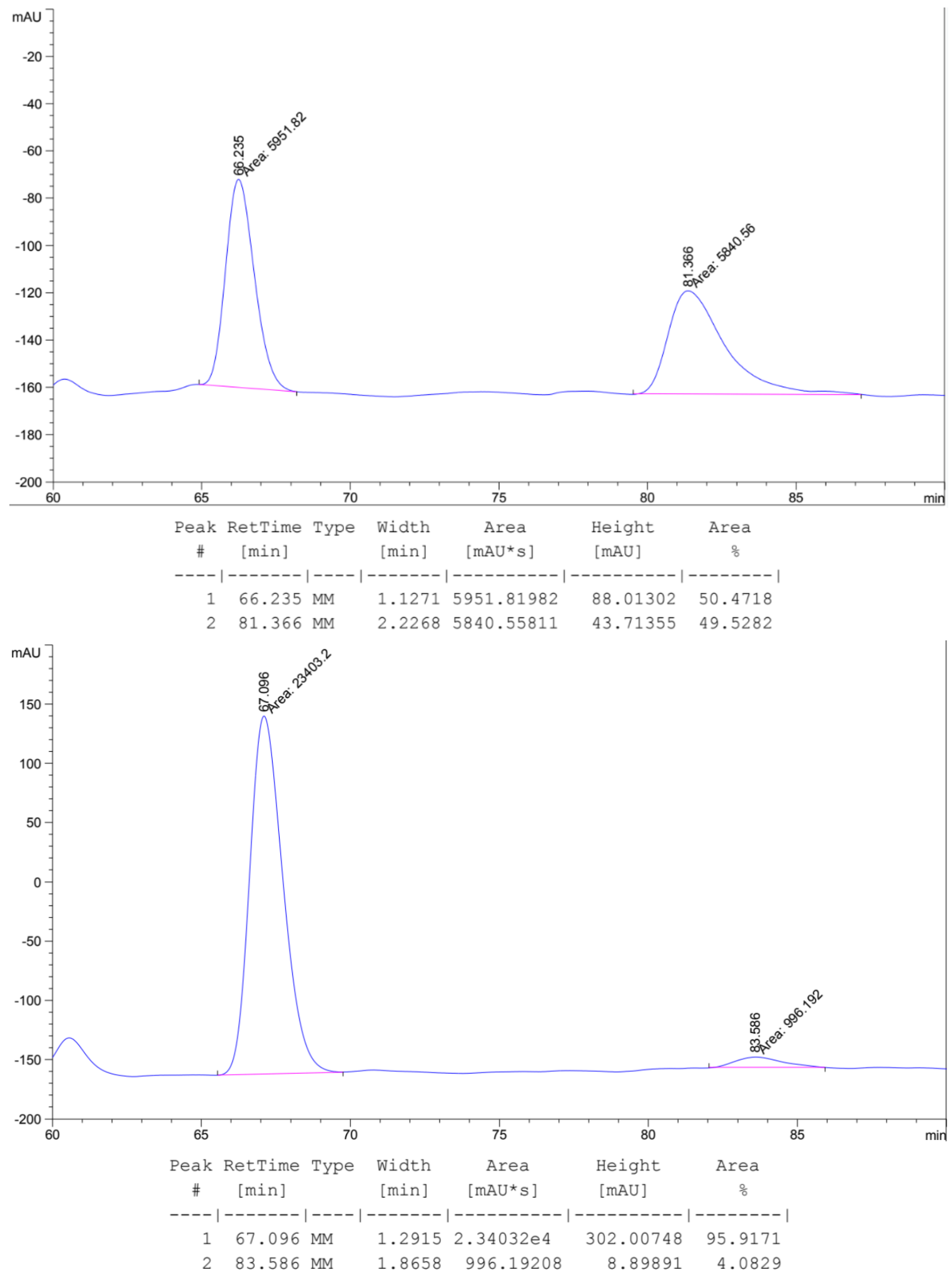
(3S,4S)-4-(benzhydryloxy)-4-methyl-1-(5-methylfuran-2-yl)hex-5-en-3-ol (4cc)

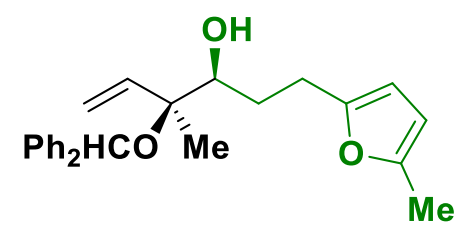

Aldehyde oxidation level: Aldehyde $3 \mathrm{cc}(27.6 \mathrm{mg}, 0.2 \mathrm{mmol})$ was subjected to standard reaction conditions $\left(75^{\circ} \mathrm{C}, 24 \mathrm{~h}\right)$. Upon flash column chromatography $\left(\mathrm{SiO}_{2}, 5: 95 \mathrm{EtOAc}\right.$ :hexanes), the title compound $4 \mathrm{cc}$ was obtained as a yellow oil in $59 \%$ yield $(44.5 \mathrm{mg}, 0.118 \mathrm{mmol}, 12: 1 \mathrm{dr}, 94 \%$ ee).

$\operatorname{TLC}\left(\mathrm{SiO}_{2}\right) \mathrm{R}_{\mathrm{f}}=0.5$ (10:90 EtOAc:hexanes)

${ }^{1} \mathbf{H}$ NMR $\left(500 \mathrm{MHz}, \mathrm{CDCl}_{3}\right) \delta: 7.34-7.21(\mathrm{~m}, 8 \mathrm{H}), 7.21-7.15(\mathrm{~m}, 2 \mathrm{H}), 5.83(\mathrm{~d}, J=3.1 \mathrm{~Hz}, 1 \mathrm{H}), 5.81$ (dd, $J=3.0,1.2 \mathrm{~Hz}, 1 \mathrm{H}), 5.71(\mathrm{dd}, J=17.6,11.0 \mathrm{~Hz}, 1 \mathrm{H}), 5.50(\mathrm{~s}, 1 \mathrm{H}), 5.27(\mathrm{dd}, J=11.1,1.2 \mathrm{~Hz}, 1 \mathrm{H})$, 5.24 (dd, $J=17.6,1.3 \mathrm{~Hz}, 1 \mathrm{H}$ ), 3.61 (ddd, $J=10.6,3.3,1.9 \mathrm{~Hz}, 1 \mathrm{H}$ ), 2.85 (ddd, $J=14.8,9.5,4.8 \mathrm{~Hz}$, $1 \mathrm{H}), 2.67(\mathrm{dd}, J=3.3,1.6 \mathrm{~Hz}, 1 \mathrm{H}), 2.61(\mathrm{ddd}, J=15.7,9.1,7.2 \mathrm{~Hz}, 1 \mathrm{H}), 2.22(\mathrm{~s}, 3 \mathrm{H}), 1.80-1.71(\mathrm{~m}$, $1 \mathrm{H}), 1.60-1.50(\mathrm{~m}, 1 \mathrm{H}), 1.12(\mathrm{~s}, 3 \mathrm{H})$.

${ }^{13} \mathrm{C}$ NMR $\left(125 \mathrm{MHz}, \mathrm{CDCl}_{3}\right)$ $\delta: 154.3,150.4,144.8,144.3,140.3,128.5,128.4,127.2,127.1,126.9$, $126.7,118.5,105.9,105.6,83.1,76.8,76.6,29.9,25.4,16.2,13.7$.

HRMS $(\mathrm{Na}+, m / z)$ for $\mathrm{C}_{25} \mathrm{H}_{28} \mathrm{O}_{3}$ : calcd. = 399.1931; found $=399.1918$.

FTIR (neat): 2937, 1712, 1451, 1355, 1083, 1022, 921, 742, $700 \mathrm{~cm}^{-1}$

HPLC: (Two connected Chiralcel columns AD-H and OD-H, Hexane:2-PrOH = 98:02, $0.5 \mathrm{~mL} / \mathrm{min}$, $210 \mathrm{~nm})$

$[\alpha]_{D}^{24}=+2.0^{\circ}\left(\mathrm{c}=1.0, \mathrm{CHCl}_{3}\right)$ 


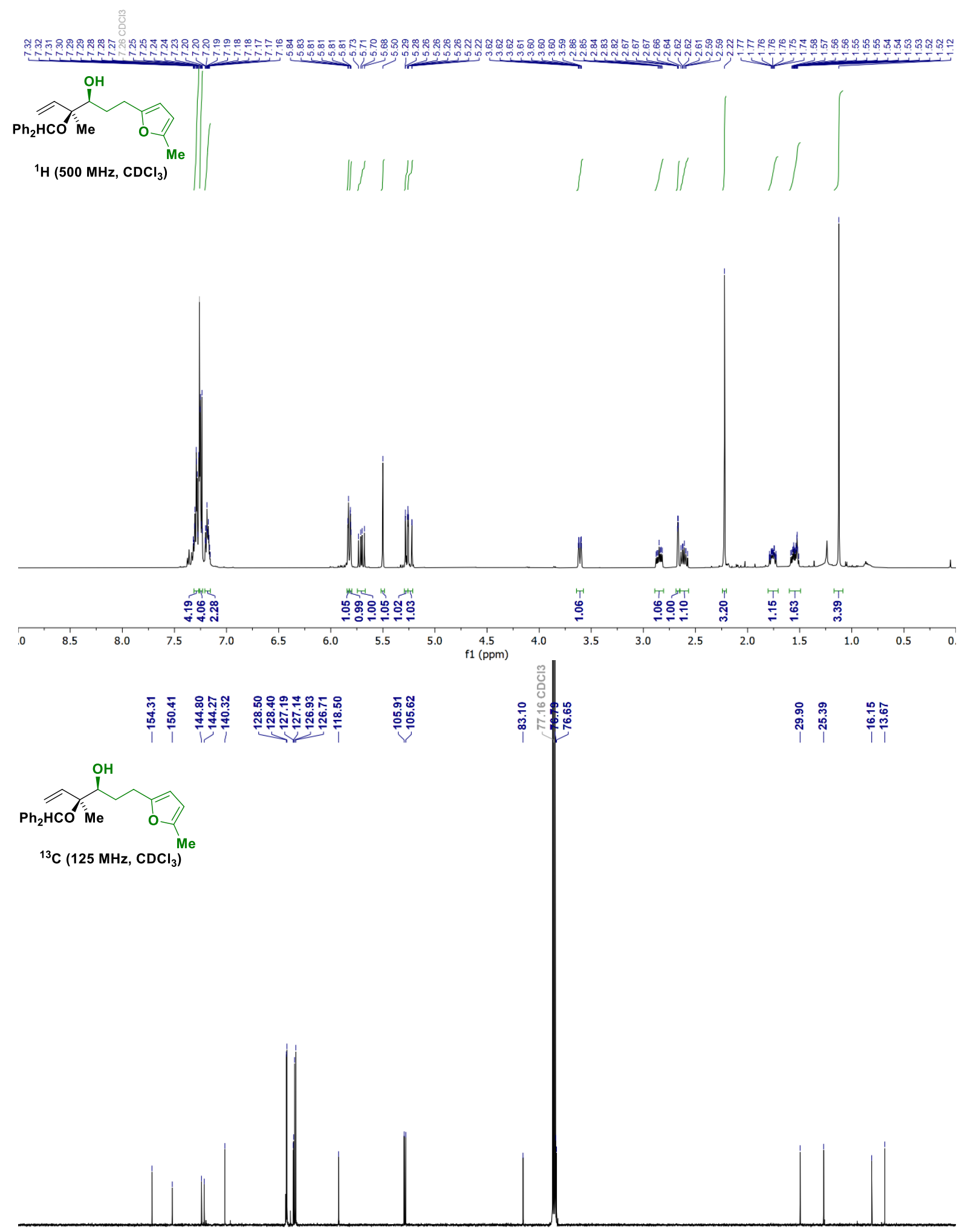

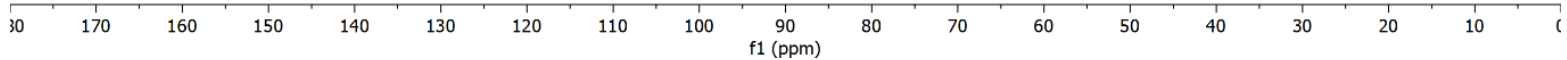



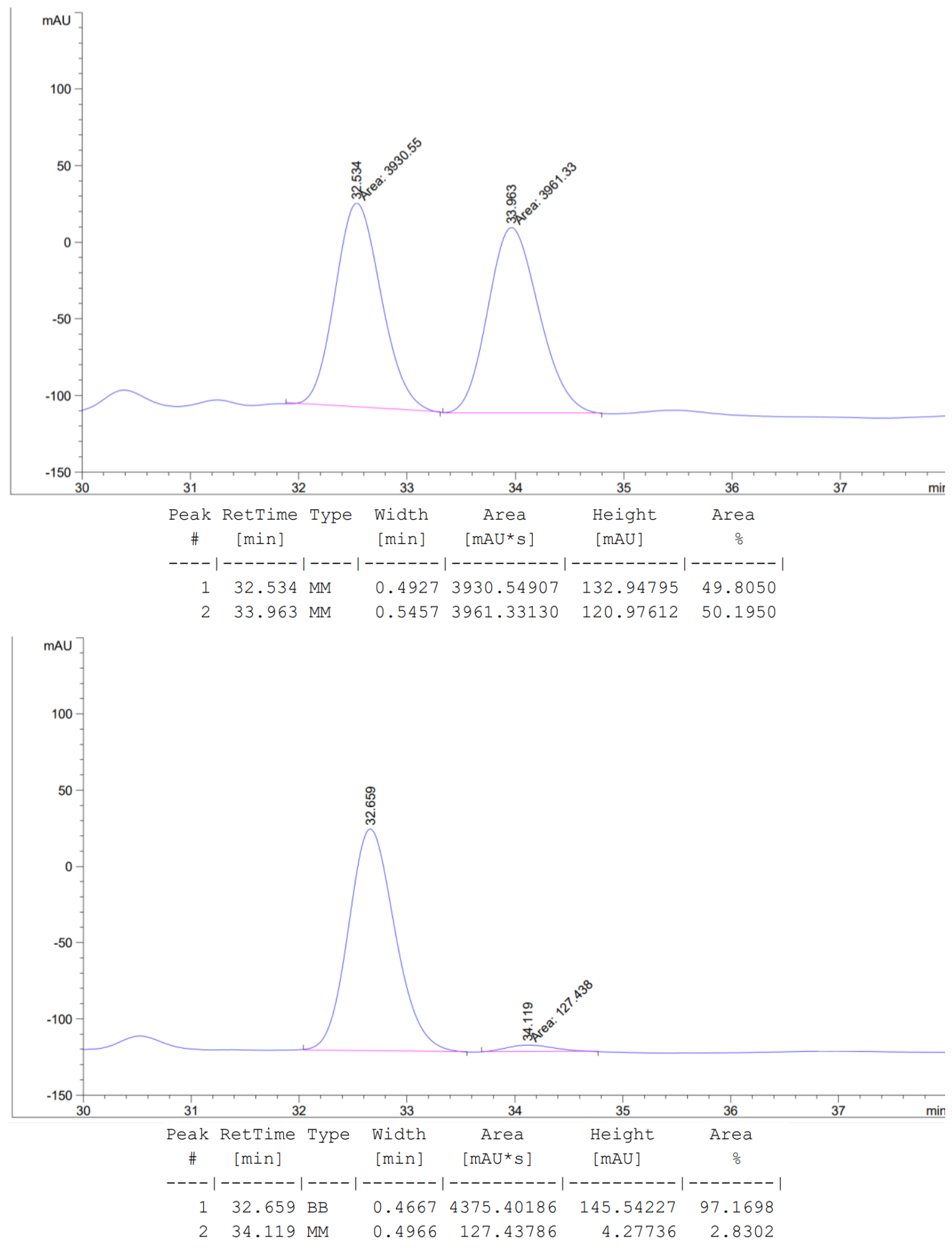
tert-butyl 4-(5-((3S,4S)-4-(benzhydryloxy)-3-hydroxy-4-methylhex-5-en-1-yl)pyrimidin-2yl)piperazine-1-carboxylate (4dd)

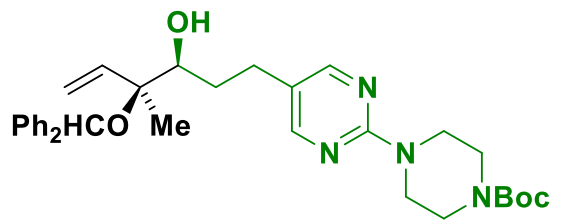

Aldehyde oxidation level: Aldehyde $3 \mathrm{dd}(64.0 \mathrm{mg}, 0.2 \mathrm{mmol})$ was subjected to standard reaction conditions $\left(75^{\circ} \mathrm{C}, 24 \mathrm{~h}\right)$. Upon flash column chromatography $\left(\mathrm{SiO}_{2}, 25: 75\right.$ EtOAc:hexanes), the title compound $4 \mathbf{d d}$ was obtained as a purple oil in $63 \%$ yield $(70.4 \mathrm{mg}, 0.126 \mathrm{mmol}, 10: 1 \mathrm{dr}, 91 \%$ ee).

$\operatorname{TLC}\left(\mathrm{SiO}_{2}\right) \mathrm{R}_{\mathrm{f}}=0.3$ (40:60 EtOAc:hexanes)

${ }^{1} \mathrm{H}$ NMR $\left(500 \mathrm{MHz}, \mathrm{CDCl}_{3}\right) \delta: 8.18(\mathrm{~s}, 2 \mathrm{H}), 7.33-7.28(\mathrm{~m}, 4 \mathrm{H}), 7.28-7.17(\mathrm{~m}, 6 \mathrm{H}), 5.68(\mathrm{dd}, J=$ $17.6,10.9 \mathrm{~Hz}, 1 \mathrm{H}), 5.50(\mathrm{~s}, 1 \mathrm{H}), 5.30-5.21(\mathrm{~m}, 2 \mathrm{H}), 3.76(\mathrm{t}, J=5.1 \mathrm{~Hz}, 4 \mathrm{H}), 3.57(\mathrm{dt}, J=10.4,2.6$ $\mathrm{Hz}, 1 \mathrm{H}), 3.49(\mathrm{t}, J=5.2 \mathrm{~Hz}, 4 \mathrm{H}), 2.74(\mathrm{dd}, J=3.2,1.5 \mathrm{~Hz}, 1 \mathrm{H}), 2.73-2.68(\mathrm{~m}, 1 \mathrm{H}), 2.49(\mathrm{dt}, J=14.2$, $8.3 \mathrm{~Hz}, 1 \mathrm{H}), 2.30-2.18(\mathrm{~m}, 1 \mathrm{H}), 1.59-1.52(\mathrm{~m}, 1 \mathrm{H}), 1.49(\mathrm{~s}, 9 \mathrm{H}), 1.12(\mathrm{~s}, 3 \mathrm{H})$.

${ }^{13} \mathrm{C}$ NMR $\left(125 \mathrm{MHz}, \mathrm{CDCl}_{3}\right) \delta: 160.9,157.8,155.0,144.6,144.1,140.3,128.5,128.4,127.2,127.2$, $126.9,126.7,123.1,118.7,83.1,80.0,76.7,76.2,43.9,32.5,28.6,26.5,16.0$.

HRMS $(\mathrm{Na}+, m / z)$ for $\mathrm{C}_{33} \mathrm{H}_{42} \mathrm{~N}_{4} \mathrm{O}_{4}$ : calcd. = 581.3098; found $=581.3099$.

FTIR (neat): 2985, 2360, 1683, 1494, 1418, 1262, 1167, 1084, $939 \mathrm{~cm}^{-1}$

HPLC: (Two connected Chiralcel columns OD-H and AD-H, Hexane:2-PrOH = 97:03, $0.5 \mathrm{~mL} / \mathrm{min}$, $230 \mathrm{~nm})$

$[\alpha]_{D}^{24}=+29.0^{\circ}\left(\mathrm{c}=0.5, \mathrm{CHCl}_{3}\right)$. 

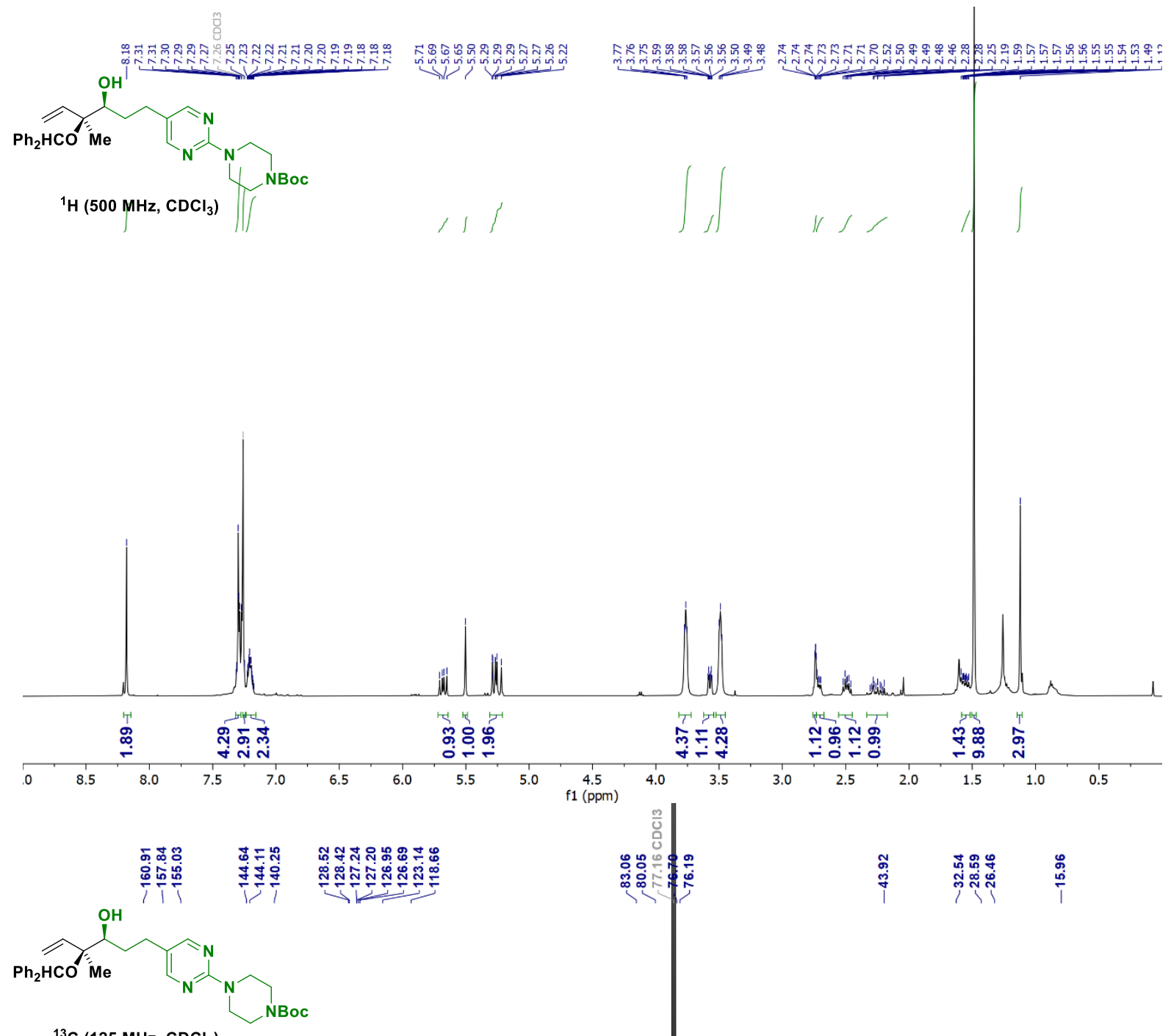

${ }^{13} \mathrm{C}\left(125 \mathrm{MHz}, \mathrm{CDCl}_{3}\right)$

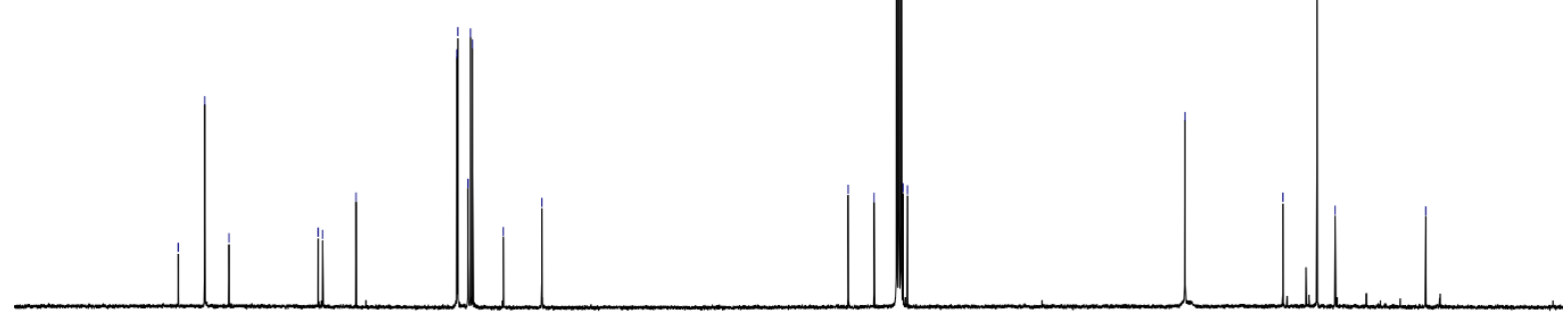

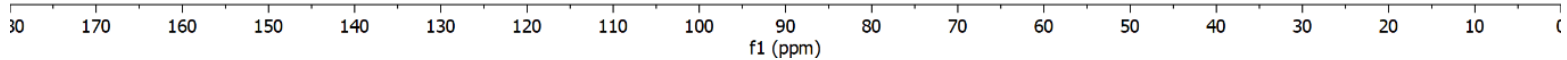




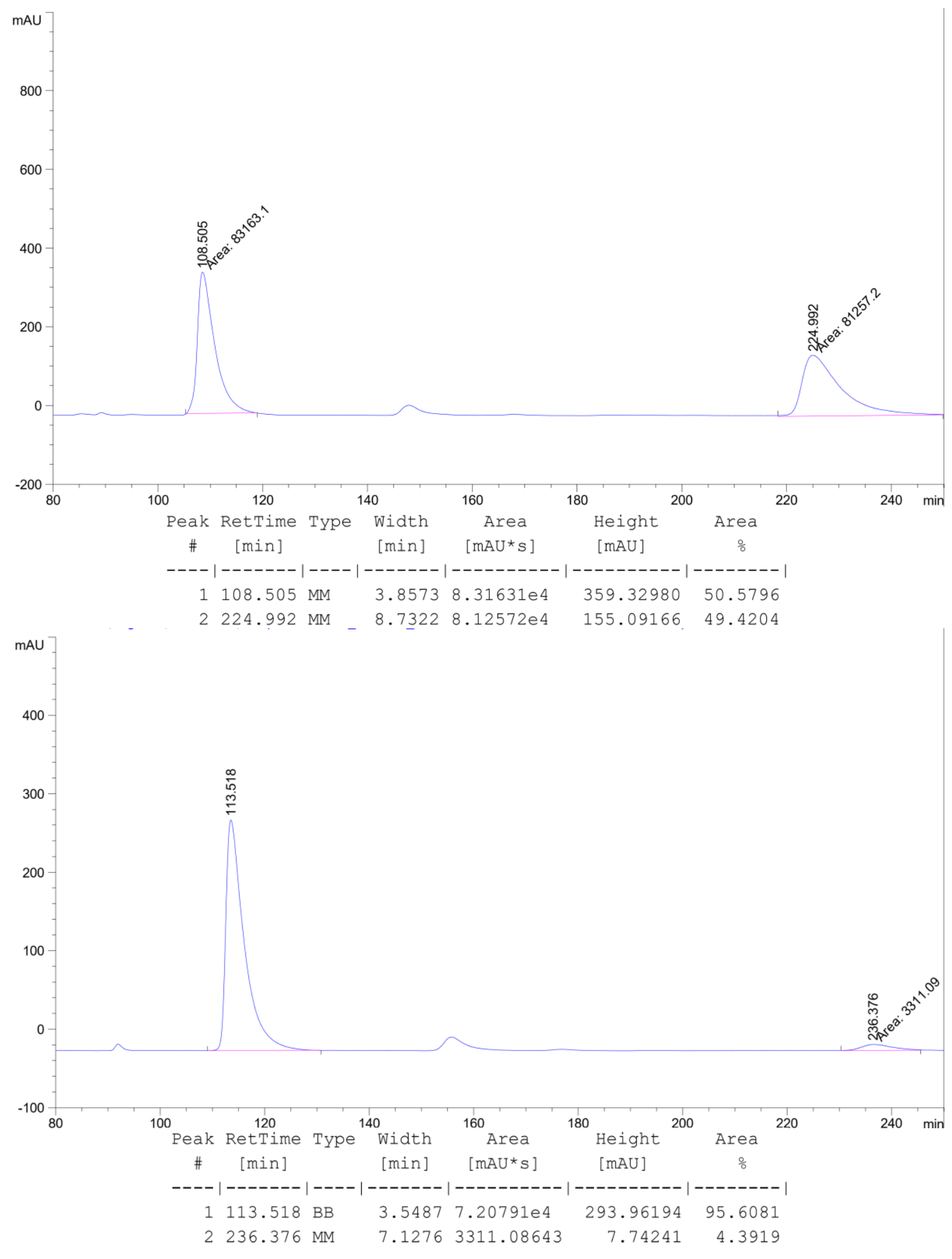


(5-((3S,4S)-4-(benzhydryloxy)-3-hydroxy-4-methylhex-5-en-1-yl)-2-methylphenyl)(5-(4fluorophenyl)thiophen-2-yl)methanone (4ee)

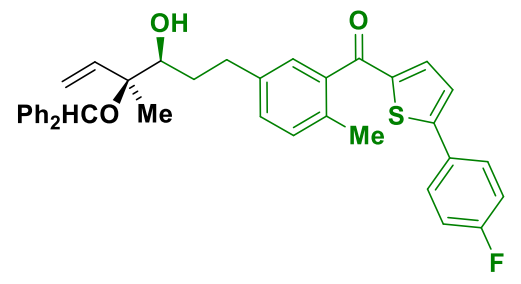

Aldehyde oxidation level: Aldehyde $3 e e(70.4 \mathrm{mg}, 0.2 \mathrm{mmol})$ was subjected to standard reaction conditions $\left(75^{\circ} \mathrm{C}, 24 \mathrm{~h}\right.$ ). Upon flash column chromatography ( $\mathrm{SiO}_{2}, 10: 90$ EtOAc:hexanes), the title compound 4ee was obtained as a yellow solid in $61 \%$ yield $(72.0 \mathrm{mg}, 0.122 \mathrm{mmol}, 8: 1 \mathrm{dr}$, $89 \%$ ee).

$\operatorname{TLC}\left(\mathrm{SiO}_{2}\right) \mathrm{R}_{\mathrm{f}}=0.33$ (20:80 EtOAc:hexanes)

${ }^{1} \mathrm{H} \mathrm{NMR}\left(500 \mathrm{MHz}, \mathrm{CDCl}_{3}\right) \delta: 7.64-7.60(\mathrm{~m}, 2 \mathrm{H}), 7.35(\mathrm{~d}, J=4.0 \mathrm{~Hz}, 1 \mathrm{H}), 7.31-7.27(\mathrm{~m}, 5 \mathrm{H}), 7.26$ $-7.17(\mathrm{~m}, 8 \mathrm{H}), 7.10(\mathrm{t}, J=8.6 \mathrm{~Hz}, 2 \mathrm{H}), 5.68(\mathrm{dd}, J=17.6,10.9 \mathrm{~Hz}, 1 \mathrm{H}), 5.50(\mathrm{~s}, 1 \mathrm{H}), 5.28(\mathrm{~s}, 1 \mathrm{H})$, $5.27-5.20(\mathrm{~m}, 2 \mathrm{H}), 3.60(\mathrm{dt}, J=10.6,2.5 \mathrm{~Hz}, 1 \mathrm{H}), 2.94(\mathrm{ddd}, J=14.3,9.8,4.8 \mathrm{~Hz}, 1 \mathrm{H}), 2.74-2.70$ (m, 1H), 2.65 (ddd, J = 13.9, 9.1, 7.1 Hz, 1H), $2.35(\mathrm{~s}, 3 \mathrm{H}), 1.75-1.66(\mathrm{~m}, 1 \mathrm{H}), 1.60$ (ddd, J = 14.3, 9.5, $4.5 \mathrm{~Hz}, 1 \mathrm{H}), 1.12(\mathrm{~s}, 3 \mathrm{H})$.

${ }^{13} \mathrm{C}$ NMR $\left(125 \mathrm{MHz}, \mathrm{CDCl}_{3}\right) \delta: 190.5,163.4(\mathrm{~d}, J=249.9 \mathrm{~Hz}), 152.7,144.7,144.2,143.8,140.3$, 139.3, 138.4, 136.6, 134.0, 131.2, 130.7, 129.8 (d, $J=3.5 \mathrm{~Hz}), 128.5,128.4,128.3$ (d, $J=8.2 \mathrm{~Hz}$ ), $127.2,127.2,126.9,126.7,124.1,118.6,116.4$ (d, $J=22.0 \mathrm{~Hz}), 83.1,76.7,76.6,53.6,32.9,32.4$, $19.4,16.1$.

${ }^{19} \mathrm{~F} \mathrm{NMR}\left(470 \mathrm{MHz}, \mathrm{CDCl}_{3}\right) \delta:-111.50--111.90(\mathrm{~m})$

HRMS $(\mathrm{Na}+, m / z)$ for $\mathrm{C}_{38} \mathrm{H}_{35} \mathrm{FO}_{3} \mathrm{~S}$ : calcd. $=613.2183$; found $=613.2191$.

FTIR (neat): 3005, 2359, 1635, 1441, 1275, 1084, 810, $764 \mathrm{~cm}^{-1}$

HPLC: (Two connected Chiralcel AD-H columns, Hexane:2-PrOH =95:05, $1.0 \mathrm{~mL} / \mathrm{min}, 210 \mathrm{~nm}$ ) $[\alpha]_{D}^{24}=+26.5^{\circ}\left(\mathrm{c}=0.34, \mathrm{CHCl}_{3}\right)$. 

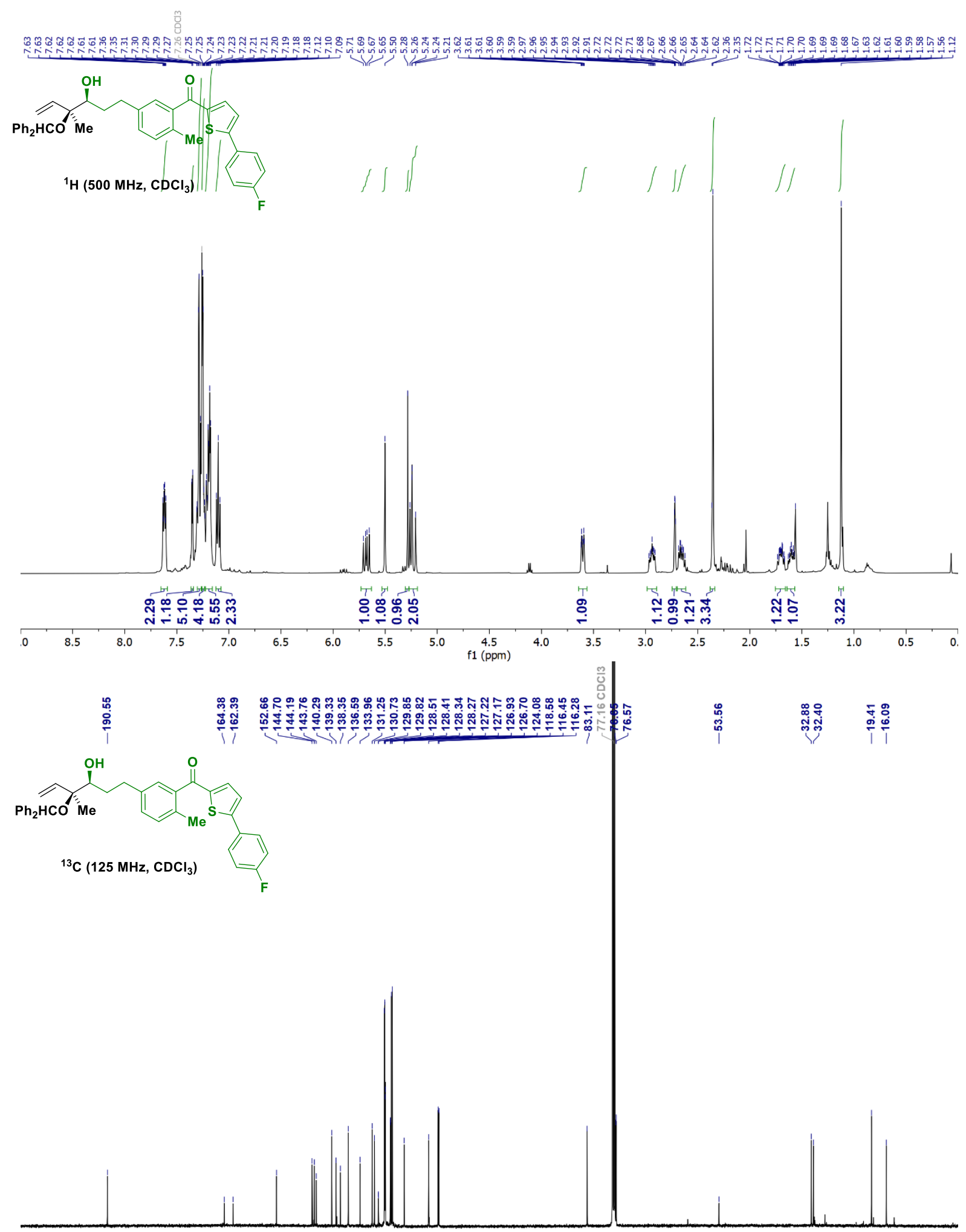

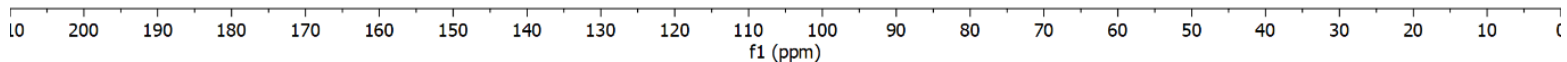




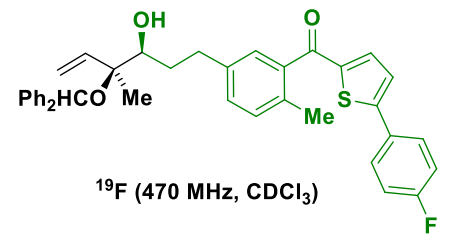

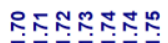

FFFFFFF

ii

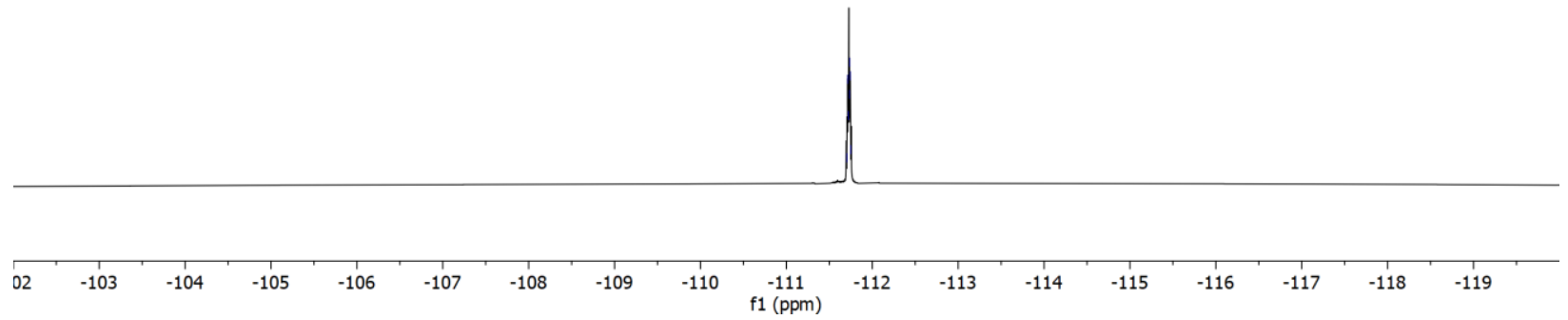




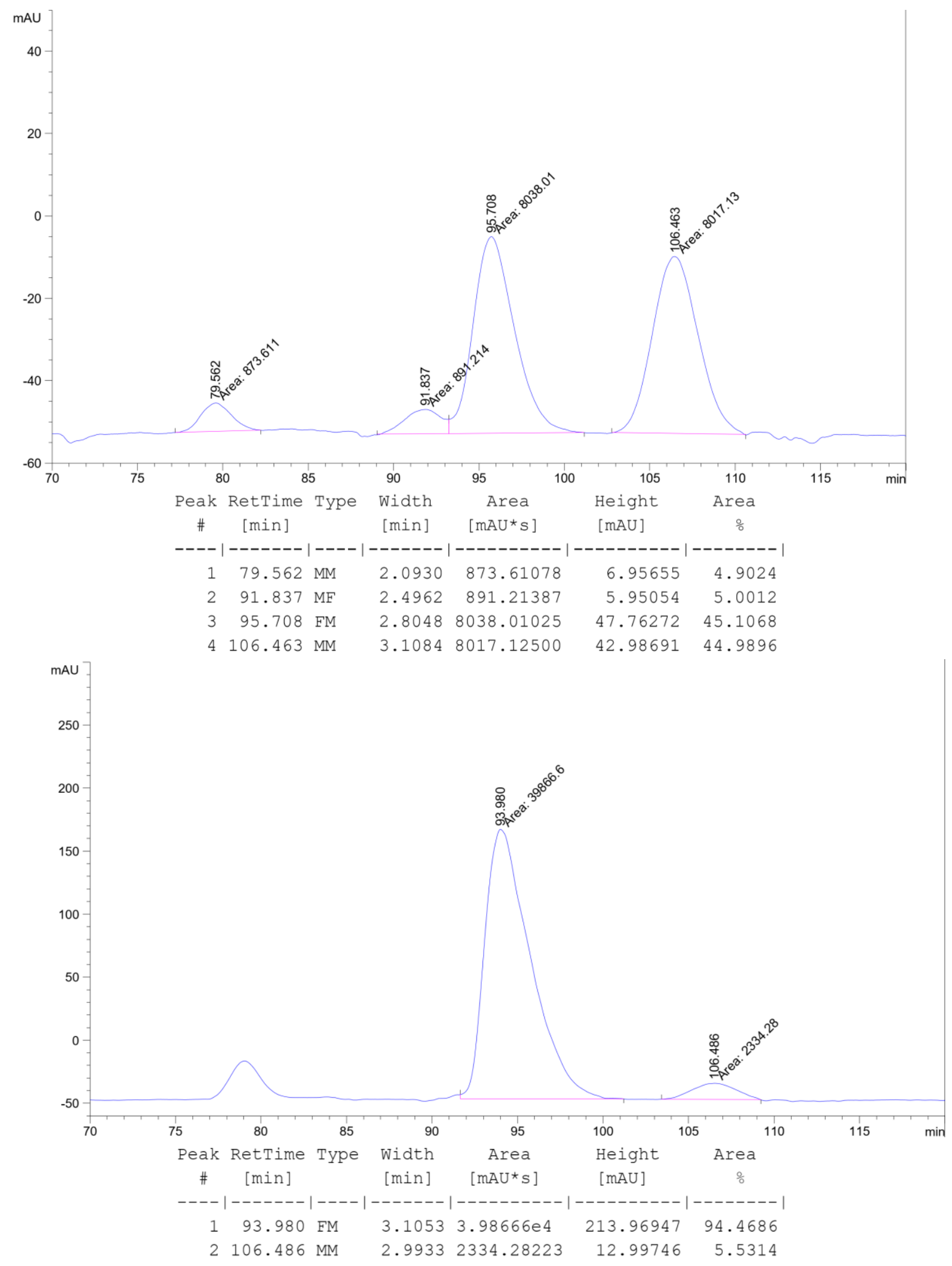




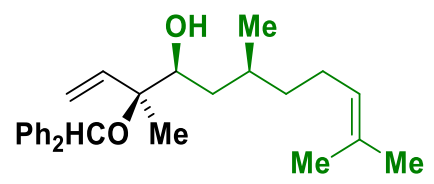

Aldehyde oxidation level: Aldehyde $3 \mathrm{ff}(31.0 \mathrm{mg}, 0.2 \mathrm{mmol})$ was subjected to standard reaction conditions $\left(75^{\circ} \mathrm{C}, 24 \mathrm{~h}\right)$. Upon flash column chromatography $\left(\mathrm{SiO}_{2}, 2: 98 \mathrm{EtOAc}\right.$ :hexanes $)$, the title compound $4 \mathrm{ff}$ was obtained as a colorless oil in $76 \%$ yield $(60.0 \mathrm{mg}, 0.152 \mathrm{mmol}, 6: 1 \mathrm{dr})$.

$\operatorname{TLC}\left(\mathrm{SiO}_{2}\right) \mathrm{R}_{\mathrm{f}}=0.35$ (5:95 EtOAc:hexanes)

${ }^{1} \mathbf{H}$ NMR $\left(500 \mathrm{MHz}_{2} \mathrm{CDCl}_{3}\right) \delta: 7.24-7.17(\mathrm{~m}, 3 \mathrm{H}), 7.15-7.12(\mathrm{~m}, 5 \mathrm{H}), 7.10-7.04(\mathrm{~m}, 2 \mathrm{H}), 5.58$ $(\mathrm{dd}, J=17.6,11.0 \mathrm{~Hz}, 1 \mathrm{H}), 5.40(\mathrm{~d}, J=2.9 \mathrm{~Hz}, 1 \mathrm{H}), 5.18-5.10(\mathrm{~m}, 2 \mathrm{H}), 4.98(\mathrm{t}, J=7.2 \mathrm{~Hz}, 1 \mathrm{H}), 3.58$ $(\mathrm{dt}, J=10.6,2.3 \mathrm{~Hz}, 1 \mathrm{H}), 1.86$ (hept, $J=7.5 \mathrm{~Hz}, 2 \mathrm{H}), 1.64-1.57(\mathrm{~m}, 1 \mathrm{H}), 1.55(\mathrm{~s}, 3 \mathrm{H}), 1.47(\mathrm{~s}, 3 \mathrm{H})$, $1.28-1.14(\mathrm{~m}, 2 \mathrm{H}), 1.11-1.03(\mathrm{~m}, 1 \mathrm{H}), 1.00(\mathrm{~s}, 3 \mathrm{H}), 0.98-0.92(\mathrm{~m}, 1 \mathrm{H}), 0.76(\mathrm{~d}, J=6.6 \mathrm{~Hz}, 3 \mathrm{H})$.

${ }^{13} \mathrm{C} \mathrm{NMR}\left(125 \mathrm{MHz}, \mathrm{CDCl}_{3}\right) \delta: 144.9,144.3,140.6,131.2,128.5,128.4,127.2,127.1,127.0,126.7$, $125.0,118.3,83.4,76.6,75.1,38.4,38.2,29.5,25.9,25.8,19.0,17.8,15.9$.

HRMS $(\mathrm{Na}+, m / z)$ for $\mathrm{C}_{27} \mathrm{H}_{36} \mathrm{O}_{2}$ : calcd. = 415.2608; found $=415.2615$.

FTIR (neat): 3350, 2916, 2358, 1635, 1263, 1024, $747 \mathrm{~cm}^{-1}$

$[\alpha]_{D}^{24}=+21.4^{\circ}\left(\mathrm{c}=0.14, \mathrm{CHCl}_{3}\right)$. 


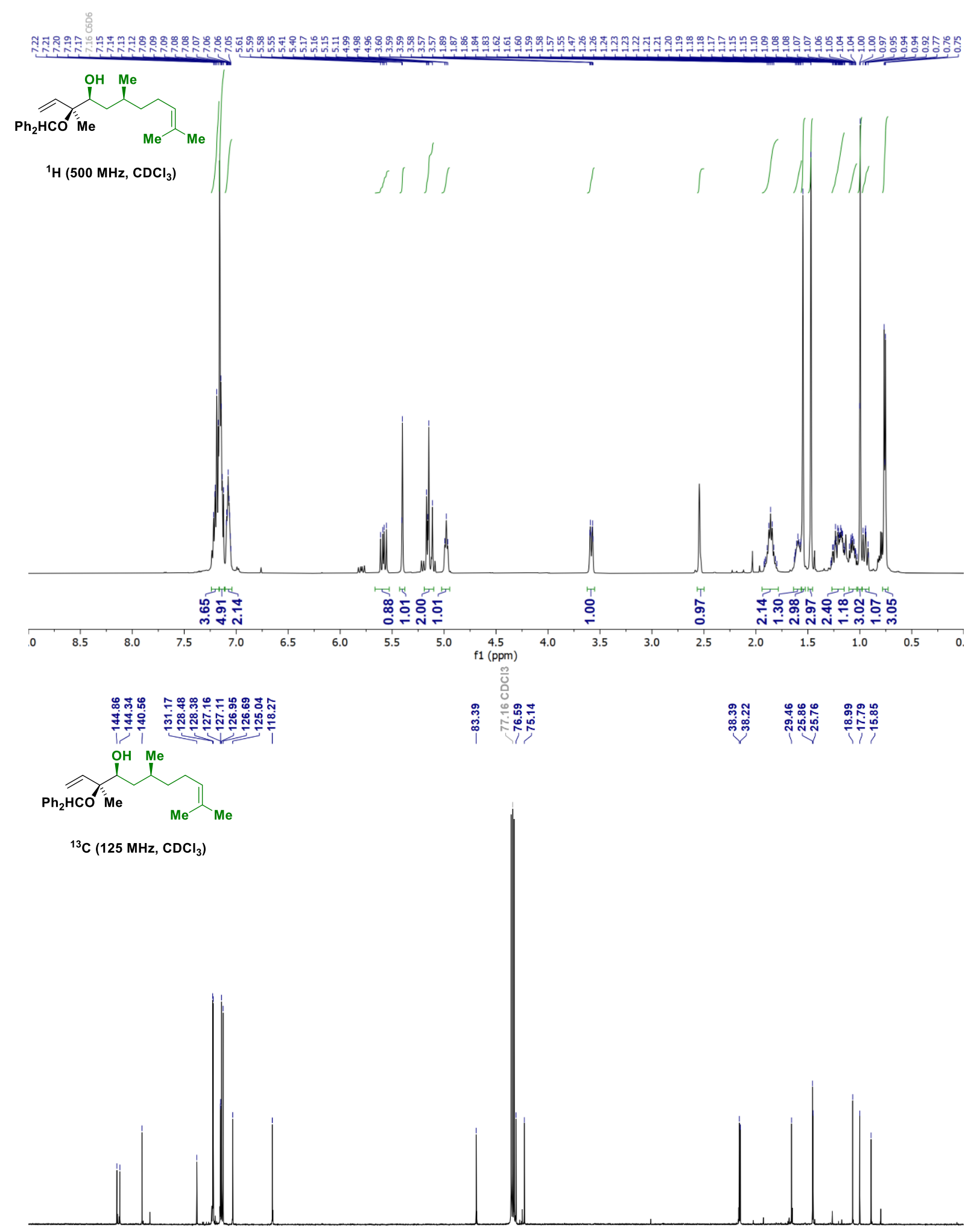

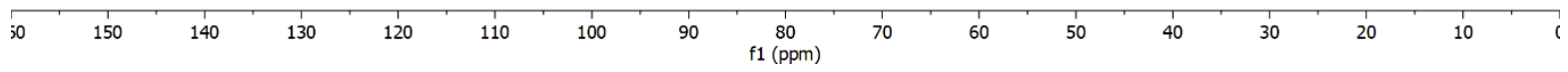




\section{(3R,4R,6S)-3-(benzhydryloxy)-3,6,10-trimethylundeca-1,9-dien-4-ol (iso-4ff)}

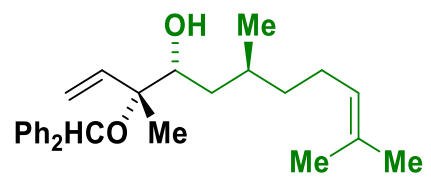

Aldehyde oxidation level: Aldehyde $3 \mathrm{ff}(31.0 \mathrm{mg}, 0.2 \mathrm{mmol})$ was subjected to standard reaction conditions, (S)-BINAP was used $\left(75{ }^{\circ} \mathrm{C}, 24 \mathrm{~h}\right)$. Upon flash column chromatography $\left(\mathrm{SiO}_{2}, 2: 98\right.$ EtOAc:hexanes), the title compound iso-4ff was obtained as a colorless oil in $70 \%$ yield $(55.0 \mathrm{mg}$, $0.14 \mathrm{mmol}, 11: 1 \mathrm{dr})$.

$\operatorname{TLC}\left(\mathrm{SiO}_{2}\right) \mathrm{R}_{\mathrm{f}}=0.35$ (5:95 EtOAc:hexanes)

${ }^{1} \mathbf{H}$ NMR $\left(500 \mathrm{MHz}, \mathrm{CDCl}_{3}\right) \delta: 7.34-7.27(\mathrm{~m}, 3 \mathrm{H}), 7.26-7.23(\mathrm{~m}, 5 \mathrm{H}), 7.22-7.14(\mathrm{~m}, 2 \mathrm{H}), 5.67$ $(\mathrm{dd}, J=17.6,10.9 \mathrm{~Hz}, 1 \mathrm{H}), 5.50(\mathrm{~s}, 1 \mathrm{H}), 5.28-5.21(\mathrm{~m}, 2 \mathrm{H}), 5.08(\mathrm{tt}, J=7.2,1.7 \mathrm{~Hz}, 1 \mathrm{H}), 3.68(\mathrm{dt}$, $J=9.9,2.3 \mathrm{~Hz}, 1 \mathrm{H}), 2.68(\mathrm{t}, J=2.1 \mathrm{~Hz}, 1 \mathrm{H}), 2.14(\mathrm{~s}, 2 \mathrm{H}), 1.98(\mathrm{dt}, J=14.7,7.5 \mathrm{~Hz}, 1 \mathrm{H}), 1.88(\mathrm{dq}, J=$ 14.3, 7.1 Hz, 1H), $1.66(\mathrm{~s}, 3 \mathrm{H}), 1.57(\mathrm{~s}, 3 \mathrm{H}), 1.47-1.37(\mathrm{~m}, 1 \mathrm{H}), 1.25(\mathrm{ddt}, J=12.4,8.6,2.0 \mathrm{~Hz}, 1 \mathrm{H})$, $1.18(\mathrm{dd}, J=9.8,4.8 \mathrm{~Hz}, 1 \mathrm{H}), 1.09(\mathrm{~s}, 3 \mathrm{H}), 1.07-0.98(\mathrm{~m}, 1 \mathrm{H}), 0.91(\mathrm{~d}, J=6.7 \mathrm{~Hz}, 3 \mathrm{H})$.

${ }^{13} \mathrm{C}$ NMR $\left(125 \mathrm{MHz}, \mathrm{CDCl}_{3}\right) \delta: 144.8,144.3,140.5,131.2,128.5,128.4,127.2,127.1,127.0,126.7$, $125.1,118.4,83.5,76.6,75.6,38.4,36.1,30.1,25.9,25.5,20.9,17.8,15.7$.

HRMS $(M+, m / z)$ for $\mathrm{C}_{27} \mathrm{H}_{36} \mathrm{O}_{2}$ : calcd. $=415.2608$; found $=415.2615$.

FTIR (neat): 3350, 2916, 2358, 1635, 1263, 1024, $747 \mathrm{~cm}^{-1}$

$[\alpha]_{D}^{24}=-15.2^{\circ}\left(\mathrm{c}=0.33, \mathrm{CHCl}_{3}\right)$. 


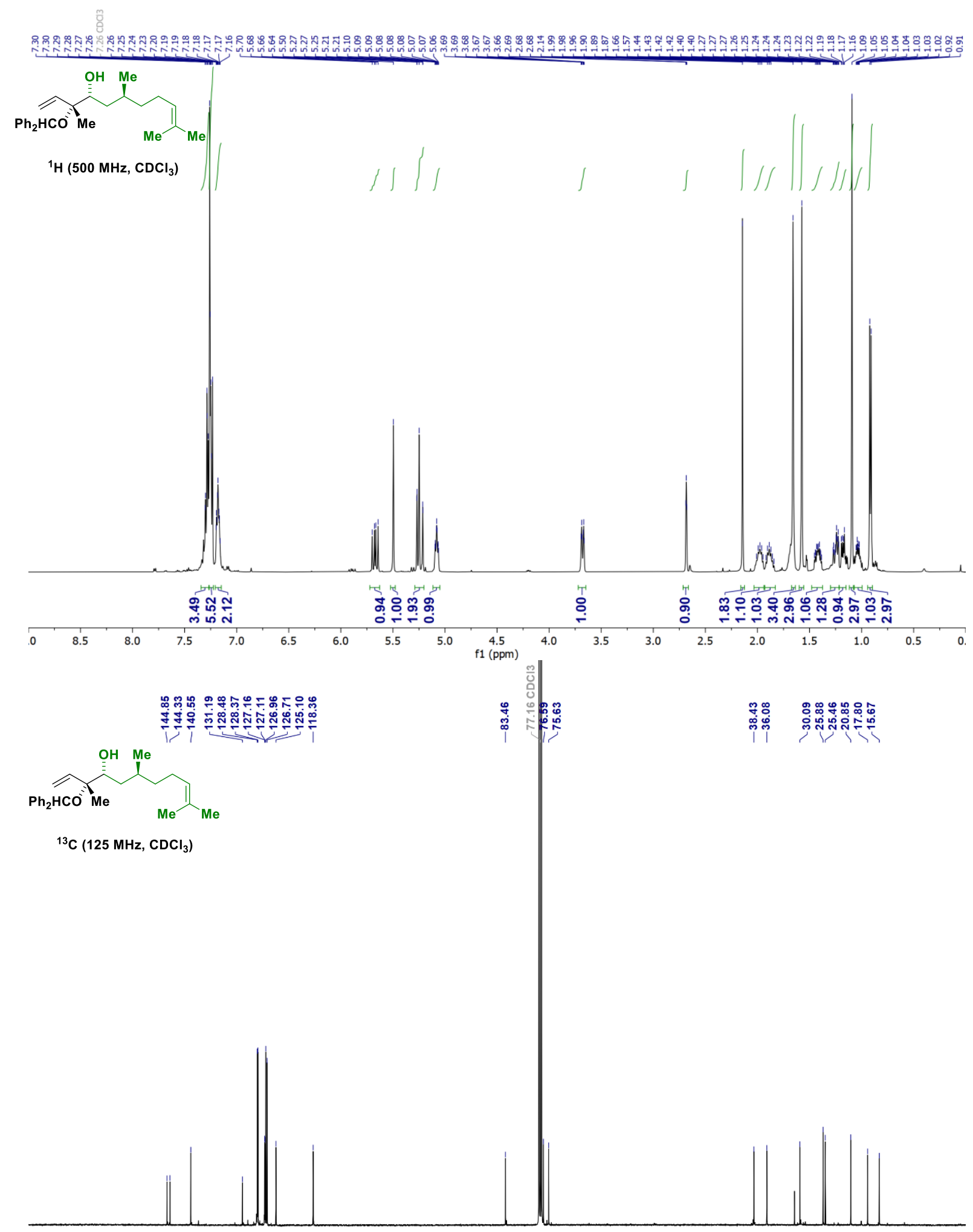

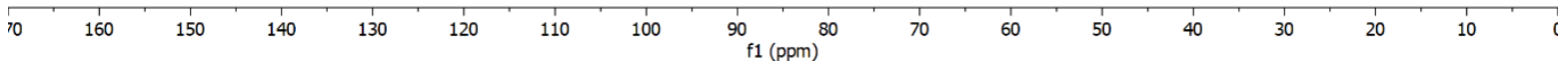


(3S,4S,6R)-3-(benzhydryloxy)-6-((4-methoxybenzyl)oxy)-3-methylhept-1-en-4-ol (4gg)

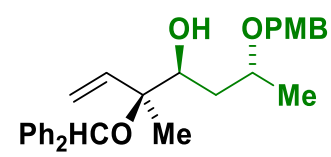

Aldehyde oxidation level: Aldehyde $3 \mathrm{gg}(41.6 \mathrm{mg}, 0.2 \mathrm{mmol})$ was subjected to standard reaction conditions $\left(75^{\circ} \mathrm{C}, 24 \mathrm{~h}\right.$ ). Upon flash column chromatography $\left(\mathrm{SiO}_{2}, 10: 90\right.$ EtOAc:hexanes), the title compound $\mathbf{4 g g}$ was obtained as a colorless oil in $77 \%$ yield ( $68.8 \mathrm{mg}, 0.154 \mathrm{mmol}, 10: 1 \mathrm{dr}$ ).

$\operatorname{TLC}\left(\mathrm{SiO}_{2}\right) \mathrm{R}_{\mathrm{f}}=0.35$ (20:80 EtOAc:hexanes)

${ }^{1} \mathbf{H}$ NMR $\left(500 \mathrm{MHz}, \mathrm{CDCl}_{3}\right) \delta: 7.34-7.27(\mathrm{~m}, 8 \mathrm{H}), 7.25(\mathrm{~d}, J=8.6 \mathrm{~Hz}, 2 \mathrm{H}), 7.19(\mathrm{td}, J=7.0,4.4 \mathrm{~Hz}$, $2 \mathrm{H}), 6.86(\mathrm{~d}, J=8.6 \mathrm{~Hz}, 2 \mathrm{H}), 5.76(\mathrm{dd}, J=18.2,10.4 \mathrm{~Hz}, 1 \mathrm{H}), 5.54(\mathrm{~s}, 1 \mathrm{H}), 5.29-5.23(\mathrm{~m}, 2 \mathrm{H}), 4.52$ $(\mathrm{d}, J=11.0 \mathrm{~Hz}, 1 \mathrm{H}), 4.39(\mathrm{~d}, J=11.1 \mathrm{~Hz}, 1 \mathrm{H}), 3.79(\mathrm{~s}, 3 \mathrm{H}), 3.76-3.71(\mathrm{~m}, 2 \mathrm{H}), 3.31(\mathrm{~d}, J=2.0 \mathrm{~Hz}$, $1 \mathrm{H}), 1.69$ (ddd, $J=14.4,6.5,2.4 \mathrm{~Hz}, 1 \mathrm{H}), 1.66-1.60(\mathrm{~m}, 1 \mathrm{H}), 1.21(\mathrm{~d}, J=6.1 \mathrm{~Hz}, 3 \mathrm{H}), 1.19(\mathrm{~s}, 3 \mathrm{H})$.

${ }^{13} \mathrm{C}$ NMR $\left(125 \mathrm{MHz}, \mathrm{CDCl}_{3}\right) \delta: 159.2,144.9,144.4,140.0,130.6,129.3,128.3,128.2,127.0,126.9$, $126.7,126.7,117.7,113.8,82.5,76.5,76.1,74.6,70.0,55.3,37.9,19.5,17.7$.

HRMS $(\mathrm{Na}+, m / z)$ for $\mathrm{C}_{29} \mathrm{H}_{34} \mathrm{O}_{4}$ : calcd. = 469.2349; found $=469.2351$.

FTIR (neat): 3567, 2966, 2361, 1512, 1259, 1082, 1033, $749 \mathrm{~cm}^{-1}$

$[\alpha]_{D}^{24}=-25.0^{\circ}\left(\mathrm{c}=0.28, \mathrm{CHCl}_{3}\right)$. 


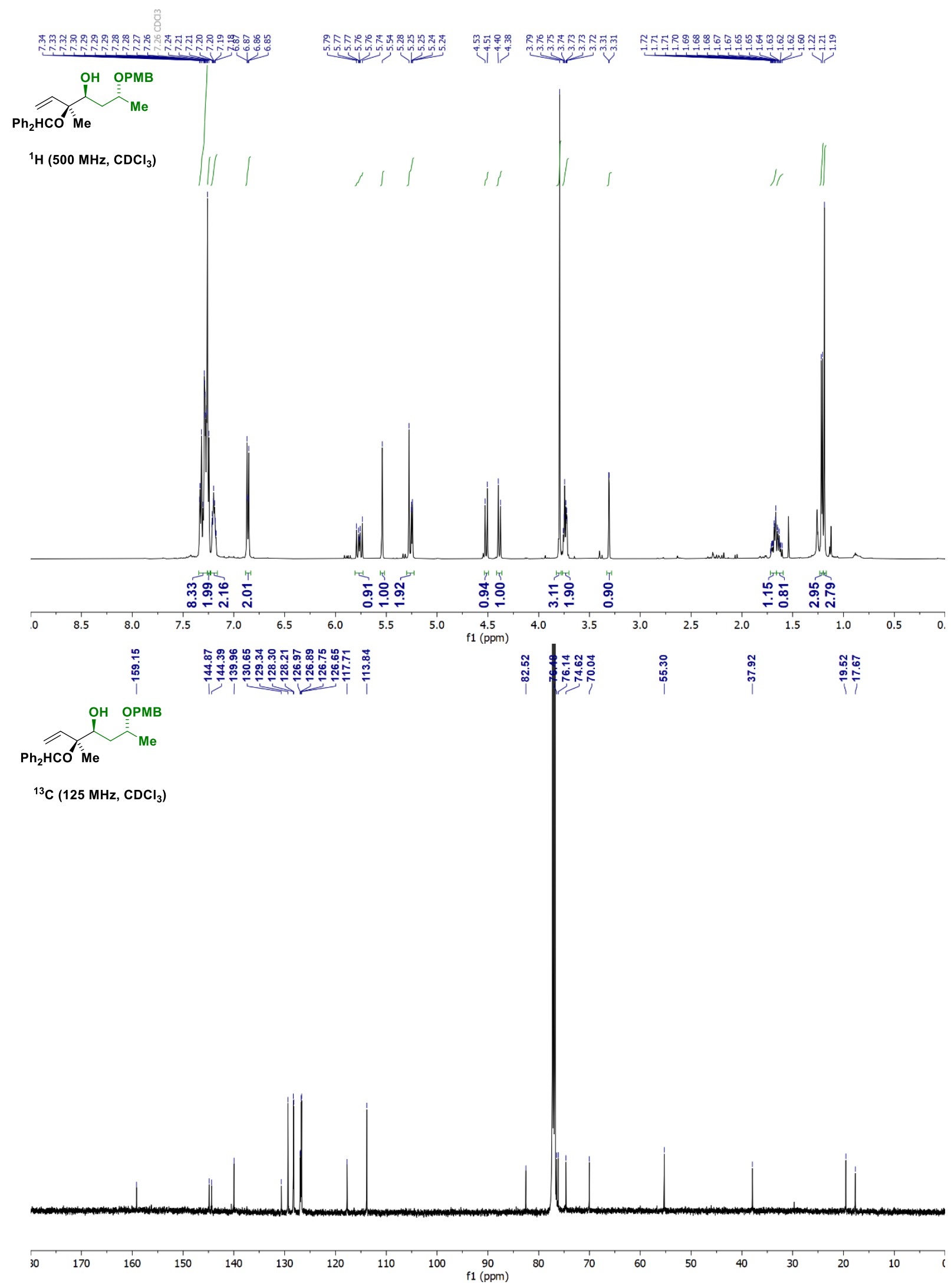


(3R,4R,6R)-3-(benzhydryloxy)-6-((4-methoxybenzyl)oxy)-3-methylhept-1-en-4-ol (iso-4gg)

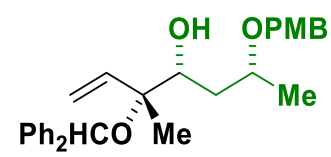

Aldehyde oxidation level: Aldehyde $3 \mathrm{gg}(41.6 \mathrm{mg}, 0.2 \mathrm{mmol})$ was subjected to standard reaction conditions, (S)-BINAP was used $\left(75^{\circ} \mathrm{C}, 24 \mathrm{~h}\right)$. Upon flash column chromatography $\left(\mathrm{SiO}_{2}, 10: 90\right.$ EtOAc:hexanes), the title compound iso-4gg was obtained as a colorless oil in $70 \%$ yield $(60.7 \mathrm{mg}$, $0.14 \mathrm{mmol}, 13: 1 \mathrm{dr}$ ).

$\operatorname{TLC}\left(\mathrm{SiO}_{2}\right) \mathrm{R}_{\mathrm{f}}=0.35$ (20:80 EtOAc:hexanes)

${ }^{1} \mathbf{H}$ NMR $\left(500 \mathrm{MHz}, \mathrm{CDCl}_{3}\right) \delta: 7.35-7.26(\mathrm{~m}, 10 \mathrm{H}), 7.24-7.16(\mathrm{~m}, 2 \mathrm{H}), 6.89-6.85(\mathrm{~m}, 2 \mathrm{H}), 5.76$ $(\mathrm{dd}, J=17.5,11.1 \mathrm{~Hz}, 1 \mathrm{H}), 5.53(\mathrm{~s}, 1 \mathrm{H}), 5.32-5.22(\mathrm{~m}, 2 \mathrm{H}), 4.53(\mathrm{~d}, J=11.2 \mathrm{~Hz}, 1 \mathrm{H}), 4.41(\mathrm{~d}, J=$ $11.3 \mathrm{~Hz}, 1 \mathrm{H}$ ), 3.92 (dd, $J=10.7,2.3 \mathrm{~Hz}, 1 \mathrm{H}), 3.85-3.80(\mathrm{~m}, 1 \mathrm{H}), 3.80(\mathrm{~s}, 3 \mathrm{H}), 2.77$ (dd, $J=2.7,1.4$ $\mathrm{Hz}, 1 \mathrm{H}), 1.62$ (dd, $J=14.2,8.9 \mathrm{~Hz}, 1 \mathrm{H}), 1.42$ (ddd, $J=13.9,10.5,3.2 \mathrm{~Hz}, 1 \mathrm{H}), 1.20(\mathrm{~d}, J=6.2 \mathrm{~Hz}$, $3 \mathrm{H}), 1.14(\mathrm{~s}, 3 \mathrm{H})$.

${ }^{13} \mathrm{C}$ NMR $\left(125 \mathrm{MHz}, \mathrm{CDCl}_{3}\right) \delta: 159.2,144.9,144.4,140.3,131.3,129.3,128.5,128.4,127.1,127.1$, $127.0,126.8,118.3,113.9,82.9,76.6,73.8,72.3,70.7,55.4,38.6,20.3,16.6$.

HRMS $(\mathrm{Na}+, m / z)$ for $\mathrm{C}_{29} \mathrm{H}_{34} \mathrm{O}_{4}$ : calcd. = 469.2349; found $=469.2351$.

FTIR (neat): 3567, 2966, 2361, 1512, 1259, 1082, 1033, $749 \mathrm{~cm}^{-1}$

$[\alpha]_{D}^{24}=+16.7^{\circ}\left(\mathrm{c}=0.36, \mathrm{CHCl}_{3}\right)$. 


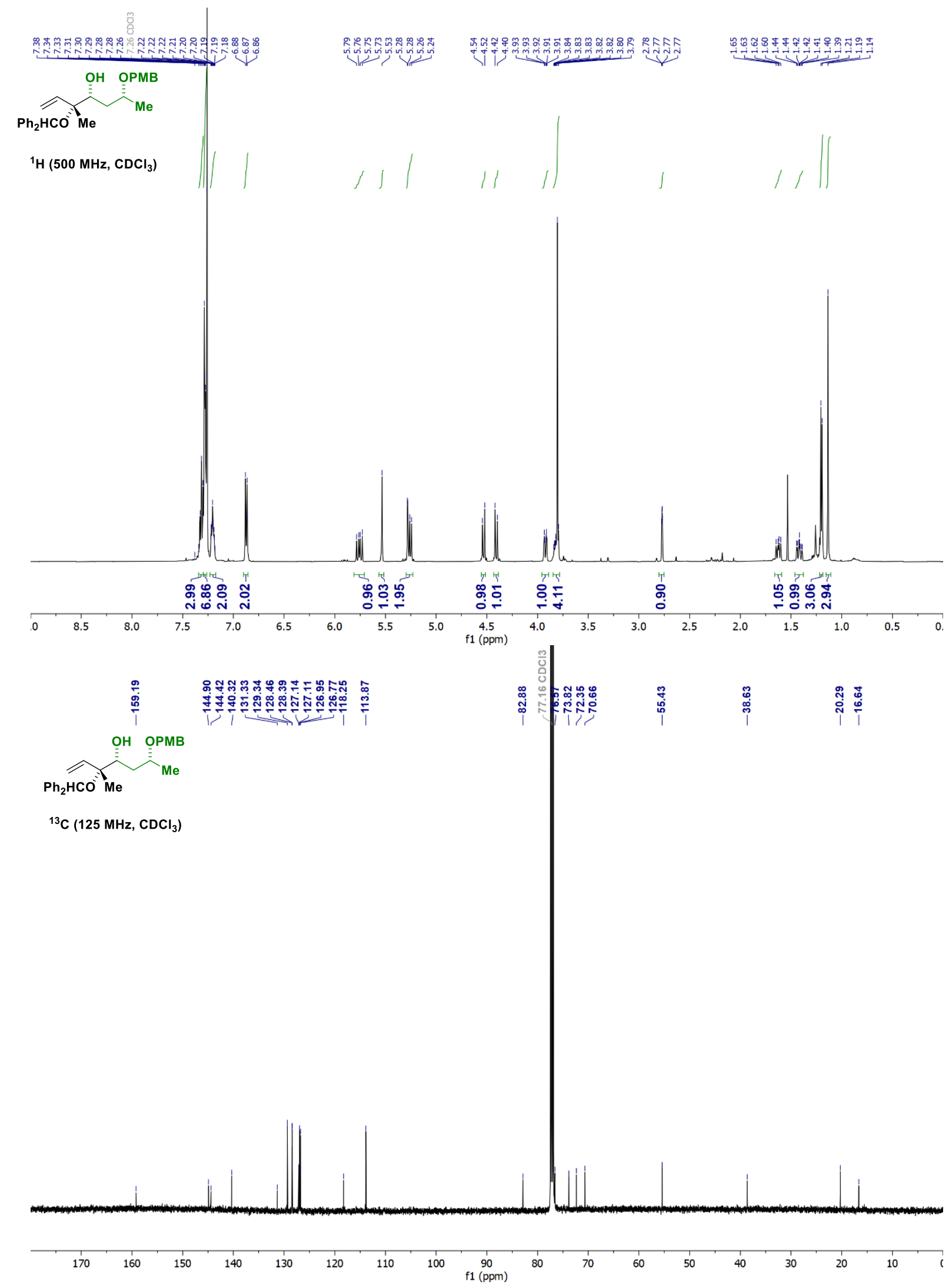


1-((1S,3S)-3-((2S,3S)-3-(benzhydryloxy)-2-hydroxy-3-methylpent-4-en-1-yl)-2,2dimethylcyclobutyl)ethan-1-one (4hh)

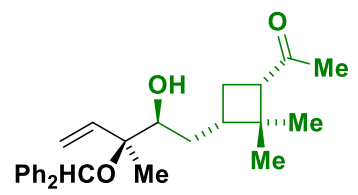

Aldehyde oxidation level: Aldehyde $3 \mathrm{hh}(33.6 \mathrm{mg}, 0.2 \mathrm{mmol})$ was subjected to standard reaction conditions $\left(75^{\circ} \mathrm{C}, 24 \mathrm{~h}\right)$. Upon flash column chromatography ( $\mathrm{SiO}_{2}, 10: 90$ EtOAc:hexanes), the title compound $4 \mathrm{hh}$ was obtained as a white solid in $72 \%$ yield ( $58.5 \mathrm{mg}, 0.144 \mathrm{mmol}, 13: 1 \mathrm{dr}$ ).

$\operatorname{TLC}\left(\mathrm{SiO}_{2}\right) \mathrm{R}_{\mathrm{f}}=0.30$ (20:80 EtOAc:hexanes)

${ }^{1} \mathbf{H}$ NMR $\left(500 \mathrm{MHz}, \mathrm{CDCl}_{3}\right) \delta: 7.32-7.24(\mathrm{~m}, 8 \mathrm{H}), 7.19(\mathrm{ddt}, J=8.7,5.5,2.9 \mathrm{~Hz}, 2 \mathrm{H}), 5.69(\mathrm{dd}, J=$ 17.6, $10.9 \mathrm{~Hz}, 1 \mathrm{H}), 5.49(\mathrm{~s}, 1 \mathrm{H}), 5.30-5.22(\mathrm{~m}, 2 \mathrm{H}), 3.50(\mathrm{dt}, J=11.3,2.2 \mathrm{~Hz}, 1 \mathrm{H}), 2.81(\mathrm{dd}, J=$ 10.3, 7.6 Hz, 1H), 2.60 (dd, $J=3.1,1.6 \mathrm{~Hz}, 1 \mathrm{H}), 2.22$ (dtd, $J=10.6,8.6,5.9 \mathrm{~Hz}, 1 \mathrm{H}), 2.01(\mathrm{~s}, 3 \mathrm{H})$, $1.97-1.85(\mathrm{~m}, 2 \mathrm{H}), 1.46(\mathrm{ddt}, J=12.4,8.7,1.8 \mathrm{~Hz}, 1 \mathrm{H}), 1.32(\mathrm{~s}, 3 \mathrm{H}), 1.19$ (ddd, $J=14.1,10.8,5.8$ $\mathrm{Hz}, 1 \mathrm{H}), 1.09(\mathrm{~s}, 3 \mathrm{H}), 0.81(\mathrm{~s}, 3 \mathrm{H})$.

${ }^{13} \mathrm{C}$ NMR $\left(125 \mathrm{MHz}, \mathrm{CDCl}_{3}\right) \delta: 208.2,144.8,144.3,140.3,128.5,128.4,127.2,127.2,126.9,126.7$, $118.6,83.3,76.8,76.7,54.6,43.7,39.2,31.8,31.1,30.3,24.1,17.4,15.8$.

HRMS $(\mathrm{Na}+, m / z)$ for $\mathrm{C}_{27} \mathrm{H}_{34} \mathrm{O}_{3}$ : calcd. $=429.2400 ;$ found $=429.2410$.

FTIR (neat): 3005, 2989, 2359, 2342, 1700, 1454, 1262, $749 \mathrm{~cm}^{-1}$

$[\alpha]_{D}^{24}=+60.0^{\circ}\left(\mathrm{c}=0.35, \mathrm{CHCl}_{3}\right)$.

MP: $[80-85]^{\circ} \mathrm{C}$ 


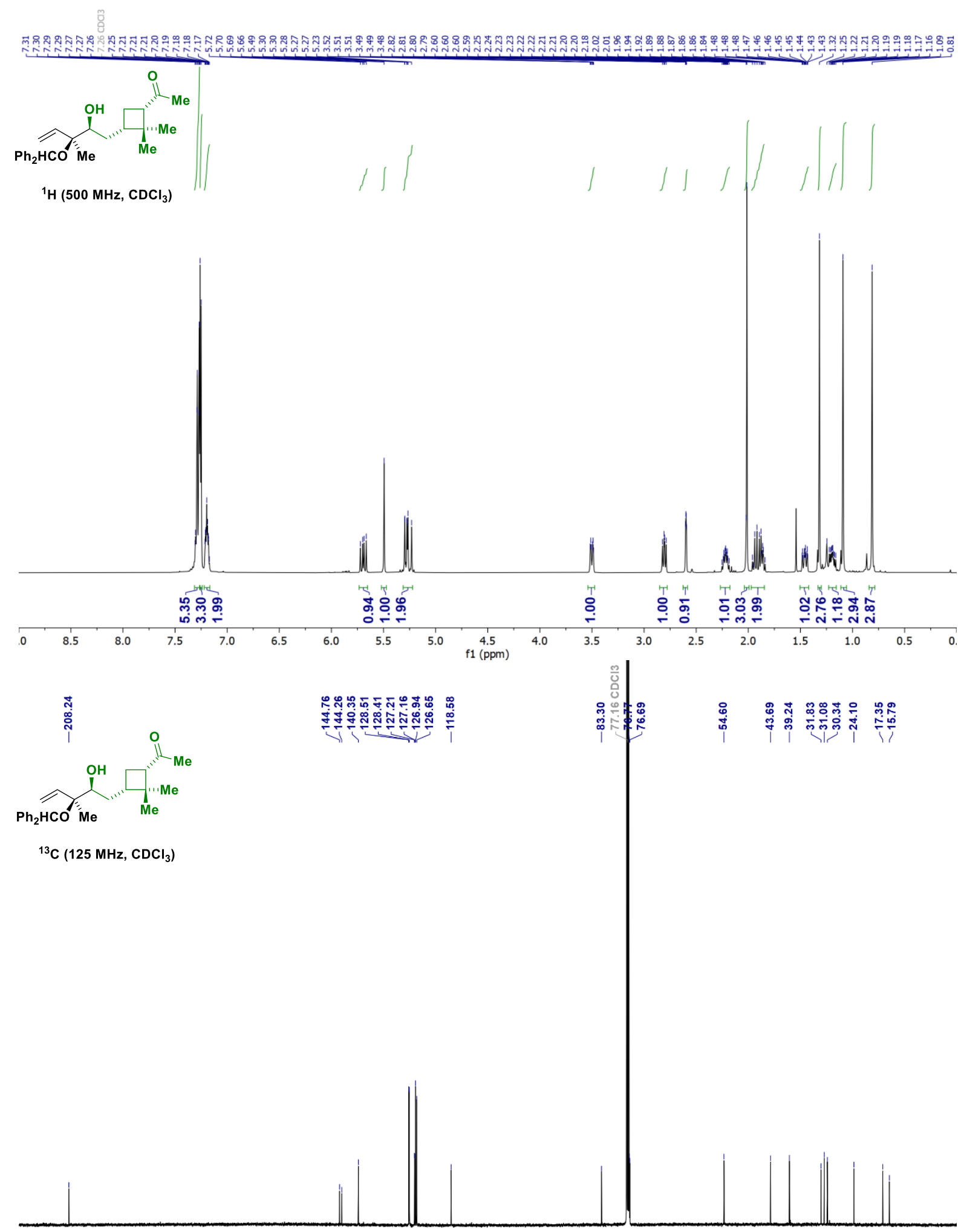

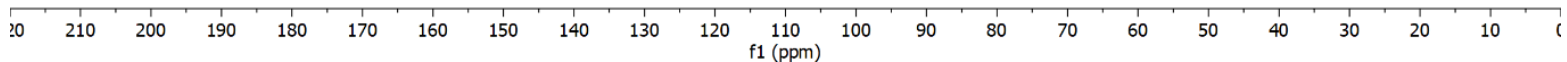


1-((1S,3S)-3-((2R,3R)-3-(benzhydryloxy)-2-hydroxy-3-methylpent-4-en-1-yl)-2,2dimethylcyclobutyl)ethan-1-one (iso-4hh)

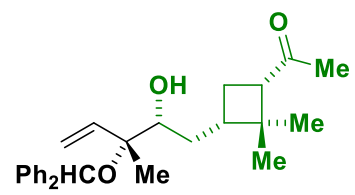

Aldehyde oxidation level: Aldehyde $3 \mathrm{hh}(33.6 \mathrm{mg}, 0.2 \mathrm{mmol}$ ) was subjected to standard reaction conditions, (S)-BINAP was used $\left(75^{\circ} \mathrm{C}, 24 \mathrm{~h}\right)$. Upon flash column chromatography $\left(\mathrm{SiO}_{2}, 10: 90\right.$ EtOAc:hexanes), the title compound iso-4hh was obtained as a colorless oil in $66 \%$ yield ( $53.5 \mathrm{mg}$, $0.132 \mathrm{mmol}, 8: 1 \mathrm{dr}$ ).

$\operatorname{TLC}\left(\mathrm{SiO}_{2}\right) \mathrm{R}_{\mathrm{f}}=0.30$ (20:80 EtOAc:hexanes)

${ }^{1} \mathbf{H}$ NMR $\left(500 \mathrm{MHz}, \mathrm{CDCl}_{3}\right) \delta: 7.33-7.24(\mathrm{~m}, 8 \mathrm{H}), 7.23-7.16(\mathrm{~m}, 2 \mathrm{H}), 5.67(\mathrm{dd}, J=17.6,10.9 \mathrm{~Hz}$, $1 \mathrm{H}), 5.51(\mathrm{~s}, 1 \mathrm{H}), 5.32-5.22(\mathrm{~m}, 2 \mathrm{H}), 3.50(\mathrm{dt}, J=10.2,2.4 \mathrm{~Hz}, 1 \mathrm{H}), 2.83(\mathrm{dd}, J=10.2,7.4 \mathrm{~Hz}, 1 \mathrm{H})$, $2.69(\mathrm{t}, J=2.1 \mathrm{~Hz}, 1 \mathrm{H}), 2.21(\mathrm{tdd}, J=10.3,8.0,5.0 \mathrm{~Hz}, 1 \mathrm{H}), 2.02(\mathrm{~s}, 3 \mathrm{H}), 1.87(\mathrm{dt}, J=11.0,7.7 \mathrm{~Hz}$, $1 \mathrm{H}), 1.80(\mathrm{q}, J=10.7 \mathrm{~Hz}, 1 \mathrm{H}), 1.29(\mathrm{~s}, 3 \mathrm{H}), 1.27-1.17(\mathrm{~m}, 2 \mathrm{H}), 1.09(\mathrm{~s}, 3 \mathrm{H}), 0.79(\mathrm{~s}, 3 \mathrm{H})$.

${ }^{13} \mathrm{C}$ NMR $\left(125 \mathrm{MHz}, \mathrm{CDCl}_{3}\right) \delta: 208.3,144.7,144.2,140.3,128.5,128.4,127.2,127.2,126.9,126.7$, $118.7,83.2$, 76.6, 75.0, 54.3, 43.3, 39.2, 30.9, 30.3, 30.2, 22.8, 17.5, 15.7 .

HRMS $(\mathrm{Na}+, m / z)$ for $\mathrm{C}_{27} \mathrm{H}_{34} \mathrm{O}_{3}$ : calcd. $=429.2400$; found $=429.2410$.

FTIR (neat): 3005, 2989, 2359, 2342, 1700, 1454, 1262, $749 \mathrm{~cm}^{-1}$

$[\alpha]_{D}^{24}=-1.6^{\circ}\left(\mathrm{c}=0.32, \mathrm{CHCl}_{3}\right)$. 


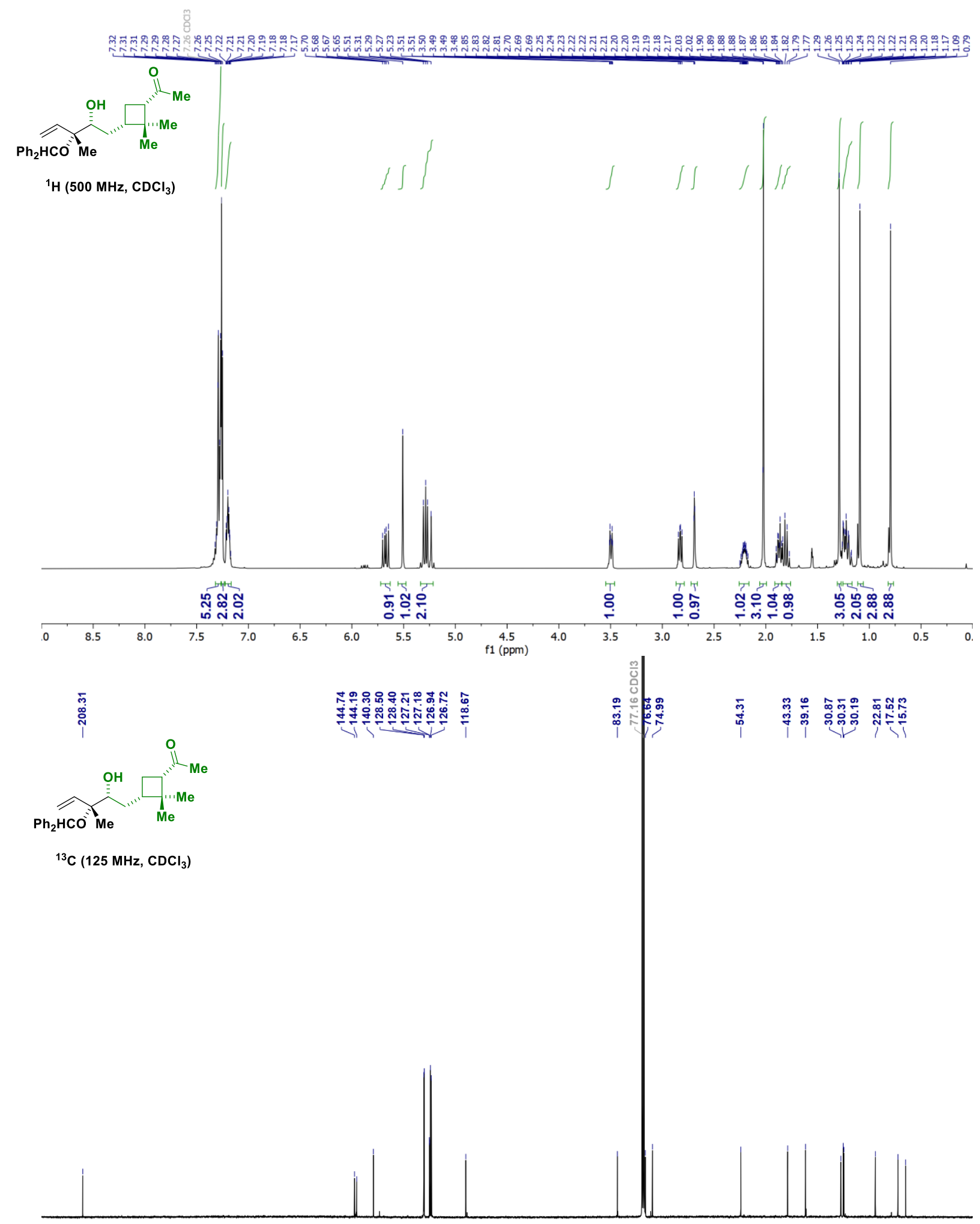

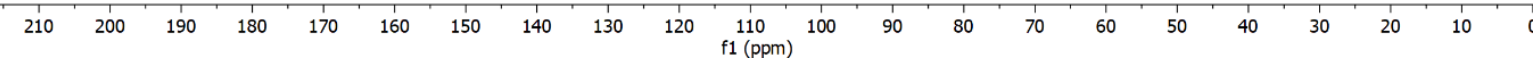


1-((1S,3R)-3-((2S,3S)-3-(benzhydryloxy)-2-hydroxy-3-methylpent-4-en-1-yl)-2,2dimethylcyclopropyl)propan-2-one (4ii)

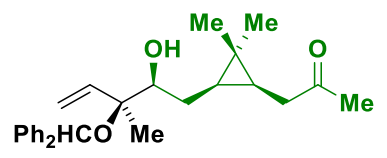

Aldehyde oxidation level: Aldehyde $3 \mathrm{ii}(33.6 \mathrm{mg}, 0.2 \mathrm{mmol})$ was subjected to standard reaction conditions $\left(75^{\circ} \mathrm{C}, 24 \mathrm{~h}\right)$. Upon flash column chromatography ( $\mathrm{SiO}_{2}, 10: 90$ EtOAc:hexanes), the title compound $4 \mathrm{ii}$ was obtained as a colorless oil in $59 \%$ yield ( $48.0 \mathrm{mg}, 0.118 \mathrm{mmol}, 12: 1 \mathrm{dr}$ ).

$\operatorname{TLC}\left(\mathrm{SiO}_{2}\right) \mathrm{R}_{\mathrm{f}}=0.30$ (15:85 EtOAc:hexanes)

${ }^{1} \mathbf{H}$ NMR $\left(500 \mathrm{MHz}, \mathrm{CDCl}_{3}\right) \delta: 7.34-7.30(\mathrm{~m}, 3 \mathrm{H}), 7.30-7.26(\mathrm{~m}, 5 \mathrm{H}), 7.20(\mathrm{ddt}, J=9.4,5.9,2.2$ $\mathrm{Hz}, 2 \mathrm{H}), 5.72(\mathrm{dd}, J=17.6,11.0 \mathrm{~Hz}, 1 \mathrm{H}), 5.52(\mathrm{~s}, 1 \mathrm{H}), 5.30-5.23(\mathrm{~m}, 2 \mathrm{H}), 3.62(\mathrm{dd}, J=10.0,2.1$ $\mathrm{Hz}, 1 \mathrm{H}), 2.56(\mathrm{~s}, 1 \mathrm{H}), 2.38(\mathrm{dd}, J=17.4,6.3 \mathrm{~Hz}, 1 \mathrm{H}), 2.26(\mathrm{dd}, J=17.3,6.9 \mathrm{~Hz}, 1 \mathrm{H}), 2.15(\mathrm{~s}, 3 \mathrm{H})$, $1.32(\mathrm{ddt}, J=14.4,6.8,2.0 \mathrm{~Hz}, 1 \mathrm{H}), 1.27-1.17(\mathrm{~m}, 1 \mathrm{H}), 1.13(\mathrm{~s}, 3 \mathrm{H}), 1.10(\mathrm{~s}, 3 \mathrm{H}), 0.90-0.87(\mathrm{~m}$, $2 \mathrm{H}), 0.86(\mathrm{~s}, 3 \mathrm{H})$.

${ }^{13} \mathrm{C}$ NMR (125 MHz, $\left.\mathrm{CDCl}_{3}\right) \delta: 209.7,144.9,144.4,140.4,128.5,128.4,127.2,127.1,126.9,126.7$, $118.4,83.2,78.3,76.7,39.8,29.8,29.1,26.3,23.9,21.7,16.9,16.2,15.2$.

HRMS $(\mathrm{Na}+, m / z)$ for $\mathrm{C}_{27} \mathrm{H}_{34} \mathrm{O}_{3}$ : calcd. $=429.2400$; found $=429.2408$.

FTIR (neat): 3440, 2988, 2360, 1714, 1274, 1264, $733 \mathrm{~cm}^{-1}$

$[\alpha]_{D}^{24}=+20.6^{\circ}\left(\mathrm{c}=0.34, \mathrm{CHCl}_{3}\right)$. 


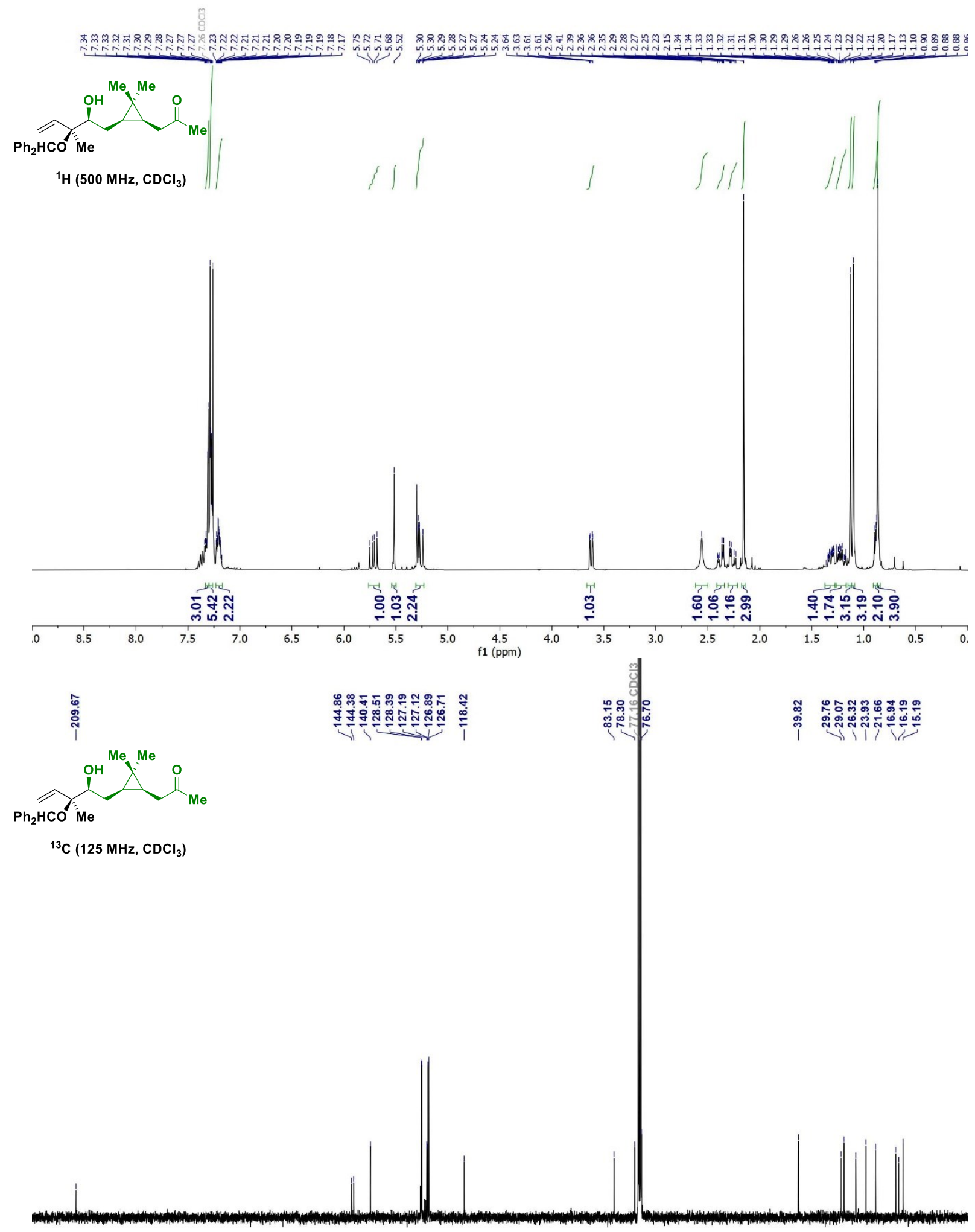

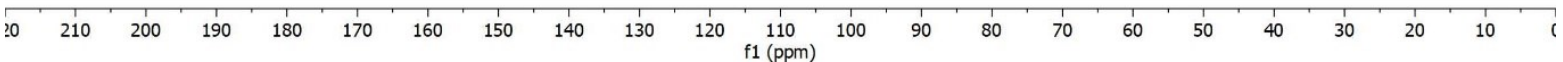


1-((1S,3R)-3-((2R,3R)-3-(benzhydryloxy)-2-hydroxy-3-methylpent-4-en-1-yl)-2,2dimethylcyclopropyl)propan-2-one (iso-4ii)

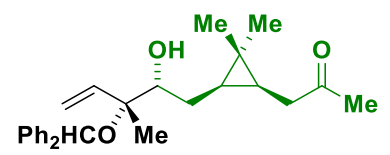

Aldehyde oxidation level: Aldehyde $3 \mathrm{ii}(33.6 \mathrm{mg}, 0.2 \mathrm{mmol})$ was subjected to standard reaction conditions, (S)-BINAP was used $\left(75^{\circ} \mathrm{C}, 24 \mathrm{~h}\right)$. Upon flash column chromatography $\left(\mathrm{SiO}_{2}, 10: 90\right.$ EtOAc:hexanes), the title compound iso-4ii was obtained as a colorless oil in $64 \%$ yield $(52.0 \mathrm{mg}$, $0.128 \mathrm{mmol}, 14: 1 \mathrm{dr})$.

$\operatorname{TLC}\left(\mathrm{SiO}_{2}\right) \mathrm{R}_{\mathrm{f}}=0.30$ (15:85 EtOAc:hexanes)

${ }^{1} \mathbf{H}$ NMR $\left(500 \mathrm{MHz}, \mathrm{CDCl}_{3}\right) \delta: 7.34-7.30(\mathrm{~m}, 3 \mathrm{H}), 7.30-7.26(\mathrm{~m}, 5 \mathrm{H}), 7.20(\mathrm{ddt}, J=10.5,6.2,3.2$ $\mathrm{Hz}, 2 \mathrm{H}), 5.71(\mathrm{dd}, J=17.6,11.0 \mathrm{~Hz}, 1 \mathrm{H}), 5.52(\mathrm{~s}, 1 \mathrm{H}), 5.32-5.23(\mathrm{~m}, 2 \mathrm{H}), 3.60(\mathrm{dt}, J=10.0,2.7 \mathrm{~Hz}$, $1 \mathrm{H}), 2.66(\mathrm{dd}, J=3.2,1.3 \mathrm{~Hz}, 1 \mathrm{H}), 2.34(\mathrm{dd}, J=17.4,6.3 \mathrm{~Hz}, 1 \mathrm{H}), 2.24(\mathrm{dd}, J=17.4,7.4 \mathrm{~Hz}, 1 \mathrm{H})$, $2.14(\mathrm{~s}, 3 \mathrm{H}), 1.32(\mathrm{ddt}, J=14.3,9.6,4.7 \mathrm{~Hz}, 1 \mathrm{H}), 1.27-1.17(\mathrm{~m}, 1 \mathrm{H}), 1.13(\mathrm{~s}, 3 \mathrm{H}), 1.09(\mathrm{~s}, 3 \mathrm{H}), 0.88$ $(\mathrm{s}, 3 \mathrm{H}), 0.87-0.78(\mathrm{~m}, 2 \mathrm{H})$.

${ }^{13} \mathrm{C}$ NMR $\left(125 \mathrm{MHz}, \mathrm{CDCl}_{3}\right) \delta: 209.2,144.8,144.3,140.5,128.5,128.4,127.2,127.1,126.9,126.7$, $118.5,83.1,77.8,76.8,76.7,39.6,29.6,28.8,26.2,23.7,21.3,16.2,15.4$.

HRMS $(\mathrm{Na}+, m / z)$ for $\mathrm{C}_{27} \mathrm{H}_{34} \mathrm{O}_{3}$ : calcd. $=429.2400$; found $=429.2408$.

FTIR (neat): 3440, 2988, 2360, 1714, 1274, 1264, $733 \mathrm{~cm}^{-1}$

$[\alpha]_{D}^{24}=-50.0^{\circ}\left(\mathrm{c}=0.35, \mathrm{CHCl}_{3}\right)$. 


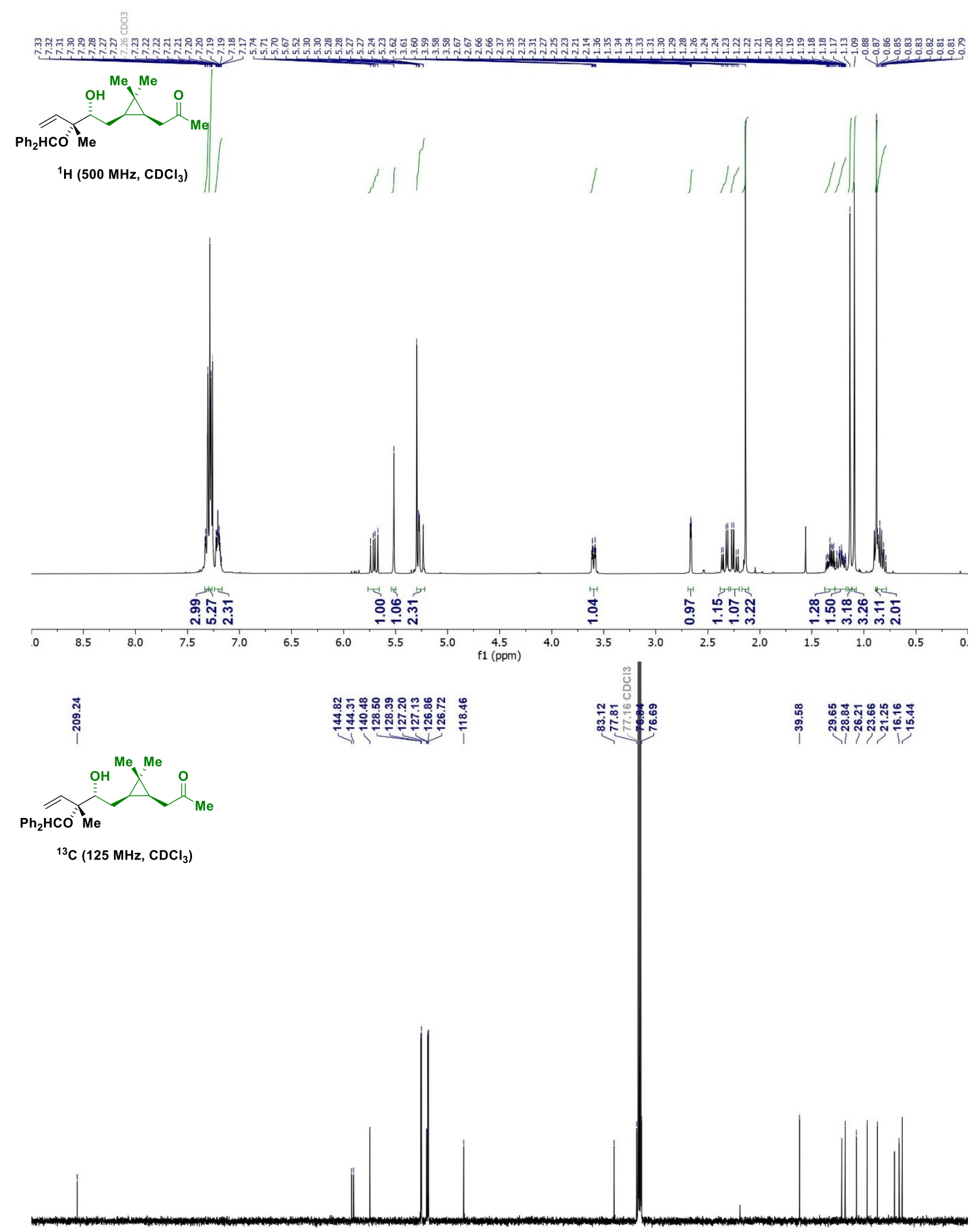

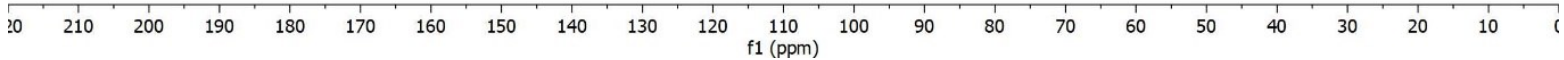


1-(2-((4R,6S)-6-((2S,3S)-3-(benzhydryloxy)-2-hydroxy-3-methylpent-4-en-1-yl)-2,2-dimethyl1,3-dioxan-4-yl)ethyl)-5-(4-fluorophenyl)-2-isopropyl-N,4-diphenyl-1H-pyrrole-3-carboxamide (4jj)

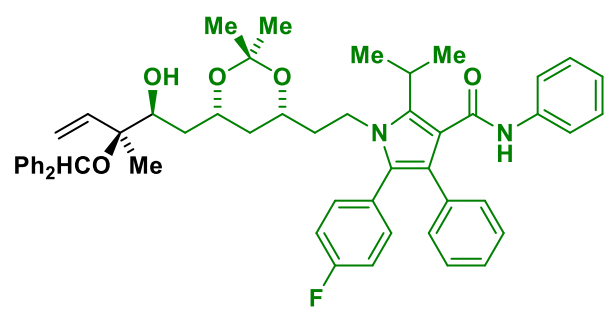

Aldehyde oxidation level: Aldehyde $3 \mathbf{j j}$ (from ref.4)(116.5 mg, $0.2 \mathrm{mmol}$ ) was subjected to standard reaction conditions (10 mol\% catalyst loading, $75^{\circ} \mathrm{C}, 48 \mathrm{~h}$ ). Upon flash column chromatography $\left(\mathrm{SiO}_{2}, 30: 70\right.$ EtOAc:hexanes), the title compound $4 \mathbf{j j}$ was obtained as a yellow oil in $62 \%$ yield ( $101.8 \mathrm{mg}, 0.124 \mathrm{mmol}, 12: 1 \mathrm{dr}$ ).

$\operatorname{TLC}\left(\mathrm{SiO}_{2}\right) \mathrm{R}_{\mathrm{f}}=0.5$ (40:60 EtOAc:hexanes)

${ }^{1} \mathrm{H}$ NMR $\left(500 \mathrm{MHz}, \mathrm{CDCl}_{3}\right) \delta: 7.33(\mathrm{~d}, \mathrm{~J}=7.1 \mathrm{~Hz}, 2 \mathrm{H}), 7.30(\mathrm{~d}, \mathrm{~J}=2.2 \mathrm{~Hz}, 2 \mathrm{H}), 7.27-7.24(\mathrm{~m}, 3 \mathrm{H})$, 7.19 (dtd, J = 13.0, 7.7, 5.7 Hz, 12H), 7.08 (d, J = 8.0 Hz, 2H), 6.99 (td, J = 8.0, 7.4, 4.9 Hz, 3H), 6.87 $(\mathrm{s}, 1 \mathrm{H}), 5.75(\mathrm{dd}, \mathrm{J}=17.6,11.0 \mathrm{~Hz}, 1 \mathrm{H}), 5.54(\mathrm{~s}, 1 \mathrm{H}), 5.31-5.23(\mathrm{~m}, 2 \mathrm{H}), 4.17-4.03(\mathrm{~m}, 2 \mathrm{H}), 4.00$ (dtd, J = 9.1, 6.6, 2.2 Hz, 1H), $3.83(\mathrm{ddd}, \mathrm{J}=14.3,10.1,6.1 \mathrm{~Hz}, 1 \mathrm{H}), 3.72-3.63(\mathrm{~m}, 2 \mathrm{H}), 3.57(\mathrm{~h}, \mathrm{~J}$ $=7.1 \mathrm{~Hz}, 1 \mathrm{H}), 3.15(\mathrm{~s}, 1 \mathrm{H}), 2.05(\mathrm{~s}, 1 \mathrm{H}), 1.71-1.61(\mathrm{~m}, 3 \mathrm{H}), 1.55(\mathrm{~s}, 3 \mathrm{H}), 1.53(\mathrm{~s}, 3 \mathrm{H}), 1.38(\mathrm{~s}, 3 \mathrm{H})$, $1.31(\mathrm{~s}, 3 \mathrm{H}), 1.17(\mathrm{~s}, 3 \mathrm{H})$.

${ }^{13} \mathrm{C}$ NMR $\left(125 \mathrm{MHz}, \mathrm{CDCl}_{3}\right) \delta: 171.3,164.1(\mathrm{~d}, J=196.1 \mathrm{~Hz}), 161.4,144.9,144.3,141.6,139.9$, $138.5,134.8,133.3$ (d, $J=8.0 \mathrm{~Hz}), 132.6,130.6,128.8,128.5,128.4,128.4,128.1,127.1,127.1$, $126.9,126.7,123.6,121.9,119.7,118.2,115.5$ (d, $J=21.4 \mathrm{~Hz}), 115.5,98.7,82.5,76.7,75.4,68.5$, $66.6,60.5,41.0,38.2,37.4,36.1,30.2,26.2,21.9,21.7,20.0,17.5$.

${ }^{19}$ F NMR (470 MHz, $\left.\mathrm{CDCl}_{3}\right) \delta:-113.6$ (tq, J = 8.7, 5.3, 4.3 Hz).

HRMS $(\mathrm{Na}+, m / z)$ for $\mathrm{C}_{53} \mathrm{H}_{57} \mathrm{FN}_{2} \mathrm{O}_{5}$ : calcd. $=843.4144 ;$ found $=843.4147$.

FTIR(neat): 3646, 2246, 1731, 1661, 1525, 1435, 1241, 1156, 1093, 906, $727 \mathrm{~cm}^{-1}$

$[\alpha]_{D}^{24}=+45.0^{\circ}\left(\mathrm{c}=0.2, \mathrm{CHCl}_{3}\right)$. 

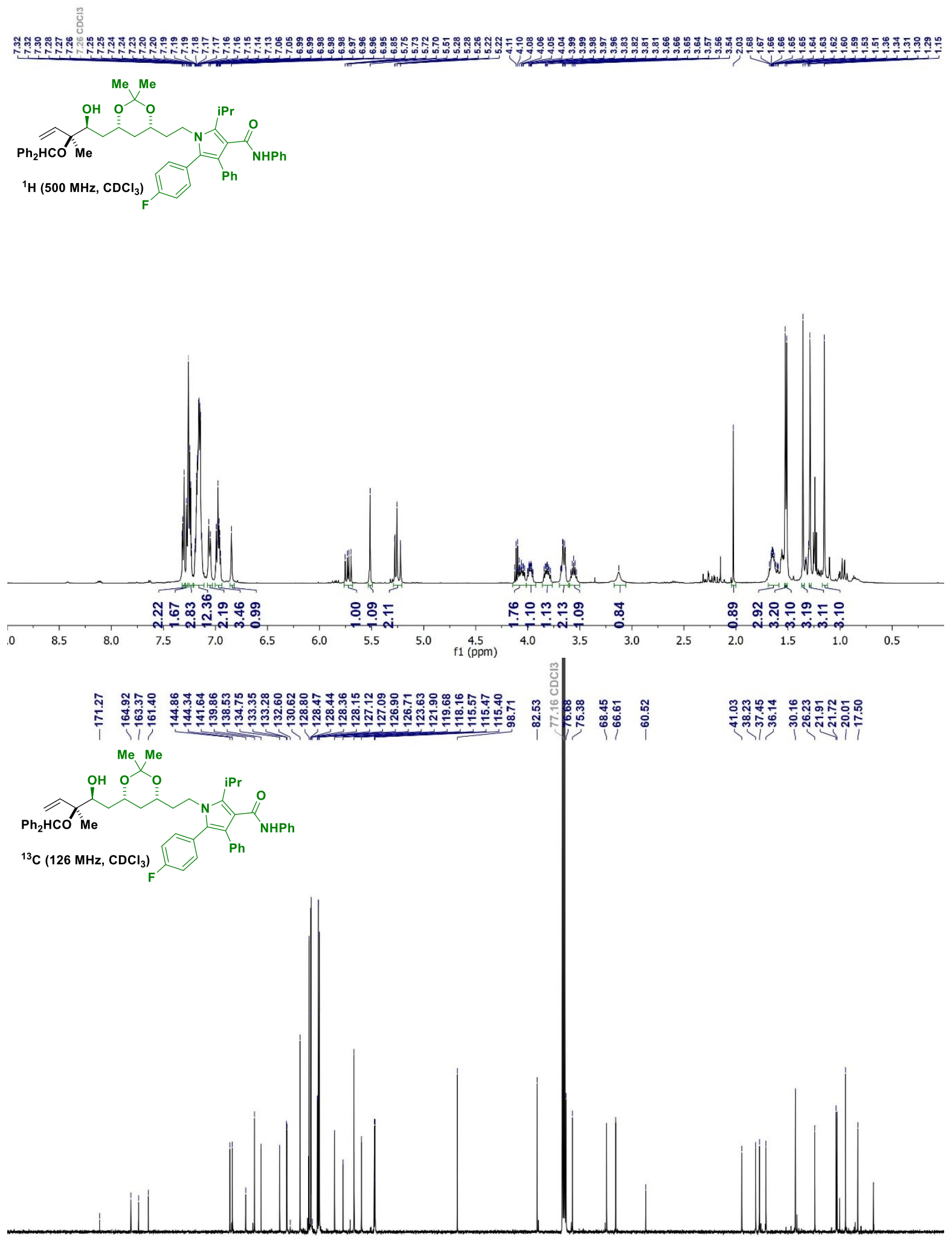

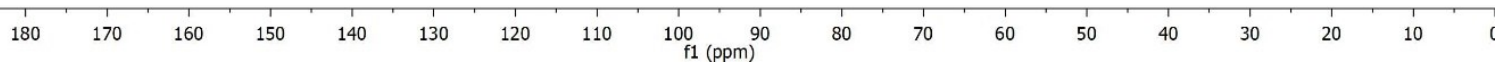




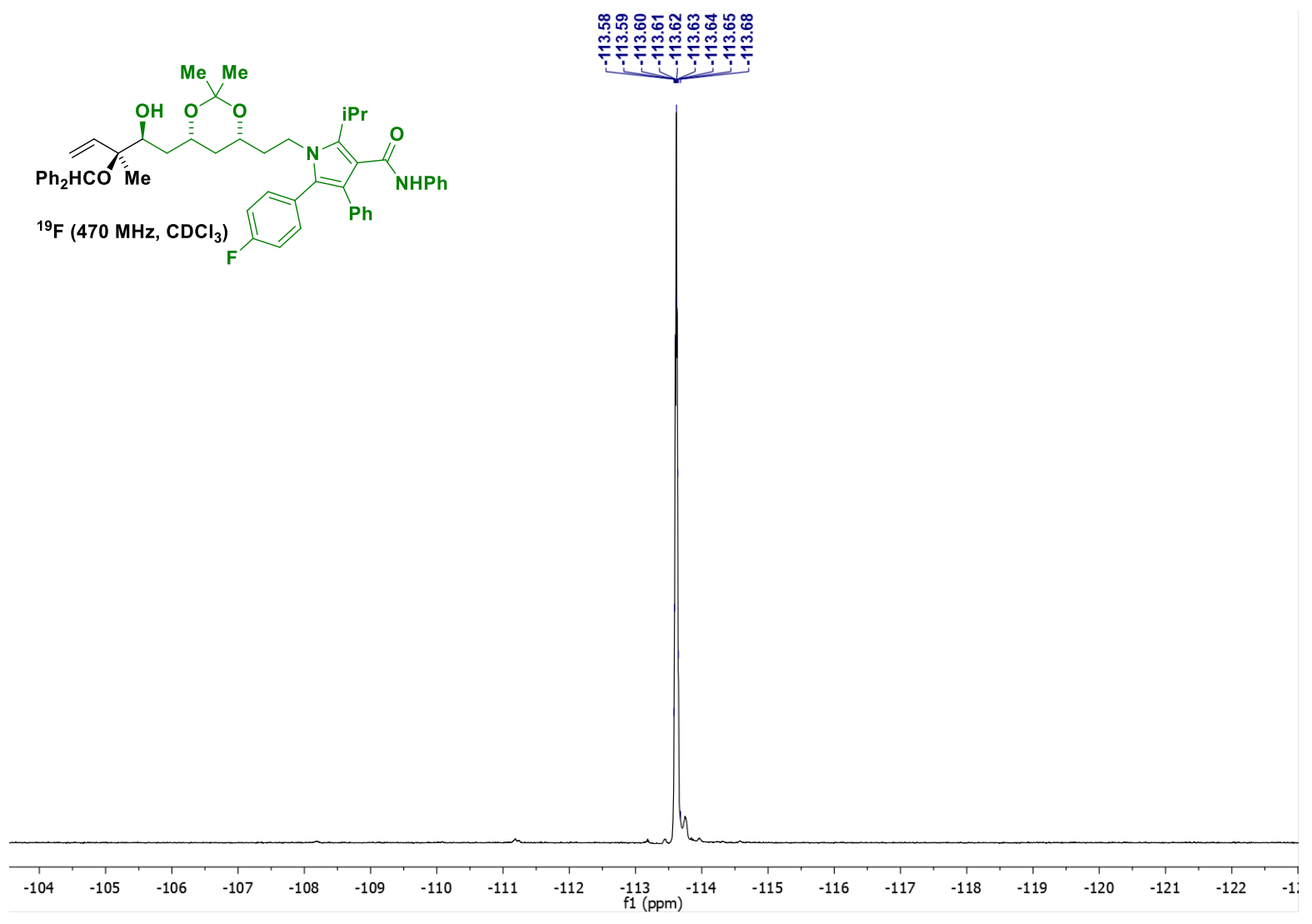


1-(2-((4R,6S)-6-((2R,3R)-3-(benzhydryloxy)-2-hydroxy-3-methylpent-4-en-1-yl)-2,2-dimethyl1,3-dioxan-4-yl)ethyl)-5-(4-fluorophenyl)-2-isopropyl-N,4-diphenyl-1H-pyrrole-3-carboxamide (iso-4jj)

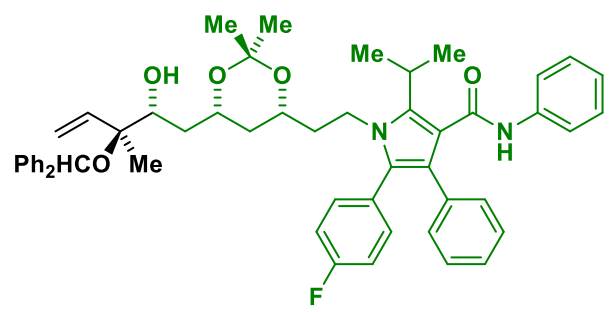

Aldehyde oxidation level: Aldehyde $3 \mathbf{j j}(116.5 \mathrm{mg}, 0.2 \mathrm{mmol})$ was subjected to standard reaction conditions, (S)-BINAP was used (10 mol\% catalyst loading, $75{ }^{\circ} \mathrm{C}, 48 \mathrm{~h}$ ). Upon flash column chromatography ( $\mathrm{SiO}_{2}, 30: 70$ EtOAc:hexanes), the title compound iso-4jj was obtained as a yellow oil in $65 \%$ yield $(106.7 \mathrm{mg}, 0.13 \mathrm{mmol}, 11: 1 \mathrm{dr}$ ).

$\operatorname{TLC}\left(\mathrm{SiO}_{2}\right) \mathrm{R}_{\mathrm{f}}=0.5$ (40:60 EtOAc:hexanes)

${ }^{1} \mathrm{H}$ NMR $\left(500 \mathrm{MHz}, \mathrm{CDCl}_{3}\right) \delta: 7.33(\mathrm{~d}, \mathrm{~J}=2.0 \mathrm{~Hz}, 1 \mathrm{H}), 7.31(\mathrm{~d}, \mathrm{~J}=1.5 \mathrm{~Hz}, 1 \mathrm{H}), 7.30(\mathrm{~s}, 1 \mathrm{H}), 7.28(\mathrm{~d}$, $\mathrm{J}=1.8 \mathrm{~Hz}, 2 \mathrm{H}), 7.27(\mathrm{~d}, \mathrm{~J}=2.3 \mathrm{~Hz}, 2 \mathrm{H}), 7.22-7.15(\mathrm{~m}, 12 \mathrm{H}), 7.07(\mathrm{~d}, \mathrm{~J}=8.0 \mathrm{~Hz}, 2 \mathrm{H}), 7.01-6.97$ $(\mathrm{m}, 3 \mathrm{H}), 6.86(\mathrm{~s}, 1 \mathrm{H}), 5.74(\mathrm{dd}, \mathrm{J}=17.6,11.0 \mathrm{~Hz}, 1 \mathrm{H}), 5.53(\mathrm{~s}, 1 \mathrm{H}), 5.33-5.21(\mathrm{~m}, 2 \mathrm{H}), 4.15-3.97$ $(\mathrm{m}, 3 \mathrm{H}), 3.87-3.79(\mathrm{~m}, 2 \mathrm{H}), 3.67(\mathrm{tq}, \mathrm{J}=7.3,2.5 \mathrm{~Hz}, 1 \mathrm{H}), 3.57$ (hept, J = $7.4 \mathrm{~Hz}, 1 \mathrm{H}), 2.78(\mathrm{~s}, 1 \mathrm{H})$, $2.05(\mathrm{~s}, 1 \mathrm{H}), 1.75-1.60(\mathrm{~m}, 3 \mathrm{H}), 1.54(\mathrm{~s}, 3 \mathrm{H}), 1.53(\mathrm{~s}, 3 \mathrm{H}), 1.35(\mathrm{~s}, 3 \mathrm{H}), 1.30(\mathrm{~s}, 3 \mathrm{H}), 1.12(\mathrm{~s}, 3 \mathrm{H})$.

${ }^{13}$ C NMR $\left(125 \mathrm{MHz}, \mathrm{CDCl}_{3}\right) \delta: 171.3,164.16(\mathrm{~d}, J=197.2 \mathrm{~Hz}), 161.4,144.8,144.3,141.7,140.2$, 138.6, 134.8, 133.33 (d, J = 8.1 Hz), 130.6, 128.8, 128.5, 128.4, 127.7, 127.2, 127.2, 127.0, 126.7, 123.6, 121.9, 119.7, 118.4, 115.46 (d, $J=21.4 \mathrm{~Hz}$ ), 115.4, 98.7, 82.8, 76.6, 73.2, 66.8, 66.3, 60.5, $41.1,38.3,37.8,37.0,30.2,26.2,21.9,21.7,19.9,19.4,16.3$.

${ }^{19}$ F NMR $\left(470 \mathrm{MHz}, \mathrm{CDCl}_{3}\right) \delta:-113.8(\mathrm{tt}, \mathrm{J}=8.8,5.4 \mathrm{~Hz})$.

HRMS $(\mathrm{Na}+, m / z)$ for $\mathrm{C}_{53} \mathrm{H}_{57} \mathrm{FN}_{2} \mathrm{O}_{5}$ : calcd. $=843.4144 ;$ found $=843.4147$.

FTIR (neat): 3646, 2246, 1731, 1661, 1525, 1435, 1241, 1156, 1093, 906, $727 \mathrm{~cm}^{-1}$

$[\alpha]_{D}^{24}=-28.0^{\circ}\left(\mathrm{c}=0.2, \mathrm{CHCl}_{3}\right)$ 


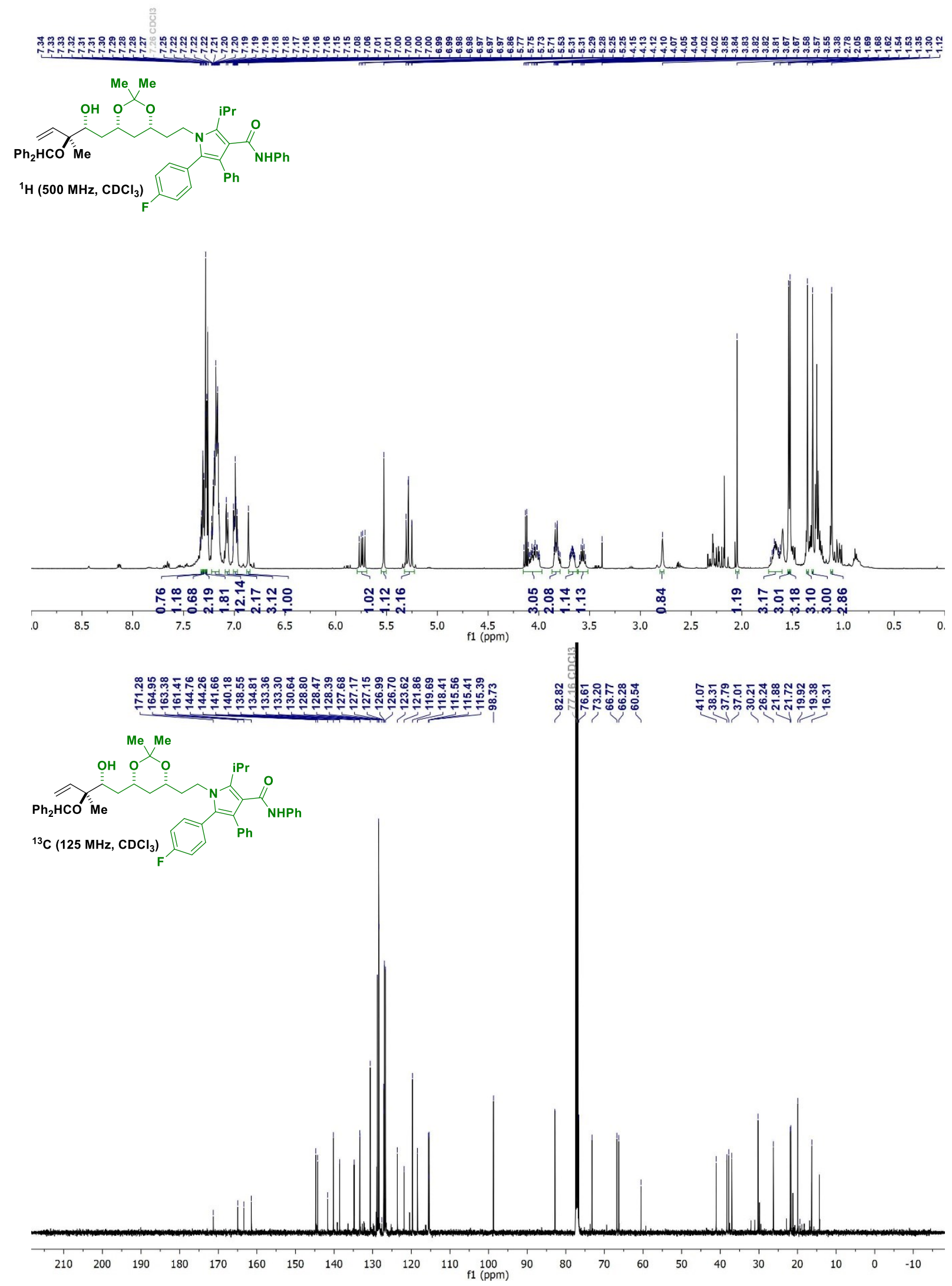



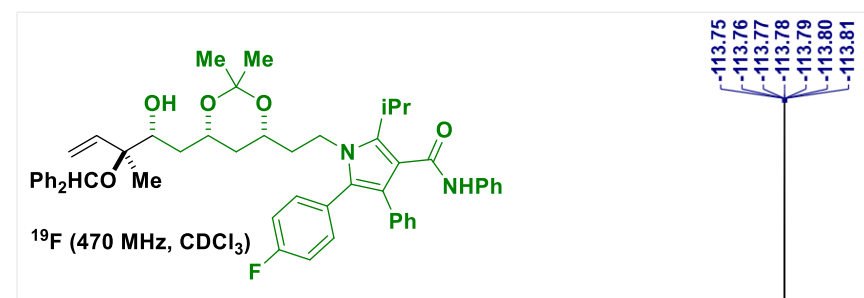

造

$\mathrm{F}$

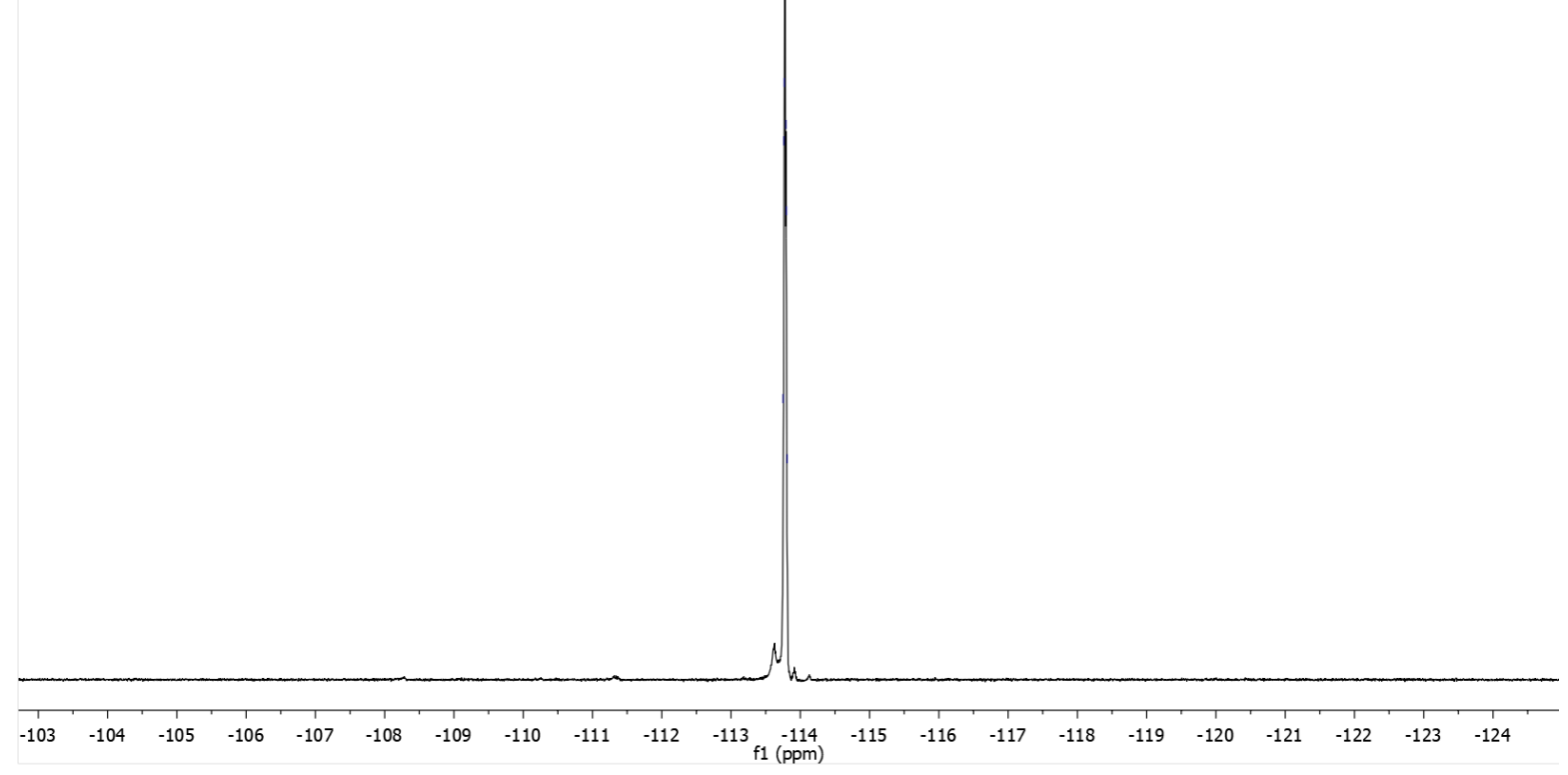




\section{Total Syntheses of (-)-Citreodiol}

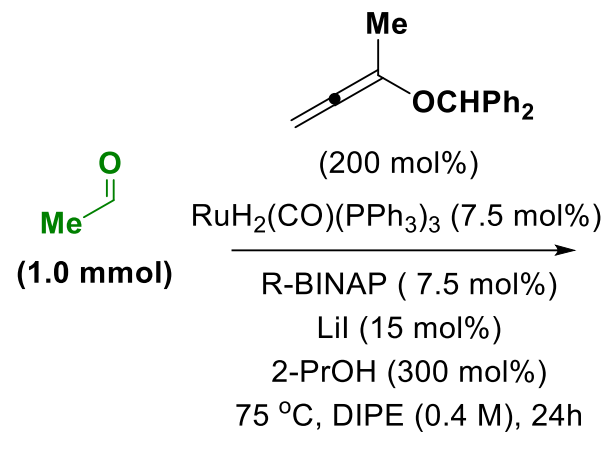

1: Hoveyda-Grubbs II (7 mol\%) Acrolein (1000 mol\%) DCM/THF $(4: 1,0.04 \mathrm{M})$

$2:$

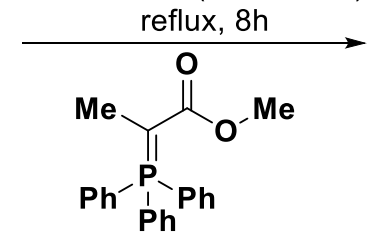

Benzene $(0.04 \mathrm{M})$ reflux, $4 \mathrm{~h}$

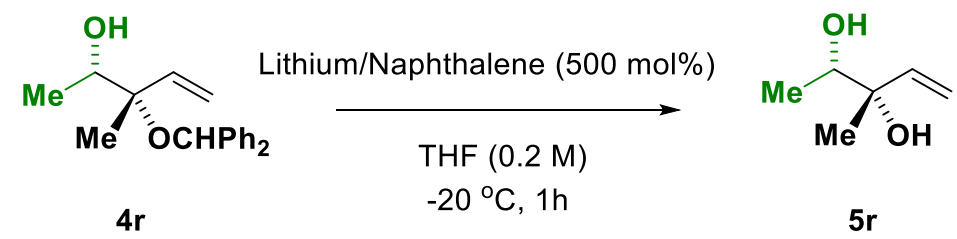

$77 \%$ Yield $15: 1 \mathrm{dr}, 87 \%$ ee

$78 \%$ Yield $>20: 1 \mathrm{dr}$

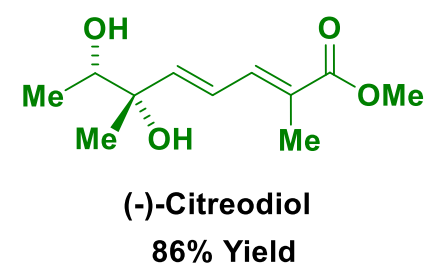




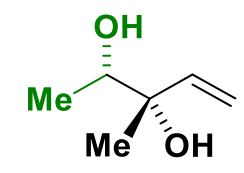

To a solution of $4 \mathrm{r}(206.0 \mathrm{mg}, 0.729 \mathrm{mmol})$ in THF $(3.6 \mathrm{~mL}, 0.2 \mathrm{M})$ at $-20^{\circ} \mathrm{C}$ was added freshly prepared lithium naphthalenide $(3.65 \mathrm{~mL}, 3.65 \mathrm{mmol}, 500 \mathrm{~mol} \%)$ in THF. ${ }^{5}$ After 1 hour at $-20{ }^{\circ} \mathrm{C}$, saturated $\mathrm{NH}_{4} \mathrm{Cl}$ (aq) $(5 \mathrm{~mL}$ ) was added to the reaction mixture. The reaction mixture was trnasferred to a separatory funnel and the organic layer was extracted with ether. The combined organic extracts were dried $\left(\mathrm{Na}_{2} \mathrm{SO}_{4}\right)$, filtered and concentrated in vacuo and the residue was subjected to column chromatography $\left(\mathrm{SiO}_{2}: 30: 70 \mathrm{EtOAc} /\right.$ hexanes$)$ to afford the title compound (66.0 mg, $0.568 \mathrm{mmol},>20: 1 \mathrm{dr}$ ) in $78 \%$ yield as a colorless oil.

$\operatorname{TLC}\left(\mathrm{SiO}_{2}\right) \mathrm{R}_{\mathrm{f}}=0.2$ (30:70 EtOAc:hexanes)

${ }^{1} \mathrm{H}$ NMR $\left(500 \mathrm{MHz}, \mathrm{CDCl}_{3}\right) \delta: 5.91$ (dd, $\left.J=17.4,10.8 \mathrm{~Hz}, 1 \mathrm{H}\right), 5.36$ (dd, $\left.J=17.4,1.3 \mathrm{~Hz}, 1 \mathrm{H}\right), 5.19$ (dd, $J=10.8,1.3 \mathrm{~Hz}, 1 \mathrm{H}), 3.66(\mathrm{q}, J=6.4 \mathrm{~Hz}, 1 \mathrm{H}), 1.97(\mathrm{~s}, 2 \mathrm{H}), 1.23(\mathrm{~s}, 3 \mathrm{H}), 1.16(\mathrm{~d}, J=6.5 \mathrm{~Hz}, 3 \mathrm{H}$ ).

${ }^{13} \mathrm{C}$ NMR $\left(125 \mathrm{MHz}, \mathrm{CDCl}_{3}\right) \delta: 142.9,114.5,75.7,73.0,21.6,16.7$.

FTIR (neat): 3350, 2984, 1373, 1275, 1214, 1093, $997 \mathrm{~cm}^{-1}$

HRMS $(\mathrm{Na}+, \mathrm{m} / z)$ for $\mathrm{C}_{6} \mathrm{H}_{12} \mathrm{O}_{2}$ : calcd. $=139.0730$, found $=139.0728$.

$[\alpha]_{D}^{24}=-1.2^{\circ}\left(\mathrm{c}=0.2, \mathrm{CHCl}_{3}\right)$ 


$$
\text { Me }
$$

${ }^{1} \mathrm{H}\left(500 \mathrm{MHz}, \mathrm{CDCl}_{3}\right)$
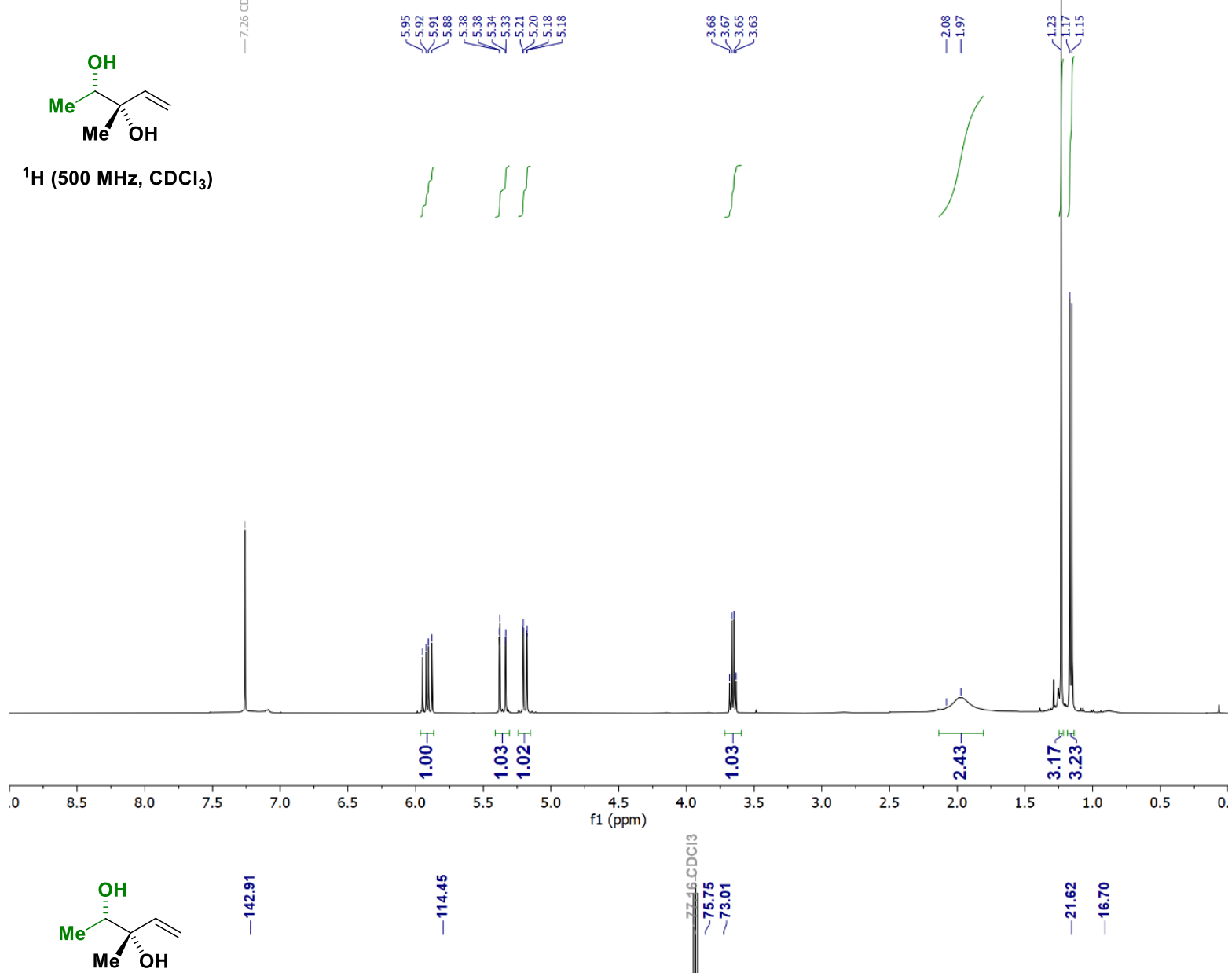

$\stackrel{\frac{?}{+}}{\frac{1}{7}}$

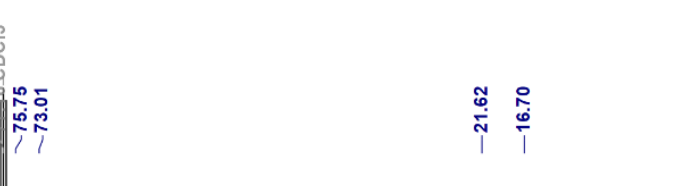

ํํำ
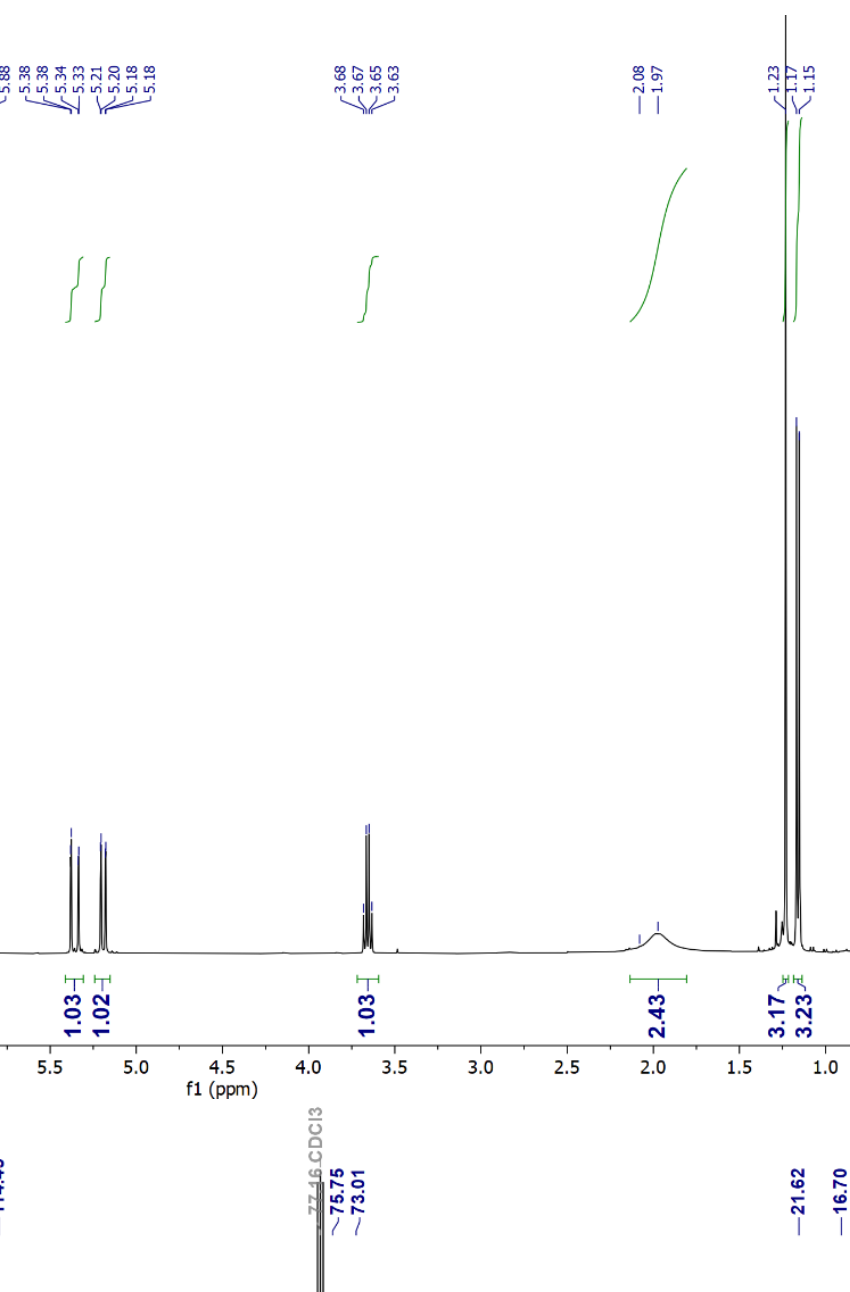

${ }^{13} \mathrm{C}\left(125 \mathrm{MHz}, \mathrm{CDCl}_{3}\right)$

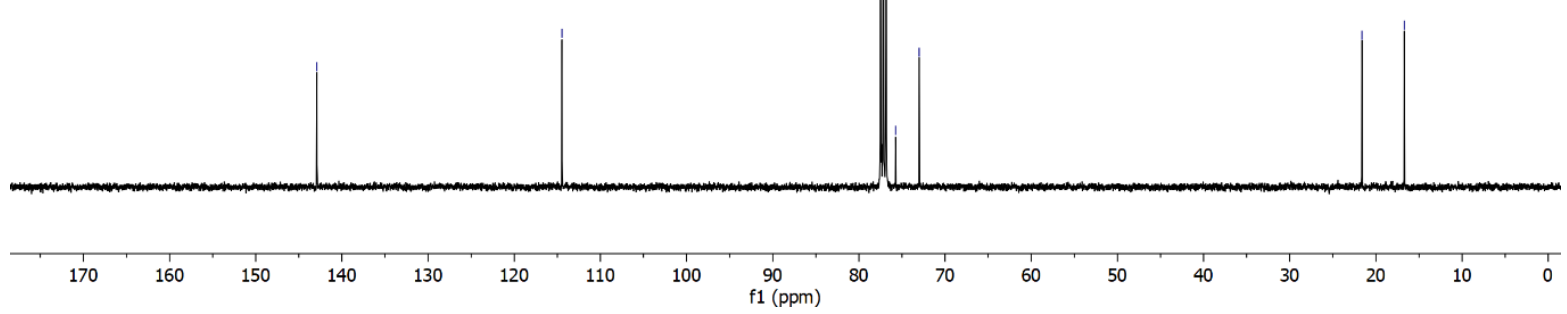


methyl (2E,4E,6S,7S)-6,7-dihydroxy-2,6-dimethylocta-2,4-dienoate ((-)-Citreodiol)<smiles>COC(=O)/C(C)=C/C=C/[C@@](C)(O)C(C)O</smiles>

(-)-Citreodiol

To a solution of $5 \mathbf{r}(40.7 \mathrm{mg}, 0.35 \mathrm{mmol})$ in DCM/THF (4:1) $(10 \mathrm{~mL}, 0.04 \mathrm{M})$ was added HoveydaGrubbs II catalyst $(15.3 \mathrm{mg}, 0.0245 \mathrm{mmol}, 7 \mathrm{~mol} \%)$ and freshly distilled acrolein $(0.23 \mathrm{~mL}, 3.5$ mmol, 1000 mol\%). ${ }^{6,7}$ The reaction mixture was heated to reflux fand was allowed to stir for 8 hours. The reaction mixture was allowed to cool to room temperature, at which point the solvent was removed in vacuo. The crude residue was dissolved in dry benzene $(8.7 \mathrm{~mL}, 0.04 \mathrm{M})$ and methyl-2-(triphenylphosphoranylidene)-propanoate $(245 \mathrm{mg}, 0.7 \mathrm{mmol}, 200 \mathrm{~mol} \%$ ) was added. The reactio mixture was heated to reflux and was allowed to stir for 4 hours. The solvent was removed in vacuo and the residue was subjected to silica gel column chromatography (60:40 $\mathrm{Et}_{2} \mathrm{O} /$ hexanes) to obtain (-)-citreodiol ( $64.5 \mathrm{mg}, 0.3 \mathrm{mmol}$ ) in $86 \%$ yield as a light yellow oil.

$\operatorname{TLC}\left(\mathrm{SiO}_{2}\right) \mathrm{R}_{\mathrm{f}}=0.2\left(60: 40 \mathrm{Et}_{2} \mathrm{O}\right.$ :hexanes)

${ }^{1} \mathrm{H}$ NMR $\left(500 \mathrm{MHz}, \mathrm{CDCl}_{3}\right) \delta: 7.18(\mathrm{~d}, J=11.5 \mathrm{~Hz}, 1 \mathrm{H}), 6.67(\mathrm{dd}, J=15.2,11.4 \mathrm{~Hz}, 1 \mathrm{H}), 6.09(\mathrm{~d}, J=$ $15.2 \mathrm{~Hz}, 1 \mathrm{H}), 3.75(\mathrm{~s}, 3 \mathrm{H}), 3.69(\mathrm{q}, J=6.4 \mathrm{~Hz}, 1 \mathrm{H}), 2.27(\mathrm{~s}, 1 \mathrm{H}), 2.17-2.04(\mathrm{~s}, 1 \mathrm{H}), 1.96(\mathrm{~d}, J=1.4$ $\mathrm{Hz}, 3 \mathrm{H}) 1.27(\mathrm{~s}, 3 \mathrm{H}), 1.17(\mathrm{~d}, J=6.4 \mathrm{~Hz}, 3 \mathrm{H})$.

${ }^{13} \mathrm{C} \mathrm{NMR}\left(125 \mathrm{MHz}, \mathrm{CDCl}_{3}\right) \delta: 169.0,145.4,137.6,127.6,124.9,75.8,73.2,52.0,22.1,17.1,12.9$.

FTIR (neat): 3350, 3018, 1698, 1275, 1214, 1106, $977 \mathrm{~cm}^{-1}$

HRMS $(\mathrm{Na}+, \mathrm{m} / z)$ for $\mathrm{C}_{11} \mathrm{H}_{18} \mathrm{O}_{4}$ : calcd. = 237.1097; found $=237.1100$.

$[\alpha]_{D}^{24}=-4.3^{\circ}\left(\mathrm{c}=0.23, \mathrm{CHCl}_{3}\right)$. 

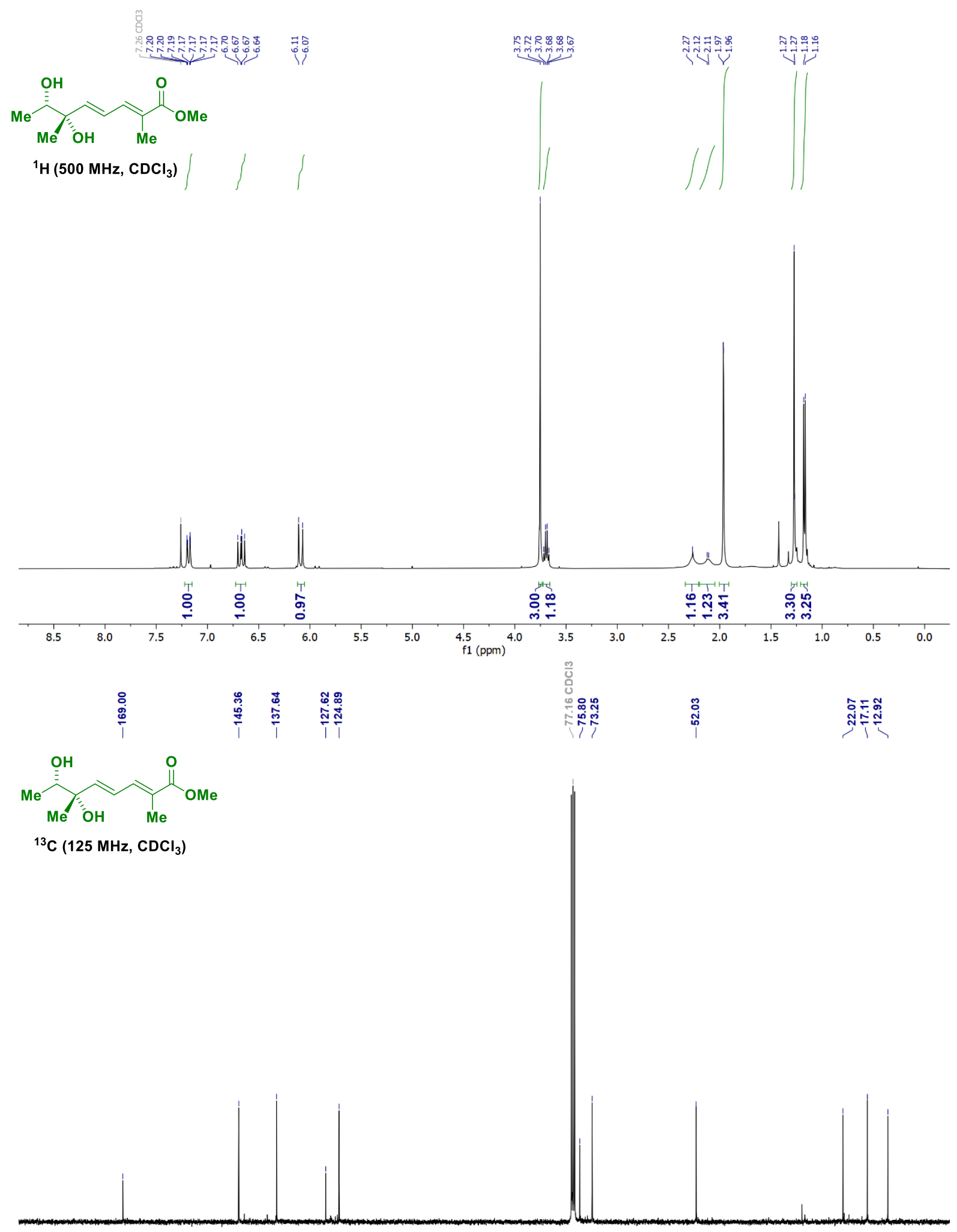

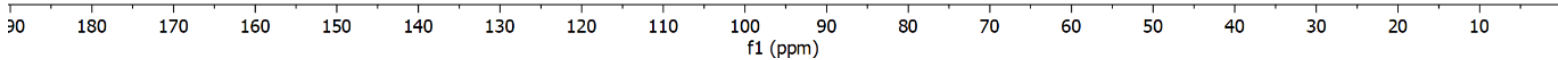




\section{Isotopic Labeling Studies}
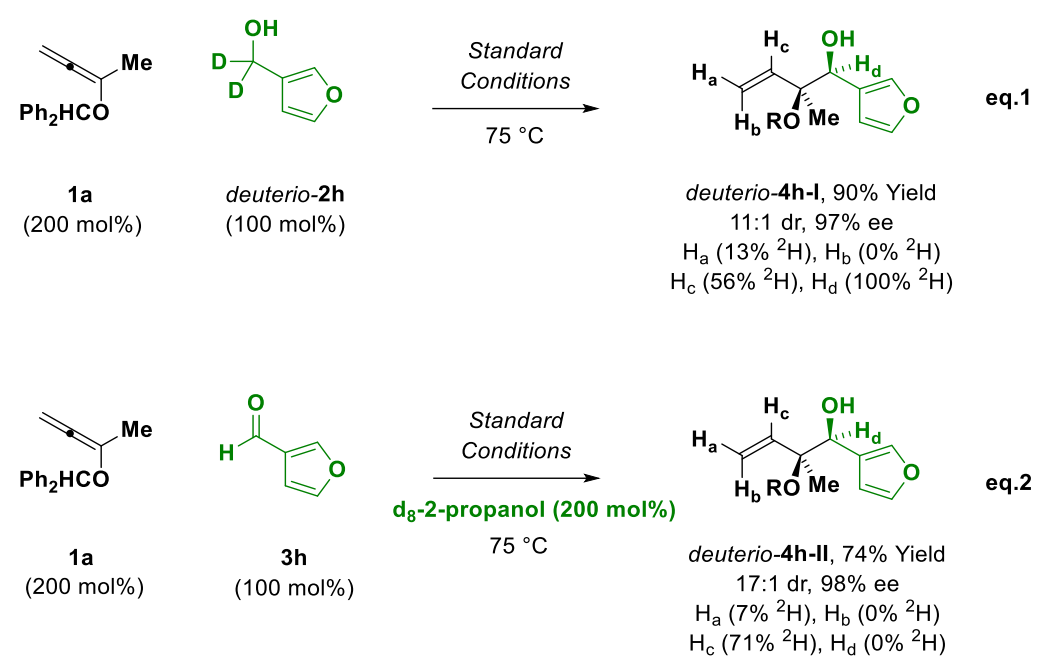

deuterio-2h:

${ }^{1} \mathrm{H}$ NMR $\left(400 \mathrm{MHz}, \mathrm{CDCl}_{3}\right) \delta: 7.42(\mathrm{t}, J=1.2 \mathrm{~Hz}, 1 \mathrm{H}), 7.40(\mathrm{t}, J=1.7 \mathrm{~Hz}, 1 \mathrm{H}), 6.44(\mathrm{dd}, J=2.0,0.9$ $\mathrm{Hz}, 1 \mathrm{H}), 1.64(\mathrm{~d}, J=4.0 \mathrm{~Hz}, 1 \mathrm{H})$.

${ }^{13} \mathrm{C}$ NMR $\left(100 \mathrm{MHz}, \mathrm{CDCl}_{3}\right) \delta: 143.6,140.1,125.2,109.9,56.2(p, J=22.1 \mathrm{~Hz})$.

Eq1: Alcohol deuterio- $2 \mathrm{~h}$ (100 mol\%, $0.2 \mathrm{mmol}, 20.0 \mathrm{mg}$ ) was subjected to standard reaction conditions $\left(75^{\circ} \mathrm{C}, 24 \mathrm{~h}\right)$. Upon flash column chromatography $\left(\mathrm{SiO}_{2}, 5: 95\right.$ EtOAc:hexanes), the title compound deuterio-4h-I ( $60.2 \mathrm{mg}, 0.18 \mathrm{mmol}, 11: 1 \mathrm{dr}, 97 \%$ ee) was obtained as a light yellow oil in $90 \%$ yield.

${ }^{1} \mathbf{H}$ NMR $\left(600 \mathrm{MHz}, \mathrm{CDCl}_{3}\right) \delta 7.38-7.34(\mathrm{~m}, 4 \mathrm{H}), 7.34-7.29(\mathrm{~m}, 6 \mathrm{H}), 7.26-7.22(\mathrm{~m}, 2 \mathrm{H}), 6.41$ $(\mathrm{dd}, J=1.9,0.8 \mathrm{~Hz}, 1 \mathrm{H}), 5.89-5.83(\mathrm{~m}, 0.44 \mathrm{H}), 5.59(\mathrm{~s}, 1 \mathrm{H}), 5.32-5.29(\mathrm{~m}, 0.87 \mathrm{H}), 5.22-5.18$ $(\mathrm{m}, 1 \mathrm{H}), 3.13(\mathrm{~s}, 1 \mathrm{H}), 1.13(\mathrm{~s}, 3 \mathrm{H})$.

${ }^{2} \mathrm{H}$ NMR (92 MHz, $\left.\mathrm{CHCl}_{3}\right) \delta 5.91$ (s, 0.57D), 5.32 (s, 0.14D), 4.73 (s, 1D).

Eq2: Aldehyde $3 \mathrm{~h}$ (100 mol\%, $0.2 \mathrm{mmol}, 19.2 \mathrm{mg}$ ) was subjected to standard reaction conditions ( $75^{\circ} \mathrm{C}, 24 \mathrm{~h}$ ) using $\mathbf{d}_{\mathbf{8}}$-2-propanol as a reductant (200 mol\%). Upon flash column chromatography $\left(\mathrm{SiO}_{2}, 5: 95\right.$ EtOAc:hexanes), the title compound deuterio-4h-II $(49.8 \mathrm{mg}, 0.148 \mathrm{mmol}, 17: 1 \mathrm{dr}$, $98 \%$ ee) was obtained as a light yellow oil in $74 \%$ yield.

${ }^{1} \mathrm{H}$ NMR $\left(600 \mathrm{MHz}, \mathrm{CDCl}_{3}\right) \delta 7.38-7.33(\mathrm{~m}, 4 \mathrm{H}), 7.33-7.28(\mathrm{~m}, 6 \mathrm{H}), 7.25-7.21(\mathrm{~m}, 2 \mathrm{H}), 6.41-$ $6.38(\mathrm{~m}, 1 \mathrm{H}), 5.88-5.81(\mathrm{~m}, 0.29 \mathrm{H}), 5.58(\mathrm{~s}, 1 \mathrm{H}), 5.31-5.29(\mathrm{~m}, 0.93 \mathrm{H}), 5.21-5.17(\mathrm{~m}, 1 \mathrm{H}), 4.71$ $(\mathrm{d}, J=3.0 \mathrm{~Hz}, 1 \mathrm{H}), 3.12(\mathrm{~d}, J=3.2 \mathrm{~Hz}, 1 \mathrm{H}), 1.11(\mathrm{~s}, 3 \mathrm{H})$.

${ }^{2} \mathrm{H}$ NMR (92 MHz, $\mathrm{CHCl}_{3}$ ) $\delta 5.92$ (s, 0.71D), 5.33 (s, 0.06D).

HPLC: (Two connected Chiralcel ADH columns, Hexane:2-PrOH = 96:4, 0.5 mL/min, $210 \mathrm{~nm}$ ) 


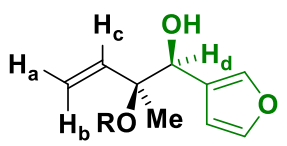

deuterio-4h-I

${ }^{1} \mathrm{H}$ NMR $\left(600 \mathrm{MHz}, \mathrm{CDCl}_{3}\right)$
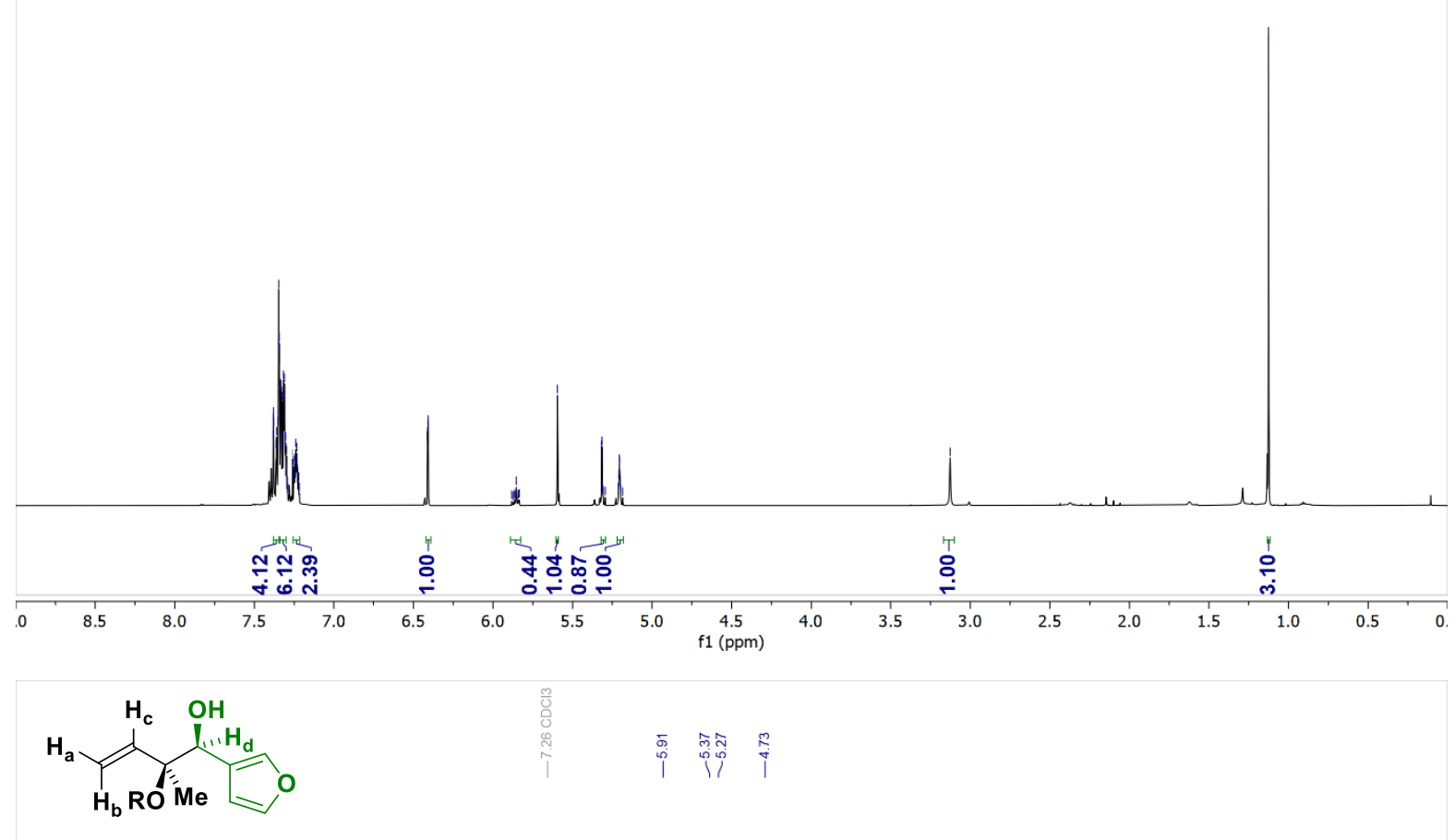

i.

deuterio-4h-I

${ }^{2} \mathrm{H} \mathrm{NMR}\left(92 \mathrm{MHz}, \mathrm{CHCl}_{3}\right)$

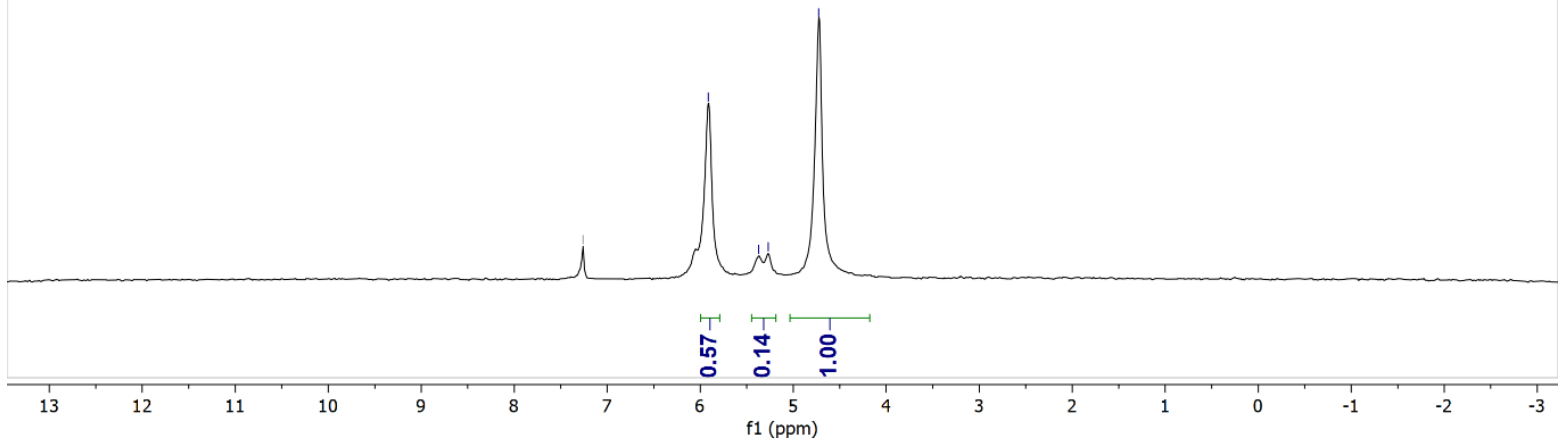



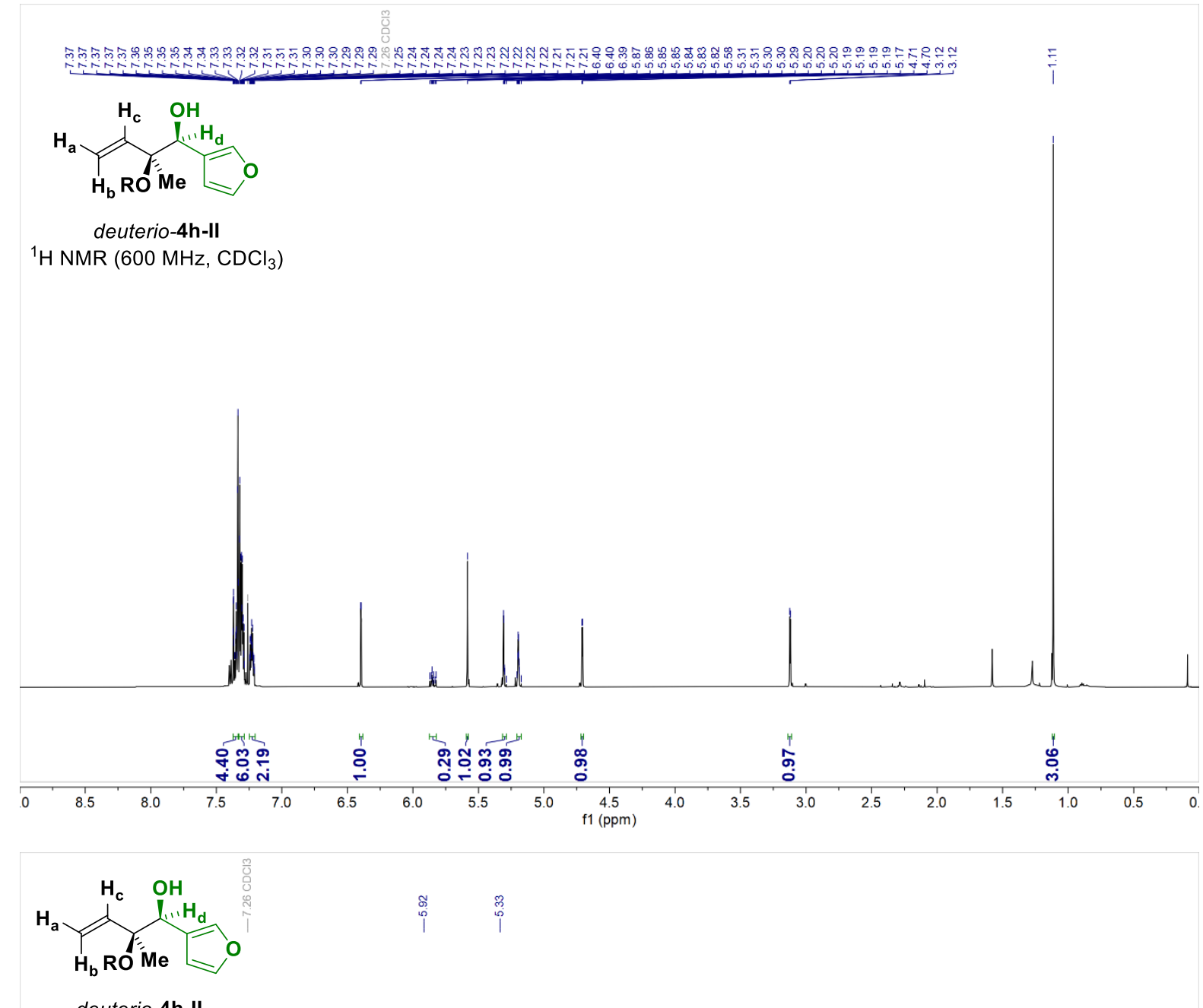

${ }^{2} \mathrm{H}$ NMR (92 $\mathrm{MHz}, \mathrm{CHCl}_{3}$ )

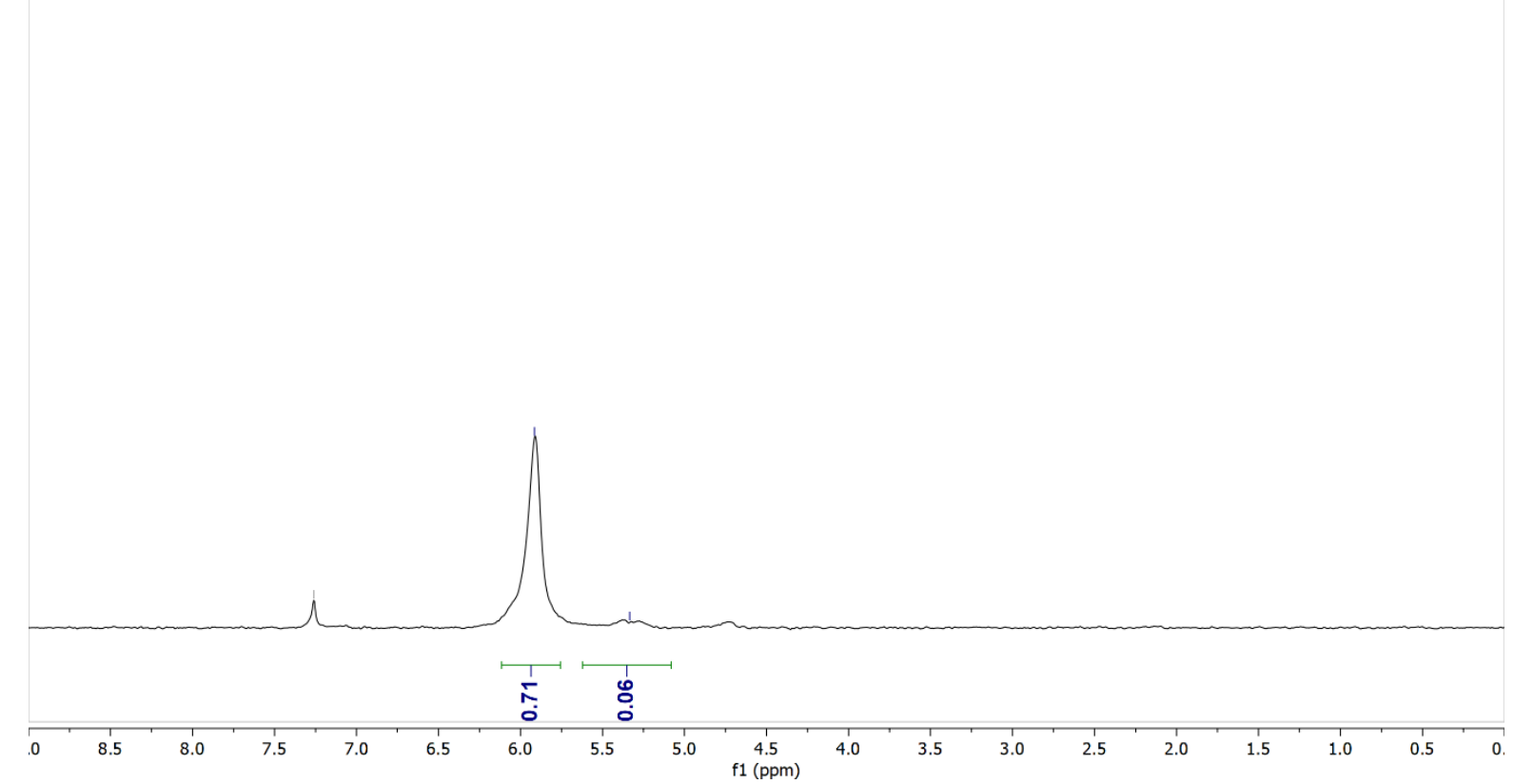



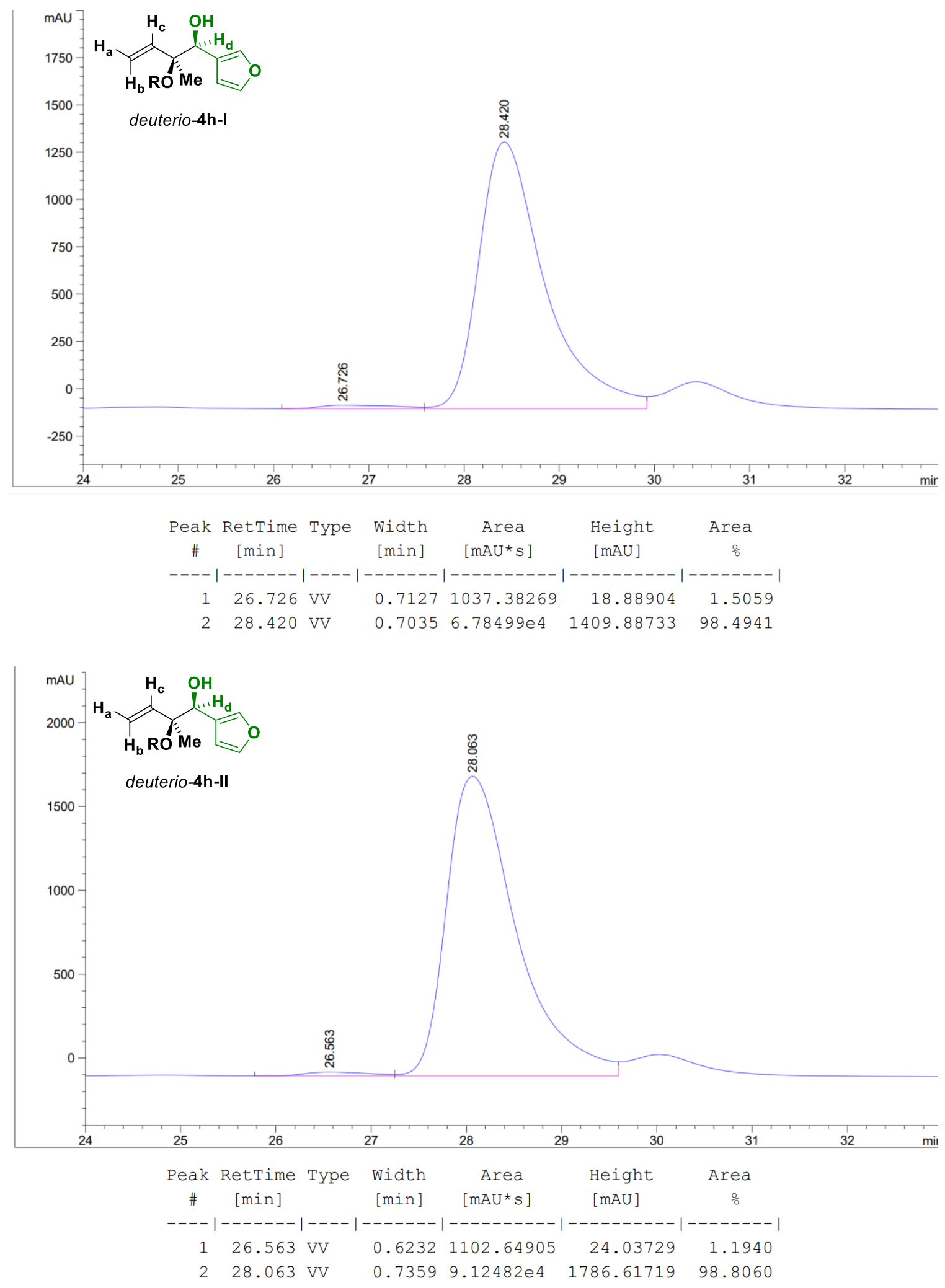
(1S,2S)-2-(benzyloxy)-1-(4-bromophenyl)-2-methylbut-3-en-1-ol (s4a)

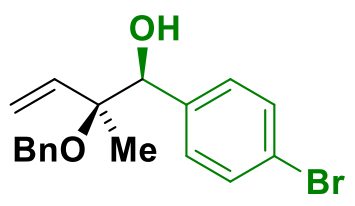

Aldehyde oxidation level: Aldehyde $3 a(37.0 \mathrm{mg}, 0.2 \mathrm{mmol})$ was subjected to standard reaction conditions [allene $\mathbf{1 b}(64.0 \mathrm{mg}, 200 \mathrm{~mol} \%), 75{ }^{\circ} \mathrm{C}, 24 \mathrm{~h}$ ]. Upon flash column chromatography $\left(\mathrm{SiO}_{2}, 5: 95\right.$ EtOAc:hexanes), the title compound $\mathbf{s} 4 \mathrm{a}$ was obtained as a colorless oil in $73 \%$ yield (50.7 mg, $0.146 \mathrm{mmol}, 15: 1 \mathrm{dr}, 77 \%$ ee).

$\operatorname{TLC}\left(\mathrm{SiO}_{2}\right) \mathrm{R}_{\mathrm{f}}=0.3$ (10:90 EtOAc:hexanes)

${ }^{1} \mathrm{H}$ NMR $\left(500 \mathrm{MHz}, \mathrm{CDCl}_{3}\right) \delta: 7.46-7.40(\mathrm{~m}, 2 \mathrm{H}), 7.40-7.28(\mathrm{~m}, 5 \mathrm{H}), 7.28-7.22(\mathrm{~m}, 2 \mathrm{H}), 5.81$ (dd, $J=17.7,10.9 \mathrm{~Hz}, 1 \mathrm{H}), 5.36(\mathrm{dd}, J=10.9,1.1 \mathrm{~Hz}, 1 \mathrm{H}), 5.19(\mathrm{dd}, J=17.7,1.2 \mathrm{~Hz}, 1 \mathrm{H}), 4.64(\mathrm{~s}$, $1 \mathrm{H}), 4.51-4.37(\mathrm{~m}, 2 \mathrm{H}), 3.26(\mathrm{~s}, 1 \mathrm{H}), 1.20(\mathrm{~s}, 3 \mathrm{H})$.

${ }^{13} \mathrm{C} \mathrm{NMR}\left(125 \mathrm{MHz}, \mathrm{CDCl}_{3}\right)$ 8: 139.3, 138.9, 138.2, 130.7, 129.7, 128.6, 127.7, 127.6, 121.6, 118.7, $81.7,79.5,65.1,14.9$.

HRMS $(\mathrm{Na}+, \mathrm{m} / z)$ for $\mathrm{C}_{18} \mathrm{H}_{19} \mathrm{BrO}_{2}$ : calcd. $=369.0461 ;$ found $=369.0468$.

FTIR (neat): 3350, 2989, 1264, 1010, 733, $702 \mathrm{~cm}^{-1}$

HPLC: (Two connected Chiralcel AD-H columns, Hexane:2-PrOH = 98:02, $1.0 \mathrm{~mL} / \mathrm{min}, 210 \mathrm{~nm}$ ) $[\alpha]_{D}^{24}=+3.8^{\circ}\left(\mathrm{c}=0.4, \mathrm{CHCl}_{3}\right)$. 


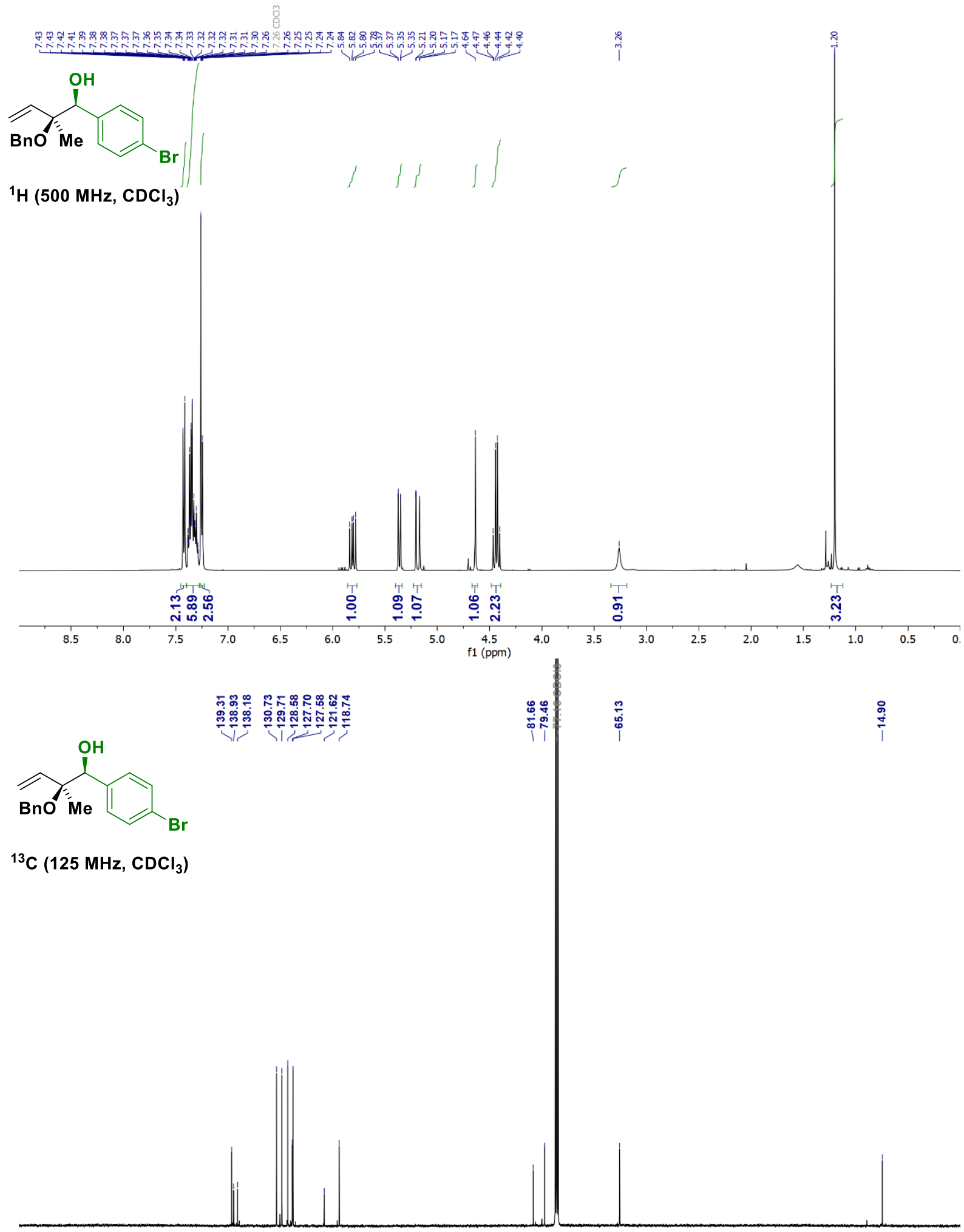

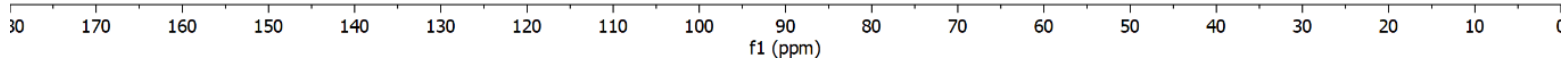




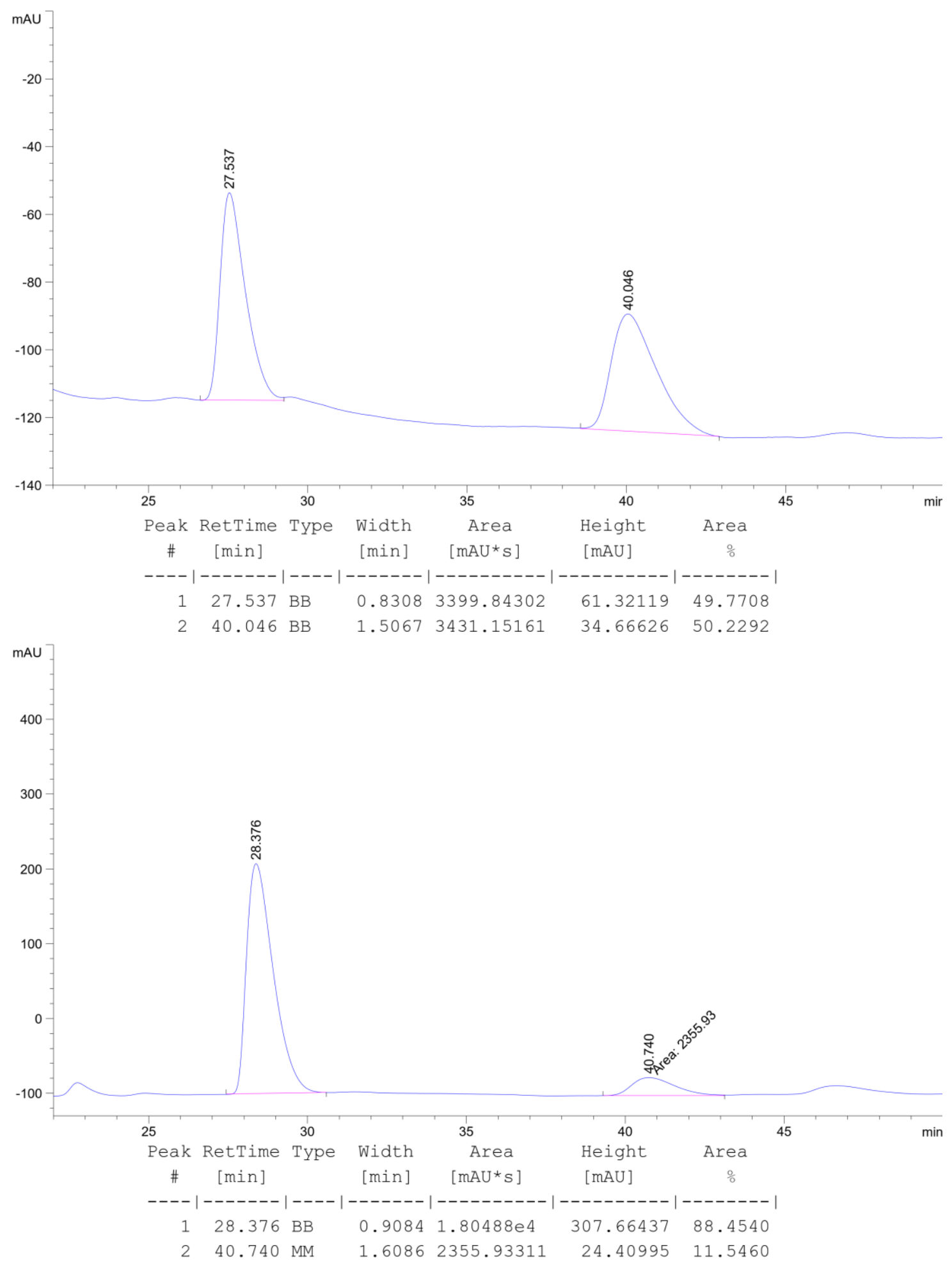




\section{Single Crystal Diffraction Data for Coupling Product 4b}

Empirical formula

Formula weight

Temperature

Wavelength

Crystal system

Space group

Unit cell dimensions

Volume

Z

Density (calculated)

Absorption coefficient

$\mathrm{F}(000)$

Crystal size

Theta range for data collection

Index ranges

Reflections collected

Independent reflections

Completeness to theta $=67.684^{\circ}$

Absorption correction

Max. and min. transmission

Refinement method

Data / restraints / parameters

Goodness-of-fit on $\mathrm{F}^{2}$

Final R indices [I $>2 \operatorname{sigma}(\mathrm{I})]$

$\mathrm{R}$ indices (all data)

Absolute structure parameter

Extinction coefficient

Largest diff. peak and hole
C30 H35 B O4

470.39

100.01(13) K

$1.54184 \AA$

orthorhombic

P 212121

$\mathrm{a}=9.0877(2) \AA$

$\alpha=90^{\circ}$.

$\mathrm{b}=13.8537(3) \AA$

$\beta=90^{\circ}$.

$\mathrm{c}=20.5694(5) \AA$

$\gamma=90^{\circ}$.

2589.65(10) $\AA^{3}$

4

$1.206 \mathrm{Mg} / \mathrm{m}^{3}$

$0.614 \mathrm{~mm}^{-1}$

1008

$0.39 \times 0.284 \times 0.25 \mathrm{~mm}^{3}$

3.847 to $73.625^{\circ}$.

$-11<=\mathrm{h}<=11,-16<=\mathrm{k}<=16,-25<=\mathrm{k}<=18$

24026

$5137[\mathrm{R}(\mathrm{int})=0.0489]$

$100.0 \%$

Gaussian and multi-scan

1.000 and 0.382

Full-matrix least-squares on $\mathrm{F}^{2}$

5137 / 0 / 330

1.049

$\mathrm{R} 1=0.0348, \mathrm{wR} 2=0.0917$

$\mathrm{R} 1=0.0356, \mathrm{wR} 2=0.0927$

$-0.06(9)$

$\mathrm{n} / \mathrm{a}$

0.251 and -0.201 e. $\AA^{-3}$ 
Figure 1. View of $\mathbf{4 b}$ showing the atom labeling scheme. Displacement ellipsoids are scaled to the $50 \%$ probability level.

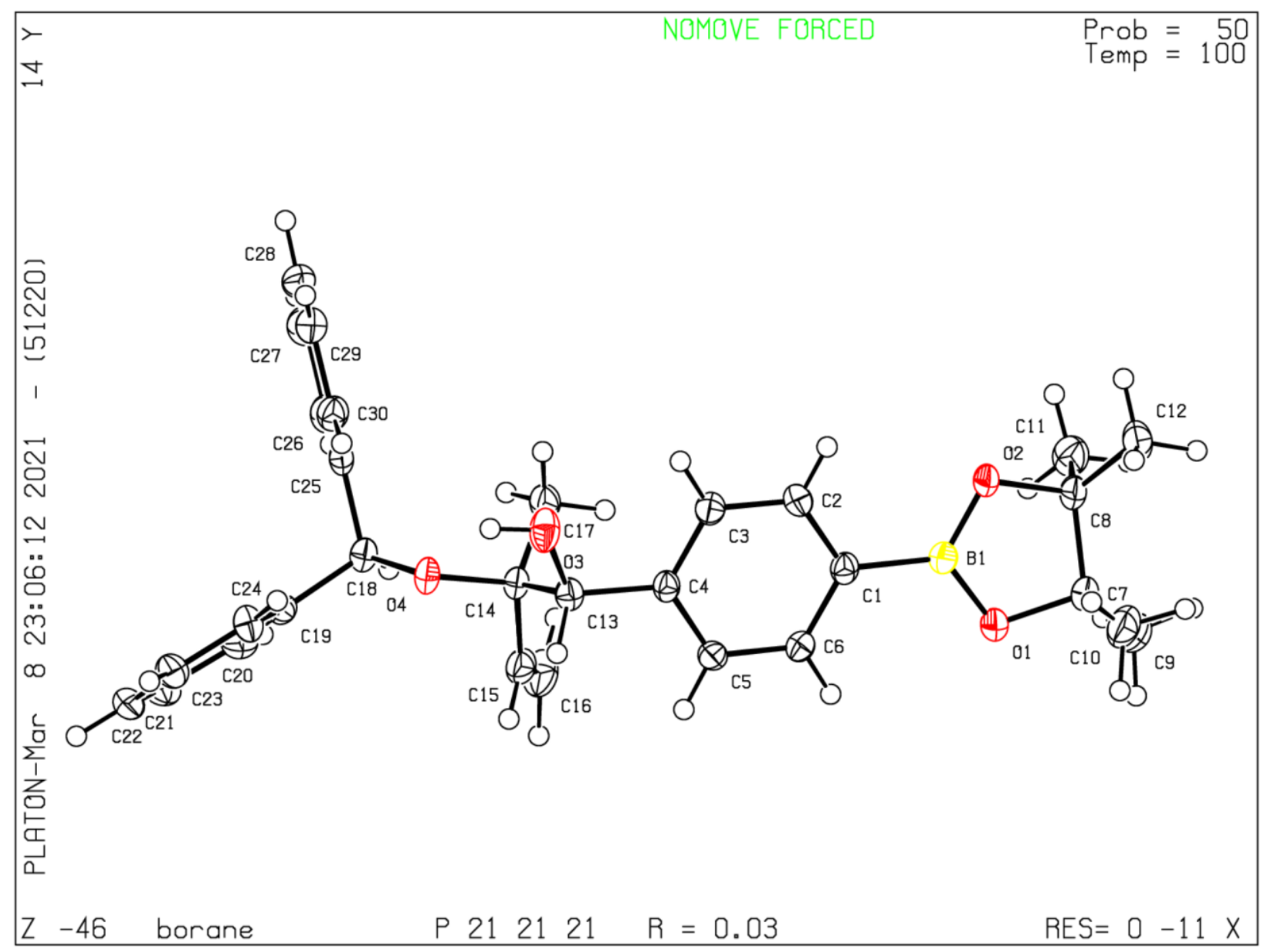




\section{Single Crystal Diffraction Data for Coupling Product 4j}

Empirical formula

Formula weight

Temperature

Wavelength

Crystal system

Space group

Unit cell dimensions

Volume

Z

Density (calculated)

Absorption coefficient

$\mathrm{F}(000)$

Crystal size

Theta range for data collection

Index ranges

Reflections collected

Independent reflections

Completeness to theta $=25.242^{\circ}$

Absorption correction

Max. and min. transmission

Refinement method

Data / restraints / parameters

Goodness-of-fit on $\mathrm{F}^{2}$

Final $\mathrm{R}$ indices [I $>2 \operatorname{sigma}(\mathrm{I})]$

$\mathrm{R}$ indices (all data)

Absolute structure parameter

Extinction coefficient

Largest diff. peak and hole
$\mathrm{C} 22 \mathrm{H} 22 \mathrm{O} 2 \mathrm{~S}$

350.45

$100.15 \mathrm{~K}$

$0.71073 \AA$

orthorhombic

P 212121

$\mathrm{a}=8.2177(8) \AA$

$\alpha=90^{\circ}$.

$\mathrm{b}=12.9880(12) \AA$

$\beta=90^{\circ}$.

$\mathrm{c}=17.0188(17) \AA$

$\gamma=90^{\circ}$.
4

$1.282 \mathrm{Mg} / \mathrm{m}^{3}$

$0.190 \mathrm{~mm}^{-1}$

744

$0.47 \times 0.32 \times 0.28 \mathrm{~mm}^{3}$

1.972 to $30.632^{\circ}$.

$-11<=\mathrm{h}<=11,-18<=\mathrm{k}<=18,-24<=1<=23$

47337

$5572[\mathrm{R}(\mathrm{int})=0.0464]$

$100.0 \%$

Numerical

1.0000 and 0.8456

Full-matrix least-squares on $\mathrm{F}^{2}$

$5572 / 0 / 240$

1.149

$\mathrm{R} 1=0.0324, \mathrm{wR} 2=0.0860$

$\mathrm{R} 1=0.0353, \mathrm{wR} 2=0.0870$

$0.00(7)$

$\mathrm{n} / \mathrm{a}$

0.379 and -0.297 e. $\AA^{-3}$ 
Figure 2. View of $\mathbf{4 j}$ showing the atom labeling scheme. Displacement ellipsoids are scaled to the $50 \%$ probability level.

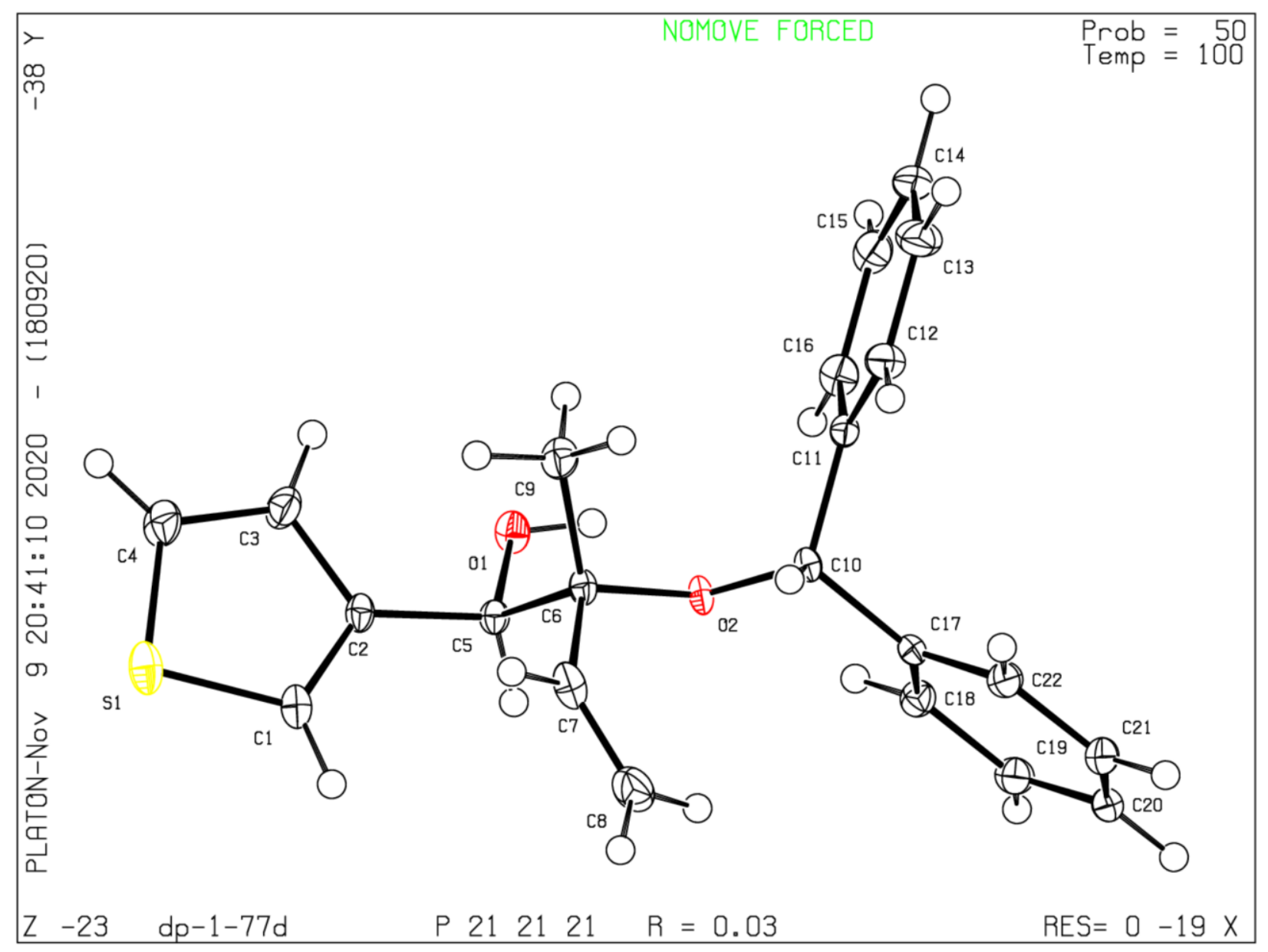




\section{Single Crystal Diffraction Data for Coupling Product 4hh}

Empirical formula

Formula weight

Temperature

Wavelength

Crystal system

Space group

Unit cell dimensions

Volume

Z

Density (calculated)

Absorption coefficient

$\mathrm{F}(000)$

Crystal size

Theta range for data collection

Index ranges

Reflections collected

Independent reflections

Completeness to theta $=67.684^{\circ}$

Absorption correction

Max. and min. transmission

Refinement method

Data / restraints / parameters

Goodness-of-fit on $\mathrm{F}^{2}$

Final R indices [I $>2 \operatorname{sigma}(\mathrm{I})]$

$\mathrm{R}$ indices (all data)

Absolute structure parameter

Extinction coefficient

Largest diff. peak and hole
C27 H34 O3

406.54

100.03(13) K

$1.54184 \AA$

orthorhombic

P 212121

$\mathrm{a}=6.10361(7) \AA$

$\alpha=90^{\circ}$.

$\mathrm{b}=14.89692(15) \AA$

$\beta=90^{\circ}$.

$\mathrm{c}=25.3054(2) \AA$

$\gamma=90^{\circ}$.

$2300.90(4) \AA^{3}$

4

$1.174 \mathrm{Mg} / \mathrm{m}^{3}$

$0.584 \mathrm{~mm}^{-1}$

880

$0.266 \times 0.06 \times 0.033 \mathrm{~mm}^{3}$

3.443 to $73.371^{\circ}$.

$-6<=\mathrm{h}<=7,-17<=\mathrm{k}<=18,-30<=\mathrm{l}<=31$

15182

$4547[\mathrm{R}(\mathrm{int})=0.0201]$

$100.0 \%$

Gaussian and multi-scan

1.000 and 0.806

Full-matrix least-squares on $\mathrm{F}^{2}$

4547 / 3 / 292

1.035

$\mathrm{R} 1=0.0273, \mathrm{wR} 2=0.0679$

$\mathrm{R} 1=0.0290, \mathrm{wR} 2=0.0690$

$0.04(5)$

$\mathrm{n} / \mathrm{a}$

0.185 and -0.150 e. $\AA^{-3}$ 
Figure 3. View of $4 \mathrm{hh}$ showing the atom labeling scheme. Displacement ellipsoids are scaled to the $50 \%$ probability level.

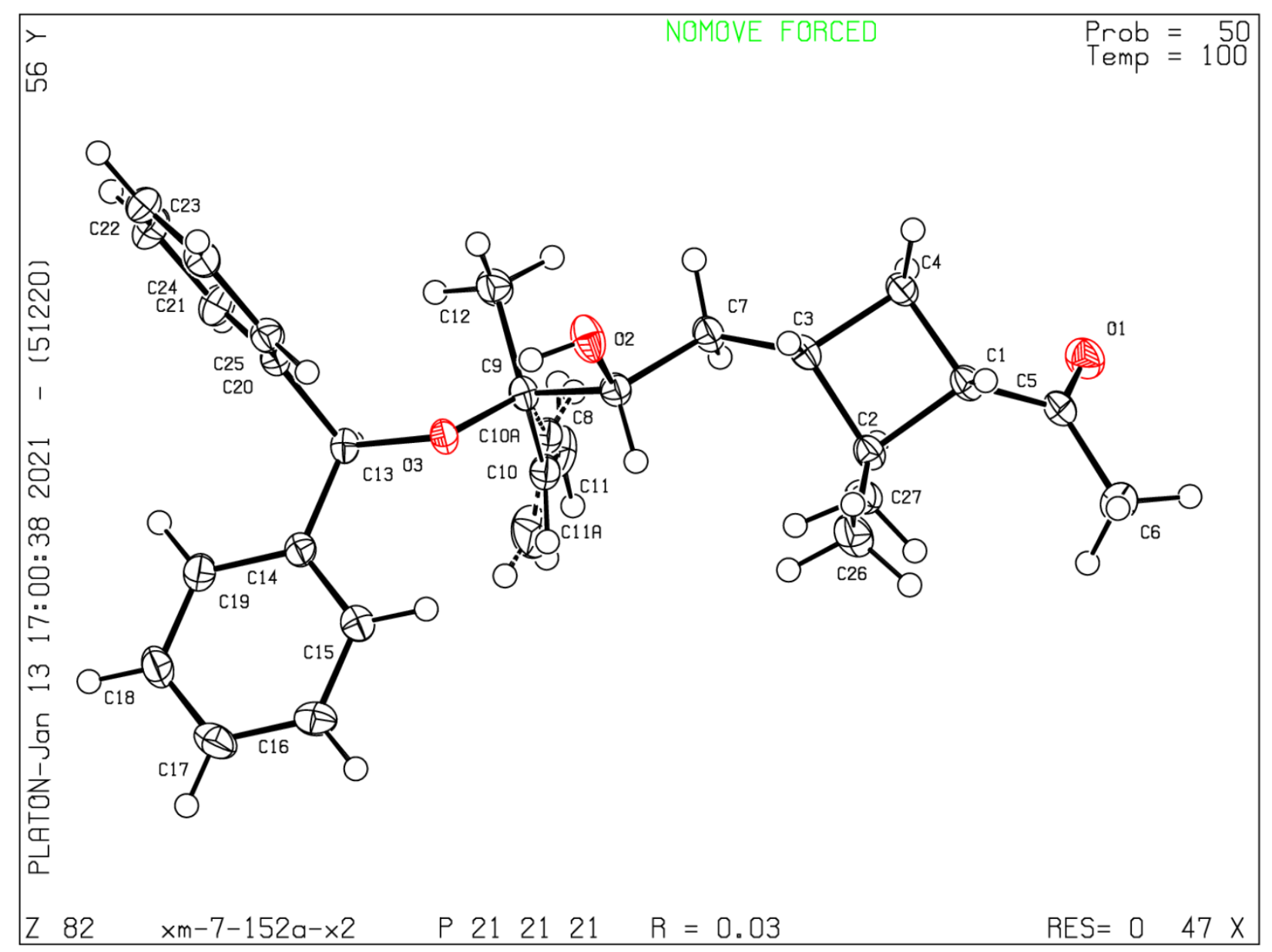




\section{$\underline{\text { References }}$}

(1) Trost, B. M.; Xie, J. J. Am. Chem. Soc. 2006, 128, 6044-6045.

(2) Jeffery, T. J. Chem. Soc., Chem. Commun. 1984, 1287-1289.

(3) Colbon, P.; Ruan, J. W.; Purdie, M.; Mulholland, K.; Xiao, J. L. Org. Lett. 2011, 13, 5456-5459.

(4) Sawant, P.; Maier, M. E. Tetrahydron, 2010, 51, 9738-9744.

(5) Pabbaraja, S.; Satyanarayana, K.; Ganganna, B.; Yadav, J. S. J. Org. Chem. 2011, 76, 19221925. 